\title{
Receptive field organization of motion computation in the fly: a study of cell types and their variability
}

\author{
Dissertation for the award of the degree \\ Doctor of Philosophy (PhD) \\ of the Georg-August-Universität Göttingen \\ within the doctoral program Neurosciences \\ of the Georg-August University School of Science (GAUSS) \\ submitted by \\ Luis Giordano \\ Ramos Traslosheros Lopez \\ from Toluca, Mexico
}

Göttingen 2019 


\section{Thesis Committee and Examination Board members}

First referee

Prof. Dr. Marion Silies

Institute of Developmental Biology and Neurobiology (iDN), Johannes Gutenberg

University Mainz

Second referee

Prof. Dr. Tim Gollisch

Sensory Processing in the Retina, Department of Ophthalmology, University

Medical Center Göttingen

Third referee

Prof. Dr. Fred Wolf

Theoretical Neurophysics, Department of Nonlinear Dynamics, Max Planck Institute for Dynamics and Self-Organization, Göttingen

\section{Further members of the Examination Board}

Prof. Dr. Tobias Moser

Institute for Auditory Neuroscience, University Medical Center Göttingen

Prof. Dr. André Fiala Dept. of Molecular Neurobiology of Behaviour, Schwann-Schleiden Research

Centre, University of Göttingen

Dr. Jan Clemens

Neural Computation and Behavior group, European Neuroscience Institute -

Göttingen 


\section{Contents}

Contents

List of Figures

List of Tables vii

1 Introduction $\quad 1$

1.1 Cell types . . . . . . . . . . . . . . . 2

1.2 Cell types in the Drosophila visual system . . . . . . . . . . . 9

1.3 Motion vision models . . . . . . . . . . . . . . . 11

1.4 Motion vision pathways . . . . . . . . . . . . . . 12

1.5 Thesis objectives . . . . . . . . . . . . 16

2 Functional variability within a cell type 19

2.1 Variability in the main input to OFF-DS neurons . . . . . . 20

2.2 Simultaneous receptive field measurement of two neuron

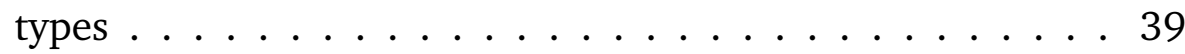

2.3 Receptive fields of main OFF-pathway interneurons . . . . 43

3 Receptive field mechanisms 49

3.1 Sketching the presynaptic circuitry of Tm9 neurons . . . . . 49

3.2 Cell type contributions to the receptive field of Tm9 . . . . . 60

3.3 Role of gap junctions in $\mathrm{Tm} 9 \ldots . \ldots 77$

4 Role of wide field neurons in motion computation 83

4.1 Receptive fields of distal medulla neurons . . . . . . . . 83

4.2 Wide-field inputs tune responses of direction-selective neurons 86

4.3 Response properties of T5 dendrites and associated Tm9 axons 99

5 Discussion 103 
5.1 Cell types with variable functional properties . . . . . . . . . 103

5.2 Why variability? . . . . . . . . . . . . . . . . . . 112

5.3 What we learned from motion detection . . . . . . . . . 114

5.4 Thoughts on research methodology . . . . . . . . . . . 115

5.5 Summary . . . . . . . . . . . . . 116

6 Materials and methods 119

6.1 Fly husbandry . . . . . . . . . . . . . . . . . . . . . . 119

6.2 Fly genotypes . . . . . . . . . . . . . . . . . . . . . . 119

6.3 Fly immunostainings . . . . . . . . . . . . . . . . . . 122

6.4 Fly in vivo two-photon calcium imaging . . . . . . . . . . . 122

6.5 Optogenetic input mapping . . . . . . . . . . . . 123

6.6 Data processing . . . . . . . . . . . . . . . . . . . . 124

6.7 ON-OFF full-field flashes analysis . . . . . . . . . . . . 126

6.8 Receptive field analysis . . . . . . . . . . . . . . . . . . 127

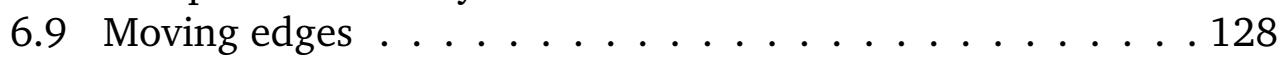

$\begin{array}{lr}\text { Bibliography } & 129\end{array}$

\section{List of Figures}

2.1 Tm9 responds preferentially to OFF full-field flashes. . . . . . . 21

2.2 PCA of Tm9 response to ON-OFF full-field flashes . . . . . . 23

2.3 First principal component of ON-OFF responses relates to the polarity . . . . . . . . . . . . . . . . 24

2.4 K-means clustering of Tm9 ON-OFF full-field flashes responses. 25

$2.5 \mathrm{Tm} 9 \mathrm{ON}-\mathrm{OFF}$ full-field flashes cluster properties . . . . . . . 27

2.6 Receptive fields of $\mathrm{Tm} 9$ neurons for flashing bars . . . . . . 28

2.7 Clustering of Tm9 ON-OFF full-field flashes responses is reproducible. . . . . . . . . . . . . . . . . 32

$2.8 \quad \mathrm{Tm} 9$ linear filters to full-field noise . . . . . . . . . . . . . . 33

2.9 Tm9 spatiotemporal receptive fields . . . . . . . . . . 35

$2.10 \mathrm{Tm} 9$ linear temporal filters . . . . . . . . . . . . . . 36 
2.11 Tm9 correlation of linear temporal filters across stimuli . . . . . 37

2.12 Noise receptive fields are larger than OFF and smaller than ON receptive fields . . . . . . . . . . . . . 38

2.13 ON-OFF full-field flashes responses of Tm9 and Tm4 measured simultaneously ..................... . 41

2.14 Receptive fields of Tm9 and Tm4 measured simultaneously . . . 42

2.15 Receptive field widths of Tm9 and Tm4 measured simultaneously 42

2.16 Responses of OFF-pathway neurons to ON-OFF full-field flashes 45

2.17 Receptive fields of the main inputs to the OFF pathway . . . . . 47

3.1 Input synapse distribution of the major OFF motion pathway interneurons ..................... 51

3.2 Mapping synapses from wide field neurons to Tm9 . . . . . . . 52

3.3 Dm4 and Dm12 pre- and postsynaptic sites distributions . . . . 54

3.4 Dm4 presynaptic site distribution with different markers . . . . 55

3.5 Cell types used for optogenetic mapping of Tm9 inputs . . . . . 57

3.6 Optogenetic stimulation at different light powers and durations 58

3.7 Functional connectivity mapping of Tm9 inputs . . . . . . . . 59

3.8 Responses to ON-OFF full-field flashes of Tm9 neurons without input from single lamina cell types. . . . . . . . . . . . . . . 61

3.9 Properties of response to ON-OFF full-field flashes of Tm9 neurons without input from single lamina cell types. . . . . . . . . 62

3.10 Receptive fields of Tm9 without input from lamina neurons L1, L2, or L3 . . . . . . . . . . . . . . . . . . . 66

3.11 Receptive field amplitude of Tm9 neurons upon blocking lamina neuron types L1, L2, or L3 . . . . . . . . . . . . . . 67

3.12 Receptive fields of Tm9 with single neuron type input silenced. Lamina neurons L1, L2 and L3 . . . . . . . . . . . . . 68

3.13 Responses to ON-OFF full-field flashes of Tm9 neurons without input from single wide-field cell types. . . . . . . . . . . . 69

3.14 Properties of response to ON-OFF full-field flashes of Tm9 neurons without input from single wide-field cell types. . . . . . . 70

3.15 Receptive fields of Tm9 without input from distal medulla neurons Dm4, Dm9, Dm12, or Dm20 . . . . . . . . . . . 74

3.16 Receptive field amplitude of Tm9 without wide-field inputs. . . 75

3.17 Receptive field width of Tm9 without input from distal medulla neurons Dm4, Dm9, Dm12, or Dm20 . . . . . . . . . 76

3.18 Responses to ON-OFF full-field flashes of Tm9 neurons without shakB-mediated gap junctions. . . . . . . . . . 78

3.19 Properties of response to ON-OFF full-field flashes of Tm9 neurons without shakB-mediated gap junctions. . . . . . . . 79 
3.20 Receptive fields of Tm9 without shakB-mediated gap junctions. 80

3.21 Receptive field amplitude and size of Tm9 without shakBmediated gap junctions. . . . . . . . . . . . . . . 81

4.1 Dm4 and Dm12 responses to ON-OFF full-field flashes . . . . 84

4.2 Dm4 and Dm12 example tuning curves to vertical ON and OFF

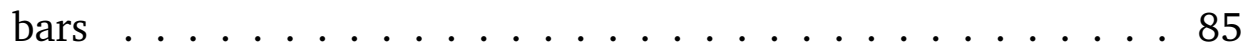

$4.3 \mathrm{~T} 4$ and T5 response maps and ROI selection $\ldots \ldots \ldots$. . . 87

4.4 T4 and T5 contrast selectivity to moving edges under block of wide-field and $\mathrm{Tm} 9$ inputs . . . . . . . . . . . . . 88

4.5 T4 and T5 responses to moving edges under block of wide-field and $\operatorname{Tm} 9$ inputs . . . . . . . . . . . . . . . 90

4.6 T4 and T5 response amplitude to moving edges under block of wide-field and $\mathrm{Tm} 9$ inputs . . . . . . . . . . . . . . . . 91

4.7 T4 and T5 direction selectivity and preferred directions to moving edges under block of wide-field and Tm9 inputs . . . . . . . 93

4.8 Distribution of preferred directions for $\mathrm{T} 4$ and $\mathrm{T} 5$ responses to moving edges under block of wide-field and Tm9 inputs . . . . 94

4.9 Correlation of T4 and T5 preferred directions at the same point in space under block of wide-field and Tm9 inputs . . . . . . . 95

4.10 Correlation of $\mathrm{T} 4$ and $\mathrm{T} 5$ direction selectivity index at the same point in space under block of wide-field and Tm9 inputs . . . . 96

4.11 T4 and T5 direction selectivity to moving edges under block of wide-field and $\mathrm{Tm} 9$ inputs . . . . . . . . . . . . . . . 97

4.12 T4 and T5 direction selectivity over cardinal directions under block of wide-field and Tm9 inputs . . . . . . . . . . . . . 98

4.13 OFF receptive field properties of overlapping $\mathrm{T} 5$ and $\mathrm{Tm} 9$ neurons 100

4.14 Spatiotemporal tuning maps of T5 dendrites and overlapping Tm9 axons are correlated . . . . . . . . . . . . . . 102 


\section{List of Tables}

2.1 Statistics for $\mathrm{Tm} 9$ receptive field size across stimuli. . . . . . . . 40

3.1 Statistics for Tm9 ON-OFF full-field flashes response properties under block of lamina neurons. . . . . . . . . . . . . 60

3.2 Statistics for $\mathrm{Tm} 9$ receptive field widths under block of lamina neurons. . . . . . . . . . . . . . . . 65

3.3 Statistics for $\mathrm{Tm} 9 \mathrm{ON}-\mathrm{OFF}$ full-field flashes response properties under block of wide field neurons. . . . . . . . . . . . 71

3.4 Statistics for $\mathrm{Tm} 9$ receptive field widths under block of widefield neurons. . . . . . . . . . . . . . . 72

3.5 Statistics for Tm9 ON-OFF full-field flashes response properties under gap junction block. . . . . . . . . . . . . 77

3.6 Statistics for Tm9 receptive field size under gap junction block. 82

4.1 Statistics for T4 and T5 contrast selectivity to moving edges under block of wide-field and Tm9 inputs . . . . . . . . . 89

4.2 Statistics for T4 and T5 response amplitude to moving edges under block of wide-field and Tm9 inputs . . . . . . . . . . . 89

4.3 Statistics for T4 and T5 direction selectivity to moving edges under block of wide-field and Tm9 inputs . . . . . . . . . 97

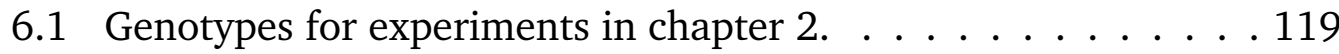

6.2 Genotypes for experiments in section 3.1. . . . . . . . . . . . 120

6.3 Genotypes for experiments in sections 3.2 and 3.3. . . . . . . . 121

6.4 Genotypes for experiments in chapter $4 \ldots 121$ 



\begin{abstract}
It is believed that knowing all cell types making up a brain will lead to its understanding. Consequently, current efforts focus on classifying cell types according to their anatomical, genetic and physiological properties. Research in Drosophila melanogaster has greatly advanced cell type classifications, using transcriptomics, connectomics and genetic tools for neural circuit dissection. In particular, the fly eye, or optic lobe, has been extensively researched in an attempt to understand motion computation. Detection of motion is crucial for the survival of many species. Furthermore, motion computation is close to being mapped to its algorithmic neuronal substrate. In this thesis, we map the functional circuit organization of the fly visual system at the level of cell types. We focus on Tm9, the main synaptic input to T5 neurons, the first direction-selective neurons in the fly visual system, which detect moving dark edges. Tm9 like most neurons in Drosophila melanogaster has a stereotyped anatomy, that distributes regularly over the 800 columns of the fly eye and can be specifically targeted with genetic lines. Here, using in vivo two-photon calcium imaging we show $\mathrm{Tm} 9$ has variable receptive field properties that contrast with its homogeneous anatomy and genetics. In particular, Tm9 can display both narrow and wide receptive fields. We demonstrate this variability is not common to other fly neurons using simultaneous dual imaging, and find that wide-field responses are mediated by ON signals. Using anatomical and optogenetic circuit mapping, we identify Dm4, Dm12, and Dm20 as novel wide-field (variable) inputs to Tm9, and combine genetic silencing with in vivo imaging to show each of them mediates Tm9 response properties. In particular, Dm12 and Dm20 sharpen Tm9 ON receptive fields. The influence of Dm4, Dm12, Dm20, and Tm9 extends to downstream computations, genetic silencing reveals they are all required for proper directional tuning of T4 and T5 neurons. We further establish a computational role of functional variability in Tm9 by showing that Tm9 axons' spatiotemporal properties correlate with the ones from postsynaptic T5 dendrites. Taken together our results unravel the existence of variability within classical cell types, its functional relevance in motion detection, and show newly characterized wide-field neurons in the fly brain are required for motion computation. We postulate flexible synaptic connectivity as a mechanism to regulate functional heterogeneity in morphologicallyand genetically-defined cell types.
\end{abstract}





\section{$-1-$ \\ Introduction}

This thesis aims to analyze the concept of neural identity and its precision in the context of a behaviorally relevant computation. This study focuses on neuron types in the Drosophila visual system required for motion detection.

\section{What is the goal of neuroscience? Theory of the brain?}

The goal of science is to reach a level in which the theories can explain known and predict unknown phenomena. In neuroscience, we study the brain, one of the most complex systems. Even more complex is the collective behavior arising from the interaction of individuals with brains. We assume the brain is the substrate for the mind. And thus, the brain allows us to sense, perceive, act, form memories, and retrieve them. This knowledge goes back at least to 400 B.C.E. when Hippocrates wrote his treatise "On the Sacred Disease" 1 :

Men ought to know that from nothing else but the brain come joys, delights, laughter and sports, and sorrows, griefs, despondency, and lamentations. And by this, in an especial manner, we acquire wisdom and knowledge, and see and hear, and know what are foul and what are fair, what are bad and what are good, what are sweet, and what unsavory; some we discriminate by habit, and some we perceive by their utility.

Through neuroscience, we face the opportunity to dive into the very essence of our thinking, to figure out how the brain works.

\footnotetext{
2019)

${ }^{1}$ See translation in (The Internet Classics Archive — On the Sacred Disease by Hippocrates
} 


\section{$1.1 \quad$ Cell types}

Analyzing the brain a neuron at a time To understand the brain, one needs to understand its composition. One milestone in this direction was the emergence of the neuron doctrine. This doctrine, pioneered by Santiago Ramón y Cajal, stated the brain consisted of individual discrete units called neurons. This advancement was enabled by a silver nitrate staining technique developed by Camillo Golgi (the "reazione nera"). Thereby, brain tissue was stained to be visualized under a microscope. Ramón y Cajal's observations led to postulating the existence of neurons. He also pioneered the description of the nervous system organization in several organisms, as compiled in his many engravings. Neuronal types emerged from reoccurring shapes of neurons and their organization into circuits. Thus, studying the myriad neurons of the brain can be easier if we group them into fewer discrete types. Through classification we can describe a system in a hierarchical order of detail. This way we can focus on fewer items at a time and overcome the limits of our cognition. Therefore, cell type classifications, of neuronal and non-neuronal cells, are useful as an inventory of brain composition.

The importance of classifications of cells, has led to an avalanche of information that cannot be summarized in this thesis introduction, for different perspectives some reviews are suggested (Masland, 2004; Seung and Sümbül, 2014; Poulin et al., 2016; Arendt et al., 2016; Zeng and Sanes, 2017). This interest was fueled by major funding initiatives, e.g., classifying cell types is the first aim of the BRAIN initiative ("BRAIN 2025: A Scientific Vision" n.d.). Consequently, even entire institutes dedicate themselves to classify all cell types in human and mouse brains (cortices) (Ecker et al., 2017).

Historically, the first neuron characterizations were purely anatomical. Later advances for measuring the electric properties of neurons allowed to classify neurons into electrophysiological neuronal types. With advances in genetics, neurons could also be classified on the basis of their expressed genes, creating genetic cell types with ever larger specificity. With the ability to genetically label cell types and trace their projections, connectivity was a further addition to the neuron classification schemes. Serial electron microscopy paved the way for neuron reconstruction and synapse annotation to map the connectome, as a further source of connectivity classification for neuronal cell types.

Nowadays, high-throughput experiments attempt to sample freely from bias the anatomical, genetic, and physiological properties of neurons in brain regions with some known functions (Zeisel et al., 2015; Gouwens et 
al., 2019) and others with functions yet to unravel (Namboodiri, RodriguezRomaguera, and Stuber, 2016; Pandey et al., 2018). Next, we expand on these aspects.

\section{Anatomy}

First, we discuss the role of anatomical methods in the classification of neuronal types. Golgi stain labels tissue randomly and sparsely, and can isolate single neurons. This method and its iterations are particularly useful for exploring neural organization in species with limited genetic access like camels (Zaqout et al., 2012), lizards (Srivastava, Maurya, and Chand, 2009), and humans (Al-Hussain Bani Hani, Al-Haidari, and Saboba, 2007).

Besides Golgi method, neurons were also reconstructed from electron microscopy images. Both methods are low-throughput. An attempt to scale up the number of neurons sampled in a tissue came about with connectomics. Connectomics aims to reconstruct of all neurons and their connections in a brain area, or ideally the whole brain or even organism. The typical method for connectomics is the reconstruction of a volume scanned with electron microscopy (Denk and Horstmann, 2004; Briggman and Bock, 2012). This approach has shed light on circuit computations such as direction selectivity in several species (Briggman, Helmstaedter, and Denk, 2011; Takemura, Bharioke, et al., 2013), and contributed with discovery of new cell types (Helmstaedter et al., 2013). This, in principle, enables sampling without bias all neurons in a brain. For example, a whole Drosophila melanogaster brain was imaged using electron microscopy and is available for tracing (Zheng et al., 2018).

The following methods illustrate the combination of multiple features in the efforts to reach comprehensive cell type classifications. To link morphology to electrophysiology, single cells are also labeled and traced after recording their electrical activity using patch-pipettes. This method is currently used to push the classification of cortical neurons, which could as well split into the order of 1000 neuron types (Masland, 2004). For example, morphological and electrophysiological data were used to model a cortical column of the somatosensory cortex of the rat (Markram et al., 2015). And more recently, visual cortical neurons were classified based on joint electrical and morphological properties (Gouwens et al., 2019).

Genetic methods also contributed to the anatomical studies of neurons. Neurons can be visualized via expression of fluorescent proteins driven by some genetic line (Feng et al., 2000) or viral vectors (Moriyoshi et al., 1996). Viral methods are also useful to label neurons retrogradely or 
anterogradely, important in mapping projections and synaptic partners. The labeling coverage of these methods could range from sparse to dense. To ensure labeling single neurons, stochastic expression methods were developed for a single fluorescent protein (Struhl and Basler, 1993; Wong, Wang, and Axel, 2002). To increase the sampling coverage, the stochastic labeling method was extended to multiple spectrally different fluorescent proteins (Livet et al., 2007; Hadjieconomou et al., 2011; Hampel et al., 2011; Cai et al., 2013; Nern, Pfeiffer, and Rubin, 2015). This increased the number of distinct colors to label neurons. And even allowed to identify novel neuron types (Nern, Pfeiffer, and Rubin, 2015).

Another assumption is that neurons of a same type would have similar connectivity, so neurons of the same type connect to the same neuron types in similar proportions (Seung and Sümbül, 2014). A prime example of connectivity classification is work on the nematode Caenorhabditis elegans, which 302 neurons were classified into 118 classes based on morphology and connectivity (J.G. White et al., 1986; Hobert, Glenwinkel, and J. White, 2016). Clearly, anatomical methods have pushed forward classification since the beginning of the modern age of neuroscience, and even more aided by advances in molecular biology and imaging techniques.

\section{Physiology}

Anatomical methods show the static properties of neurons. However, neurons perform their function dynamically, shaping the information that flows through them. In turn, neurons properties can change depending on the dynamics of its activity. The function of a neuron is usually measured as its activity under certain context, e.g., external stimuli and internal states. A functional definition of a cell type requires neurons of a single type to have the same function.

Classification of neuronal function was traditionally done recording electrical activity of single or couple of neurons at a time. Classifications depended on the feature chosen by the experimenters, in some cases reaching a convention (The Petilla Interneuron Nomenclature Group (PING), 2008). Electrophysiological recordings offer high spatial and temporal resolution to neuronal properties. Given the low throughput of such recordings, it is useful to follow them by labeling and tracing the neuron, or sequencing its molecular properties (Markram et al., 2015; Cadwell et al., 2016; Gouwens et al., 2019).

Conversely, optical methods offer access to larger number of neurons simultaneously. Recording of neuronal populations at cellular resolution relies on fluorescent indicators of voltage and calcium. Voltage recordings 
reflect closer neural activity than calcium. But because of the lower sensitivity of voltage indicators, calcium indicators are often used for large-scale recordings (T.-W. Chen et al., 2013; W. Yang and Yuste, 2017). Light scattering hinders the study of deep regions in larger brains, but whole brain recordings at cellular resolution are possible in smaller translucent animals (Ahrens et al., 2013; Nguyen et al., 2016).

To split neurons into functional cell types, the activity of neurons is recorded in response to a set of stimuli sampling a wide array of response properties. Then, quantitative data analysis is performed as objectively as possible to get an unbiased classification using statistical techniques. With this approach, populations of retinal bipolar and ganglion neurons in the mouse were classified into functional types based on responses to an array of visual stimuli (Franke et al., 2017; Baden et al., 2016). Further improvements in microscopy and activity sensors will scale up the number of neurons that can be recorded, and allow to reliably record voltage and other modalities of neural activity. Classifications based on functional imaging are yet to be applied in many brain regions and species.

\section{Genetics}

We have discussed about the morphology and function of neurons. These properties largely boil down to the genes expressed in the neuron. Therefore, one can expect that the genetic identity of neurons can provide another mean for cell type classification. Reliably identifying cell types based on genetic properties allows for systematic targeting of the same neurons in different animals. Thus, genetic access to single cell types is useful to study their anatomical and functional properties.

Earlier tools to access cell types, relied on enhancer/promoter elements that produced a desired expression pattern. Driver lines were created to express transgenes using an endogenous gene specific to a cell type. Another method to express transgenes is enhancer traps, insertions of transgenes into random locations in the genome (Bellen et al., 1989; Brand and Perrimon, 1993). This method provided some useful lines but usually not at a cell type level.

Greater specificity was achieved using binary systems. In binary systems, a cell-type specific promoter drives an effector transgene, thus only in cells where both parts come together the transgene is expressed. A successful binary system is the Gal4/UAS system in Drosophila (Fischer et al., 1988; Brand and Perrimon, 1993). Here, the promoter drives expression of the transcription factor Gal4, and the effector transgene is under control of the UAS (upstream activation sequence). Thus, the effector 
transgene is expressed only when Gal4 comes together with UAS. Usually, two transgenic animals carry each part of the binary expression system to allow for different combinations of transgenes and cell types. For species with no transgenic methods the transgenes are inserted using viral vectors (El-Shamayleh, Ni, and Horwitz, 2016). These methods allowed defining some cell types by the expression of fluorescent proteins in selected neurons as discussed above (p. 4). Thus, lines were cell type specific when the resulting expression patterns labeled a subset of neurons with similar anatomical properties. So with this approach, genetic and anatomical definitions of cell types are intermingled. For further information on genetic approaches to neuroscience see (Luo, Callaway, and Svoboda, 2008; Luo, Callaway, and Svoboda, 2018).

Previous methods were labor intensive, and cell-type definitions relied on the visualization of neuron morphologies. Current methods allow for high-throughput unbiased characterization of genetic cell types based on single-cell transcriptomes. The transcriptome is the collection of all expressed mRNAs. In this approach single cells are sequenced and classified into types based on their gene expression patterns. The classification resorts to statistical methods similarly to the ones used for anatomical or functional properties. For a review see (Poulin et al., 2016; Zeng and Sanes, 2017). In particular, retinal bipolar cells were sequenced and classified into clusters that recapitulate morphological and functional types (Shekhar et al., 2016; Helmstaedter et al., 2013; Franke et al., 2017). In the cortex, different studies have shown classifications that seem to share grouping of major known classes of excitatory and inhibitory neurons, and further clusters that may represent new types (Cadwell et al., 2016; Tasic, Menon, et al., 2016; Tasic, Yao, et al., 2018). In C. elegans transcriptomic classifications largely match the anatomical one (Hobert, Glenwinkel, and J. White, 2016). Transcriptomic studies can also identify specific markers to target a specific cell type, and further contribute to the study of its anatomical and functional properties.

The relation between the classification of neuronal types using anatomy, physiology or genetics is not trivial. In the retinal bipolar cells multiple independent classifications of anatomy, physiology and genetics match. But in the case of cortical neurons grouping by physiology or anatomy alone yields different results than using both properties simultaneously (Gouwens et al., 2019). Also the factors are interrelated: genetics defines electrical and morphological properties, and connectivity, but activity in turn alters gene expression and connectivity. This interaction makes a unified cell-type definition non-trivial. Therefore, neurons should be classified comprehensively based on joint anatomical, functional, and 
genetic properties.

\section{Data analysis for cell type classifications}

In all previous approaches mentioned, classification involved techniques for visualization, dimensionality reduction, and clustering of high-dimensional data. Here, we just want to draw attention to the role of existing algorithms in defining a cell type, as there is no all-purpose method to cluster data in a completely unbiased and unsupervised way. Usually analysis commences with some visualization, to project the data into two or three dimensions. Consider the example of a transcriptomic sequencing: $N$ neurons each of them expressing some combination of $n$ possible genes. This data set lies in an $n$ dimensional space, where each dimension corresponds to the expression level of one of the genes. In this space, each neuron will correspond to a point, an $n$-dimensional vector, where the $i$-th component is the expression level of the gene $i$. If the sequencing data provides information about hundreds or thousands of genes, we can see how unintuitive this space will be. Using dimensionality-reduction techniques (typically PCA, NMF, t-SNE, WGCNA) will allow projection of the data into a new set of coordinates, ordered by their contribution to represent the original high-dimensional data. Thus, we can visualize the data in two or three dimensions and try to find patterns or groups. This step is useful, as there is no optimal way of establishing the number of clusters an algorithm is expected to find. Even unsupervised algorithms depend on the choice of a parameter to optimize, e.g. distance, or similarity metric between data points, and also depend on a cutoff to define the final cluster arrangement, e.g., hierarchical clustering. The full data in the original form (or in a reduced number of dimensions if data size is too large) can be fed into a clustering algorithm (k-means, hierarchical clustering, mixture of Gaussians, etc.). The resulting clusters can be then visualized in the reduced space provided by the previous step. This approach of clustering and visualization can iterate until a satisfactory grouping is achieved. The satisfaction criteria are sometimes defined by the expected number of clusters based on previous studies of anatomical or functional types. Therefore, we need to keep in mind that even when we cluster data, the underlying structure can be a continuum of cell type properties. Also with larger number of neurons available new types can be discovered, previous types can be merged into one type, or a type can be split into new ones. 


\section{Using cell types to describe neural circuits and their computations}

Cell types are useful to understand neural computations and behavior. One can aim to describe the neural substrate of a computation with a canonical circuit of cell types. Conversely, one can identify cell types and study their function until they can be ascribed to a given computation or behavior. Either way requires defining cell types according to their function. But going from a computation to the neural substrate may avoid the pitfalls of an otherwise reductionist approach from neurons to computations/behavior (Krakauer et al., 2017). Thus, cell types should be studied under their computational and behavioral context.

To dig into the cell type contributions to the algorithmic implementation of a computation we can focus on organisms and computations with a large knowledge foundation. Many computations are conserved across species, however, the concrete implementation may differ. For example, various animals use the direction of motion to navigate the environment. Because of its behavioral relevance and experimental accessibility, the computation of motion has been actively researched since the 1950s, in both invertebrates (Hassenstein and Reichardt, 1956), and vertebrates (Barlow and Levick, 1965). And similar mechanisms with some crucial differences were proposed for different species (reviewed in (Ramos-Traslosheros, Henning, and Silies, 2018)). However, this mechanisms were algorithmic and lacked detailed implementations at the neuronal level. This ignited the search for the cell types which properties could fit into the algorithmic description.

With advances in genetics, Drosophila melanogaster became a prime candidate to look for the neuronal substrate of motion detection (Rister et al., 2007), eventually unraveling the first direction-selective neurons in the fly eye (Maisak et al., 2013). Prior to genetics, cell types were defined in Drosophila melanogaster based on anatomical studies (Fischbach and Dittrich, 1989). Further refinements in the genetic tools allowed greater specificity, targeting single anatomical cell types (Pfeiffer et al., 2010). Consequently, research increased on cell type physiological properties and their role in motion computation (Joesch et al., 2010; Clark et al., 2011; Silies et al., 2013; Behnia et al., 2014; Fisher, Leong, et al., 2015; Serbe et al., 2016; Arenz et al., 2017; Strother et al., 2017). Therefore, motion computation in the fly enables the study of single cell types within their computational context. 


\subsection{Cell types in the Drosophila visual system}

Drosophila melanogaster stands in a good place regarding cell type classifications thanks to recent advances, in particular, since the start of this thesis. The list of parts of the brain in the fly is closer to completion than in other species, with the exception of the nematode worm Caenorhabditis elegans which 302 neurons have been classified based on anatomy, connectivity, or genetics (Hobert, Glenwinkel, and J. White, 2016). As for Drosophila melanogaster a whole brain has been imaged using electron microscopy and is available for segmentation and mapping of neural circuits (Zheng et al., 2018). Particularly, at the level of the fly visual system, we have access to anatomically defined cell types, connectomes, transcriptomes, and behavioral and physiological studies with cell type specificity.

\section{Characterization of cell types in the optic lobe}

\section{Anatomical classification: Golgi studies, fluorescence labeling, and connectomics}

The fly visual system has attracted scientists since the beginning of modern neurobiology (Cajal, 1909; Ramon y Cajal and Sánchez, 1915). The first anatomical descriptions were done in larger fly species. Later on, Golgi studies revealed morphological cell types in the Drosophila melanogaster visual neuropiles, the optic lobe (Fischbach and Dittrich, 1989). And modern genetic labeling tools revealed even more neuron types (Nern, Pfeiffer, and Rubin, 2015). The fly visual system is so stereotypical that single neurons from different individuals can be ascribed to one type based purely on anatomy.

The stereotypical constitution of the fly brain makes anatomical cell types the foundation for genetic and physiological studies. Genetic lines to label cell types are defined by their expression pattern. To label a single cell type, the resulting expression pattern has to comprise a uniform distribution of cells matching the anatomical features of the cell type of interest. Once a specific genetic line for a cell type is available, one can study the genetic and physiological properties of the cell type. In particular, genetic lines for single cell types are ground truth for transcriptomic approaches to cell type classification, and for labeling neurons reconstructed from connectomics.

On the connectomics part, synaptic organization of the optic lobes was first restricted to the connections in the second neuropile, the lamina (Meinertzhagen and O'Neil, 1991). Further studies reached out to the 
next neuropile, the medulla (Takemura, Bharioke, et al., 2013; Shinomiya, Karuppudurai, et al., 2014; Takemura, Xu, et al., 2015), and finally to the lobula and lobula plate (Shinomiya, Huang, et al., 2019).

\section{Genetic classification: driver lines and transcriptomes}

Over the years, collections of thousands of genetic lines to target Drosophila melanogaster neurons have achieved higher specificity. From the first collections of promoter/enhancer pairs, e.g., the binary expression system GAL4/UAS (Brand and Perrimon, 1993; Jenett et al., 2012), to lines relying on genetic intersections to narrow down the expression pattern, e.g., splitGAL4 (Pfeiffer et al., 2010; Gohl et al., 2011; Tirian and Dickson, 2017; Dionne et al., 2018).

On the other hand, transcriptomic studies have sequenced optic lobes (Konstantinides et al., 2018) or brains (Davie et al., 2018) at the single cell level and classified neurons intro genetic cell types. But these studies usually cannot recover the number of cell types expected from anatomy (Davis et al., 2019). A recent study departs from specific genetic lines to obtain transcriptomes of single cell types (Davis et al., 2019). Thus, this approach bridges anatomical, genetic, and physiological studies based on the same genetic line.

\section{Functional classification: very rough, behavior driven, forward or backward screening}

Given the genetic accessibility of the fly, physiological studies often rely on genetic lines to express neural activity indicators. Unlike mammalian neurons, the soma of neurons in the fly lies far apart from the neurites, where synaptic transmission takes place. Because of this and the general disadvantages of small structures for electrophysiology, imaging of neuronal activity is the conventional choice to study neuronal processing (T.-W. Chen et al., 2013; H.H. Yang, St-Pierre, et al., 2016). Furthermore, large scale studies are precluded by the dense neuropile that hinders single cell segmentation from whole brain imaging. Thus, studies of neuronal function are as narrow or broad as the genetic line expression pattern. Therefore, most studies in the fly use specific genetic lines to image single cell types, an unparalleled advantage over other organisms. 


\subsection{Motion vision models}

Here, we give a brief account of the algorithmic descriptions of motion computation that fueled the search for the precise neural implementation of motion detection. For a more detailed review see (Ramos-Traslosheros, Henning, and Silies, 2018; H.H. Yang and Clandinin, 2018).

Motion in essence can be detected up to the spatial resolution of a given visual system. Motion at this scale is encoded by elementary motion detectors (EMD). This units respond selectively to one direction of motion, i.e., they are direction-selective (DS).

First mechanistic studies of motion detection date back to the 1950s, when using the Chlorophanus beetle Hassentein and Reichardt proposed a model for direction selectivity (Hassenstein and Reichardt, 1956). The model, nowadays known as Hassenstein-Reichardt Correlator (HRC), computes motion using two channels originating from two spatial points in visual space. The points are offset in space, and one channel is processed with a delay with respect to the other. The two channels are then nonlinearly amplified at the output stage, resulting in a spatiotemporal correlation, hence the name.

For example to encode left-to-right motion, the corresponding HRC has a delay in the left channel. When the left channel is activated by left-to-right motion, the signal is delayed, there is also an inherent motion delay, the time needed from the motion to traverse the spatial offset of the channels. Depending on the speed of motion, the signals will overlap at the output stage, and produce an amplified output signaling left-to-right motion. For right-to-left motion, the delay of motion adds to the processing delay, thus, signals arrive individually at the output stage and are not amplified and do not signal motion. Therefore, to generate direction selectivity the HRC amplifies signals in the preferred direction of motion (PD), not in the null direction (ND).

A similar two channel EMD model, the Barlow-Levick Model (BLM), originated from studies of direction-selective neurons in the rabbit retina (Barlow and Levick, 1965). The two channels are also spatially offset and processed with a temporal delay. In this case signals are combined in a suppressive way, via a NOT AND operation. The suppression originates from a NOT operation in the delayed arm. As long as a signal is present in the delayed channel, the outcome of the AND operation and the EMD model will be zero. For a EMD with left-to-right PD, the delayed suppressive channel will be in the right channel. The delay of movement plus processing delay allow signals in the PD to arrive before the suppressive signal, thus signaling motion. For motion in the ND, the delayed signal 
in the suppressive channel overlaps temporally with the excitatory signal from the other channel, nullifying the output signal. Therefore, to generate direction selectivity the BLM suppresses signals in the null direction of motion, not in the preferred direction.

The motion energy model was proposed to explain motion detection in humans (Adelson and Bergen, 1985). However, this model, under some conditions, is fully equivalent to the HRC (van Santen and Sperling, 1985).

The predictions of the HRC model were verified in flies and other organisms. Nevertheless, recent studies reveal the mechanism of direction selectivity is more complex than any of the discussed models.

\subsection{Motion vision pathways}

We discussed the resources available for studying cell types in the optic lobes, and classical models for motion computation. Now we describe the mapping of those cell types into the algorithmic implementation of motion detection.

\section{Anatomy of the fly eye}

The fly eye comprises the retina and the optic lobe. The optic lobe of the fly comprises four neuropiles: the lamina, the medulla, the lobula, and the lobula plate.

The fly eye has a lattice arrangement consisting of eye columns, roughly 800 of them. In the retina, each column is called an ommatidium. One ommatidium contains eight types of photoreceptors R1-R8. Photoreceptors R1-R6 express the broadband rhodopsin Rh1 in all ommatidia (Ostroy, M. Wilson, and Pak, 1974; O'Tousa et al., 1985; Zuker, Cowman, and Rubin, 1985), whereas R7 and R8 exist in two subtypes that define two types of ommatidia: pale and yellow. Pale ommatidia have R7 that express Rh3 sensitive to UV, and R8 that express Rh5 sensitive to blue. Yellow ommatidia have R7 with Rh4, also sensitive to UV but of longer wavelengths, and R8 with Rh6 that is sensitive to green (Zuker, Montell, et al., 1987; Montell et al., 1987; Salcedo et al., 1999). Pale and yellow ommatidia are stochastically distributed over the retina (Chou et al., 1999).

The R1-R6 neurons project to the lamina in a retinotopic fashion, so axons carrying information from the same region of space project to the same region of the lamina (Braitenberg, 1967; Horridge and Meinertzhagen, 1970). This retinotopic projection along with other cell types in the lamina form a cartridge. The lamina cartridge, together with the R7 and R8 
axons, then projects to the medulla, conserving the retinotopy (Horridge and Meinertzhagen, 1970).

From the twelve types of lamina neurons there is an expansion to the more than sixty types of the medulla. Most cell types are columnar and each cartridge has a copy of them, while other cell types span multiple columns and are less numerous than the number of cartridges (Fischbach and Dittrich, 1989). However, few lamina cell types are required for motion detection (Tuthill et al., 2013; Silies et al., 2013), and a fewer proportion in comparison in the medulla (Ammer et al., 2015; Fisher, Leong, et al., 2015; Serbe et al., 2016; Strother et al., 2017), reviewed in (Ramos-Traslosheros, Henning, and Silies, 2018; H.H. Yang and Clandinin, 2018). The first neurons to compute motion in the fly eye have their axons in the lobula plate, and their dendrites in either the medulla or the lobula. Next, we focus on the neurons with some role in motion detection.

\section{Anatomy of motion pathways}

The first neurons computing motion in the fly are the direction selective neurons T4 and T5 (Maisak et al., 2013). Both have their axons in the lobula plate, but T4 has dendrites in the proximal medulla, and T5 in the first layer of the lobula. The two neuron types are selective for stimuli of opposite polarity: T4 and T5 neurons are selective for bright, and dark moving edges, respectively (Maisak et al., 2013; Fisher, Silies, and Clandinin, 2015). Furthermore, T4 and T5 neurons exist in four subtypes, each type projects exclusively to one of the four layers of the lobula plate (Fischbach and Dittrich, 1989). Interestingly, each T4 and T5 subtype responds preferentially to one of the four cardinal directions (E. Buchner, S. Buchner, and Bülthoff, 1984; Maisak et al., 2013; Fisher, Silies, and Clandinin, 2015). T4 and T5 neurons are required for behavioral responses to motion (Maisak et al., 2013; Bahl et al., 2013; Serbe et al., 2016; Strother et al., 2017; Leonhardt, Meier, et al., 2017).

Now we describe the pathways leading to the direction selective neurons $\mathrm{T} 4$ and $\mathrm{T} 5$, the $\mathrm{ON}$ and the OFF motion pathways, respectively. Both pathways start in the retina, photoreceptors R1-R6 were already known to be required for motion vision (Heisenberg and E. Buchner, 1977; Yamaguchi et al., 2008), and R7 and R8 to have a minor contribution (Wardill et al., 2012). R1-R6 synapse to the lamina monopolar neurons L1, L2, and L3. Silencing the outputs of the lamina neurons in different combinations led to the observation of the ON and OFF motion pathways. The ON motion pathway starts with L1 neurons, while the OFF motion pathway starts with L2 and L3 neurons (Rister et al., 2007; Joesch et al., 2010; 
Clark et al., 2011; Silies et al., 2013). Lamina neurons project to the medulla, where the pathways diverge through several interneurons before converging back into T4 and T5, conserving the retinotopy throughout (Shinomiya, Huang, et al., 2019).

The following is extracted from recent connectomes (Takemura, Bharioke, et al., 2013; Shinomiya, Karuppudurai, et al., 2014; Takemura, Nern, et al., 2017; Shinomiya, Huang, et al., 2019). Notably, the connectome is not yet complete, and lacks mapping of many wide-field neurons spanning multiple eye columns. The main chemical synaptic inputs to T4 are medulla intrinsic neurons Mi1, Mi4, Mi9, transmedullary neuron Tm3, wide-field neuron CT1, transmedullary Y neuron TmY15, centrifugal neuron C3, and the T4 neurons of the same directional preference. The T5 neurons receive input mainly from transmedullary neurons Tm1, Tm2, Tm4, Tm9, Tm23, wide-field neuron CT1, transmedullary Y neuron TmY15, lobula tangential neuron LT33, and T5 neurons of the same directional preference.

Because T4 and T5 neurons encode local motion, analysis are often restricted to columnar neurons with clear retinotopy. In the ON motion pathway, L1 feeds into Mi1 and Tm3, and L3 feeds into Mi9. Lamina signals from L1 and L3 are carried to Mi4 trough L5 and Mi9, respectively. And CT1 connecting to T4 in the medulla receives lamina inputs indirectly via Mi1 and Mi9. In the OFF motion pathway, L2 feeds into Tm1, Tm2, and Tm4, and L3 feeds into Tm9. Again, CT1 receives only indirect lamina inputs in the lobula through Tm1 and Tm9.

There is a spatial organization in the pathways. The dendrites of T4 receive inputs from three sequential cartridges, e.g., visual points in space. From the tips to the base of the dendrite inputs to T4 come from: Mi9, next from Mi1 and Tm3, and finally from CT1, C3, and Mi4. Similarly, from tips to base of T5 dendrites inputs come from: Tm9, then Tm1, Tm2, and Tm4, and finally from CT1 (Shinomiya, Huang, et al., 2019).

\section{Physiology of motion pathways}

Because many inputs to DS neurons in the fly were mapped just recently, few cell types have been studied physiologically. Notably, all neurons described here transmit information through graded potentials, rather than spikes.

Prior attempts to map the circuits of motion detection focused on identifying two neurons that could fit into the motion detection models (section 1.3). But this assumes one of the models is true for the fly visual system. Besides, implementation at the algorithmic level does not necessarily map one-to-one to the neural level. Therefore, it is more 
beneficial to narrow down the particular algorithmic implementation of motion detection in Drosophila melanogaster before deciphering the circuit mapping at cell type resolution (Krakauer et al., 2017). Following this perspective, we discuss first the physiological properties of DS neurons, as well as their implications for algorithms of motion detection.

\section{Physiology of direction selective neurons}

Apart from the selectivity to ON or OFF moving edges, T4 and T5 share some common response properties. T4 and T5 are orientation-selective (Maisak et al., 2013; Fisher, Silies, and Clandinin, 2015). They are direction-opponent, i.e, they are excited by movement in their preferred direction, but inhibited by movement in their null direction (Badwan et al., 2019). They respond to illusory motion, or reverse-phi, that consists of sequential stimuli of opposite contrasts that elicit a percept of motion in the opposite direction of the sequence (Anstis, 1970; Salazar-Gatzimas, Agrochao, et al., 2018). T4 and T5 neurons incorporate information about ON and OFF stimuli (Leong et al., 2016; Salazar-Gatzimas, J. Chen, et al., 2016; Strother et al., 2017; Arenz et al., 2017; Salazar-Gatzimas, Agrochao, et al., 2018). As confirmed by connectomics, $\mathrm{T} 4$ and $\mathrm{T} 5$ receive inputs from at least three visual locations (Haag, Arenz, et al., 2016; Haag, Mishra, and Borst, 2017). Also T4 and T5 calcium imaging studies revealed both null direction suppression and preferred direction enhancement in both neurons (Fisher, Silies, and Clandinin, 2015; Haag, Arenz, et al., 2016; Haag, Mishra, and Borst, 2017). However, voltage recordings concluded that direction selectivity in T4 and T5 can emerge from linear input summation, and upstream nonlinearities only shape the already direction-selective signal (Wienecke, Leong, and Clandinin, 2018; Gruntman, Romani, and Reiser, 2018). Taken together, these constraints imply that models should: integrate inputs from three points in space with delayed processing of the extreme points with respect to the middle, combine both ON and OFF stimuli linearly in voltage, and potentially include dynamic nonlinearities (Badwan et al., 2019).

\section{Physiology of inputs to direction selective neurons}

Physiological studies aimed at deciphering the circuit implementation of motion detection. Rather than taking a historical account, here, we describe the physiological properties of the main synaptic inputs into T4 and T5. 
The pathways converging onto T4 and T5 split in the lamina neurons, however all lamina neurons L1, L2, and L3 depolarize to light decrements (OFF), and hyperpolarize to light increments (ON). They have different kinetics, L3 responses are more sustained than those of L1 and L2 (Reiff et al., 2010; Clark et al., 2011; Freifeld et al., 2013; Silies et al., 2013; H.H. Yang, St-Pierre, et al., 2016).

Downstream in the medulla, we have Mi1, Tm3, Mi4, and Mi9 as main inputs to T4. All depolarize to ON, except Mi9 that depolarizes to OFF (Behnia et al., 2014; Arenz et al., 2017; Strother et al., 2017). On the T5 pathway, all main inputs Tm1, Tm2, Tm4, and Tm9 depolarize to OFF (Behnia et al., 2014; Fisher, Leong, et al., 2015; Serbe et al., 2016; H.H. Yang, St-Pierre, et al., 2016; Arenz et al., 2017). These neurons have different temporal filtering properties. In the T4 pathway, Mi1 and Tm3 can encode higher temporal frequencies than Mi4 and Mi9. In the T5 pathway, Tm1, Tm2, and Tm4 encode similarly higher frequencies than Tm9 (Arenz et al., 2017). About their spatial properties, most neurons have center-surround receptive fields that match the optical resolution of one ommatidium, i.e., one pixel of the fly eye (Behnia et al., 2014; Serbe et al., 2016; Arenz et al., 2017). Interestingly, and opposite to other reports (Serbe et al., 2016; Arenz et al., 2017), Tm9 has been reported to have a wide receptive field spanning multiple columns (Fisher, Leong, et al., 2015). This wide receptive field indicates potential contributions from uncharacterized wide-field neurons in the fly eye (Nern, Pfeiffer, and Rubin, 2015).

\subsection{Thesis objectives}

Because of the cell type resolution of the studies, this discrepancy about Tm9 receptive field properties, sparked the interest in variability within cell types. This is particularly remarkable in the light of the regular and stereotypical constitution of the fly eye. Now, we have access to study cell type variability using an anatomically and molecularly defined cell type. Furthermore, we study this cell type under its computational context, i.e., motion computation. Tm9 is the major synaptic input to T5 DS neurons (Shinomiya, Karuppudurai, et al., 2014; Shinomiya, Huang, et al., 2019). The input from Tm9 to T5 is excitatory and cholinergic (Shinomiya, Karuppudurai, et al., 2014; Davis et al., 2019). Genetic silencing of Tm9 activity has profound effects in behavioral responses to OFF moving edges (Fisher, Leong, et al., 2015; Serbe et al., 2016).

Therefore, the aim of this thesis was to characterize the receptive field 
properties of Tm9, and their variability; to dissect the receptive field of Tm9 at the cell-type level; and to study the role of Tm9 and its input cell types into direction selective T5 neurons and optomotor behavior ${ }^{2}$. Each chapter of this thesis deals with each aim.

In the first chapter, we characterized Tm9 spatiotemporal response properties and their variability using a variety of visual stimuli and in vivo two-photon calcium imaging. To test the scope of cell type variability in the fly visual system, we further characterized response properties of other neuron types in the OFF motion pathway. We also recorded Tm9 and Tm4 simultaneously to reduce sources of variability across experimental conditions. We show Tm9 has variable properties, temporally $\operatorname{Tm} 9$ responses can be transient or sustained, whereas spatially receptive fields are narrow for OFF stimuli, and narrow to wide for ON stimuli. Furthermore, the variability is not common to all neurons tested.

In the second chapter, we studied the Tm9 receptive field contributions of the cell types that synapse onto Tm9. We first searched for novel inputs to Tm9 from wide-field cell types that could explain the wide ON receptive fields. We also performed a functional mapping of inputs to Tm9, using the candidate wide-field inputs together with connectomics data. We recorded Tm9 responses upon optogenetic activation of a variety of cell types, including some without direct connections. Then, we studied Tm9 response properties while we blocked the input from main lamina neurons, wide-field neurons, and electrical synapses onto Tm9. We show Tm9 is robust to single neurons manipulations. Moreover, we identified novel wide-field inputs that modulate Tm9 full-field responses, and the ON receptive field size and its variability.

Finally, in the third chapter, we studied the properties of T4 and T5 DS neurons while blocking activity of Tm9 neurons and its novel widefield inputs. We provide evidence that wide-field neurons modulate the response amplitude and direction-selectivity of T4 and T5 neurons.

Thus, the contributions of this thesis are threefold: study functional variability within cell types, analyze the cell type contributions to receptive fields, and define the role of wide-field neurons in fly vision, all within the context of a relevant neural computation, motion detection.

\footnotetext{
${ }^{2}$ Reflexive turning responses to moving stimuli, e.g., sinusoidal gratings.
} 



\title{
$-2-$ \\ Functional variability in classical genetically and anatomically defined cell types
}

\author{
"I learned very early the \\ difference between knowing the \\ name of something and \\ knowing something."
}

Richard P. Feynman

In this chapter, we study the receptive field properties of the main synaptic inputs to the direction-selective neurons T5 of the OFF motion pathway. We show functional variability in a genetically and anatomically defined cell type, namely Tm9. We also show how this variability can be modulated by inhibitory wide-field inputs.

First reports about the response properties of Tm9 stated Tm9 had wide receptive fields (Fisher, Leong, et al., 2015), but a following study reported narrow field properties (Serbe et al., 2016; Arenz et al., 2017). This discrepancy hinted at variability of functional properties within a cell type, which may be stimulus-specific, or may be more intrinsic to the neuronal properties. This chapter resolves the issue by studying the properties of Tm9 receptive fields. In the first section 2.1, we describe the variability in $\mathrm{Tm} 9$ receptive field properties. In the second section 2.2 , we test the specificity of the variability and possible underlying parameters by simultaneous dual imaging of $\operatorname{Tm} 9$ and another OFF pathway neuron, namely $\mathrm{Tm} 4$. In the third, final section 2.3 , we characterize and contrast the receptive fields of four main OFF pathway neurons independently: Tm1, Tm2, Tm4 and Tm9. 


\subsection{Functional variability in the main input to OFF direction selective neurons}

Tm9 is a crucial neuron for computing motion of dark moving edges. It is the main synaptic input to direction-selective T5 neurons in the OFF pathway (Shinomiya, Karuppudurai, et al., 2014; Shinomiya, Huang, et al., 2019). Fly optomotor behavior to dark moving stimuli is impaired upon Tm9 neurotransmission block, accompanied by decreased directionselectivity in neurons in the lobula plate (Fisher, Leong, et al., 2015; Serbe et al., 2016). Therefore, understanding the features encoded by Tm9 may bring us closer to crack the neural mechanism of motion computation in Drosophila melanogaster.

Tm9 is an anatomically defined cell type (Fischbach and Dittrich, 1989). Genetic lines to specifically label $\mathrm{Tm} 9$ are used in physiological and behavioral experiments (Fisher, Leong, et al., 2015). In transcriptomics, a single cluster is assigned to Tm9 (Konstantinides et al., 2018). Taken together, Tm9 is a genetically and anatomically defined cell type.

Usually, it is difficult to find such narrow anatomical definition and specific genetic access to a single cell type. As the morphology and genetic expression patterns seem homogeneous, one would expect the function to be equally constrained. The function of a neuron is key to understand neural computations. Downstream circuits will only receive the chemical or electrical signals encoding the preferred feature of the presynaptic neuron, not its morphology or full genetic makeup.

The discrepancy about wide versus narrow receptive fields of Tm9 can be have numerous sources: different environmental conditions for raising the flies, different adaptation states, the statistical structure of the stimuli, imaging conditions, among others. Another possibility is the variability in the functional properties of the cell type across and within individuals. This last hypothesis is amenable to testing, because carrying out the experiments in a single setup will reduce many sources of variability across laboratories. Thus, following section shows $\operatorname{Tm} 9$ functional characterization with a variety of stimuli. 


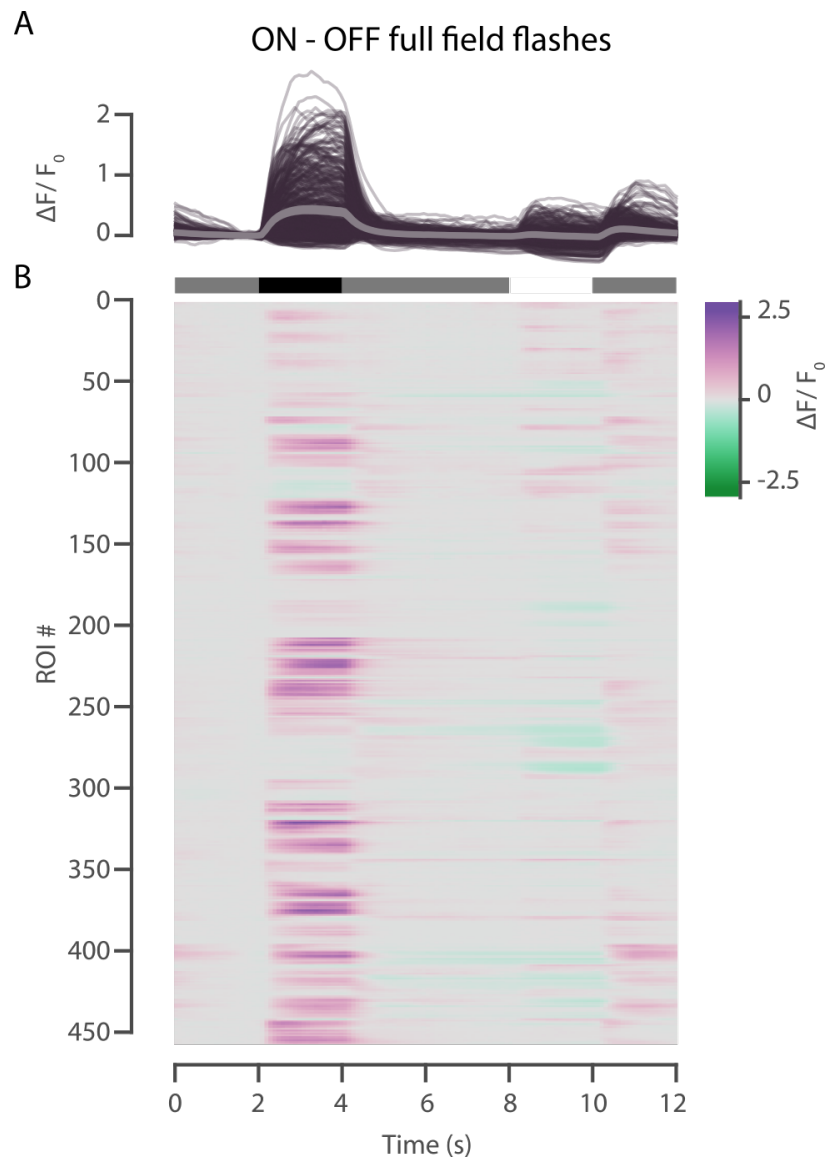

Figure 2.1: Tm9 responds preferentially to OFF full-field flashes.

A. Single Tm9 neuron responses to dark and bright full-field flashes of $2 \mathrm{~s}$ duration, interleaved by $4 \mathrm{~s}$ long intermediate gray screen. Mean trace and shaded standard error are overlaid. Tm9 neurons respond preferentially to OFF flashes. B. Single neuron traces in (A) stacked to highlight the variability of responses. Positive responses are encoded in purple, negative responses in green.

\section{Tm9 neurons responses to full field flashes}

A very simple functional division of neuronal properties is based on the response of a neuron to the onset or offset of a stimulus leading to ON, OFF or ON-OFF types. Here, we test Tm9 responses to full-field flashes of ON and OFF polarities; the screen goes darker or brighter relative to an intermediate gray screen. 


\section{Tm9 neurons response to full-field flashes is heterogeneous}

Neural responses were recorded using in vivo two-photon calcium imaging. Tm9 neurons expressed the genetically-encoded calcium indicator GCaMP6f (T.-W. Chen et al., 2013). We imaged the Tm9 axon terminals, located in the first layer of the lobula. Then, after image registration (motion correction), and manual selection of regions-of-interest(ROIs), signals were trial averaged. Fluorescence signal was normalized relative to the baseline fluorescence (mean signal of inter-stimulus background), per region-of-interest, resulting in $\frac{\Delta F}{F_{0}}$ (eq. 6.3). From here on, the terms region-of-interest and neuron will be used interchangeably, unless stated otherwise.

Responses of Tm9 neurons varied in kinetics and polarity. On average Tm9 neurons' calcium increased upon OFF stimulation, and remained unchanged or decreased slightly upon ON stimulation (fig. 2.1). Some neurons showed inverted polarity, i.e. their calcium levels increased upon ON stimulation.

Principal component analysis of full-field flashes responses To see finer structure in the responses we proceeded to visualize the data in a reduced dimensionality. We performed principal component analysis (PCA) on responses of the entire population of Tm9 neurons, without any quality control criteria. To focus on response kinetics and to prevent the response amplitude from dominating the variance, the principal component analysis was performed on z-scored data, i.e., subtracting the mean and dividing by the standard deviation of each neuron trace. The first principal component explained about $50 \%$ of the variance (fig. 2.2A).

The first five principal components that explained about $90 \%$ of the variance are shown in (fig. 2.2B). The first principal component resembled the average positive, sustained responses to OFF flashes, and a smaller negative response to $\mathrm{ON}$ flashes. The second principal component responded negatively to both $\mathrm{ON}$ and OFF. The third principal component was a positive, transient response to OFF and a positive response to the offset of the ON flash. The fourth principal component was mostly a transient, biphasic (sign changing) response to OFF. The fifth principal component was less intuitive.

Next, we examined the distribution of several response metrics on three dimensions, the first three principal components summing up to $70 \%$ explained variance. We first visualized the response quality index, a metric related to the trial variability. Low response quality indicates high trial variability, and vice-versa. From this, we got an intuition on 

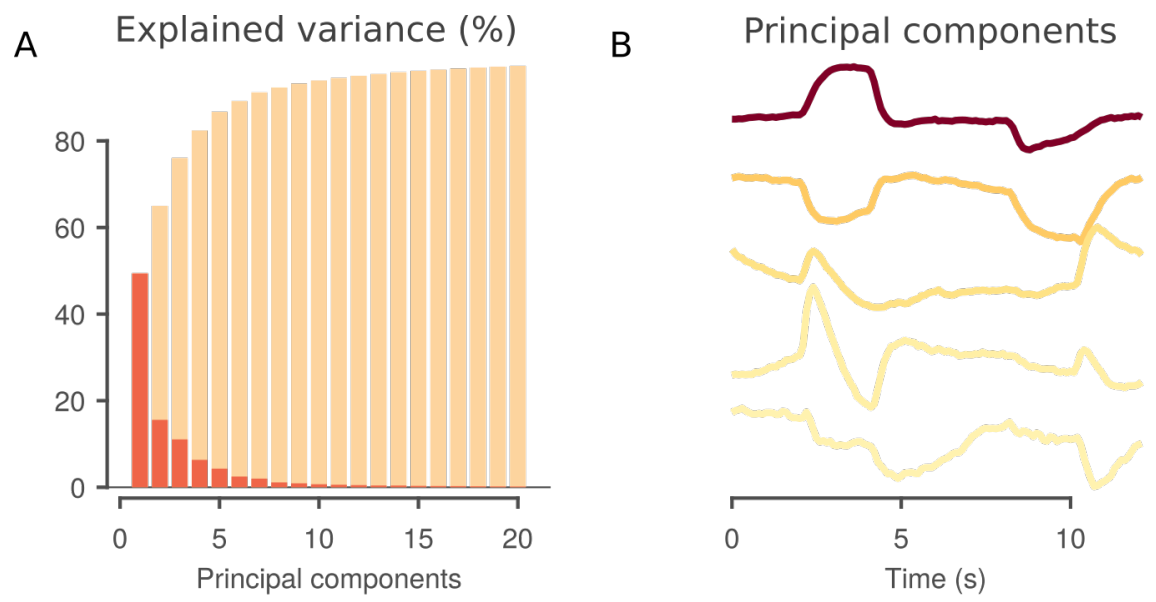

C Principal components 1 vs 2
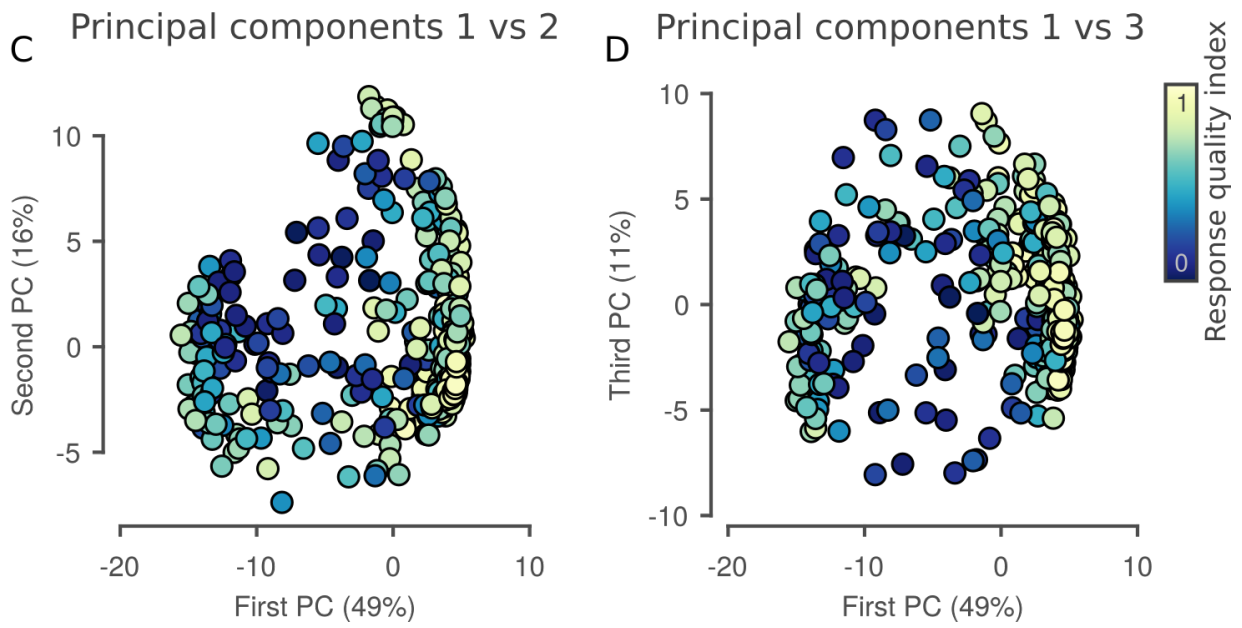

Figure 2.2: Principal component analysis of Tm9 ON-OFF full-field flashes responses.

A. Explained variance of each principal component in dark bars, light bars indicate the cumulative explained variance. B. Principal components in time coordinates, color coded by explained variance (lighter color for less explained variance). C-D. Visualization of first three principal components, color indicates response quality index; darker color is lower quality.

the distribution of responsive cells. In this low-dimensional projection, neurons spread elliptically, and neurons with higher response quality were in the outer region (fig. 2.2C-D). Thus, there was a structure defined by the response quality index, namely lower quality neurons occupied the central region. This indicated a response quality threshold for selecting responsive cells. This selection criterion is important for experimental conditions that may change the response properties of a neuron type, and 
to select among neuron types with different properties.

A

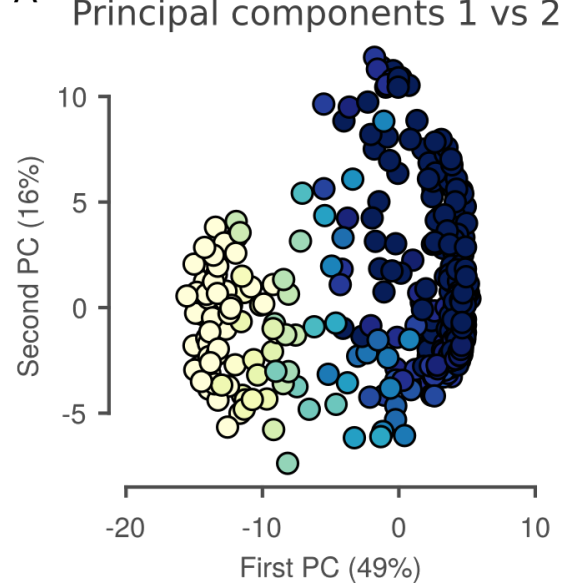

B

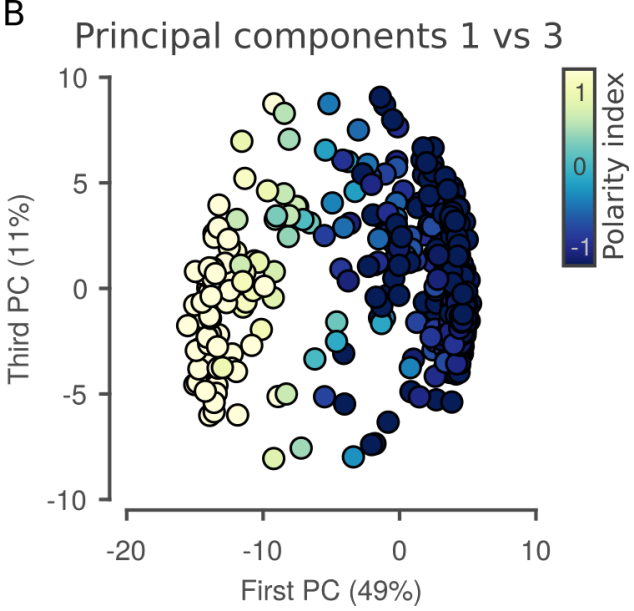

Figure 2.3: First principal component of ON-OFF responses relates to the polarity.

A-B. Data visualization from first three principal components, color indicates response polarity; darker color is OFF polarity, brighter colors are ON polarity.

Because the first and second principal components had opposite signs for the OFF stimulus, the first principal component could relate to the response polarity (eq. 6.7). To visualize this, we colored the points according to the polarity index, which is positive for $\mathrm{ON}$ and negative for OFF responses. Indeed, the response polarity was divided along the first principal component (fig. 2.3). The response polarity (first principal component) dominated most of the variability in the data. However, neurons that responded preferentially to ON stimuli, were likely to have portions of their receptive fields outside the stimulation area, and thus, the responses would be mostly driven by the inhibitory surround (Freifeld et al., 2013; Fisher, Leong, et al., 2015).

On the other hand, a variety of temporal profiles was obscured by averaging all responses (fig. 2.1). Looking carefully, some responses peaked earlier and decayed, while others rose more slowly and continuously with the stimulus. The first and second principal components indicated late peaking responses, and the third and fourth components peaked earlier, started decaying, and even changed sign during the end of the OFF stimulus (fig. 2.2B). Thus, Tm9 neurons showed temporal response variability, most were sustained and late peaking, and a smaller subset was more transient (early peaking and decaying). 
A

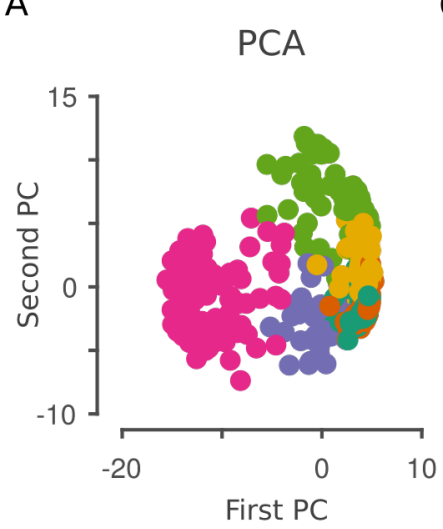

B

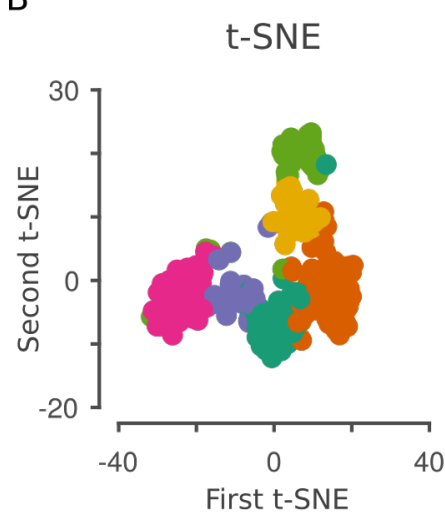

C Clusters of z-scored responses to ON-OFF
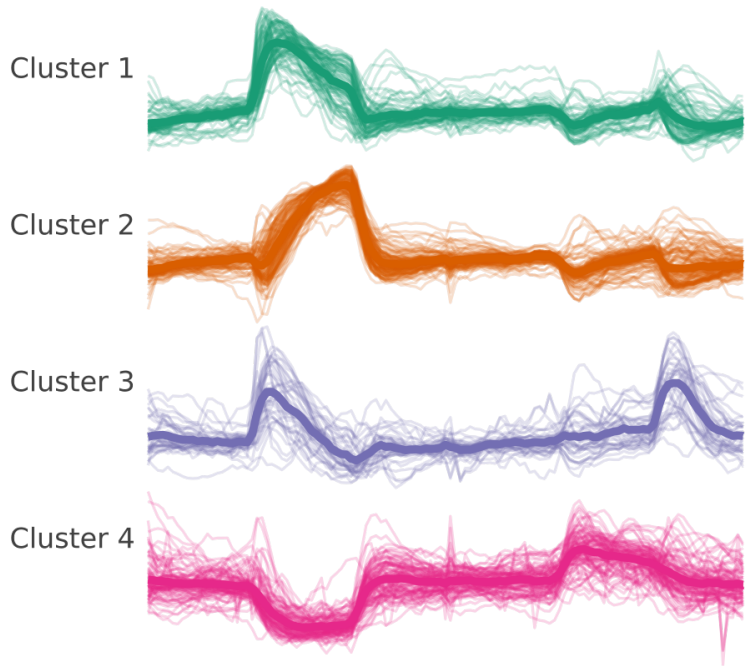

Cluster 5

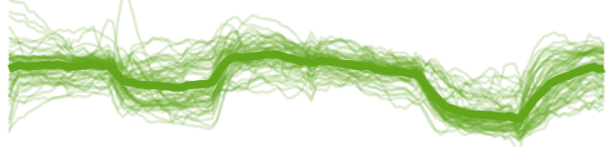

Cluster 6

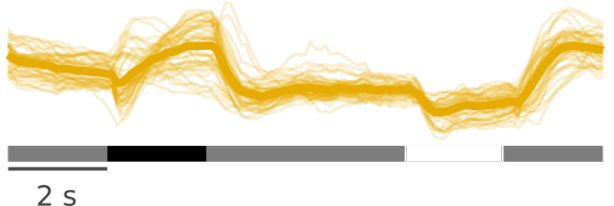

Figure 2.4: K-means clustering of different z-scored response dynamics.

A. Six clusters visualized on the first two principal components from fig. $2.2 \mathrm{~B}$, separation is not so clear for this linear method. B. Six clusters visualized on the nonlinear embedding from t-SNE, separation is clearer. Note that clustering was done independently of the dimensionality reduction techniques. C. Clustered z-scored responses: first and third clusters are more transient than second and sixth clusters. Cluster four follows the stimulus intensity. Cluster five responds negatively for both stimuli.

Clustering of full-field flashes responses To extract structure from different polarities and temporal response properties, we clustered the population responses using K-means. To reduce noise in the data, responses were approximated by the first 20 principal components. Again, to discard amplitude effects on the clustering, the data were z-scored by subtracting the mean and dividing by the standard deviation of the response of each neuron. The number of clusters was set to six, exploring grouping with 
hierarchical clustering also defined six clusters (data not shown).

We visualized the clustering in the first two principal components, which explained $65 \%$ variance. Clusters overlapped in this reduced representation (fig. 2.4A). Principal component analysis is a linear dimensionality reduction method, to check whether a nonlinear dimensionality reduction provided better cluster separation we used t-SNE (t-stochastic neighboor embedding). Principal component analysis defines a coordinate transformation that can be applied to new data. However, t-SNE is and embedding and to accommodate new data one needs to compute a new embedding. The meaning in the t-SNE components is not intuitive, and conventional distance metrics are not preserved. Hence, t-SNE is a useful visualization tool, but should not be used for further analysis. In the t-SNE, the clusters were more separated (fig. 2.4B).

The first and second clusters split the data into early and late peaking responses, respectively. The third cluster responded similarly to the OFF transitions: gray to dark, and bright to gray. The fourth cluster followed the stimulus intensity. The fifth cluster responded negatively for both $\mathrm{ON}$ and OFF stimuli. Finally, the sixth cluster responded positively to OFF and negatively to ON, and the OFF response was similar to the one of cluster two. Data from clusters three, four, and five were in the region of low response quality (fig. 2.2C-D).

We next related the clusters to conventional properties: polarity, sustenance, time to half extreme, time to extreme, and response quality index (see Section 6.7 for definitions). All properties were calculated for the OFF stimulus, except polarity that used ON and OFF portions, and response quality index that used the whole trace. All clusters but the fourth had negative polarity, i.e., preferred OFF flashes ${ }^{1}$. Clusters four and five had the lowest response quality, we regarded them as noise clusters. Clusters two and six were the most sustained and slowest to reach their extreme response. Conversely, clusters one and three were more transient, and peaked earlier (fig. 2.5). From the sustained clusters, both responded to OFF flashes, but only the sixth responded to the offset of ON flashes. Similarly, both transient clusters responded to OFF, but only cluster three responded to ON offset. Because the offset of ON flashes is an OFF flash, Tm9 shows mainly two robust types of response dynamics to OFF full-field flashes.

\footnotetext{
${ }^{1}$ Cluster four responded more negatively to ON than to OFF, thus according to our polarity index (eq. 6.7) it had OFF polarity.
} 

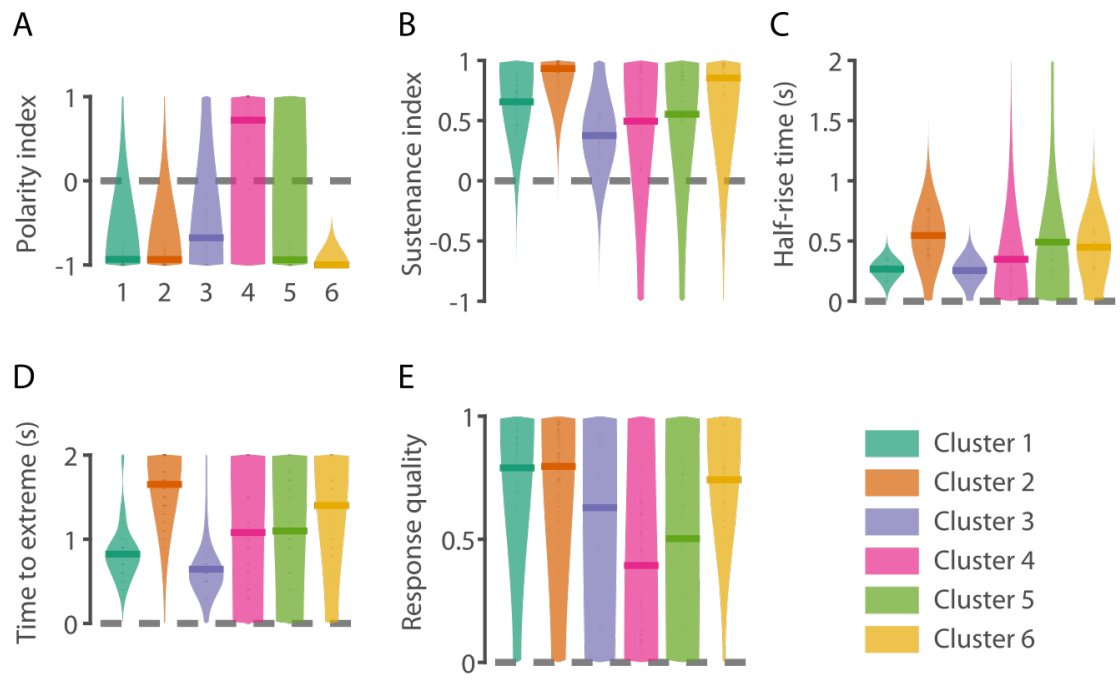

Figure 2.5: Response properties per cluster.

A. Polarity index: All clusters but the fourth are OFF selective. B. Sustenance index: Clusters two and six are sustained, while clusters one, three, four and five are more transient. C. Time to peak: clusters two and six reach half of their peak later than the other clusters. D. Time to peak: clusters two and six peak later than the other clusters.E. Response quality index: clusters four and five have the worst quality and can be considered noise clusters.

\section{Tm9 neurons responses to flashing bars}

The responses to full-field flashes depend on the integration of the stimulus by the receptive field of the neuron. We therefore next measured the receptive field components at a finer spatial resolution, similar to the resolution of one ommatidium $\left(5^{\circ}\right)$.

Previous studies of the spatial receptive field of Tm9 measured responses to OFF bars (Serbe et al., 2016), or the correlation of the stimulus and response (Fisher, Leong, et al., 2015; Arenz et al., 2017). Thus, the former ignored potential ON receptive field properties, while the later included both ON and OFF contributions in a non-obviously separable way. Here, we show the responses of Tm9 neurons to both ON and OFF bars, separately.

To measure the receptive field we used horizontal or vertical bars of $5^{\circ}$, on a square screen of $60^{\circ}$ side. Bars were presented for $1 \mathrm{~s}$ interleaved by $1 \mathrm{~s}$ of background. ON bars had maximum projector luminance on a background of minimum projector luminance. OFF bars had minimum luminance on maximum luminance background. A stimulus sequence 

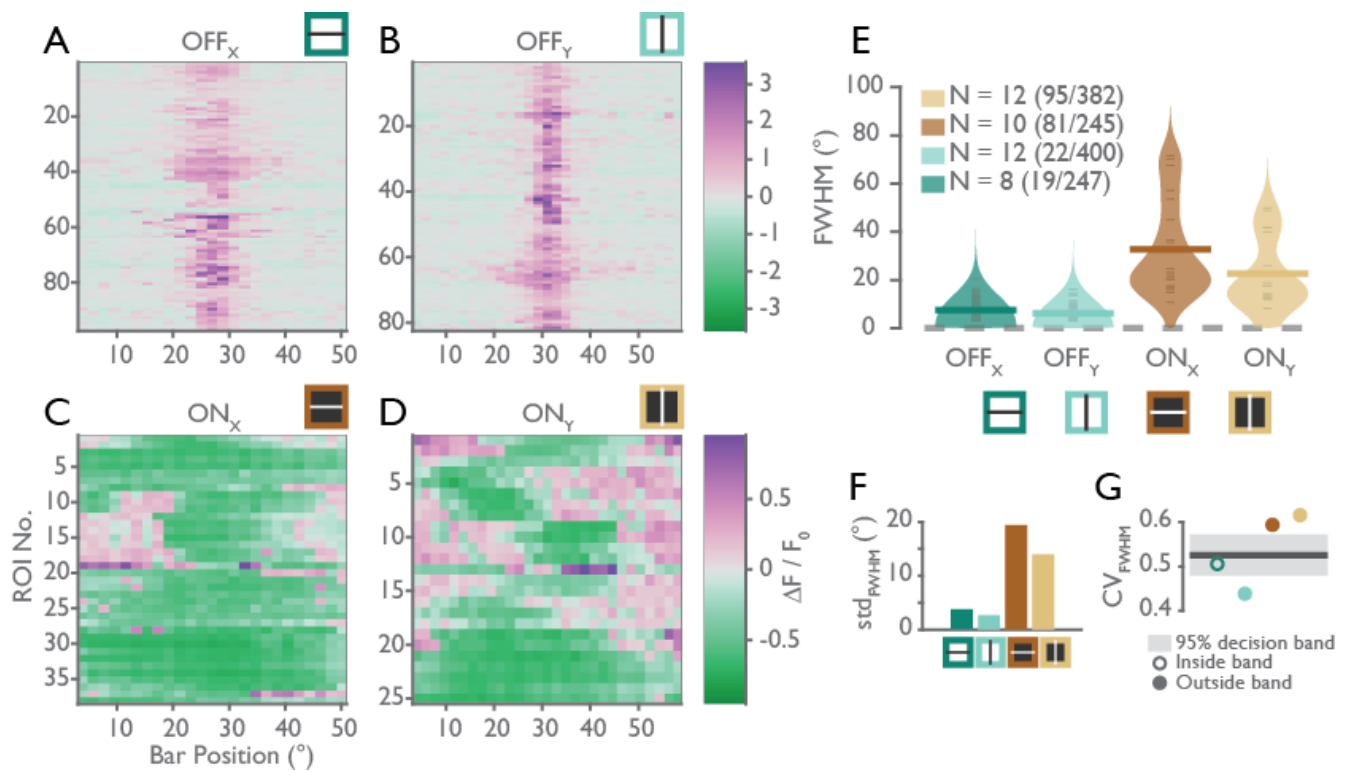

Figure 2.6: Tm9 receptive fields are variable in size, especially for ON stimuli.

A-D. Tuning curves for neurons that were responsive (response quality index $>0.5)$, and had a good fit $\left(r^{2}>0.3\right)$, neurons were analyzed independently for each stimulus. Responses in purple are positive, green ones are negative. A, B. OFF receptive fields for horizontal (A), and vertical (B) bars. C, D. ON receptive fields for horizontal (C) and vertical (D) bars. E. Receptive field full width at half maximum (FWHM) for data in A-D. ON receptive fields are larger than OFF receptive fields. F. Variance of FWHM distributions in E. ON receptive field sizes are more variable than OFF ones. G. Coefficient of variation (CV) of FWHM distributions in E. Also shown are the central decision line and bootstrap decision band at 95\% confidence. Values outside the band (filled circles) are significantly different from the population, values within the band (empty circles) are not. ON receptive fields are more variable than the average across stimuli. $\mathrm{N}$ is the No. of flies, in parenthesis are (No. of used neurons / No. of recorded neurons).

consisted on a single polarity and a single orientation. A trial consisted on the presentation of all bar positions shuffled for a pseudo-random presentation. Several trials (four to six) were presented for each stimulus.

We constructed the tuning curve of the neuron using the extreme value of the $1 \mathrm{~s}$ trace for each bar position ${ }^{2}$. To quantify its spatial extent a single

\footnotetext{
${ }^{2}$ The extreme of the response was the value that had maximum absolute value. Other
} 
1D Gaussian was fitted to the tuning curve (eq. 6.8). The full width at half maximum (FWHM) of the Gaussian (eq. 6.9) was used as the receptive field size. Only ROIs satisfying quality criteria set in 6.6 were used for further analysis (fig. 2.6).

To visualize the trial-averaged tuning curves we color coded the response, positive values in purple and negative values in green. Tm9 neurons responded to both $\mathrm{ON}$ and OFF bars (fig. $2.6 \mathrm{~A}-\mathrm{D}$ ). The terms $\mathrm{ON}$ and OFF receptive field refer to tuning curves from ON and OFF bars, respectively. Same holds for vertical and horizontal conditions. OFF receptive fields are narrow, about $10^{\circ}$ for both orientations (fig. 2.6 A-B, E). ON receptive fields are wider, ranging from $10^{\circ}$ to $70^{\circ}$ (fig. $2.6 \mathrm{C}-\mathrm{D}, \mathrm{E}$ ). The vertical receptive fields were smaller than horizontal ones, more so for ON bars. Taken together, Tm9 has variable receptive field properties, narrow for OFF stimuli and narrow to wide for ON stimuli.

We quantified variability in the receptive field size using the standard deviation and the coefficient of variation (fig. 2.6 F-G). The standard deviation measures the spread of the data, but its value depends on the average magnitude, and units of the data. The coefficient of variation (CV) accounts for this by taking the ratio of the standard deviation to the mean, a dimensionless value that can be expressed as a percentage. Thus, the coefficient of variation can be used to compare data dispersion across properties measured on a ratio scale, i.e., a scale with a unique and non-arbitrary zero value. The standard deviation of the receptive field size was larger for ON stimuli than OFF stimuli, and for horizontal than vertical bars (fig. $2.6 \mathrm{~F}$ ). The coefficient of variation of the receptive field size was larger for ON than for OFF stimuli (fig. $2.6 \mathrm{G}$ ). To test whether the difference in variability was statistically significant, we bootstrapped a confidence interval for the mean coefficient of variation of all stimuli (Boiroju and Reddy, 2012). Values outside the band are statistically significant differences at $95 \%$ confidence. ON receptive fields are more variable than OFF receptive fields (fig. $2.6 \mathrm{G}$ ). Therefore, Tm9, a well-defined cell type, showed functional variability, which is higher for ON stimuli.

\section{Responses of Tm9 neurons to full-field noise vs ON-OFF full-field flashes}

So far, we showed Tm9 functional variability: temporal variability to a global stimulus (2.1), and spatial variability to a localized stimulus

options included the mean or median response that are more robust to outliers, but underestimate transient responses. 
(2.1). We then asked whether the variability extends to stimuli that have both temporal and spatial components. We first extended the temporal complexity of full-field flashes, using a ternary noise stimulus. The screen could take randomly every $50 \mathrm{~ms}$ one of three values: dark, gray, or bright. Using reverse correlation analysis, the receptive field of the neuron was estimated as a linear filter from the responses to the stimulus. This linear filter described the temporal dynamics of Tm9 neurons. To extract and compare multiple properties, we recorded responses of every neuron to all stimuli, including previous stimuli: ON-OFF full-field flashes and flashing bars.

First, we analyzed the responses to ON-OFF full-field flashes and compared the properties to the previous dataset. We recorded fewer flies and neurons, but two clusters with different response kinetics remained, clusters one and three (fig. 2.7 C, G-I).

We then analyzed the responses to full-field noise. We modeled the stimulus-response function of the neuron as a linear equation: the response equals the stimulus design matrix (stimulus time history) multiplied by the linear (temporal) filter of the neuron. To obtain the temporal filter, the equation was inverted using ordinary least-squares. Thus, the temporal filters were a linear approximation to the response function of the neuron to full-field stimuli.

To quantify how good this approximation is, we calculated the filters using $80 \%$ of the response time series, and predicted the response of the remaining $20 \%$ of the time series. The quality of the filter approximation was defined as the correlation between the predicted and the actual response. However, we did not discard any data.

Most of the filters had negative sign (fig. 2.8A). Because the response is obtained by multiplying the filter and the stimulus, a negative filter yields a positive response to negative (dark) stimuli, and negative response to positive (bright) stimuli. We observed a variety of filters. Like in our approach to ON-OFF full-field flashes, we clustered the responses using k-means, and found that six clusters separated the data visually (fig. 2.8B). The number of clusters (6) was in the order of the number of flies recorded (10). To test if the clusters arise from variability within or across flies, we colored the neurons in each cluster according to the fly they belong to. We also shifted the traces vertically and randomly (jittered them) for better visibility. Most clusters included neurons from more than one fly, and for most flies the neurons distributed among different clusters. Hence, the clusters represented variability across and within individuals (fig. 2.8B).

The temporal filters can be described by the frequencies they encode. We calculated the frequency spectrum of every filter using the FFT (fast 
Fourier transform), and plotted the amplitude of the transform, i.e., the amplitude spectrum (fig. 2.8C). The clusters were similar in the frequency domain. Only the cluster six (yellow) had longer tails and peaked at a higher frequency. Correspondingly, this cluster had narrower filters and peaked closer to response than others (fig. 2.8C-D). On the other hand, cluster two (orange) peaked later and the spectrum decayed faster. Overall, the filters do not encode frequencies higher than about $5 \mathrm{~Hz}$. Tm9 is a band-pass for low frequencies.

To compare the properties of this dynamic stimulus to the simpler ONOFF full-field flashes we used the time to peak of the linear filters. The time to peak was the time of the minimum filter value, because the filters were mostly negative. The time to peak was differed gradually between clusters (fig. 2.8D), clusters with similar time to peak had different filter widths, e.g., clusters four (pink) and five (green). We correlated the time to peak of the linear filter with the following properties from all neurons in fig. 2.7: mean response to ON and OFF flashes, polarity index, sustenance index, and time to extreme (peak). The temporal properties of ON-OFF full-field flashes were correlated with each other, namely sustenance index and time to extreme. Both of those properties correlated negatively with the filter time to peak (fig. 2.8E). So more sustained neurons, which also peaked later for ON-OFF full-field flashes, had longer absolute times to peak for temporal filters. Thus, some temporal properties of Tm9 responses to full-field stimuli of different dynamics are correlated. 
A

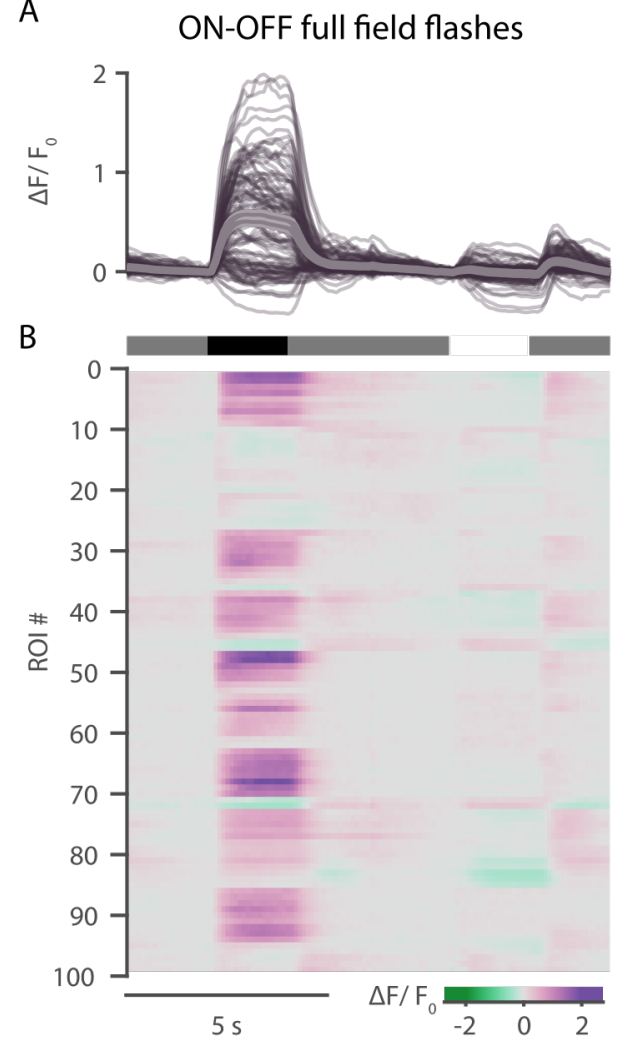

D

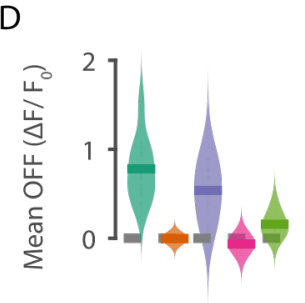

$\mathrm{E}$
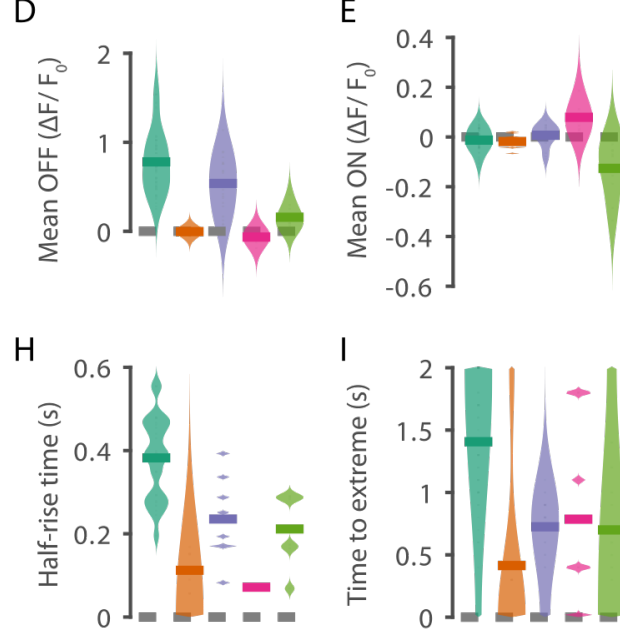

$c$

\section{Clusters of ON-OFF responses \\ Colored by:}

Cluster index

Fly index
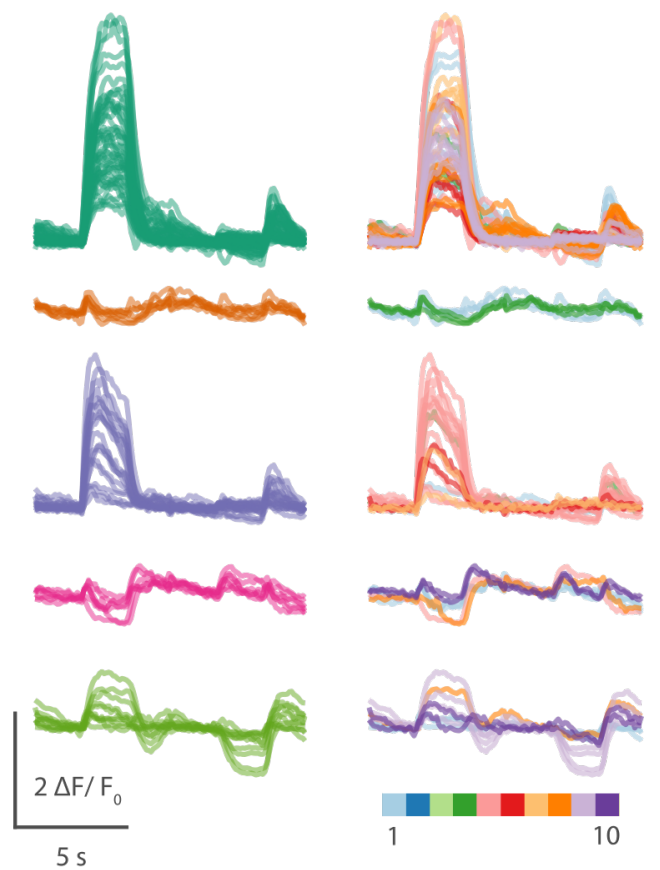

$\mathrm{F}$
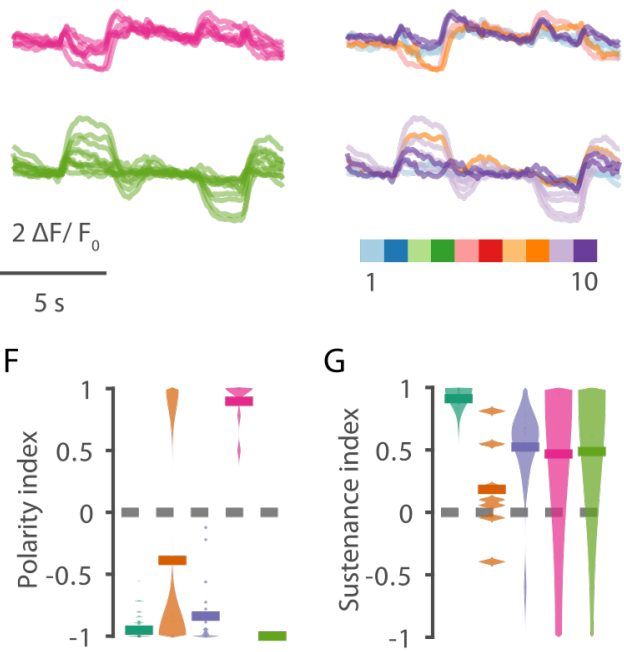

G

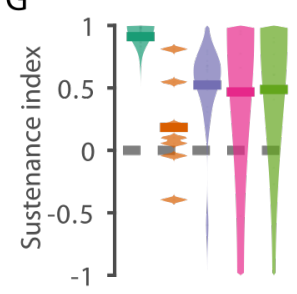

Figure 2.7: Two response modes are reproducible and distribute over flies.

A. Tm9 responses to ON-OFF full-field flashes. B. Responses in (A) stacked to highlight single cell traces. C. K-means clustering of different z-scored response dynamics from A-B: left are responses grouped and colored by cluster index, right are response clusters colored by fly index. D-J. Response properties from clusters in $\mathrm{C}$. 
A Temporal linear filters

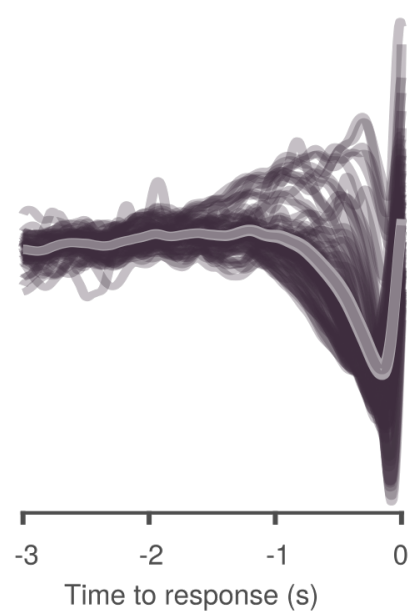

C

B Linear filters Linear filter clusters

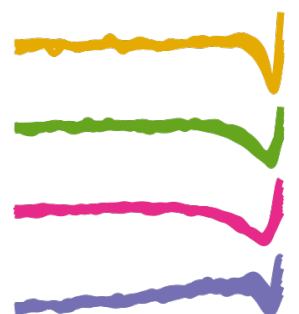
labeled by fly
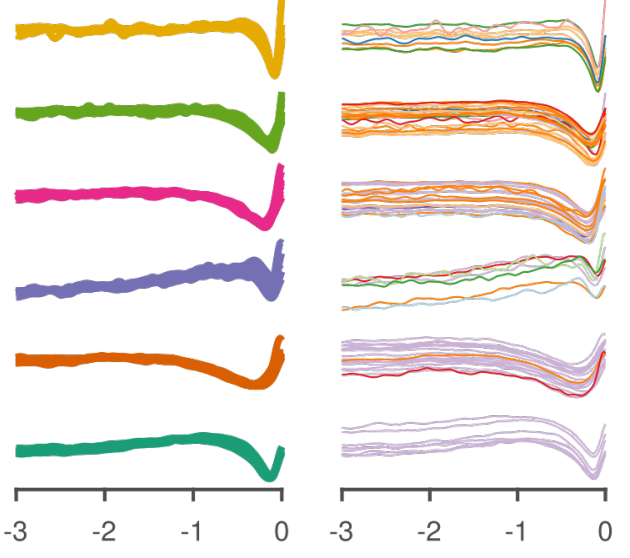

$E$

Correlation of full-field properties amplitude spectrum
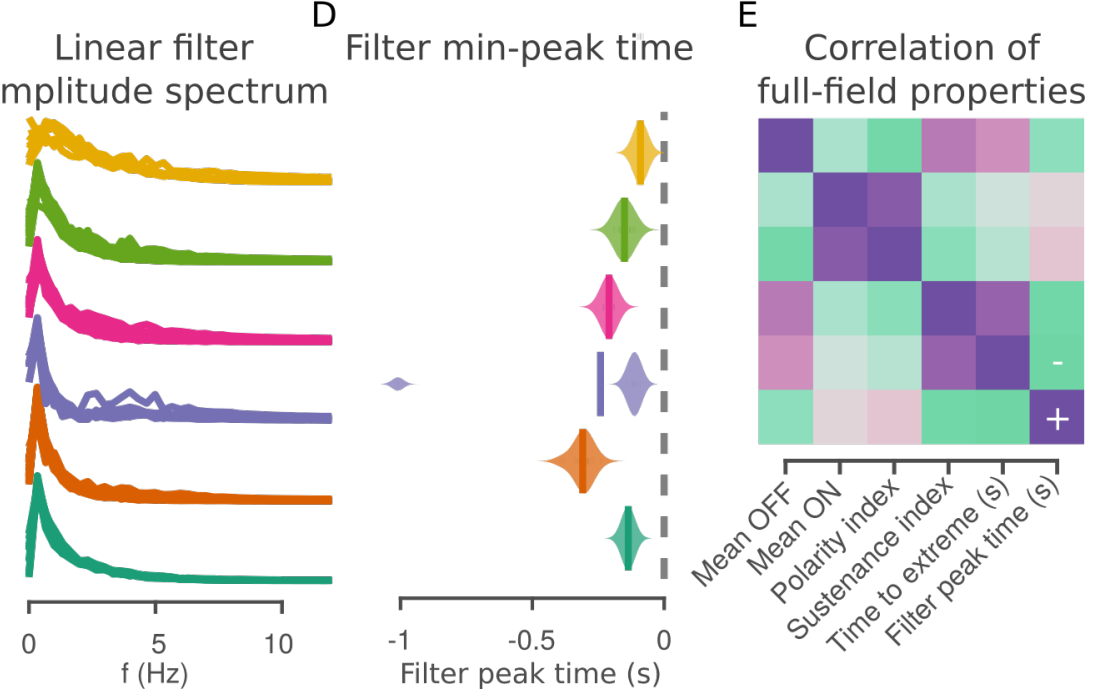

Figure 2.8: Tm9 temporal filter variability distributes over flies.

A. Tm9 linear temporal filters from responses to full-field noise, time of response is at zero. B. K-means clustering of different z-scored response dynamics from A: left are responses grouped and colored by cluster index, right are response clusters colored by fly index. C. Amplitude of the Fourier transform of the linear filters in B. The narrower filters can encode higher frequencies than wider filters (yellow vs orange). D. Time to the filter peak (minimum value) for clusters in $\mathrm{B}$. The increase in peak times in gradual, and clusters with similar peak time have different peak widths (not quantified). E. Rank correlation of peak times of linear filters with response properties from ON-OFF full-field flashes in fig. 2.7. Filter peak time correlated with ON-OFF full-field flashes sustenance index $(-0.40)$ and time to peak (-0.42). Because filter times are negative, more negative times to peak for filters mean slower filters. Negative correlation between filter peak time and sustenance means slower filters have more sustained responses. 


\section{Responses of Tm9 neurons to noise bars vs flashing bars}

We saw temporal variability in Tm9 responses to full-field stimuli with different temporal scales. We also saw variability in spatial stimuli with flashing bars. We proceeded to test if the spatial variability also extended to more complex and dynamic stimuli. Again, we used a ternary noise stimulus, but this time the screen was divided into bars $5^{\circ}$ wide. For every frame a random luminance value was drawn independently for each bar, frames were updated every $100 \mathrm{~ms}$. We extracted the filter similarly to the full-field one, by using least-squares to invert the linear equation. In this case, the filter had both a spatial and a temporal dimension. The spatiotemporal filters, will be referred to as spatiotemporal receptive fields (STRFs) interchangeably. The population of recorded filters for vertical and horizontal noise bars is shown in fig. 2.9.

To compare the properties of these filters with the previous stimuli, the filters were split into a spatial and a temporal filter. We did so by first locating the extreme value of every filter from fig. 2.9. From this point the temporal and spatial filters were extracted as the profiles along the temporal and the spatial axis, respectively.

\section{Comparison of temporal filters}

First, we compared the temporal filters across stimuli: vertical and horizontal noise bars, and full-field noise (fig. 2.10). Temporal filters for horizontal and vertical noise bars were similar (fig. 2.10A-B). Unlike temporal filters for noise bars, some temporal filters for full-field noise had positive incursions before the negative peak (fig. 2.10C). To compare the filters in more detail, we correlated them.

To distinguish contributions of good versus bad filters, we colored them by their explained correlation ${ }^{3}$, using the minimum explained correlation of the three filters per neuron. Filters were correlated with each other in the same amount, because the bootstrapped confidence interval of the correlations overlapped (fig. 2.11). Thus, the temporal properties measured for different orientations and spatial structures were similarly correlated.

\section{Comparison of spatial filters}

Receptive field visualization We then visualized the spatial filters extracted for noise bars and the tuning curves from flashing bars. To get

\footnotetext{
${ }^{3}$ Not to be confused with the correlation between different filters calculated here.
} 
A Tm9 spatiotemporal receptive fields (STRFs)

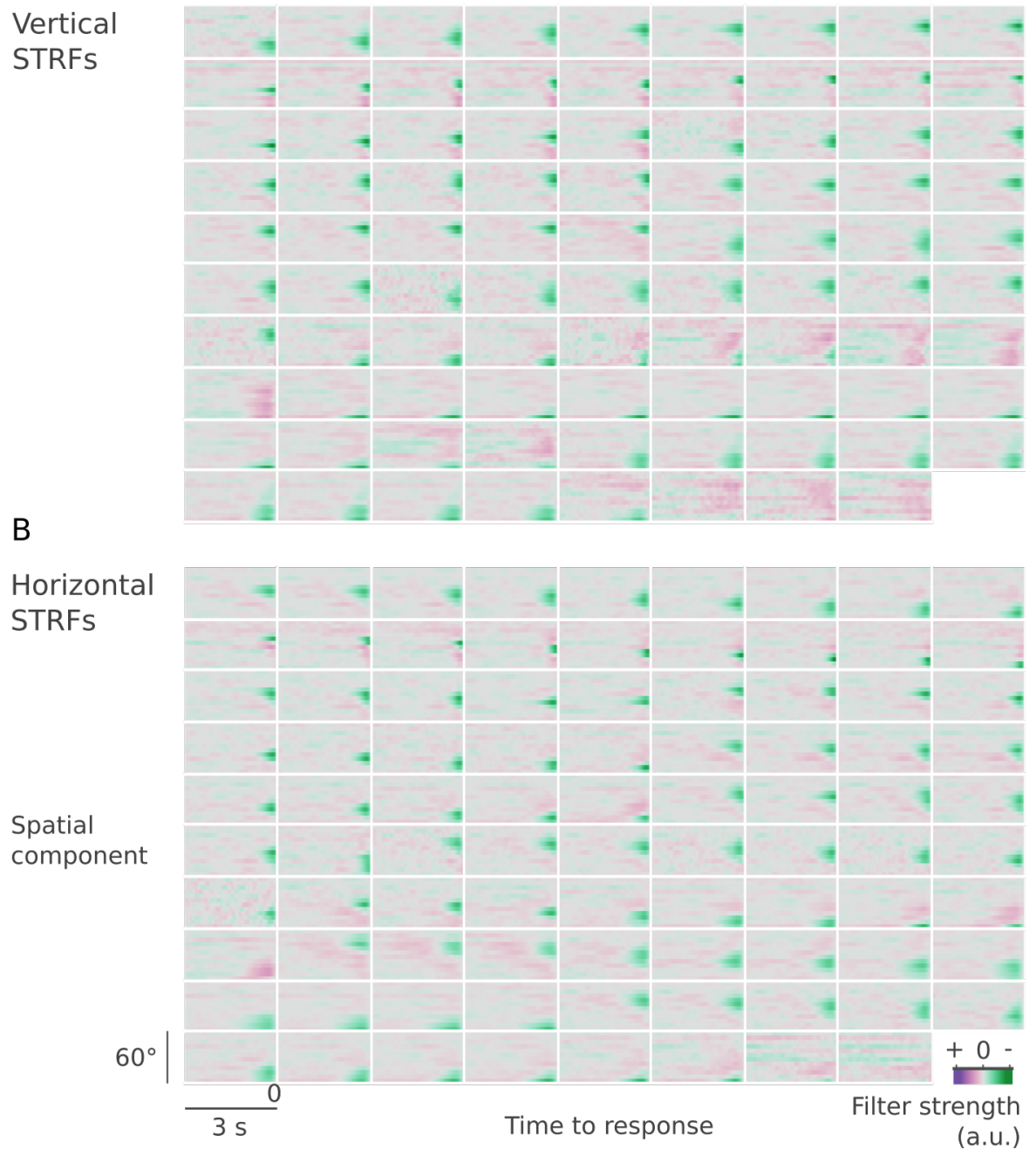

Figure 2.9: Tm9 spatiotemporal receptive fields.

Tm9 spatiotemporal filters obtained from Tm9 responses to ternary noise bars. A. Filters for vertical bars. B. Filters for horizontal bars. Positive filter values are purple, negative values are green. Every rectangle corresponds to one neuron, same neurons are shown in A and B. Time of response at zero is on the left edge.

an image of the receptive field of a neuron in the screen, we calculated the Cartesian product of the tuning curves (or spatial filters) from the two orthogonal stimulus orientations. We color coded this product in both magnitude and sign of the receptive field components. The product magnitude was linearly encoded in the luminance of the colors. To discern the 


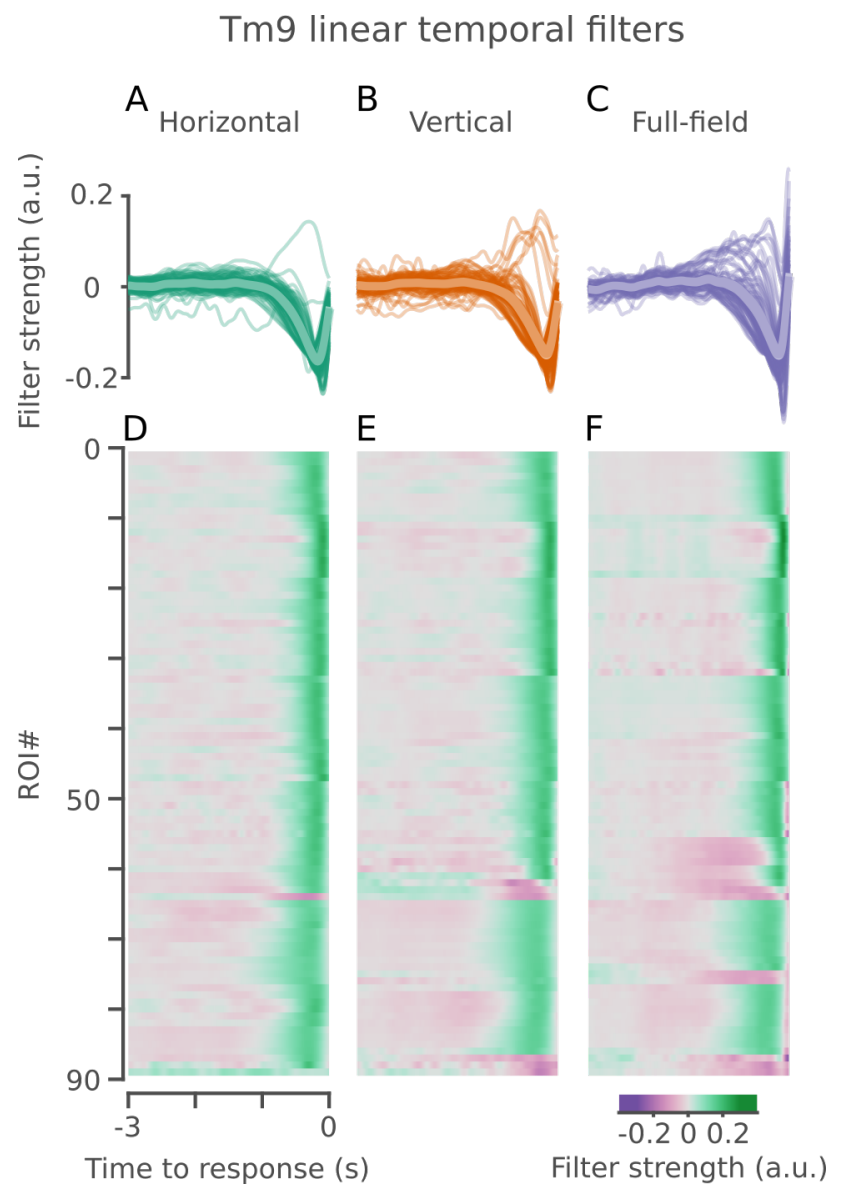

Figure 2.10: Tm9 linear temporal filters.

A-C. Linear temporal filters for Tm9 neurons, single neuron traces, mean and standard error overlaid. Filters for (A) horizontal noise bars, (B) vertical noise bars, and (C) full-field noise. D-F. Same as A-C but highlighting single neuron dynamics. Some full-field filters in (C, F) had positive values before negative peak, and positive values at response time.

sign of the product components we used three colors. To reduce saliency of locations where product components had opposing signs, we colored them gray. We colored in yellow (orange / brown) regions where both components were negative. And we used green for regions where both components were positive. ${ }^{4}$ Thus a mix of green and yellow, indicated mixed sign contributions, e.g., excitatory and inhibitory center-surround components of the receptive fields. This mix was most prominent in re-

\footnotetext{
${ }^{4}$ These colors were meant to be color-blind friendly for most options tested on (Coblis - Color Blindness Simulator - Colblindor 2019).
} 


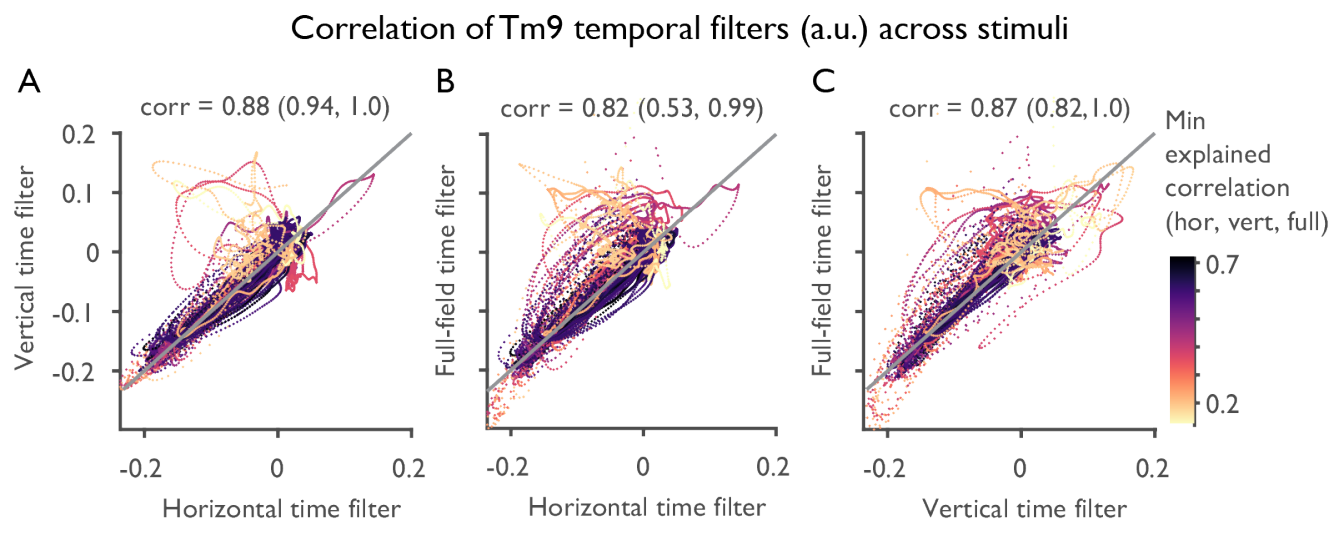

Figure 2.11: Tm9 linear temporal filters correlate accross stimuli.

A-C. Correlation of linear temporal filters for Tm9 neurons: scatter plot and correlation with $95 \%$ bootstrapped confidence interval in parenthesis. Filters were color coded by the minimum explained correlation of the three filters per neuron (darker color means higher explained correlation). Comparison for (A) horizontal vs vertical noise bars, (B) horizontal noise bars vs full-field noise, and (C) vertical noise bars vs full-field noise. Filters were correlated across stimuli, and filters with higher explained correlation laid closer to identity line (gray).

ceptive fields with the smallest centers, like the second column of each panel in fig. 2.12 A-C. Moreover, one can see the retinotopy, different Tm9 neurons covered different portions of visual space, e.g., first column in fig. $2.12 \mathrm{~B}$.

To compare receptive field sizes across stimuli, we fitted a Gaussian to each receptive field component for every stimulus. The size of the receptive field was again the full width at half maximum of the Gaussian. The receptive field sizes were mostly correlated between orientations of the same stimulus, rather than between stimuli. Thus, we averaged the full width at half maximum for vertical and horizontal receptive fields. Mean FWHM of receptive fields were $13^{\circ}$ for $\mathrm{OFF}, 23^{\circ}$ for $\mathrm{ON}$, and $17^{\circ}$ for noise bars, respectively. Receptive fields were largest for $\mathrm{ON}$ flashing bars, and smallest for OFF bars (fig. 2.12D-E). The properties measured with noise, which mixes $\mathrm{ON}$ and OFF contributions, were intermediate to the ones measured with pure ON or OFF contributions. We conclude that the wide-field response properties observed for different stimuli arise from the ON portion of the receptive field. 


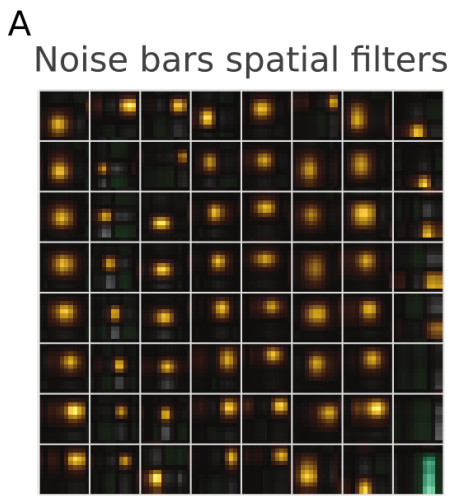

$B$

OFF bars spatial filters

C

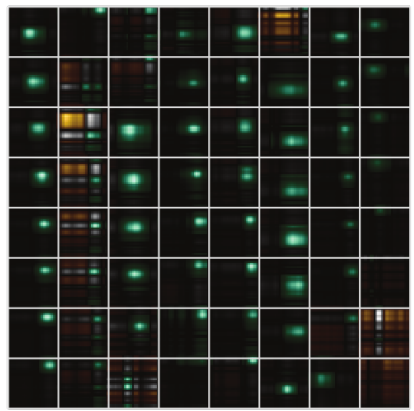

ON bars spatial filters

D

Full width at half maximum compared across stimuli
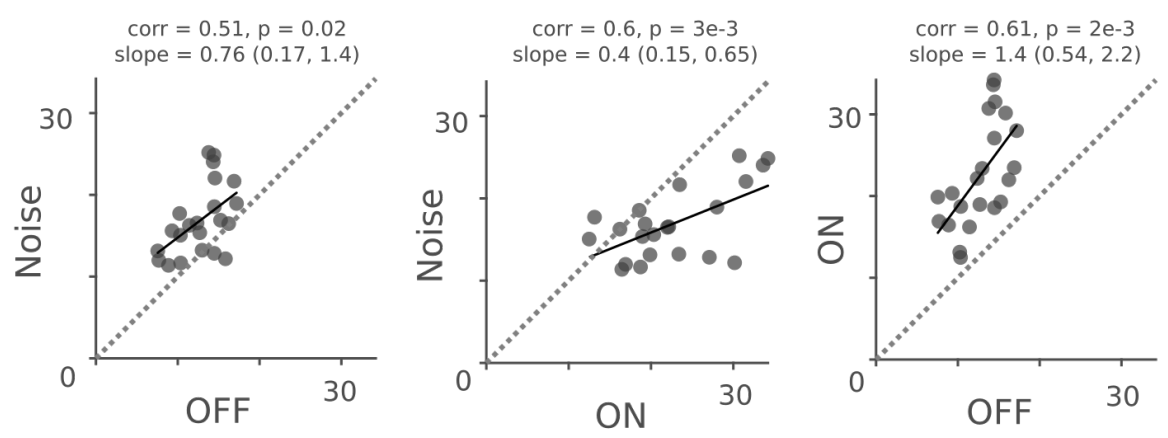

$\mathrm{E}$

$\mathrm{F}$
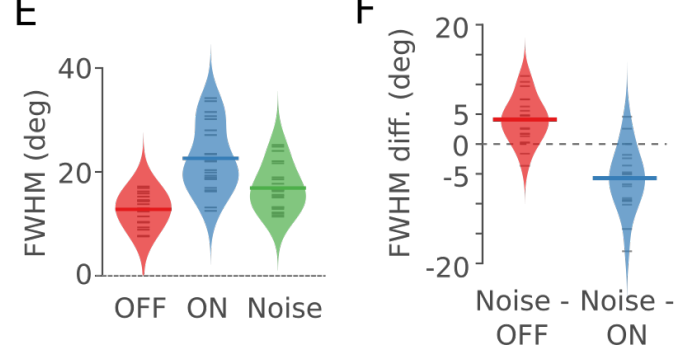

Figure 2.12: Noise receptive fields are larger than OFF and smaller than $\mathrm{ON}$ receptive fields.

A-C. Tm9 receptive fields visualized as Cartesian product of tuning curves for vertical and horizontal bars, for ternary noise bars (A), OFF bars (B), and ON bars (C). D. Receptive field sizes (FWHM) compared across stimuli: Noise vs OFF, Noise vs ON, and ON vs OFF. The scatter plot shows the mean of the FWHM of vertical and horizontal stimuli, only neurons with good fits $\left(r^{2}>0.5\right)$ are included. On top: correlation between stimuli widths with p-value and slope of linear fit (dark line) with 95\% confidence interval. Receptive field sizes were correlated across stimuli. E. Distribution of receptive field sizes from data in D: shape is the density of data distribution, single values are shown as small lines, wider line is at the mean value. F. Comparison of receptive field sizes: Noise minus OFF and Noise minus ON. Both are significant (tab. 2.1). 


\subsection{Simultaneous receptive field measurements of two neuron types}

In the previous sections we showed Tm9 has variable response properties and wide ON receptive fields. To understand how specific to Tm9 are this variability and wide field responses, we recorded another neuron simultaneously. We chose Tm4, because it shares the computational context of Tm9: it is in the OFF-motion pathway and connects to T5 neurons in the lobula. Also, the Tm4 axon terminals in the lobula do not overlap with Tm9 axon terminals (fig. 2.13 A-B). This reduces the cross-talk of the fluorescence signals from both neurons. To image both neurons simultaneously, we used two genetically-encoded calcium indicators with different spectral sensitivity. We used GCaMP6f (green-emitting, T.-W. Chen et al., 2013) in Tm9 neurons, and jRGECO1a (red-emitting, Dana et al., 2016) in Tm4 neurons (fig. $2.13 \mathrm{~B}$ ).

We recorded responses of Tm9 and Tm4 to ON-OFF full-field flashes, and ON and OFF bars. For full-field flashes, both neurons responded preferentially to OFF flashes (fig. 2.13 C-F). Responses of Tm4 were more transient than Tm9 responses. Also Tm4 responded similarly to both OFF transitions: gray to OFF and ON to gray. Tm9 responded with higher amplitude to gray to OFF than ON to gray.

We next analyzed the responses to flashing bars, to get receptive fields from Tm4 and Tm9. Both neuron types responded positively to OFF bars, and with similar spatial extent. However, unlike Tm9, Tm4 neurons did not respond to ON bars. To visualize the average receptive field, we aligned individual tuning curves by maximizing their cross-correlation to a reference tuning curve, which was the average of tuning curves closer to the center of the screen. Again, we saw similarities in the OFF receptive fields of both neurons, but Tm4 average ON tuning curves were flat (fig. 2.14). On the other hand, Tm9 average ON tuning curves for both orientations looked wider than the corresponding average OFF tuning curves. As before, we quantified the receptive field size by fitting a Gaussian and computing its full width at half maximum. We compared the receptive field size distribution for OFF Tm4, OFF Tm9, and ON Tm9 receptive fields. Because the neurons were analyzed independently for all stimuli, pairwise comparisons across stimuli were not possible. The FWHM of OFF receptive fields of Tm4 and Tm9 ranged from about $5^{\circ}$ to $20^{\circ}$, and the FWHM of ON receptive fields of Tm9 ranged from about $10^{\circ}$ to $30^{\circ}$ (fig. 2.15 ). On average FWHM were $11.20^{\circ}$ for Tm4 OFF, $9.80^{\circ}$ for Tm9 OFF, and $19.40^{\circ}$ for Tm9 ON receptive fields. Tm4 had slightly 
Table 2.1: Statistics for $\mathrm{Tm} 9$ receptive field size for $\mathrm{ON}, \mathrm{OFF}$, or noise bars.

Shown are p-values from two-sample two-sided permutation test of mean differences between pairs of stimuli.

\begin{tabular}{lcc}
\hline & Noise - OFF Noise - ON \\
\hline FWHM $\left(^{\circ}\right)$ & & \\
p-value & $6 \cdot 10^{-4}$ & $2 \cdot 10^{-3}$ \\
Mean difference & 4.11 & -5.70
\end{tabular}

larger OFF receptive fields ( $\mathrm{p}$-value $\left.=3 \cdot 10^{-2}\right)^{5}$. Tm9 ON receptive fields were almost twice as large as OFF receptive fields from either neuron type (both p-values equal $1 \cdot 10^{-4}$ ). We also quantified the variability of the distributions, the variance of the Tm9 ON FWHM distribution was higher than the Tm4 OFF FWHM distribution, but the coefficient of variation was smaller for Tm9 ON than for Tm4 OFF and Tm9 OFF. Therefore, wide-field responses are specific to $\mathrm{Tm} 9 \mathrm{ON}$ receptive field, and contribute to the functional variability of Tm9 in terms of variance.

\footnotetext{
${ }^{5}$ Permutation tests, all comparisons were significant under the Holm-Bonferroni method for multiple testing correction.
} 
A

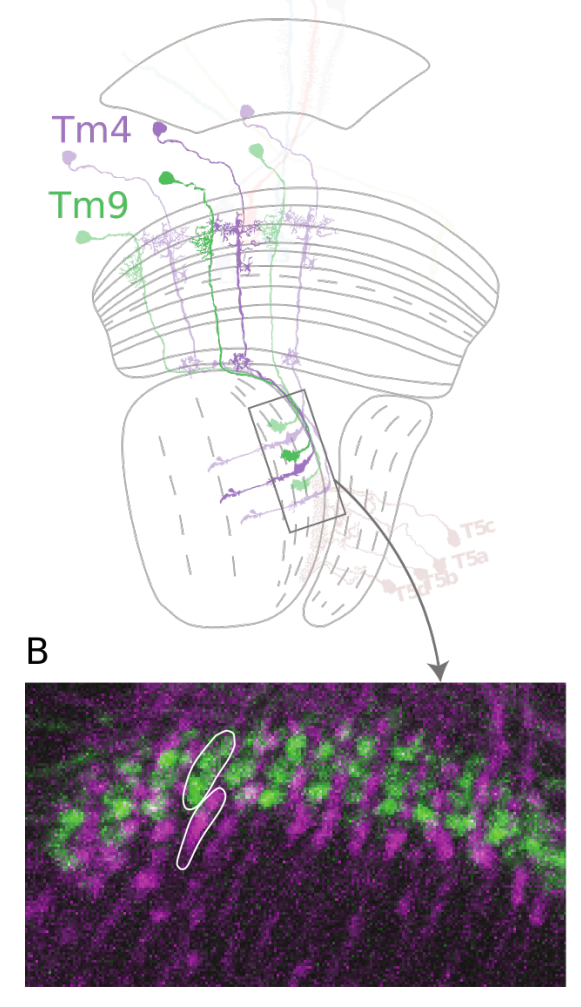

C

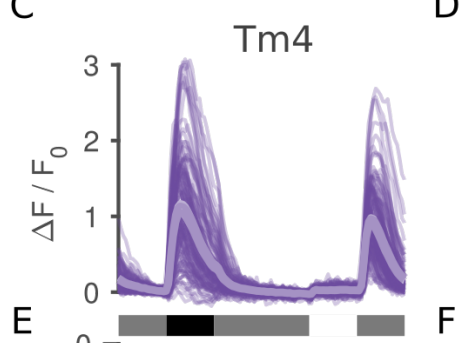

$\mathrm{E}$

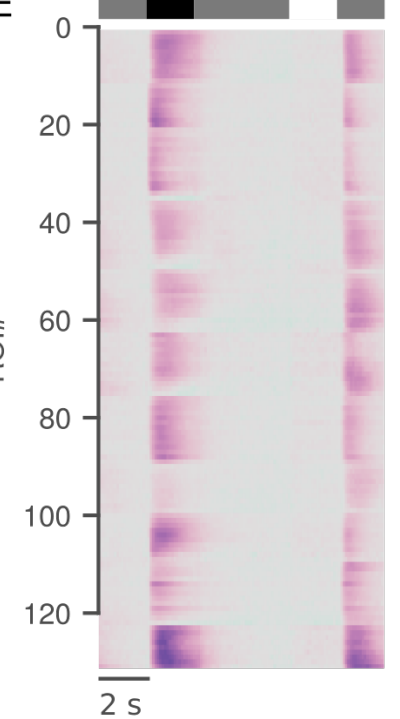

D
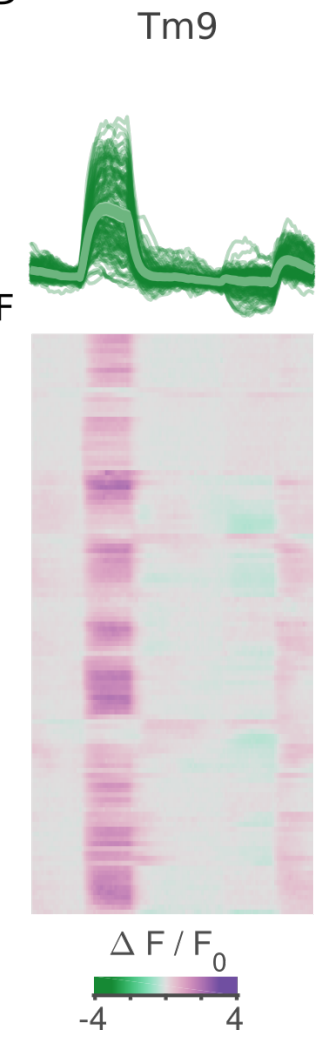

Figure 2.13: Responses to ON-OFF full-field flashes of Tm9 and Tm4 measured simultaneously.

Tm9 calcium responses were measured with GCaMP6f, and Tm4 responses were measured with jRGECO1a (Dana et al., 2016). A. Schematic of the fly visual system including Tm9 in green, and Tm4 in purple, highlighting the location of their axon terminals in the lobula. The rectangle shows imaging region. B. An example imaging region in the lobula with Tm9 axon terminals in green, and Tm4 axon terminals in purple. C. ON-OFF full-field flashes responses of all recorded Tm4 neurons. D. Similar as (C) but for Tm9. E and F. Same data as (C) and (D) but highlighting single neuron responses, color map shows positive responses in purple and negative responses in green. 


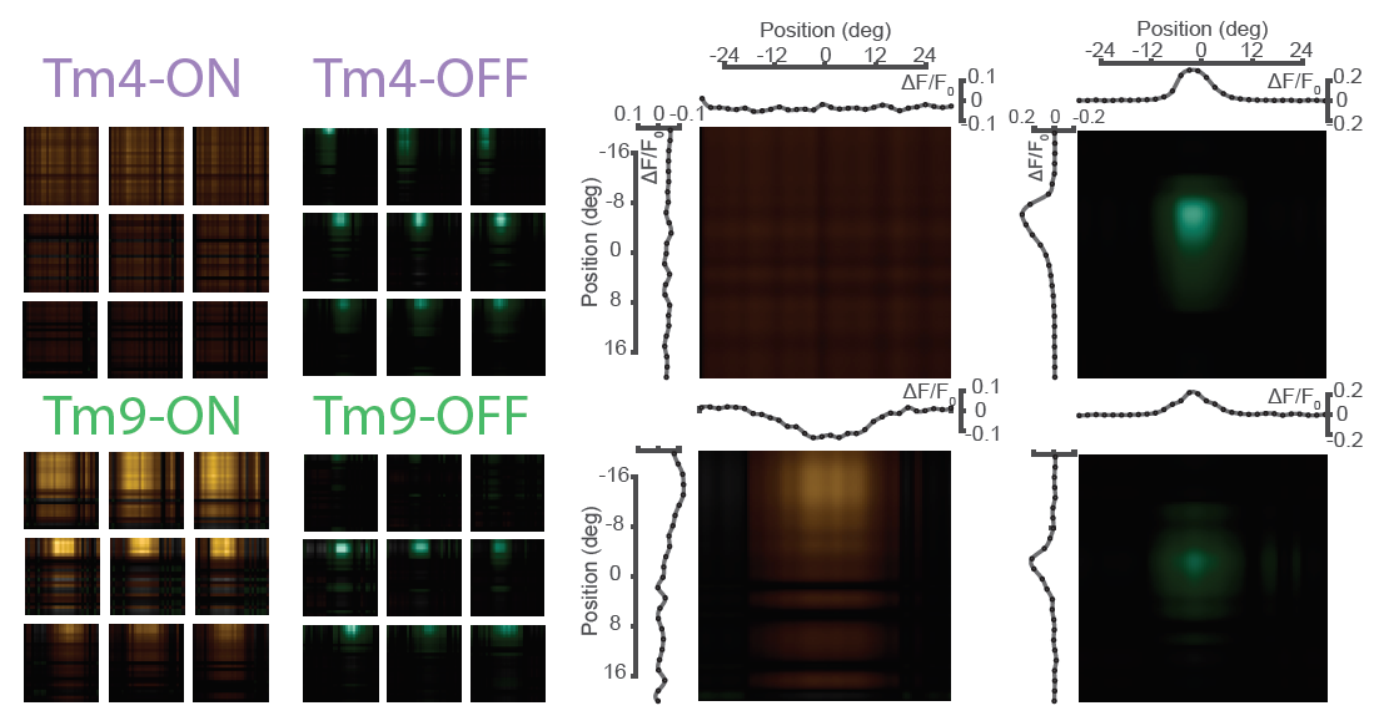

Figure 2.14: Receptive fields of Tm9 and Tm4 measured simultaneously.

Tm4 and Tm9 example receptive fields to ON and OFF bars. Receptive field average after alignment by maximizing their cross-correlation. Tm4 and Tm9 have similar OFF receptive field shapes. There was a bias to record receptive fields in the upper portion of the screen, because the signal was too low in deeper layers looking closer to the screen center.

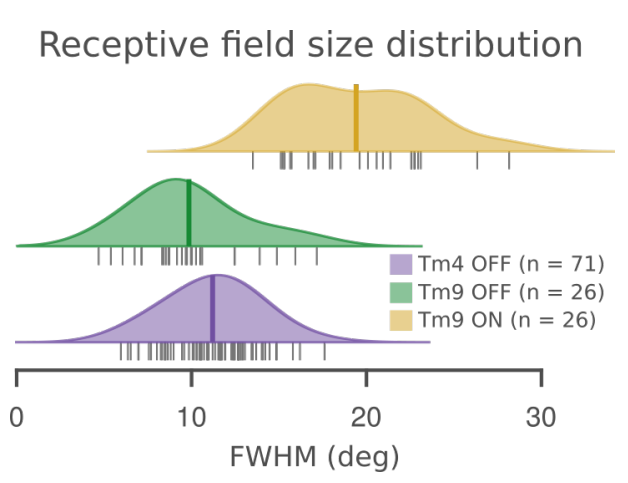

Figure 2.15: Receptive field widths of Tm9 and Tm4 measured simultaneously.

Distribution of receptive field widths, measured as the full width at half maximum from a Gaussian fit. Only Tm9 responded to ON bars. 


\subsection{Receptive fields of the main inputs to the OFF direction selective neurons}

The previous section demonstrated Tm9 but not Tm4 neurons respond to ON stimuli, when measured under exactly the same biological, stimulus, and imaging conditions. We next asked whether other neuron types would exhibit variable receptive fields. We chose to measure the receptive fields of the main input neurons to the T5 direction-selective neurons, namely, $\mathrm{Tm} 1$, $\mathrm{Tm} 2$, and Tm4, in addition to Tm9. There are reports of their receptive fields to OFF bars (Serbe et al., 2016) and to white-noise bars (Arenz et al., 2017). However, given the discrepancy in the literature regarding receptive field properties of $\mathrm{Tm} 9$, we measured these again to reproduce existing results or to get new insights.

We started by characterizing responses to ON-OFF full-field flashes. We recorded neurons that responded to a small $10^{\circ}$ circle flashing in the center of the screen. The responses to the search stimulus were not recorded. The response to small circles did not imply a (strong) response to full-field flashes (fig. 2.16). In fact, the responses of some Tm1 neurons were particularly low (fig. 2.16 A, E). All neuron types responded positively to OFF. Tm9 responded to ON positively or negatively but with small amplitude (fig. $2.16 \mathrm{D}, \mathrm{H}$ ). Some Tm1 neurons responded negatively to the OFF to gray transition. Contrarily, ON responses of Tm2 and Tm4 were not prominent (fig. 2.16 B, C, F, G). All neurons types but Tm9 responded similarly to both OFF transitions: gray to OFF, and ON to gray. Therefore, they seemed to encode the contrast regardless of baseline luminance. About the temporal properties, Tm1 neurons were some transient and some sustained, the sustained ones dominated the average. Tm2 neurons peaked early and decayed to a plateau. Tm4 neurons were transient and decayed back to baseline before the OFF stimulus ended. Tm9 neurons were mostly sustained with some transient exceptions (same data as in fig. 2.7). Therefore, all Tm neurons encoded OFF stimuli with different kinetics, but only Tm9 and Tm1 showed signs of variability.

We next recorded responses to ON and OFF bars, and extracted receptive fields that were fitted by a single Gaussian. Similarly to full-field flashes, all neurons responded positively to OFF bars (fig. 2.17 A-D). Only $\mathrm{Tm} 2$ and Tm9 responded to ON bars, and negatively so (fig. $2.17 \mathrm{~B}, \mathrm{D}$, except for 3 out of $90 \mathrm{Tm} 1$ neurons in A). We quantified the FWHM of the Gaussians as a measure of receptive field size. All neurons had very similar narrow (less than $10^{\circ}$ ) receptive fields for OFF stimuli (fig. 2.17 E). Tm2 and $\mathrm{Tm} 9 \mathrm{ON}$ receptive fields were almost twice as wide as OFF receptive 
fields, and had a larger variance of the FWHM distribution (fig. 2.17 E, G). Interestingly, no receptive field was wider than $40^{\circ}$, contrasting with data of previous section. Thus, Tm9 properties on this data set were less variable. As comparison, we overlaid the quantification of the coefficient of variation of the first dataset on (fig. 2.17 F). The lack of Tm9 wide receptive fields can stem from diverse sources, and will be discussed at the end of the thesis. Nevertheless, this data confirmed the narrow OFF receptive fields reported in (Serbe et al., 2016), but additionally demonstrated that not only Tm 9 but also Tm 1 can encode ON stimuli over wider regions than covered by one eye column, and do so with different kinetics. Furthermore, Tm9 was the only neuron showing variability at both the spatial and temporal response properties. 


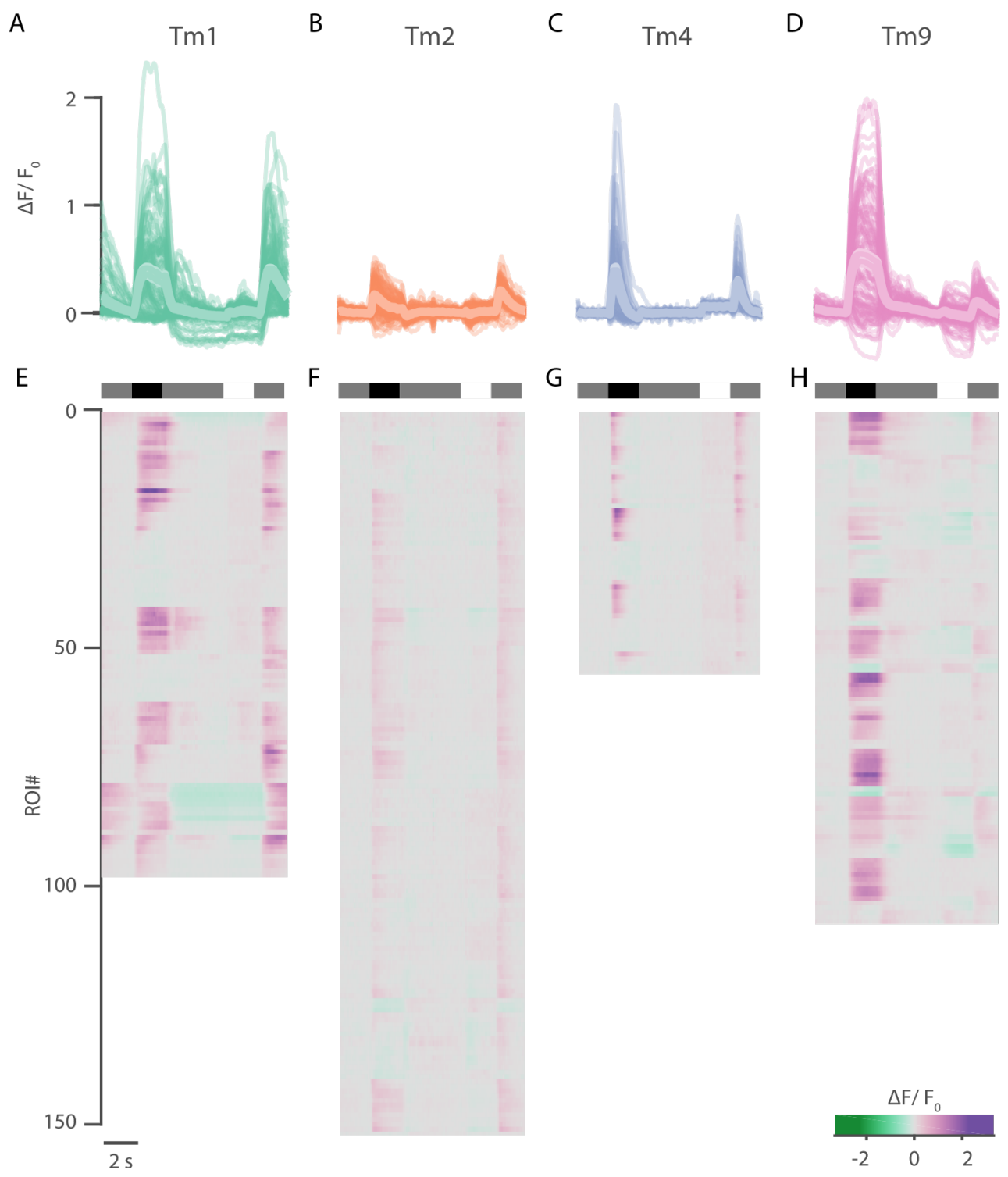

Figure 2.16: Responses of OFF-pathway neurons to ON-OFF full-field flashes.

Shown trial averaged responses per neuron to a sequence of $2 \mathrm{~s}$ gray, $2 \mathrm{~s}$ OFF, $4 \mathrm{~s}$ gray, and $2 \mathrm{~s}$ ON. A-D, single neuron responses (thin lines), on top is the mean with the standard error across neurons, for Tm1, Tm2, Tm4 and Tm9, respectively. E-H. Single traces from A-D, respectively, stacked on top of each other for better visualization of response variability. 


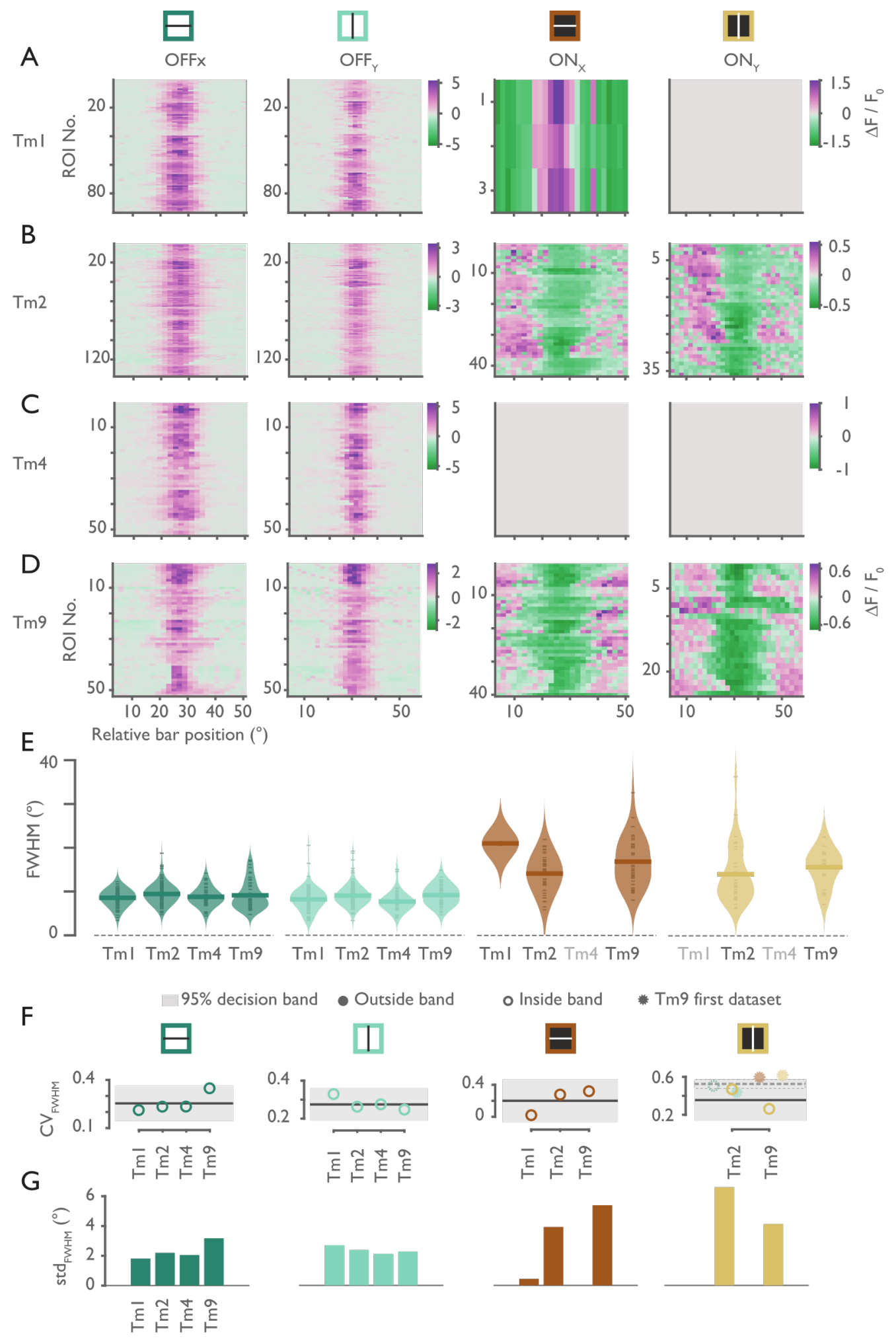


Figure 2.17: Receptive fields of the main inputs to the OFF pathway. Receptive fields for horizontal and vertical ON and OFF bars for (A) Tm1, (B) Tm2, (C) Tm4 and (D) Tm9. Receptive field are aligned by crosscorrelation to receptive fields close to the center of the screen, OFF receptive field shift is applied to the respective ON receptive fields. E. Receptive field width distributions of Tm1, Tm2, Tm4, and Tm9. Widths are the FWHM from single Gaussian fits to receptive fields in (A-D). F. Coefficient of variation from distributions in E. Line is the average coefficient of variation for all conditions, and the band shows the 95\% bootstrapped confidence interval, open and closed circles indicate values inside and outside the band, respectively. Overlaid is the quantification of previous Tm9 dataset (fig. 2.6). G. Standard deviation of distributions in E. 



\section{Mechanisms composing and tuning the receptive field of Tm9}

In the previous chapter demonstrated Tm9 functional properties can be variable. In this chapter, we delve deeper into the mechanisms making up the receptive field of Tm9. The first section sketches the presynaptic circuitry of Tm9, including new types of wide-field neurons. In the second section we probe the role of single cell types in the receptive fields of Tm9 neurons. Finally, in the third section, we extend the study of Tm9 receptive field mechanisms to electrical coupling via gap junctions to Tm9.

\subsection{Sketching the presynaptic circuitry of $\operatorname{Tm} 9$ neurons}

The receptive field of a neuron is a macroscopic description of the neuronal response properties arising from the integration of inputs by the neuron. Sensory neurons can transduce many modalities of physical stimuli into electrical and chemical signals that are transmitted to downstream neurons. Thus, inputs to a non-sensory neuron are mainly chemical or electrical. Chemical substances can activate receptors in the neuron that activate signaling cascades with a myriad of effects. These substances can be divided into neuromodulators and neurotransmitters. Neurotransmitters are substances released by a neuron that activate a specific target neuron. The pair of releasing-receiving portions of neurons is called a chemical synapse. On the other hand, neuromodulators are substances that regulate the properties of a set of neurons with longer lasting effects than those of chemical synapses. Electrical synapses connect two neurons via gap junctions that connect their cytosol allowing transmission of ions and small signaling molecules. Here we focus on how the chemical and electrical 
synapses onto $\mathrm{Tm} 9$ shape its receptive field.

\section{Anatomical mapping}

We first looked for the neuron types with chemical synapses onto Tm9. We used anatomical knowledge of neuron types and connectomics.

\section{Connectomics}

First, from connectomes of the Drosophila melanogaster visual system (Takemura, Xu, et al., 2015) we identified the core circuitry of Tm9. Tm9 receives inputs from $\mathrm{L} 3, \mathrm{Mi} 4, \mathrm{C} 3, \mathrm{Tm} 20, \mathrm{Tm} 1, \mathrm{C} 2$, and L4. However, data extracted from (Flyem Tables 2017) gave different information ${ }^{1}$. Nevertheless, Tm9 receives input mainly from L3 and other types of columnar neurons.

Connectivity is more distributed for Tm9 than other OFF pathway interneurons Interestingly, while we looked at connectomics data that compared reconstructions of seven medulla columns, we noticed Tm9 had more connectivity variations than other neurons (supplementary tables in Takemura, Xu, et al., 2015). Furthermore Tm9 received fewer input synapses than other OFF pathway neurons, namely Tm 1 and Tm2. Indeed, the major cell type inputs to Tm1 and Tm2 had larger synapse counts than all of the Tm9 inputs together (fig. 3.1 A). Thus, to get the normalized contribution of each cell type, we calculated the proportion of input synapses relative to the total. More than $70 \%$ of the synapses to $\operatorname{Tm} 1$ and Tm2 came from a single cell type, while only about $50 \%$ synapses to Tm 9 were contributed by its main input cell type (fig. $3.1 \mathrm{~A}$ ). Therefore, Tm9 integrates input more uniformly across cell types, than its OFF pathway counterpart neurons Tm1 and Tm2.

\section{Fluorescence reconstitution across synaptic partners}

Tm9 receives inputs from many columnar cell types, but the wide-field responses of Tm9 suggested wide-field neurons could contribute to Tm9 receptive fields. Because the neurites of wide-field neurons span multiple columns, they could not be entirely reconstructed to be mapped to a cell type, and thus were missing from published connectomes (Takemura, Bharioke, et al., 2013; Takemura, Xu, et al., 2015).

\footnotetext{
${ }^{1}$ Website is no longer available/accessible.
} 

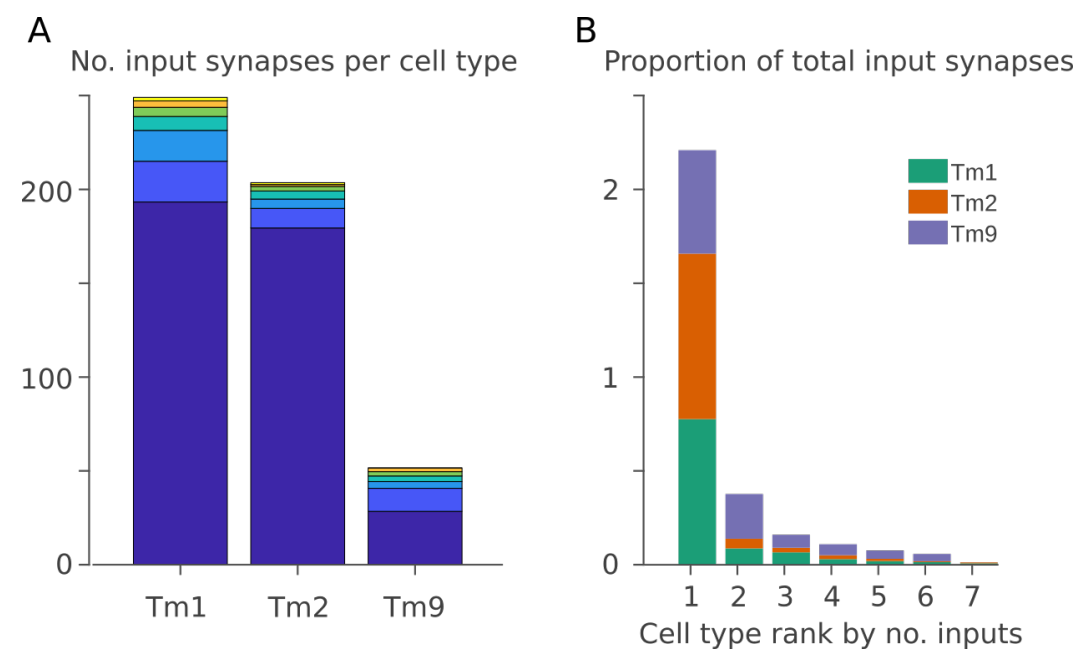

Figure 3.1: Input synapse distribution of the major OFF motion pathway interneurons.

Input synapse distribution to $\mathrm{Tm} 1$, Tm2, and $\mathrm{Tm} 9$, from data in (Takemura, $\mathrm{Xu}$, et al., 2015). A. Stacked bar plot of total number of input synapses, each color indicates a different cell type, colors are independently assigned for Tm1, Tm2, and Tm9. B. Distribution of synapses per cell type. Each bar represents the proportion of input synapses contributed by each cell type relative to the total count in (A), cell types were ranked accordingly. $\mathrm{Tm} 1$ and $\mathrm{Tm} 2$ receive more than half their inputs from a single cell type. Tm9 inputs distribute more uniformly across cell types than Tm1 and Tm2 inputs.

To identify possible wide-field inputs into Tm9 we used a light microscopy characterization of wide-field neurons (Nern, Pfeiffer, and Rubin, 2015). We shortlisted candidates based on the arborization size and position within the medulla. Tm9 dendrites arborize in the medulla layer 3 (M3). Wide-field neurons in this location are called distal medulla (Dm) neurons. The candidate neurons arborizing over tens of columns in layer M3 were Dm4, Dm12, and Dm20 (fig. 3.2 A). Also Dm9 arborizes in this layer, but because it also projects to more proximal layers we did not consider it in our initial screening. However, we collected preliminary data on Dm9 effects into Tm9 receptive fields (section 3.2).

To test for synaptic contact we used GRASP (genetic reconstitution across synaptic partners) (Feinberg et al., 2008; Macpherson et al., 2015). In this fluorescence reconstitution assay, the potential postsynaptic neuron expressed one portion of the GFP molecule on its membrane, and the potential presynaptic neuron expressed the complementary part of the 


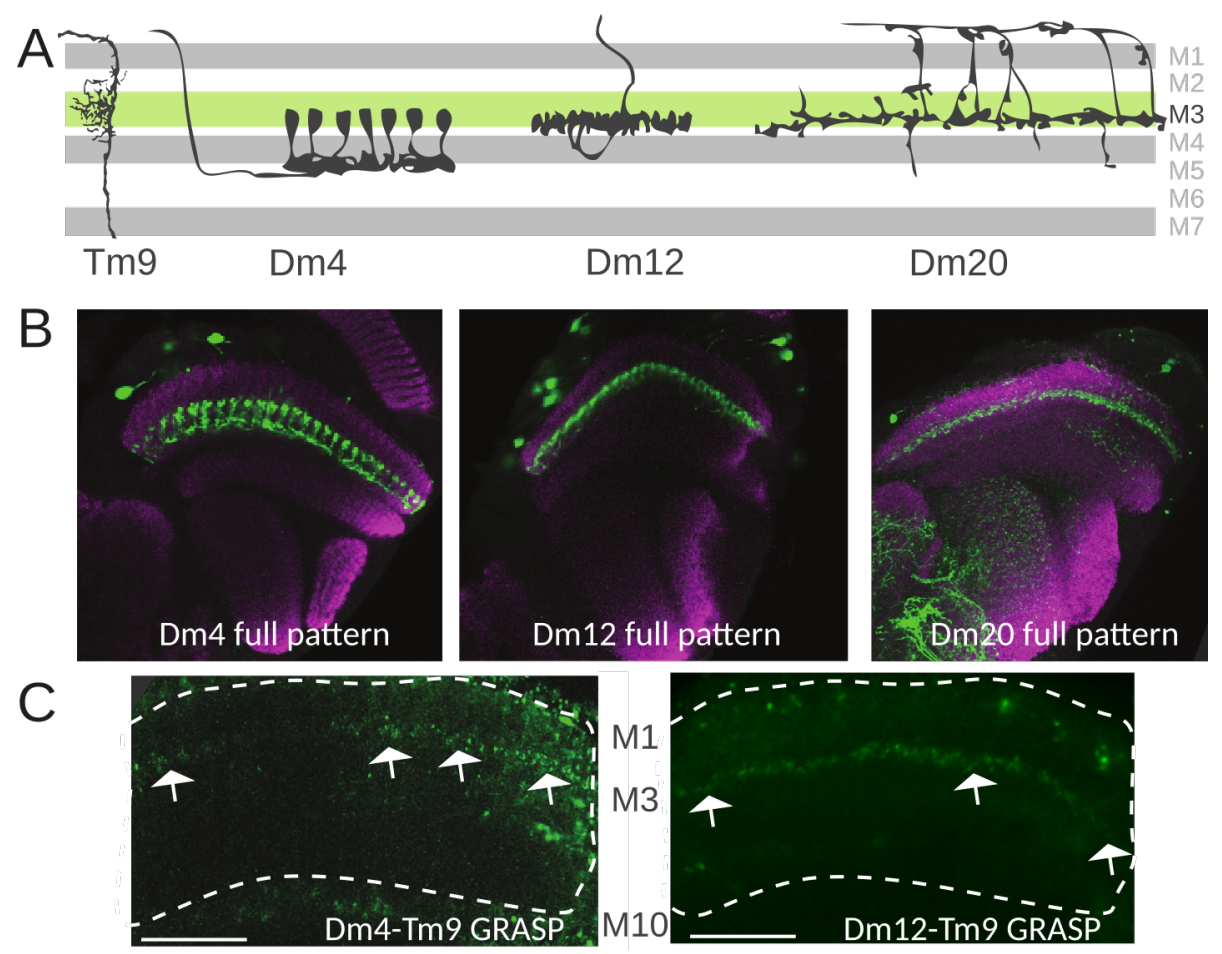

Figure 3.2: Dm4 and Dm12 synapse onto Tm9: GRASP assay.

GRASP (GFP reconstitution across synaptic partners) assay from Dm4, Dm20, and Dm20 to Tm9. A. Schematic of neuron types that arborize in layer M3 (after (Nern, Pfeiffer, and Rubin, 2015)). B. Full expresion patterns of genetic lines for Dm4, Dm12, and Dm20 neurons. C. GRASP signals from Dm4 to Tm9, and Dm12 to Tm9, indicating Tm9 receives wide-field inputs.

GFP molecule attached to a synaptic vesicle protein (synaptobrevin). Thus, when vesicles are released upon exocytosis, the vesicle membrane fuses with the cell membrane, and the GFP tagged synaptobrevin faces the extracellular medium. If both neurons are in proximity, the portions of the GFP molecule interact and complement each other, resulting in a fluorescence signal. Therefore, GRASP fluoresce signals are a proxy of synaptic connectivity, one cell membrane close to the presynaptic release sites of other cell.

For GRASP experiments, we imaged the endogenous fluorescence of the brains immediately after dissection with no extra treatments. We used genetic lines for Dm4, Dm12, Dm20 that were very specific, except Dm20 line, which was nevertheless specific in the M3 layer (fig. 3.2 B). We performed GRASP using Tm9 as postsynaptic neuron, and Dm4, Dm12, 
and Dm20 as presynaptic neurons. We observed GRASP signals in Tm9Dm4, and Tm9-Dm12 (fig. 3.2 C). Therefore, Dm4 and Dm12 are potential wide-field inputs to Tm9.

\section{Synaptic site distribution in wide-field neurons}

To understand the input-output relation in Dm neurons, we stained them with markers for the somatodendritic compartment (DenMark (Nicolaï et al., 2010)), and for synaptic vesicles (synaptotagmin fused to GFP Zhang, Rodesch, and Broadie, 2002). Dm12 had a uniform distribution for both pre- and postsynaptic sites (fig. 3.3 D, F). Contrarily, Dm4 showed a patchy presynaptic site distribution (fig. 3.3 C, E). Moreover the presynaptic release sites localized to the layer M3 bulb-like projections of Dm4 (data not shown). This suggested Dm4 is a substrate for circuit variability in the fly eye.

We investigated further Dm4 variable presynaptic distribution. To test whether the patchy presynaptic distribution of Dm4 depended on the marker, we used different markers to label the presynaptic release sites. To check the influence of the fluorescent protein fused to synaptotagmin, we used RFP in addition to GFP fused synaptotagmin. We also used a different marker fused with a different fluorescent protein, namely the active zone marker Bruchpilot fused to mCherry. The coverage of the distribution varied from only one neuron lacking presynaptic sites (fig. $3.4 \mathrm{~A}$ ), to only couple neurons having presynaptic sites (fig. 3.4 C). We concluded, despite possible effects of the presynaptic marker and fused fluorescent protein, that Dm4 has a non-uniform presynaptic site distribution. Because Tm9 gets inputs from Dm4 and Dm4 has a heterogeneous presynaptic distribution it is important to study the functional consequences. Thus, we next studied the functional connectivity of Dm4 and Tm9, among other neuron types. 
A

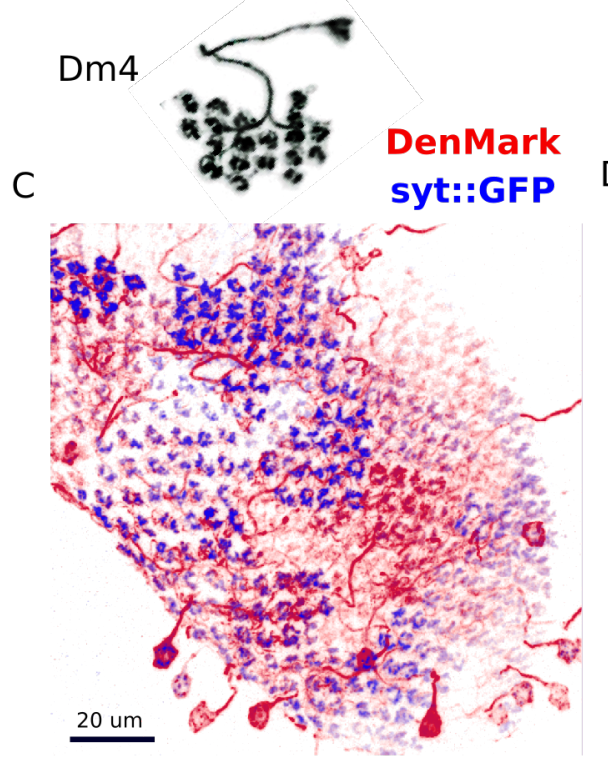

E

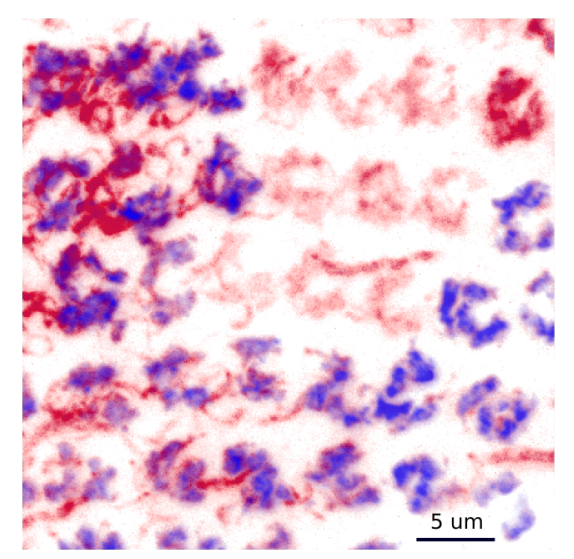

B

$\operatorname{Dm} 12$
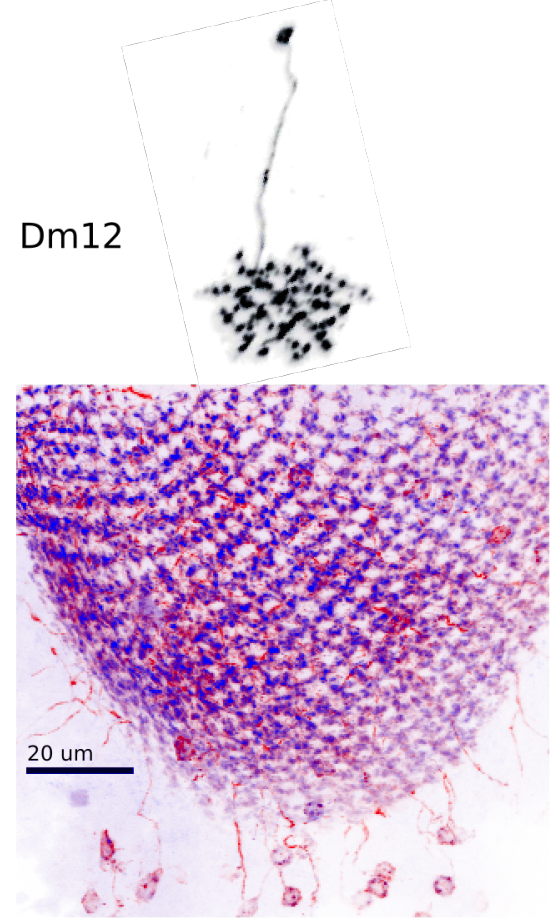

$\mathrm{F}$

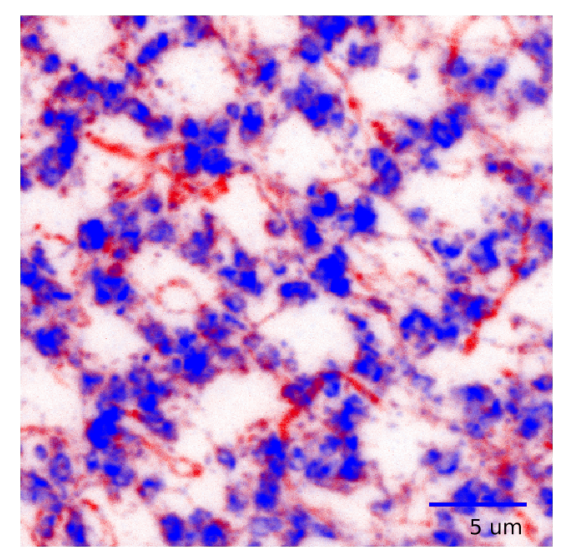

Figure 3.3: Dm4 presynaptic site distribution is non-uniform.

Dm4 and Dm12 postsynaptic sites (soma and dendrites) labeled with DenMark in red, and presynaptic release sites labeled with synaptotagmin fused with GFP (syt::GFP) in blue. Shown are maximum intensity projections of confocal microscopy stacks of example optic lobes after immunostaining to amplify the signal. A-B. Example Dm4 (A) and Dm12 (B) neurons adapted from (Nern, Pfeiffer, and Rubin, 2015). C-D. Full optic lobe image of Dm4 (C) and Dm12 (D). Only some Dm4 neurons lacked presynaptic sites. E-F. Zoomed version of (C, D). It is clear Dm4 neurons expressed DenMark overall, but syt::GFP was missing in some neurons like the one in (E).) 
A Dm4 expressing synaptotagmin::RFP
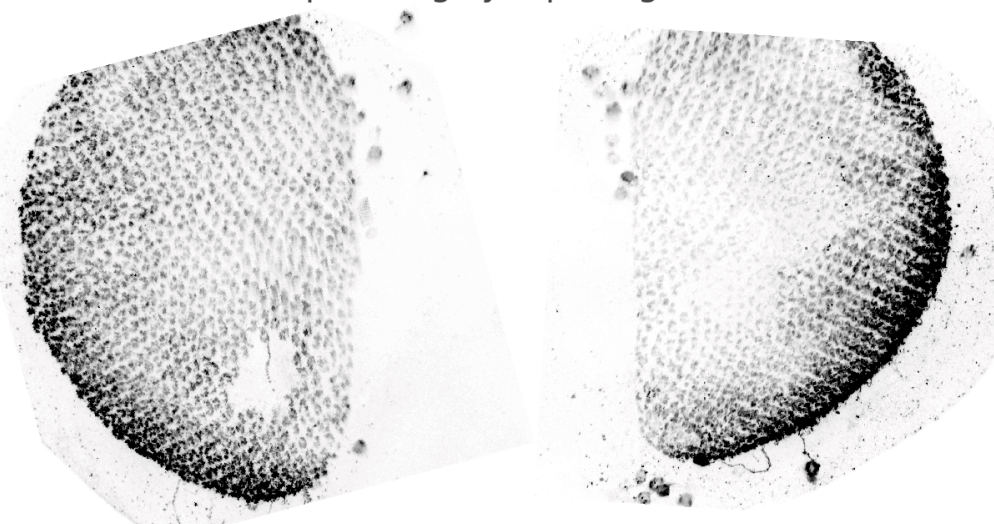

B Dm4 expressing synaptotagmin::GFP
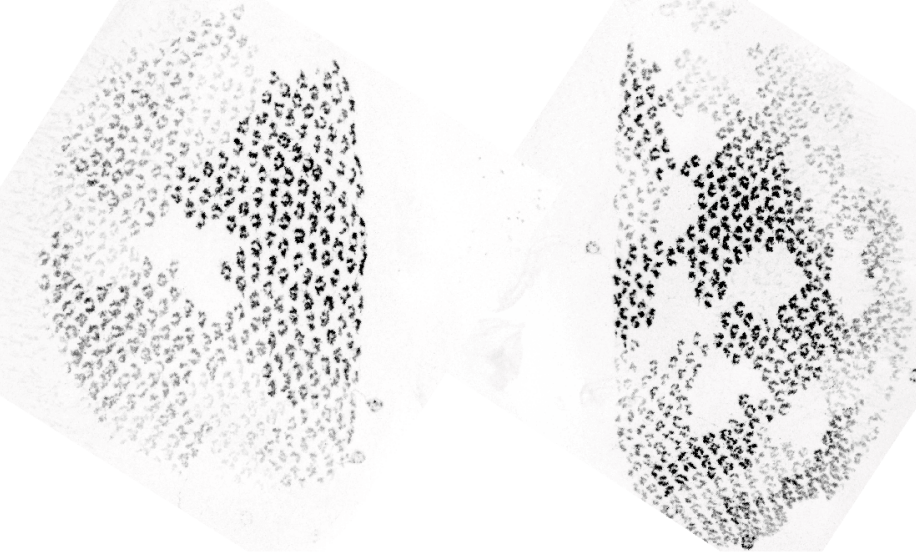

C Dm4 expressing bruchpilot::mCherry
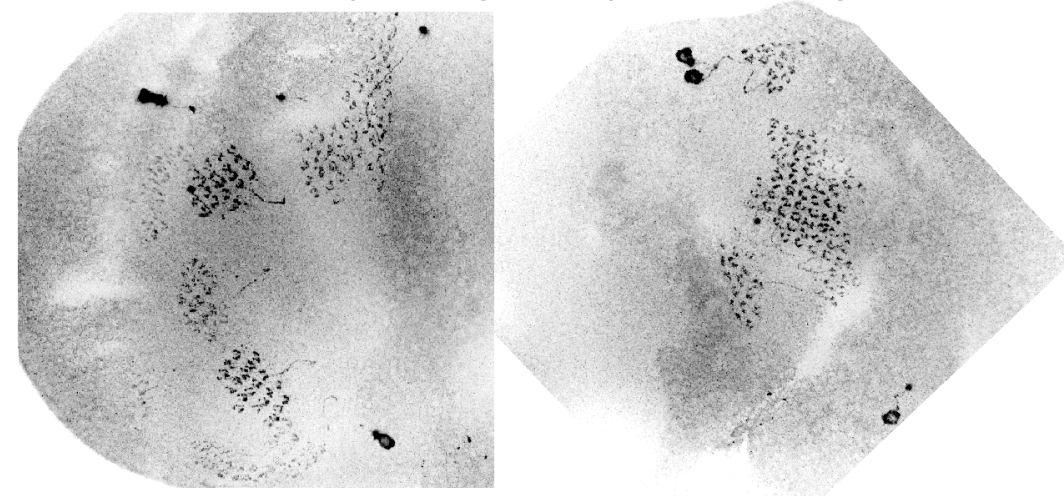

Figure 3.4: Dm4 presynaptic site distribution is non-uniform irrespective of markers.

Dm4 presynaptic release sites from maximum intensity projections of confocal microscopy stacks. Each panel shows the two optic lobes of an example fly. Dm4 expressed different presynaptic markers which were immunostained to amplify the signal. A. Synaptotagmin fussed to RFP: the left optic lobe shows a hole in lower part. B. Synaptotagmin fused to GFP: both optic lobes have several holes. C. Bruchpilot (active zone marker) fused to mCherry: very few neurons were labeled. similar results were obtained for three to five flies. 


\section{Functional mapping: optogenetics}

Purely anatomical connectivity does not tell what is the effect of the activity of a presynaptic neuron on Tm9's activity. Functional connectivity describes this relationship. We can activate one neuron and check the effect on another neuron. Here we use optogenetics together with in vivo twophoton calcium imaging to stimulate one neuron type and record responses of Tm9 neurons, respectively. We used a red-shifted opsin CsChrimson to minimize photoreceptor activation and avoid confounding effects of visual vs optogenetic responses to light. Nevertheless, neurons responded to the red light ( $625 \mathrm{~nm}$ LED, data not shown). Thus, performed the experiments in blind flies (fig. $3.6 \mathrm{w}+$ ). Blind flies were norpA (no receptor potential A) mutants, the norpA gene encodes phosphatidylinositol-specific phospholipase C (PLC) that is required for phototransduction (McKay et al., 1995).

We recorded Tm9 responses to full-field optogenetic stimulation of following cell types that expressed CsChrimson: photoreceptors R1-R6, and R8; lamina neurons L1, L2, and L3; distal medulla neurons Dm4, Dm12, and Dm20; and other columnar neurons like Tm1, Mi9, and C3. For each group we used as a control one cell type without known direct synapses: L1, Mi9, and Dm20. The cell types used are sketched in fig. 3.5

We tested different stimuli, from single light pulses lasting $25 \mathrm{~ms}$ to trains of $5 \mathrm{~ms}$ long pulses at $40 \mathrm{~Hz}$. Response kinetics were qualitatively similar for single pulses and pulse trains (data not shown). We further probed the effect of light power on the responses, again, we did not observe any effects on the kinetics (fig. 3.6). Next, we only show results from pulse train stimulation used for all cell types (fig. 3.7).

Photoreceptor activation led to negative Tm9 responses. All lamina neurons increased calcium in Tm9, while all distal medulla neurons decreased calcium in Tm9. Interestingly, Dm4 also increased calcium in a subset of Tm9 neurons. This duality of Dm4 effects adds to the duality of Dm4 neurons with and without presynaptic sites (fig. 3.4).

Different neuron types elicited different response time courses in Tm9. The short initial negative response evoked by R1-R6 was masked by the stimulus, but the negative response peak of $\operatorname{Tm} 9$ was visible for the longer lasting effects of R8 activation. Interestingly, the positive rebound evoked by R1-R6 was more prominent than the one evoked by R. Tm9 responses evoked by L1 lasted longest, consistent with indirect connections from L1 to Tm9 supported by silencing experiments (Fisher, Leong, et al., 2015). L2 and L3 evoked responses decayed within $10 \mathrm{~s}$. Dm4 and Dm12 wide-field neurons evoked responses that decayed faster than Dm20 evoked responses. 


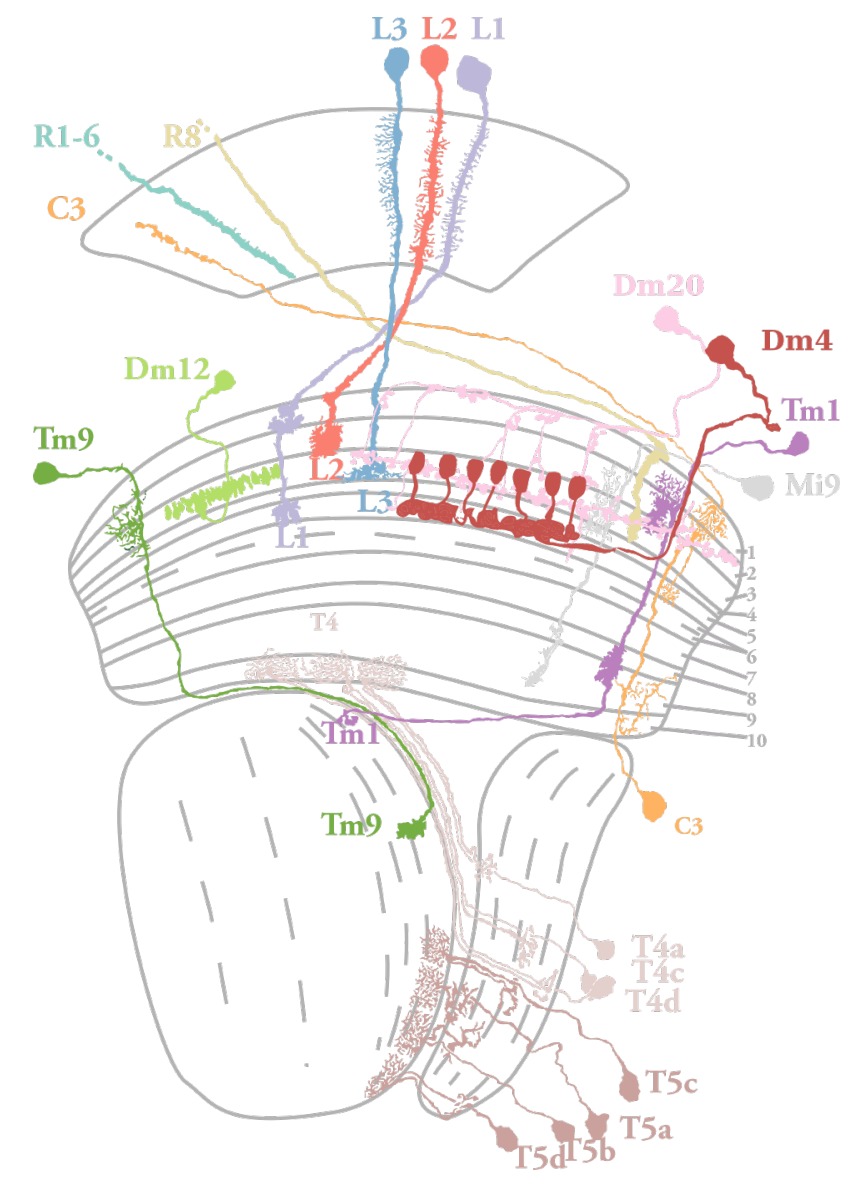

Figure 3.5: Cell types used for optogenetic mapping of Tm9 inputs.

Schematic of the fly optic lobe with cell types overlaid. Anatomy of cell types adapted from (Fischbach and Dittrich, 1989; Nern, Pfeiffer, and Rubin, 2015).

Also both positive and negative Tm9 responses evoked by Dm4 had similar kinetics. From the remaining neurons Mi9 evoked negative responses that decayed fastest (within $5 \mathrm{~s}$ ). Tm 1 evoked responses qualitatively similar to the ones evoked by R1-R6 photoreceptors, a sharp negative peak followed by a positive longer lasting rebound. Finally, responses elicited by C3 were initially negative with small amplitude and then positive with larger amplitude, the whole effect decayed within $10 \mathrm{~s}$.

Activating L3 led to the strongest Tm9 responses, consistent with L3 being the main chemical input to Tm9 (Takemura, Bharioke, et al., 2013), and with silencing experiments of L3 chemical inputs to Tm9 (Fisher, Leong, et al., 2015). Interestingly, all neurons elicited responses in Tm9, 


$\begin{array}{lccc}\text { Stimulus } & 625 \mathrm{~ms} \text { train of } 5 \mathrm{~ms} \text { pulses @ } 40 \mathrm{~Hz} & 25 \mathrm{~ms} \text { pulse } \\ \text { LED power }\left(\mu \mathrm{W} / \mathrm{mm}^{2}\right) & 9.4 & 17.3 & 29.4\end{array}$
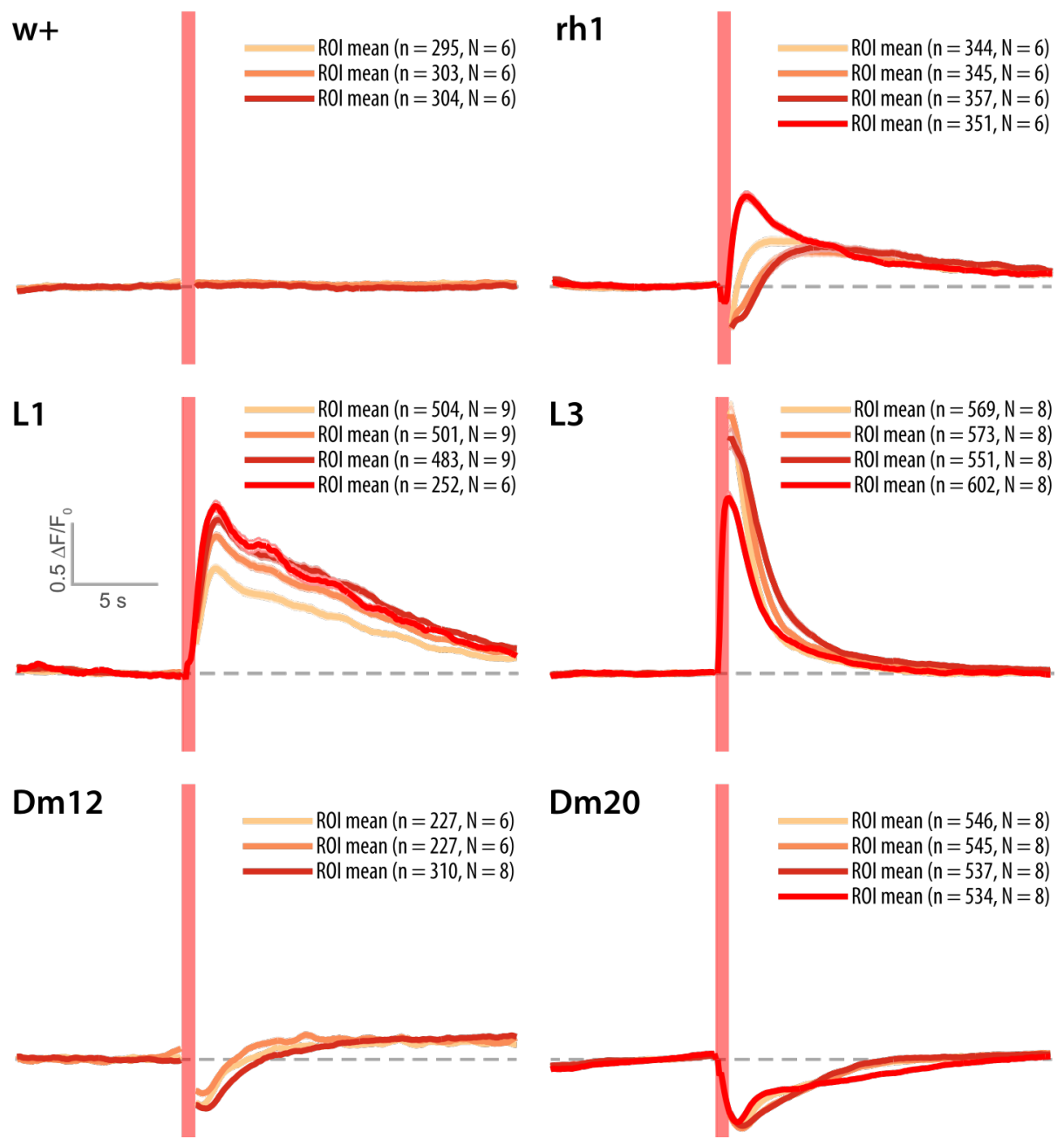

Figure 3.6: Different stimulation powers and durations led to similar Tm9 responses.

Optogenetic activation of cell types using CsChrimson (Klapoetke et al., 2014) while imaging Tm9 expressing GCaMP6f in blind flies. Traces overlaid for different light powers, and for train of pulses vs the shortest pulse attainable. $\mathrm{W}+$ are control blind flies (norpA mutants). 
A

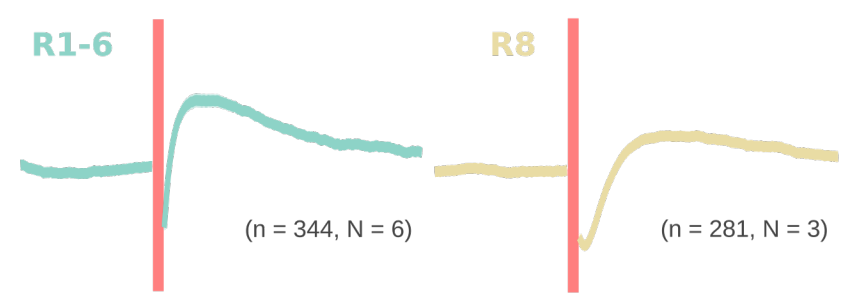

B
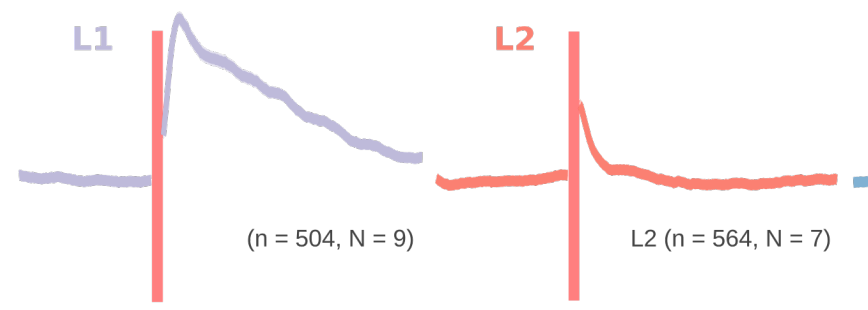

L3

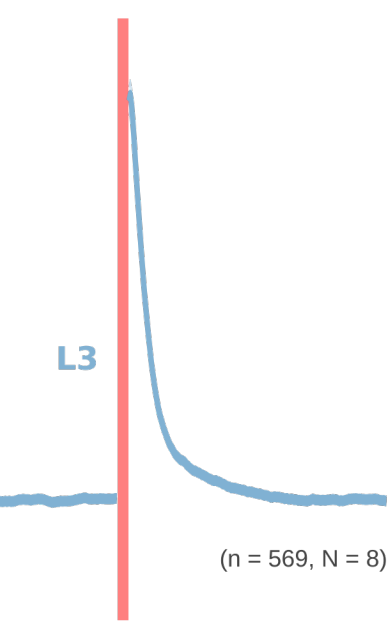

C
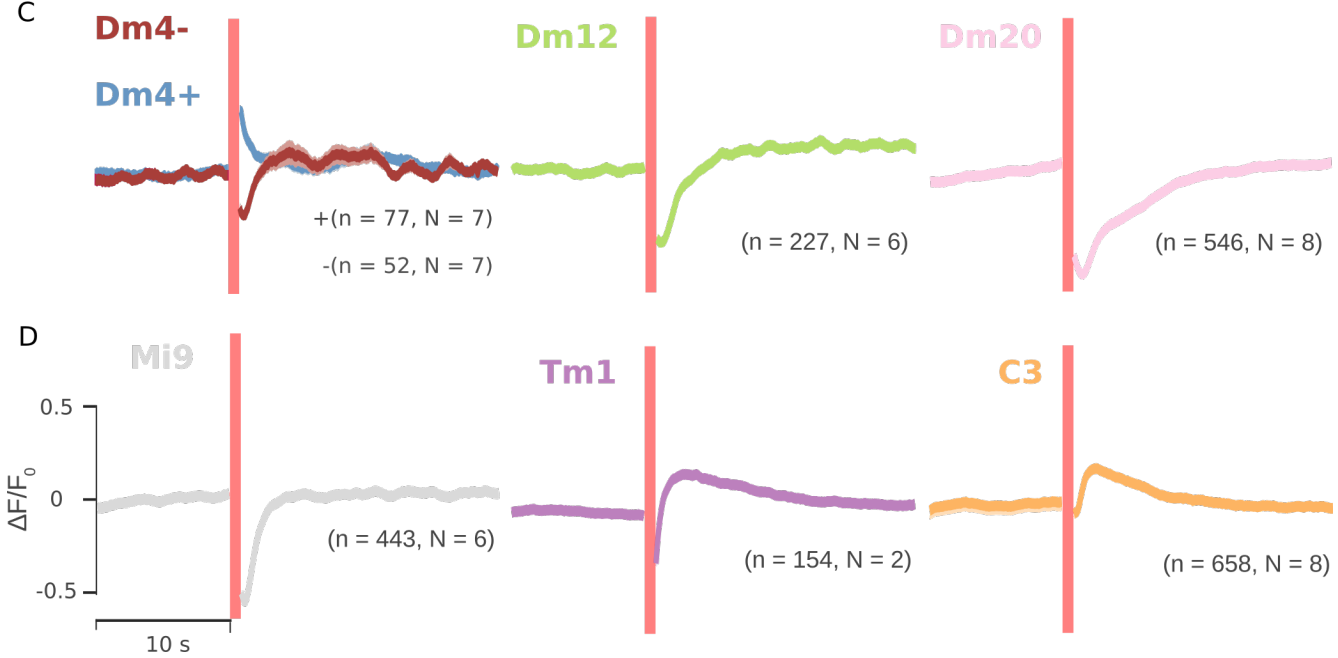

Figure 3.7: Tm9 integrates input from different cell types.

Optogenetic activation of cell types using CsChrimson while imaging Tm9 expressing GCaMP6f in blind flies. A. Photoreceptor inputs. B. Lamina inputs. C. Wide-field inputs. D. Columnar medulla inputs. 
even the ones without direct synapses, such as L1, Mi9, and Dm20 (no observed GRASP). We confirmed functional wide-field inputs into Tm9, namely, Dm4, Dm12 and Dm20. Overall, we showed Tm9 could integrate both direct and indirect inputs of different response polarity and kinetics from diverse cell types (fig. 3.7).

\subsection{Single cell type contribution to the receptive field of $\mathrm{Tm} 9$ neurons}

We confirmed a diverse set of inputs into Tm9, both anatomically and functionally. In this section, we proceeded to test the contribution of several cell types to $\operatorname{Tm} 9$ receptive fields. We did so by recording $\operatorname{Tm} 9$ receptive fields upon genetic silencing of input cell types. We silenced input neurons by overexpression of the inward-rectifying potassium channel Kir2.1 that hyperpolarized their membrane potential (Baines et al., 2001). We recorded responses of Tm9 neurons to ON-OFF full-field flashes and ON and OFF bars (like in sec. 2.1).

Table 3.1: Statistics for Tm9 ON-OFF full-field flashes response properties under block of lamina neurons L1, L2, or L3.

Shown are p-values from two-sample two-sided permutation test of mean differences between control and the condition mentioned. Mean difference is control minus L1-, L2-, or L3-input block.

\begin{tabular}{|c|c|c|c|c|c|c|}
\hline & \multicolumn{2}{|c|}{ L1 block } & \multicolumn{2}{|c|}{ L2 block } & \multicolumn{2}{|c|}{ L3 block } \\
\hline & $\mathrm{p}$-value & Mean diff & p-value & Mean diff & p-value & Mean diff \\
\hline Mean OFF $\left(\frac{\Delta F}{F_{0}}\right)$ & $4.60 \cdot 10^{-1}$ & $-3.40 \cdot 10^{-2}$ & $9 \cdot 10^{-1}$ & $-5.80 \cdot 10^{-3}$ & $9.30 \cdot 10^{-1}$ & $-4 \cdot 10^{-3}$ \\
\hline Mean ON $\left(\frac{\Delta F}{F_{0}}\right)$ & $2 \cdot 10^{-5}$ & $9.30 \cdot 10^{-2}$ & $4.60 \cdot 10^{-2}$ & $2.80 \cdot 10^{-2}$ & $5.40 \cdot 10^{-1}$ & $-7.70 \cdot 10^{-3}$ \\
\hline Polarity index & $4.30 \cdot 10^{-1}$ & $8.60 \cdot 10^{-2}$ & $6.70 \cdot 10^{-1}$ & $-4.80 \cdot 10^{-2}$ & $4.60 \cdot 10^{-1}$ & $-7.70 \cdot 10^{-2}$ \\
\hline Sustenance index & $3.70 \cdot 10^{-1}$ & $-3.90 \cdot 10^{-2}$ & $8.20 \cdot 10^{-1}$ & $9 \cdot 10^{-3}$ & $6.70 \cdot 10^{-2}$ & $8.10 \cdot 10^{-2}$ \\
\hline Half-rise time (s) & $4.30 \cdot 10^{-1}$ & $-3 \cdot 10^{-2}$ & $8.90 \cdot 10^{-1}$ & $-5 \cdot 10^{-3}$ & $1.70 \cdot 10^{-2}$ & $8 \cdot 10^{-2}$ \\
\hline Time to extreme (s) & $1.90 \cdot 10^{-2}$ & $-2.30 \cdot 10^{-1}$ & $9.50 \cdot 10^{-1}$ & $5.70 \cdot 10^{-3}$ & $3 \cdot 10^{-1}$ & $1 \cdot 10^{-1}$ \\
\hline Response quality & $4.20 \cdot 10^{-3}$ & $-6.10 \cdot 10^{-2}$ & $2.50 \cdot 10^{-2}$ & $4.60 \cdot 10^{-2}$ & $2.60 \cdot 10^{-4}$ & $8.20 \cdot 10^{-2}$ \\
\hline
\end{tabular}




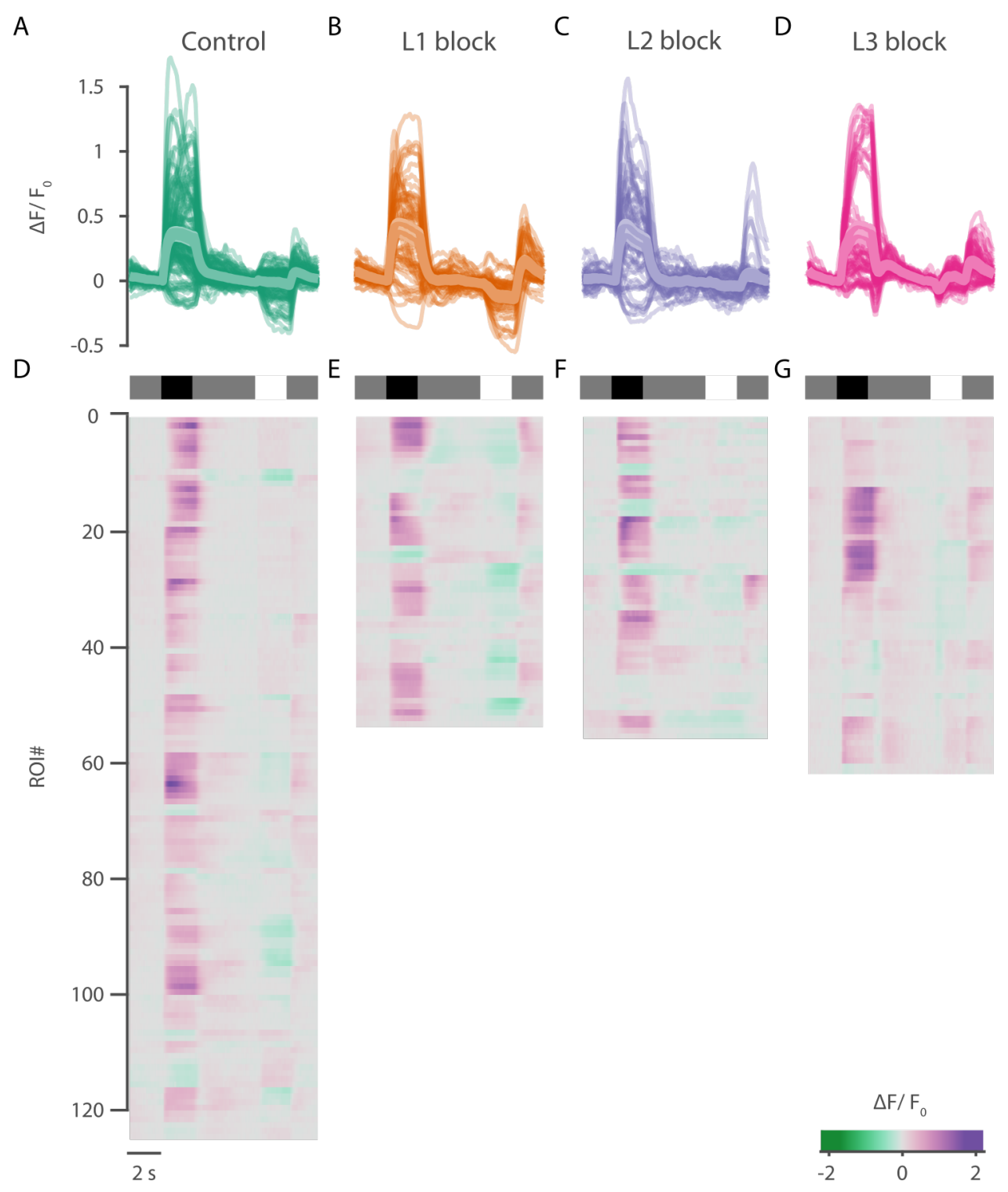

Figure 3.8: Tm9 responses to ON-OFF full-field flashes without input from lamina neurons L1, L2, or L3.

A-D. Trial averaged responses to a sequence of gray-OFF-gray-ON, of $4 \mathrm{~s}$, $2 \mathrm{~s}, 4 \mathrm{~s}$ and $2 \mathrm{~s}$ duration, respectively. Thin lines indicate single neurons, on top is the mean and standard error. Controls (A), L1 (B), L2 (C), and L3 (D) block. E-H. Traces in A-D stacked for better visualization of different response kinetics. 

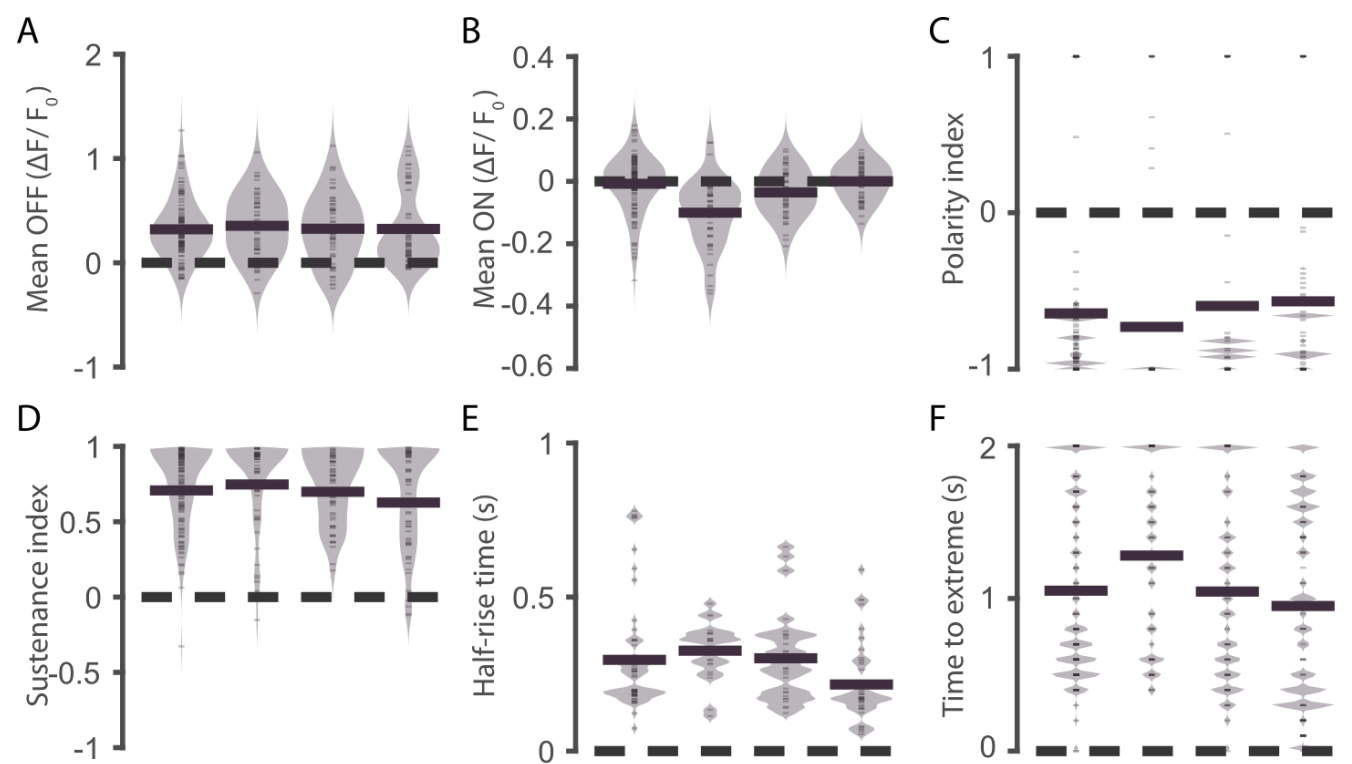

$\mathrm{E}$
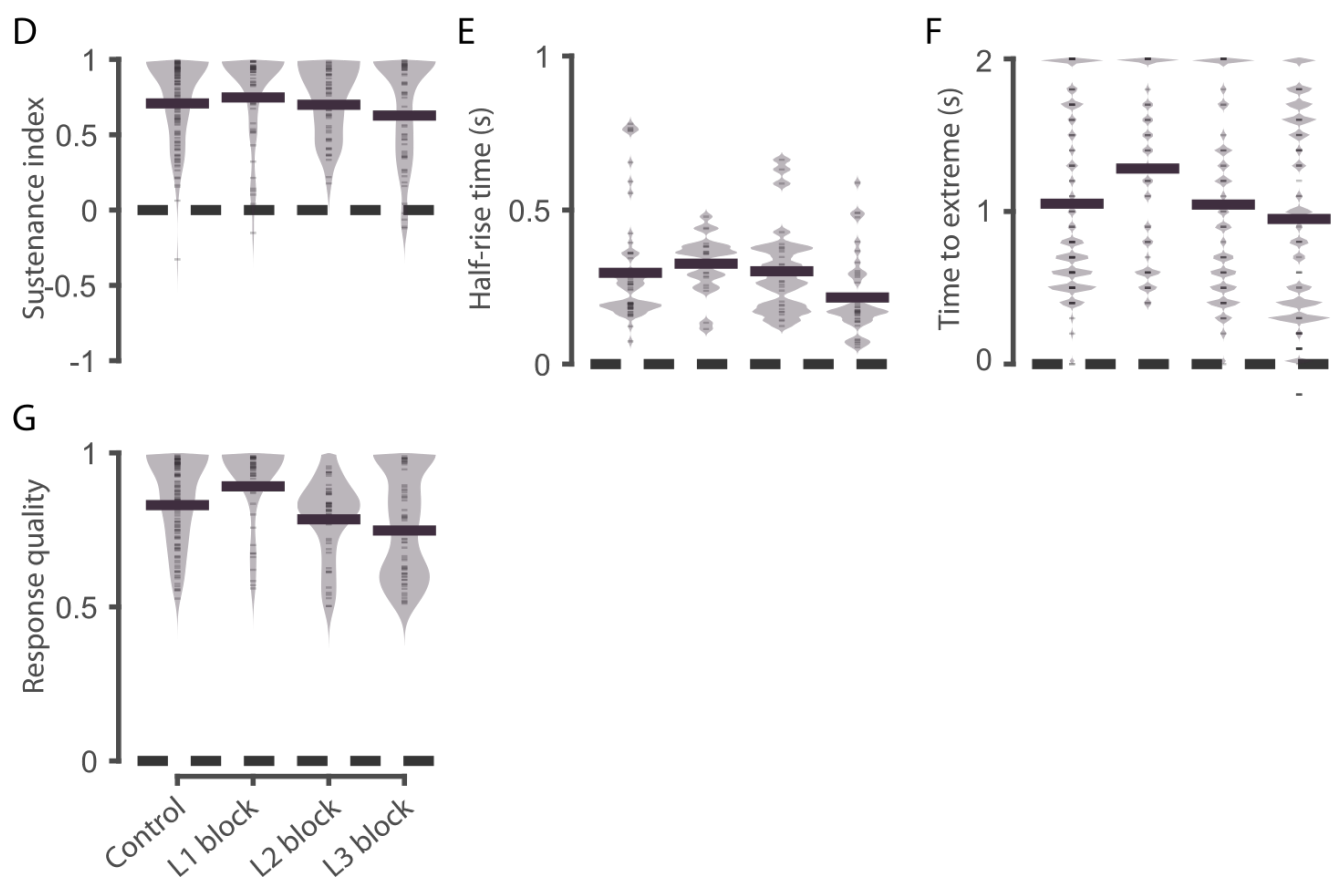

Figure 3.9: $\operatorname{Tm} 9$ response properties to ON-OFF full-field flashes without input from lamina neurons L1, L2, or L3.

Data is from fig. 3.8, but only neurons with response quality above 0.5 are used. Order in all plots is control vs L1, L2, or L3 block. A. Mean response to OFF. B. Mean response to ON. C. Polarity index. D. Sustenance index. E. Half-rise time. F. Time to extreme. G. Response quality. 


\section{Contribution of lamina cell types}

We start by the main inputs to the motion pathways, lamina neurons L1, L2, and L3, for which we have evidence of functional connectivity to Tm9. L3 is the strongest synaptic partner to Tm9. Both L1 and L3 shaped Tm9 response amplitude to ON-OFF flashes without intermediate gray in a different experimental setting (Fisher, Leong, et al., 2015). And L2 optogenetic activation led to positive Tm9 responses.

\section{Role of lamina neurons in Tm9 full-field responses}

We recorded responses to ON-OFF full-field flashes (fig. 3.8). Blocking L1 inputs decreased Tm9 responses to ON flashes but did not change the polarity index (fig. 3.9 B, C). Also, Tm9 neurons with L1 block peaked later without changing their sustenance (fig. 3.9 D, F). The response quality increased for L1 block compared to control (fig. $3.9 \mathrm{G}$ ). Blocking L2 inputs slightly decreased Tm9 responses to ON flashes (by $0.03 \frac{\Delta F}{F_{0}}$ ) without changing the polarity index, and lowered the response quality (fig. 3.9 B, C, G). Finally, blocking L3 inputs decreased the half-rise time and the response quality (fig. 3.1 D, G, tab. 3.1). Tm9 neurons without L3 inputs responded additionally to the OFF to gray, and ON to gray transitions, as seen in the average response trace (fig. $3.8 \mathrm{D}, \mathrm{H}$ ), this was not further investigated. The mean OFF response was unaltered upon L3 block, because a subset of neurons with high responses balanced a subset of neurons with low responses, resulting in a bimodal distribution (fig. 3.8 $\mathrm{D}, 3.9 \mathrm{~A}$ ). The lower part of the bimodal distribution of Tm9 responses under L3 block would be consistent with a strong reduction expected by connectomics, optogenetics, and above mentioned experiments using different stimulus, silencing strategy, and data selection (Fisher, Leong, et al., 2015). Here we showed: L1 inputs contribute to Tm9 rectification of ON responses, both L1 and L3 affect Tm9 OFF response kinetics, and L3 affects the distribution of OFF response amplitudes.

\section{Role of lamina neurons in $\mathrm{Tm} 9$ receptive fields}

Surprisingly, no strong effects were observed for full-field responses of Tm9 lacking lamina inputs. We next analyzed the responses of the same neurons to ON and OFF bars. As before (see p. 34), we visualized all Tm9 receptive fields for all experimental conditions. We aligned the OFF receptive fields by maximizing cross-correlation to neurons close to the center of the screen. The shift in position obtained from the OFF receptive fields was used to align the ON receptive fields. Most control neurons and 
L3 block ones had defined OFF receptive fields (fig. 3.10 A, D). On the contrary, fewer neurons of L2 block condition had defined OFF receptive fields (fig. $3.10 \mathrm{C}$ ).

Response amplitude to bars To check whether the lamina inputs shaped Tm9 response amplitude to bars, a localized stimulus, we plotted the extreme responses of Tm9 tuning curves. This is a non-parametric analysis independent of the Gaussian fit used to quantify the size of the receptive fields. This way we can observe neurons that were responding reliably (response quality larger than 0.5), even when the tuning curve could not be fit well by a single Gaussian. This is of particular interest, because some neurons had a ON receptive field that was negative in the center and positive in the surround. In some of those cases, the surround had larger magnitude than the center response resulting in positive extremes in fig. 3.11 C-D.

Visual inspection showed Tm9 responses to OFF bars were enhanced by blocking L1 inputs (fig. 3.11 A-B), and diminished by blocking L2 inputs (fig. 3.11 A-B). Again, effects were smaller for L3 block. However, examining Tm9 responses to ON bars revealed a role of L3 inputs. The control distribution of response extremes was bimodal, a group of neurons dominated by positive ON surround responses, and another group dominated by negative $\mathrm{ON}$ center responses. On average the extreme was close to zero. The mean extreme value was more negative for neurons with L2 or L3 block, because fewer neurons had positive ON surround responses (fig. 3.11 C-D). Taken together, L1 neurons reduced Tm9 responses to OFF bars, while L2 neurons increased them. Furthermore, L2 and L3 shaped the surround of Tm9 ON receptive fields.

Receptive field width We analyzed the receptive field of a reduced number of neurons that were fitted well by a single Gaussian. Less than half and less than a third of the recorded cells were used for the OFF and ON bars, respectively. Blocking lamina neurons did not impact much the proportion with respect to control for OFF bars. For ON bars, blocking input from L1, L2, or L3 reduced proportion of fitted cells to about a quarter, an eight, and a thirteenth, respectively (fig. 3.12 A-D). Unless the neuronal block is reversible and recordings are performed on the same neurons for control and block conditions, non-responsive cells exist in any fly depending on cells' health and the relative position of the stimulus and their receptive fields. Here as in (Fisher, Leong, et al., 2015), the proportion of Tm9 responsive neurons was lowest under L3 block. 
Table 3.2: Statistics for Tm9 receptive field widths under block of lamina neurons L1, L2, or L3.

Shown are p-values from two-sample two-sided permutation test of mean differences between control and the condition mentioned. Mean difference is FWHM $\left(^{\circ}\right)$ of control minus L1-, L2-, or L3-input block.

\begin{tabular}{|c|c|c|c|c|c|c|}
\hline & \multicolumn{2}{|c|}{ L1 block } & \multicolumn{2}{|c|}{ L2 block } & \multicolumn{2}{|c|}{ L3 block } \\
\hline & p-value & Mean diff & p-value & Mean diff & p-value & Mean diff \\
\hline$\overline{\text { OFFX }}$ & $3.50 \cdot 10^{-3}$ & $3-2.55$ & $3.12 \cdot 10^{-1}$ & $-8.53 \cdot 10^{-1}$ & $6.42 \cdot 10^{-1}$ & $-3.09 \cdot 10^{-1}$ \\
\hline OFFY & 0.00 & -3.55 & $2 \cdot 10^{-4}$ & -2.34 & $4.80 \cdot 10^{-3}$ & -1.43 \\
\hline ONX & $5.97 \cdot 10^{-1}$ & $1-1.08$ & $8.31 \cdot 10^{-1}$ & $-4.97 \cdot 10^{-1}$ & $4 \cdot 10^{-4}$ & -7.93 \\
\hline ONY & $3.10 \cdot 10^{-3}$ & $3-4.25$ & $2.27 \cdot 10^{-2}$ & -4.76 & 0.00 & -7.06 \\
\hline
\end{tabular}

Out of the responsive cells, the OFF receptive field width increased for both orientations under L1-input block (fig. 3.12 A, B). For vertical OFF bars receptive field width of Tm9 increased for all conditions: L1-, L2-, or L3-input blocks (fig. 3.12 A, B). Already the receptive field width is larger for horizontal receptive field than vertical bars in the control condition, we did not pursue this asymmetry further. For both orientations, blocking L1-input increased OFF receptive field size (fig. 3.12 A, B), and blocking L3-input increased ON receptive field size (fig. 3.12 C, D).

To test the effects on variability of receptive field properties, we looked at the coefficient of variation of the full width at half maximum (fig. 3.12 E-H). Overall, we observed no statistically significant effect. The coefficient of variation was lowered by L2-input block for ON bars of both orientations resulting from a decreased standard deviation and increased mean (only for vertical bars, fig. $3.12 \mathrm{G}, \mathrm{H}, \mathrm{K}, \mathrm{L}$ ). However, the small sample size precludes rigorous analysis of variability.

In conclusion, silencing lamina neurons led to reduced amount of responsive neurons, particularly for ON stimuli. From the responsive neurons, Tm9 neurons lacking L1 inputs had larger OFF receptive fields, and Tm9 neurons lacking L3 inputs had larger ON receptive fields. We detected no significant effect on receptive field size variability. Lack of L1 inputs also increased the amplitude of responses to OFF bars, thus L1 could provide indirect inhibition to Tm9 OFF receptive fields in the center and surround. The lack of this OFF surround inhibition from L1 could account for wider Tm9 receptive fields. Similarly, blocking L3 decreased the surround responses of Tm9 ON receptive fields, accounting for wider Tm9 ON receptive fields. This suggested two diverging pathways from L1 and L3 converge back at Tm9, one mediates excitatory center responses, the other mediates inhibitory surround responses. 
A

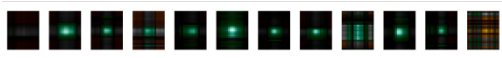

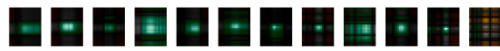

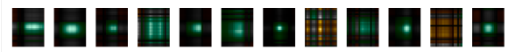

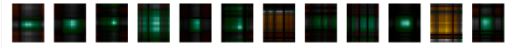

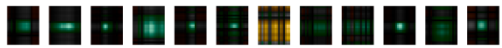

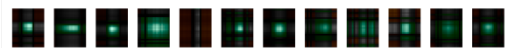

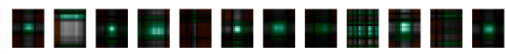

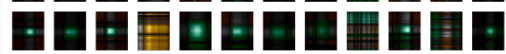

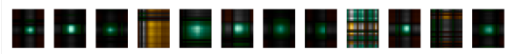

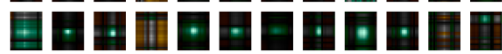

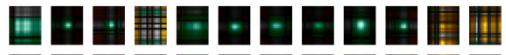

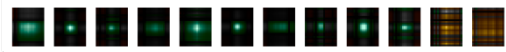

B

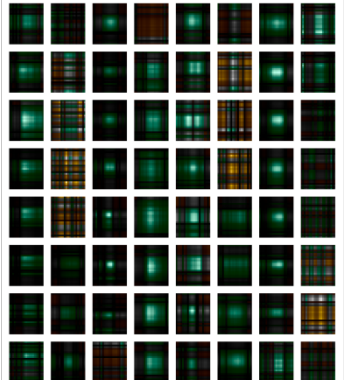

C

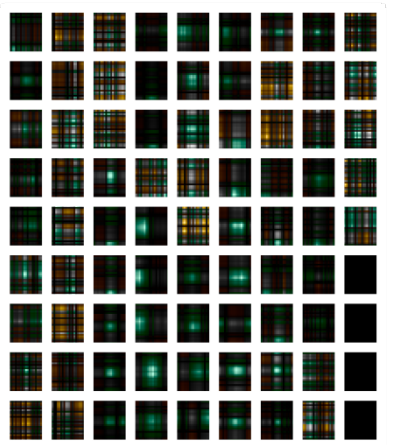

D

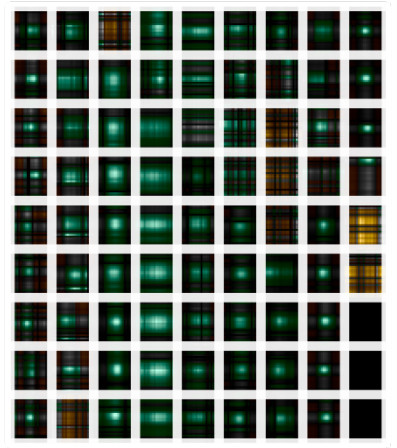

E

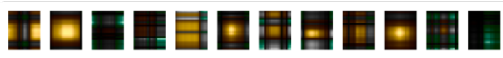

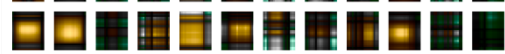

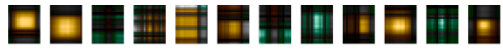

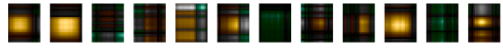

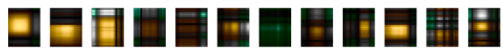

Control

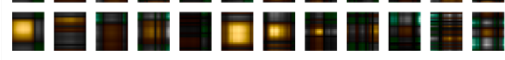

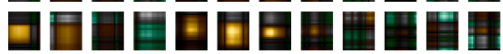

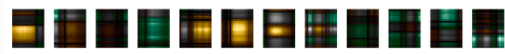

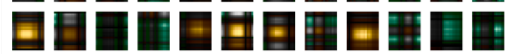

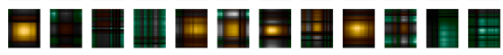

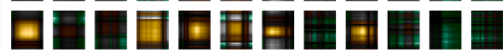

$\mathrm{F}$

L1 block

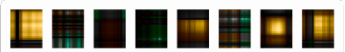

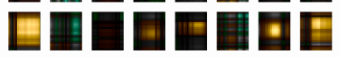

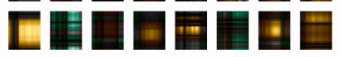

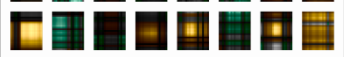
둡ㅁㅁㅂㅛ 붐물

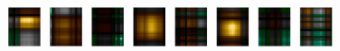
目田雨回的

G

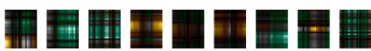

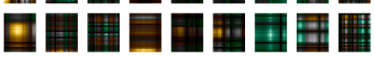

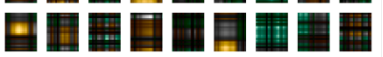

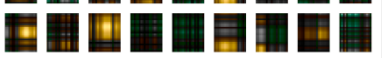

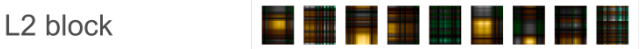

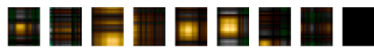

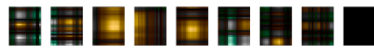

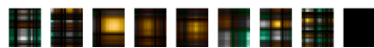

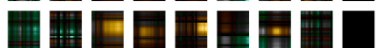

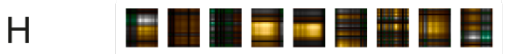

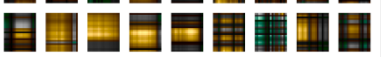

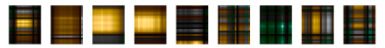

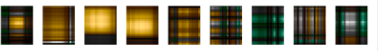

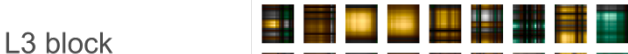

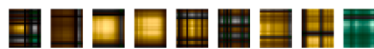
围国回国国国

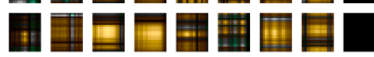

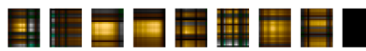

Figure 3.10: Receptive field width of Tm9 upon silencing single lamina cell types (L1, L2, or L3).

A-D. Receptive fields of Tm9 neurons to OFF bars for control (A), L1-input block (B), L2-input block (C), and L3-input block (D). E-H. Receptive fields of Tm9 neurons to ON bars matching A-D. 
Tuning curve peak responses to ON and OFF bars

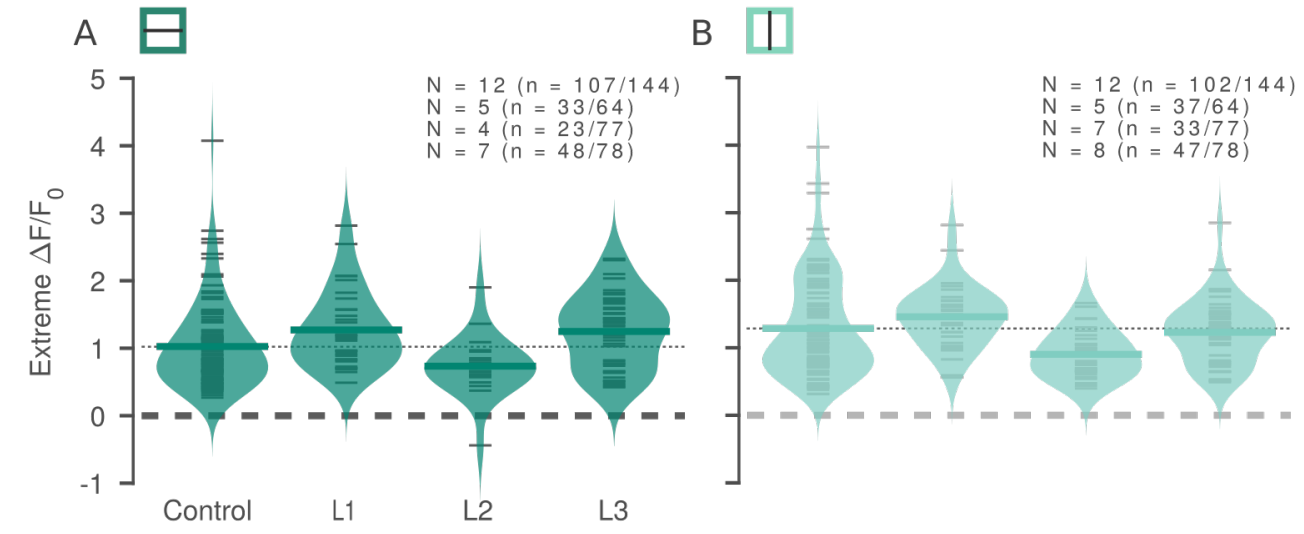

B I

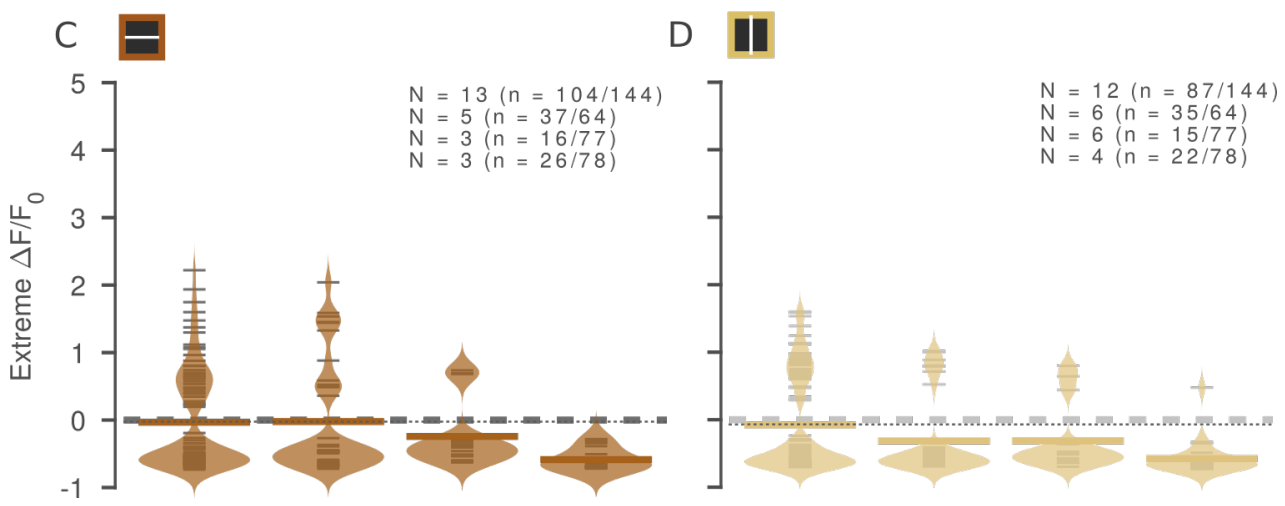

Figure 3.11: Receptive field amplitude distribution of Tm9 upon silencing single lamina cell types L1, L2 or L3.

A-D. Distribution of receptive field extreme values of responses to: horizontal OFF bars (A), vertical OFF bars (B), horizontal ON bars (C), vertical ON bars (D). All stimuli were recorded for the same neurons, but only the neurons with response quality over 0.5 were used for each panel. $\mathrm{N}$ is number of flies from which the $\mathrm{n}$ plotted neurons come / number of recorded neurons. 


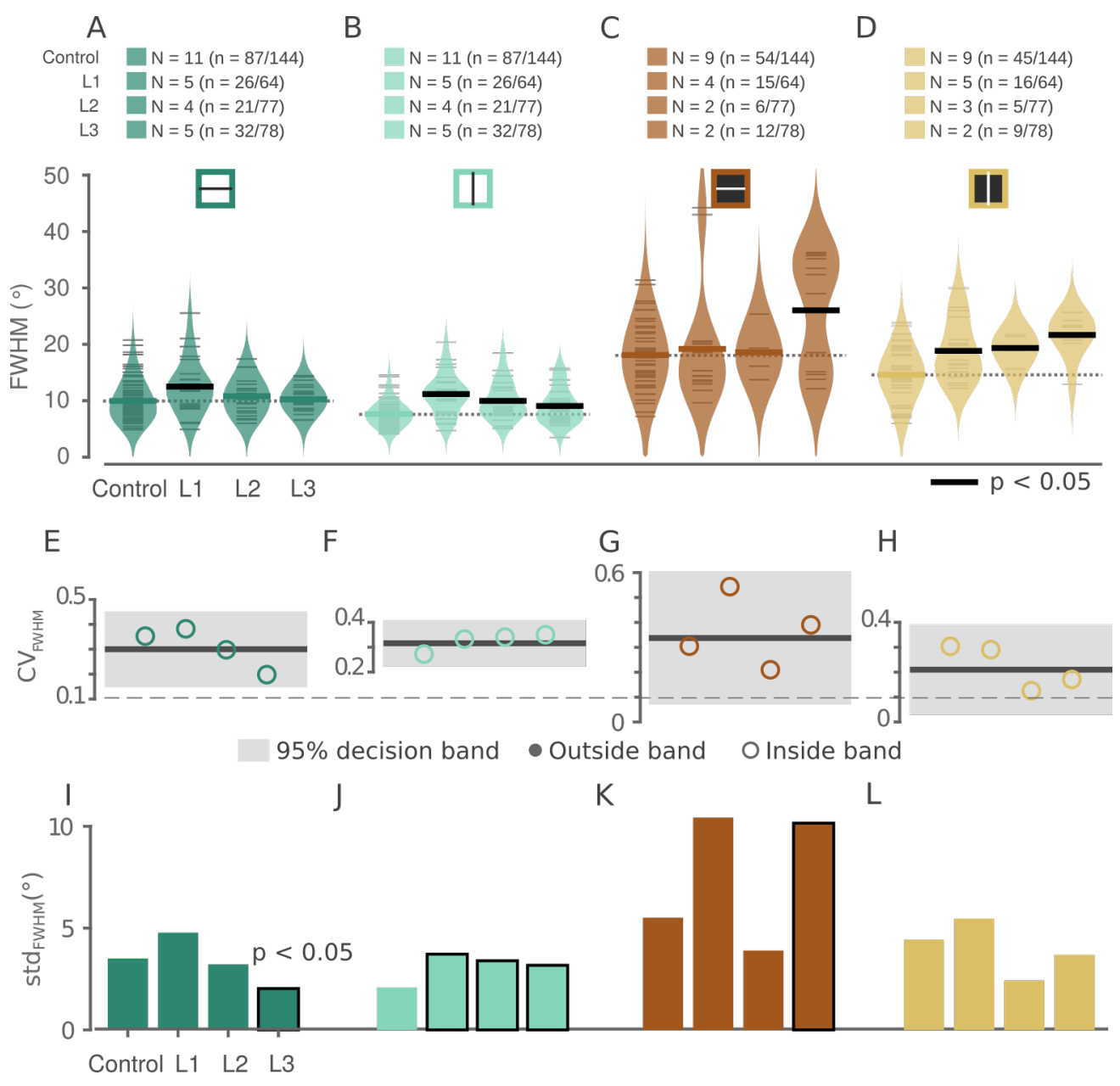

Figure 3.12: Receptive field width distribution of Tm9 upon silencing single lamina cell types (L1, L2 or L3).

A-D. Distribution of receptive field widths from FWHM of responses to: horizontal OFF bars (A), vertical OFF bars (B), horizontal ON bars (C), vertical ON bars (D). E-H. Coefficient of variation from distributions in A-D. Line is the average coefficient of variation for all conditions, and the band shows the 95\% bootstrapped confidence interval, open and closed circles indicate values inside and outside the band, respectively. I-L. Standard deviation of distributions in A-D. 


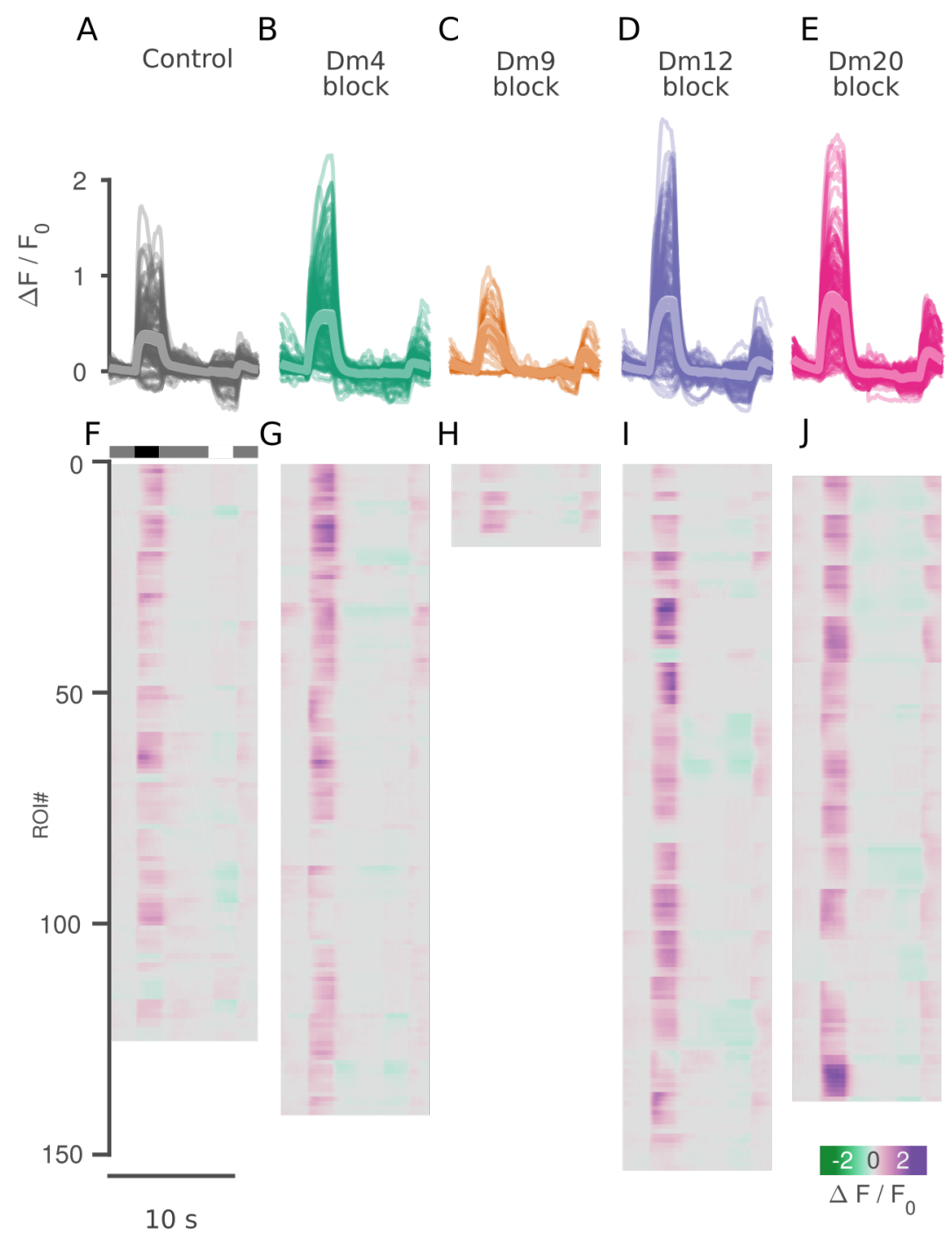

Figure 3.13: Tm9 responses to ON-OFF full-field flashes without input from wide field neurons Dm4, Dm9, Dm12, or Dm20.

A-E. Trial averaged responses to a sequence of gray-OFF-gray-ON, of $4 \mathrm{~s}$, $2 \mathrm{~s}, 4 \mathrm{~s}$ and $2 \mathrm{~s}$ duration, respectively. Thin lines indicate single neurons, on top is the mean and standard error. Controls (A), Dm4 block (B), Dm9 block (C), Dm12 block (D), and Dm20 block (E). F-J. Traces in A-E stacked for better visualization of different response kinetics. 

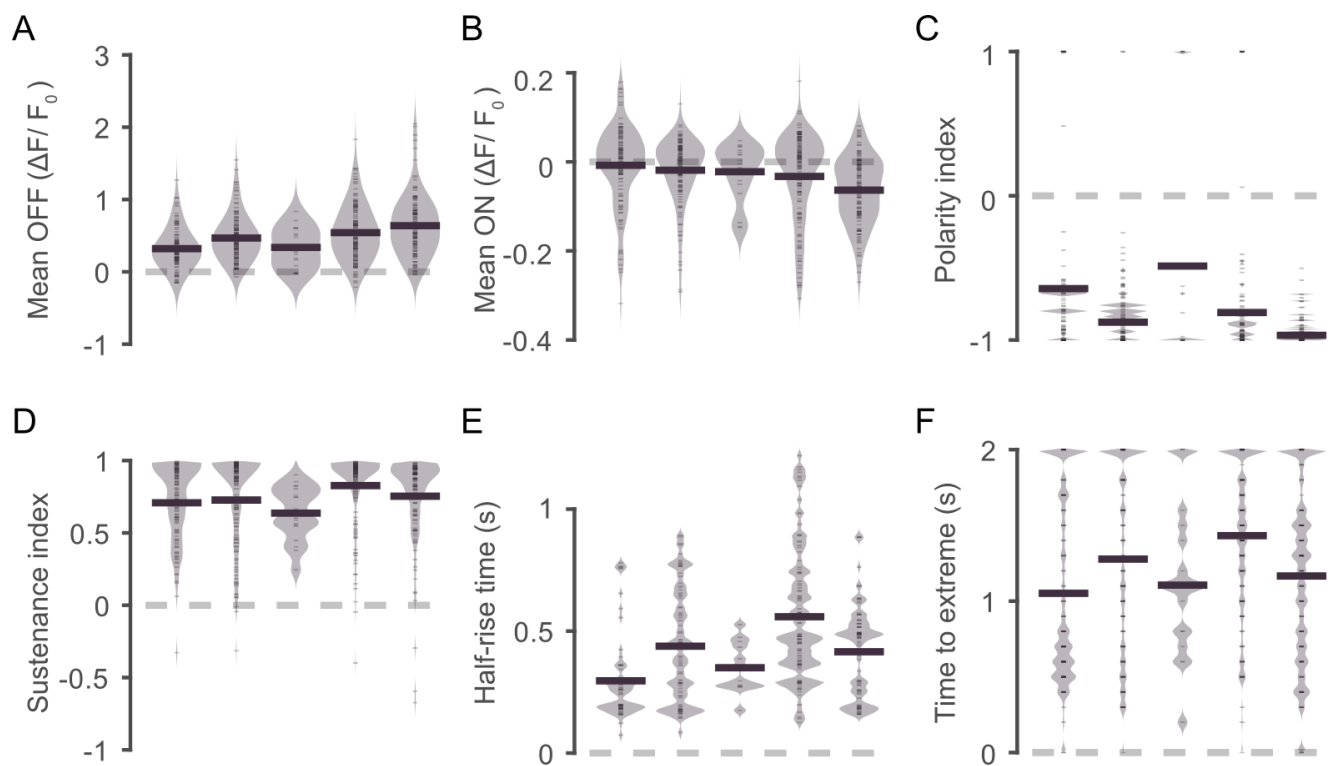

$\mathrm{E}$
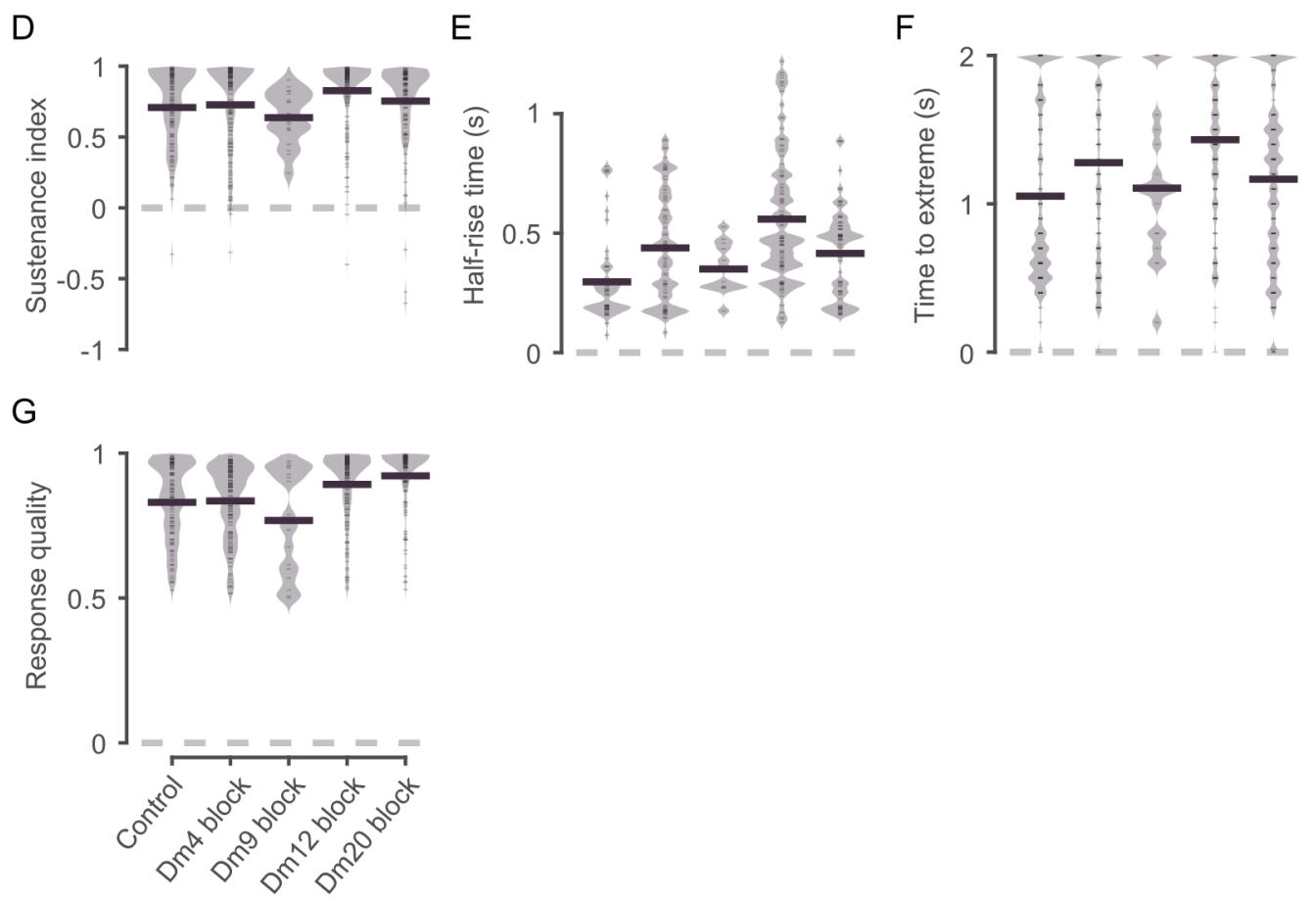

Figure 3.14: Tm9 response properties to ON-OFF full-field flashes without input from wide field neurons Dm4, Dm9, Dm12, or Dm20.

Data is from fig. 3.13, but only neurons with response quality above 0.5 are used. Order in all plots is control vs Dm4, Dm9, Dm12, or Dm20 block. A. Mean response to OFF. B. Mean response to ON. C. Polarity index. D. Sustenance index. E. Half-rise time. F. Time to extreme. G. Response quality. 


\section{Contribution of wide-field cell types}

Next, we studied how blocking the inputs from wide field neurons affected the receptive fields of Tm9 neurons. Besides the wide-field inputs from Dm4, Dm12, and Dm20 confirmed by optogenetics, we added preliminary data on Dm9, another distal medulla neuron that projects to layer M3. Dm9 could have a role in color vision in the fly, and potentially mediate Tm9 response properties through interactions with L3 and R8 photoreceptors.

Table 3.3: Statistics for Tm9 ON-OFF full-field flashes response properties under block of distal medulla neurons Dm4, Dm9, Dm12, or Dm20.

Shown are p-values from two-sample two-sided permutation test of mean differences between control and the condition mentioned. Mean difference is control minus Dm4-, Dm9-, Dm12- or Dm20-input block.

\begin{tabular}{|c|c|c|c|c|c|c|c|c|}
\hline & \multicolumn{2}{|c|}{ Dm4 block } & \multicolumn{2}{|c|}{ Dm9 block } & \multicolumn{2}{|c|}{ Dm12 block } & \multicolumn{2}{|c|}{ Dm20 block } \\
\hline & $\mathrm{p}$-value & Mean diff & $\mathrm{p}$-value & Mean diff & $\mathrm{p}$-value & Mean diff & $\mathrm{p}$-value & Mean diff \\
\hline Mean OFF $\left(\frac{\Delta F}{F}\right)$ & $1 \cdot 10^{-4}$ & $-1.50 \cdot 10^{-1}$ & $8.10 \cdot 10^{-1}$ & $-1.70 \cdot 10^{-2}$ & $2 \cdot 10^{-5}$ & $-2.20 \cdot 10^{-1}$ & $2 \cdot 10^{-5}$ & $-3.20 \cdot 10^{-1}$ \\
\hline Mean ON $\left(\frac{\Delta F^{p}}{F_{0}}\right)$ & $2.80 \cdot 10^{-1}$ & $1.10 \cdot 10^{-2}$ & $5.20 \cdot 10^{-1}$ & $1.40 \cdot 10^{-2}$ & $3 \cdot 10^{-2}$ & $2.50 \cdot 10^{-2}$ & $2 \cdot 10^{-5}$ & $5.60 \cdot 10^{-2}$ \\
\hline Polarity index & $1.40 \cdot 10^{-4}$ & $2.30 \cdot 10^{-1}$ & $3.80 \cdot 10^{-1}$ & $-1.60 \cdot 10^{-1}$ & $1.60 \cdot 10^{-2}$ & $1.70 \cdot 10^{-1}$ & $2 \cdot 10^{-5}$ & $3.20 \cdot 10^{-1}$ \\
\hline Sustenance index & $5.70 \cdot 10^{-1}$ & $-1.90 \cdot 10^{-2}$ & $2.50 \cdot 10^{-1}$ & $7.10 \cdot 10^{-2}$ & $8 \cdot 10^{-5}$ & $-1.20 \cdot 10^{-1}$ & $1.70 \cdot 10^{-1}$ & $-4.60 \cdot 10^{-2}$ \\
\hline Half-rise time (s) & $8 \cdot 10^{-5}$ & $-1.40 \cdot 10^{-1}$ & $3.70 \cdot 10^{-1}$ & $-5.40 \cdot 10^{-2}$ & $2 \cdot 10^{-5}$ & $-2.60 \cdot 10^{-1}$ & $8 \cdot 10^{-5}$ & $-1.20 \cdot 10^{-1}$ \\
\hline Time to extreme (s) & $4 \cdot 10^{-3}$ & $-2.30 \cdot 10^{-1}$ & $7.10 \cdot 10^{-1}$ & $-5.40 \cdot 10^{-2}$ & $2 \cdot 10^{-5}$ & $-3.80 \cdot 10^{-1}$ & $1.10 \cdot 10^{-1}$ & $-1.10 \cdot 10^{-1}$ \\
\hline Response quality & $7.60 \cdot 10^{-1}$ & $-4.70 \cdot 10^{-3}$ & $7 \cdot 10^{-2}$ & $6.30 \cdot 10^{-2}$ & $2 \cdot 10^{-5}$ & $-6.20 \cdot 10^{-2}$ & $2 \cdot 10^{-5}$ & $-9.10 \cdot 10^{-2}$ \\
\hline
\end{tabular}

\section{Role of distal medulla neurons in Tm9 full-field responses}

First we checked the effects on the responses to ON-OFF full-field flashes. Looking at all recorded neurons (fig. 3.13), we observed an increased response to OFF flashes for Dm4, Dm12, and Dm20 blocks. Also, the ON to gray response increased for all blocked neuron types. Analyzing the response properties in more detail ${ }^{2}$ revealed Tm9 responses to OFF flashes were indeed reduced when the input from Dm4, Dm12, or Dm20 was blocked (fig. 3.14 A, tab. 3.3). Also, blocking Dm12 or Dm20 decreased the responses to ON flashes (fig. $3.14 \mathrm{~B}$ ). The shifts in $\mathrm{ON}$ and OFF responses were accompanied by a polarity index with higher OFF preference (fig. $3.14 \mathrm{C}$ ). Blocking Dm12 made Tm9 responses to OFF flashes more sustained, with longer half-rise time and time to extreme (fig. 3.14 D-F). On the other hand, blocking Dm4 only increased half-rise time and time to extreme (fig. $3.14 \mathrm{E}, \mathrm{F}$ ), but not the sustenance index. And Dm20 block only increased the half-rise time (fig. 3.14 E). Tm9 neurons had larger response quality when Dm12 or Dm20 inputs were blocked (fig.

\footnotetext{
${ }^{2}$ We included only responsive neurons with response quality index grater than 0.5 .
} 
3.14 G). Thus, Dm12 modulates all studied properties of Tm9 responses to full-field flashes. Taken together, wide field neurons Dm4, Dm12, and Dm20 modulate the response polarity, and kinetics of Tm9.

\section{Role of distal medulla neurons in Tm9 receptive fields}

Table 3.4: Statistics for Tm9 receptive field widths under block of wide-field distal medulla neurons Dm4, Dm9, Dm12, or Dm20.

Shown are p-values from two-sample two-sided permutation test of mean differences between control and the condition mentioned. Mean difference is FWHM $\left(^{\circ}\right)$ of control minus Dm4-, Dm9-, Dm12- or Dm20-input block.

\begin{tabular}{|c|c|c|c|c|c|c|c|c|}
\hline & \multicolumn{2}{|c|}{ Dm4 block } & \multicolumn{2}{|c|}{ Dm9 block } & \multicolumn{2}{|c|}{ Dm12 block } & \multicolumn{2}{|c|}{ Dm20 block } \\
\hline & p-value & Mean diff & $\mathrm{p}$-value & Mean diff & p-value & Mean diff & p-value & Mean diff \\
\hline$\overline{\text { OFFX }}$ & $6.54 \cdot 10^{-2}$ & $8.46 \cdot 10^{-1}$ & $5.73 \cdot 10^{-1}$ & $-7.85 \cdot 10^{-1}$ & $1.17 \cdot 10^{-1}$ & $-8.36 \cdot 10^{-1}$ & $6.45 \cdot 10^{-1}$ & $-2.40 \cdot 10^{-1}$ \\
\hline OFFY & $1.75 \cdot 10^{-1}$ & $-4.28 \cdot 10^{-1}$ & 0.00 & -6.75 & $6.00 \cdot 10^{-3}$ & -1.01 & $1.57 \cdot 10^{-1}$ & $-4.35 \cdot 10^{-1}$ \\
\hline ONX & $4.49 \cdot 10^{-1}$ & $-6.95 \cdot 10^{-1}$ & - & - & 0.00 & -4.68 & $1 \cdot 10^{-4}$ & -5.54 \\
\hline ONY & $2.50 \cdot 10^{-3}$ & -2.84 & - & - & 0.00 & -4.02 & 0.00 & -5.71 \\
\hline
\end{tabular}

We proceeded to look into the receptive field properties of Tm9 upon blocking one of the wide field input neuron types. We visualized the population of recorded cells in fig. 3.15. Note that for Dm9 the sample size was about a quarter from the one of control condition. Rfs of flies with Dm12 or Dm20 inputs blocked seemed larger, more so for ON than for OFF bars. The proportion of responding cells to ON bars seemed higher than control when Dm12 input was blocked.

For further analysis we fitted a Gaussian to $\mathrm{Tm} 9$ receptive fields. There were no strong effects in the response amplitude of the Gaussian fits. Only Dm20 block raised responses to OFF bars of both orientations, but only significantly for vertical bars (fig. 3.16 A-B, E-F). Blocking Dm12 slightly decreased Tm9 responses to vertical OFF bars (fig. 3.16 B, E-F).

The receptive fields showed no increase in size for OFF bars, except few neurons with Dm9-input block for vertical bars (fig. 3.17 B). Due to lower sample size, no further conclusion will be drawn from Dm9-input block experiments. Blocking Dm4 inputs increased receptive field size only for vertical ON bars (fig. 3.17 D). Moreover, blocking either Dm12 or Dm20 inputs to Tm9 increased the receptive field size for ON stimuli in both orientations (fig. 3.17 C, D).

About variability of the receptive field size, no change in the coefficient of variation was significant (fig. $3.17 \mathrm{E}-\mathrm{H}$ ). Nevertheless, the coefficient of variation was lower for ON stimuli under Dm4-, or Dm12-input block (fig. $3.17 \mathrm{G}, \mathrm{H}$ ). This is explained by a combination of increased mean and decreased standard deviation in the receptive field size distribution 
(fig. 3.17 C, D, K, L). Blocking Dm20 had opposing effects, the increased size of the ON receptive field was accompanied by an increased standard deviation, thus having no effect on the coefficient of variation (fig. 3.17 C, D, G, H, K, L).

Taken together, our data suggested Dm12 and Dm20 inputs constrain the ON receptive field size of Tm9. And Dm4 and Dm12 reduce the variability of size of the $\mathrm{ON}$ receptive fields of $\mathrm{Tm} 9$ relative to the mean, while $\mathrm{Dm} 20$ increases variability via the variance of the size distribution. 
A

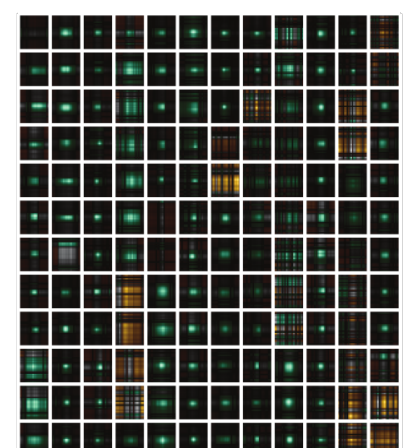

B

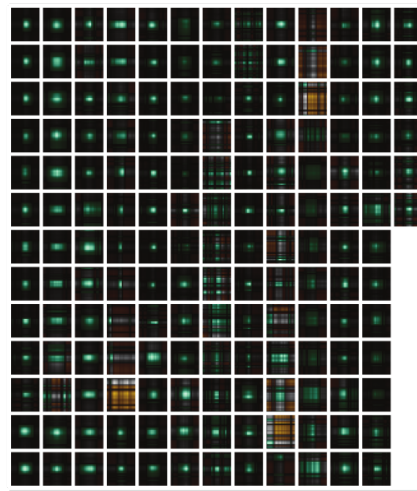

C

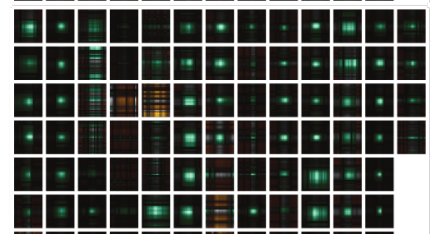

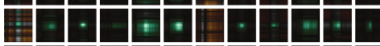

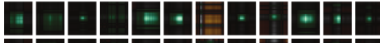

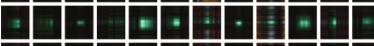

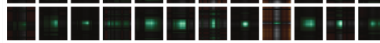

$+.-2 .+1.5$

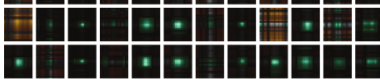

$\mathrm{D}$

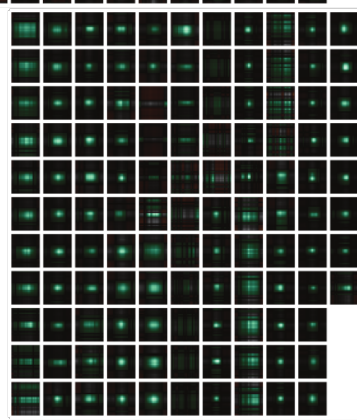

E

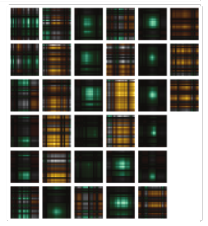

Receptive fields

OFF ON

- 트

$50\left(^{\circ}\right)$

Control

Dm4 block

Dm12 block

Dm20 block

Dm9 block
F

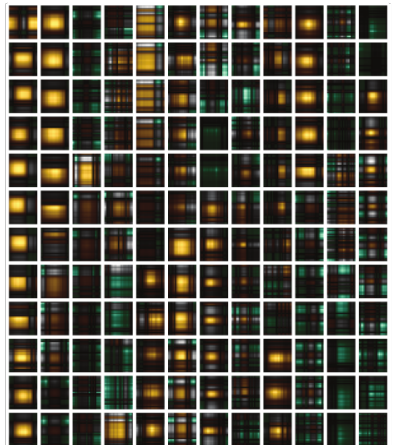

G

-

in-

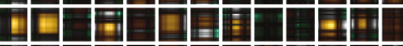

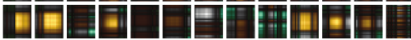

或国

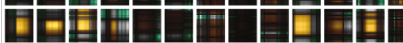

H-

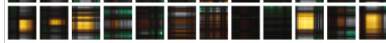

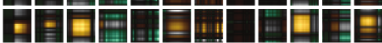

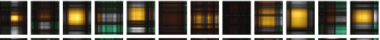

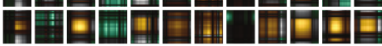

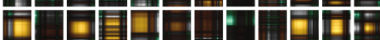

的星目

$\mathrm{H}$

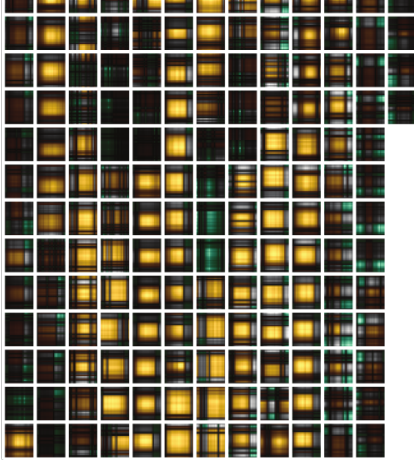

I

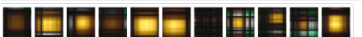

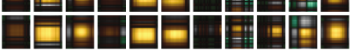

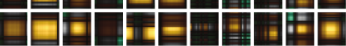

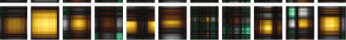

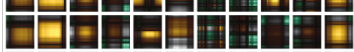

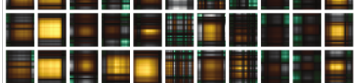

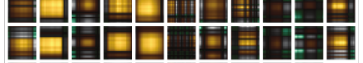

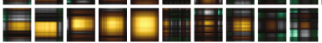

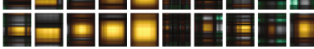

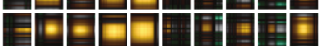

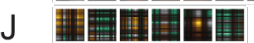

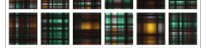

- +4 i

国国到

T+1

Figure 3.15: Receptive field of Tm9 upon silencing single distal medulla cell types.

Controls (A, F), Dm4 block (B, G), Dm12 block (C, H), Dm20 block (D, I) or Dm9 block (E, J). Left and right columns are for OFF and ON stimuli, respectively. 
Tm9 receptive field amplitude without wide-field inputs

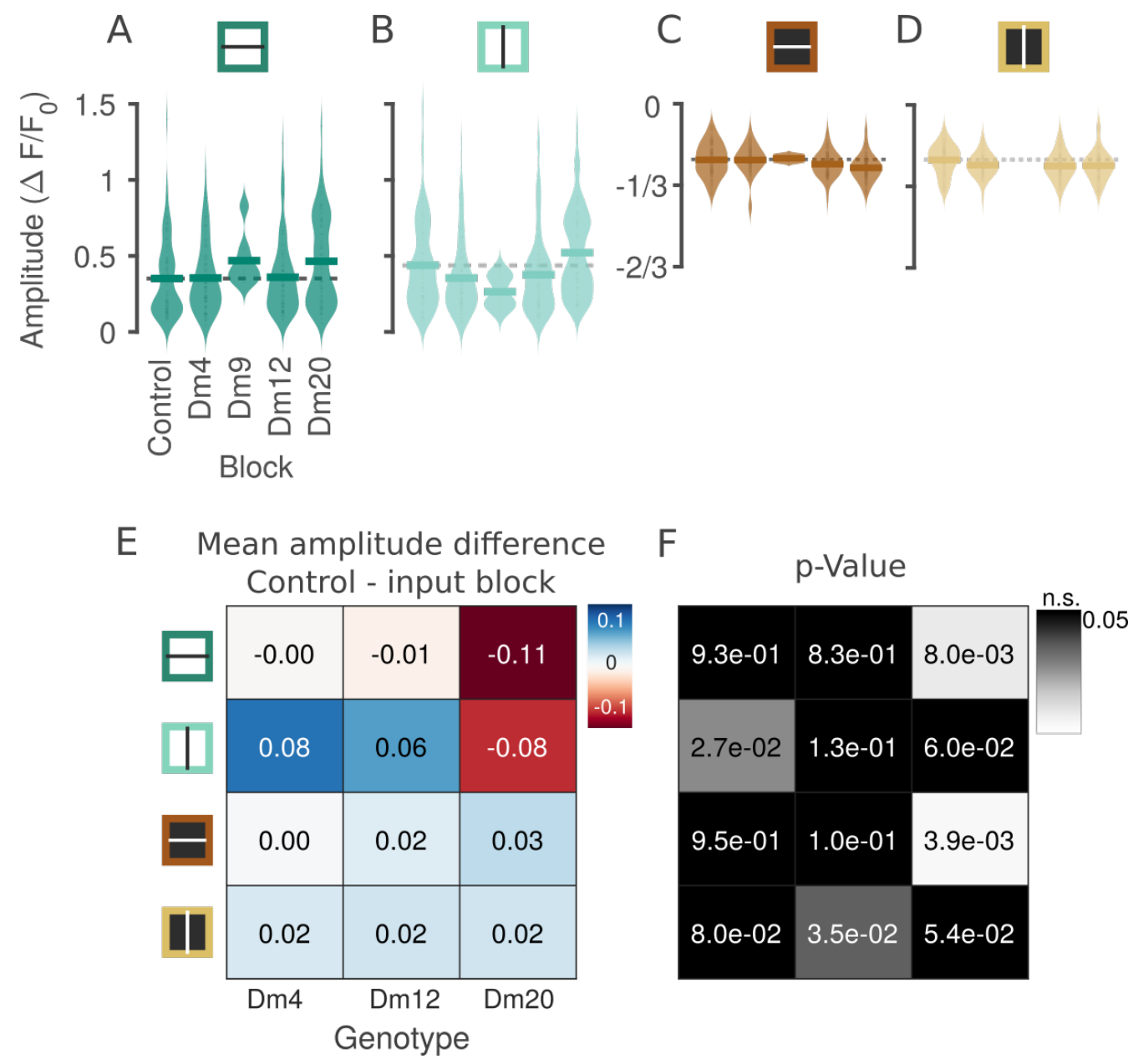

Figure 3.16: Receptive field properties of responsive Tm9 neurons upon blocking Dm4, Dm9, Dm12, or Dm20 neurons.

A-D. Receptive field amplitude of Tm9 for control vs Dm4, Dm9, Dm12, or Dm20 input block for: OFF horizontal (A) and vertical bars (B); ON horizontal (C) and vertical bars (D). E. Mean amplitude difference between control and the experimental conditions. Decrements and increments with respect to control are in blue and red, respectively. F. P-values from permutation tests of mean differences in (E). Values above 0.05 are in black and highlight non-significant (n.s.) values. 


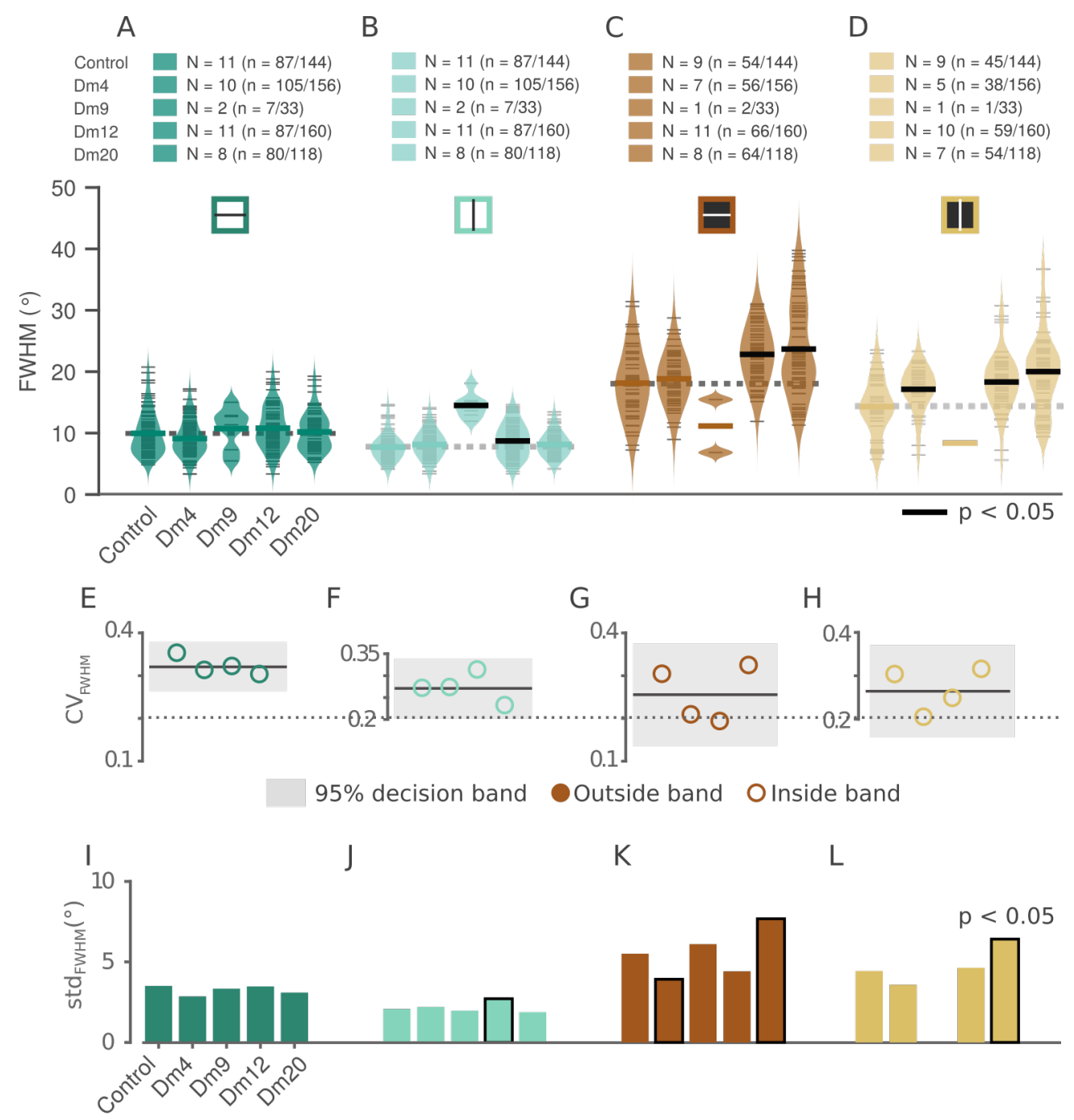

Figure 3.17: Receptive field width of Tm9 upon silencing single distal medulla cell types (Dm4, Dm9, Dm12, or Dm20).

A-D. Distribution of receptive field widths from FWHM of responses to: horizontal OFF bars (A), vertical OFF bars (B), horizontal ON bars (C), vertical ON bars (D). E-H. Coefficient of variation from distributions in (A-D). Line is the average coefficient of variation for all conditions, and the band shows the 95\% bootstrapped confidence interval, open and closed circles indicate values inside and outside the band, respectively. I-L. Standard deviation of distributions in (A-D). 


\subsection{Role of electrical coupling in the receptive field of Tm9 neurons}

We have seen Tm9 neurons were very robust to single cell type manipulations. This suggested multiple cell types contribute to the ON and OFF receptive field properties of Tm9. To test broader contributions, we could block the transmission of a specific input channel, for example pharmacologically blocking glutamate receptors. Another broad manipulation would be to block gap junctions responsible for electrical coupling between neurons. Gap junctions are formed by two pores, one in each membrane of the connected cells. The pores for gap junctions in invertebrates are made out of proteins of the innexin family (Phelan, Stebbings, et al., 1998; Phelan and Starich, 2001).

Therefore, we studied the effect of gap junction block on the response properties of Tm9. One of the main innexins in Drosophila melanogaster is shaking-B (shakB) (Phelan, Stebbings, et al., 1998). We used RNAi silencing of $\operatorname{shakB}$ in Tm9 neurons, thereby eliminating gap junctions requiring shakB in Tm9 membrane. This will be referred to as gap junction block in the rest of the section. We must note that other gap junction forming proteins (such as ogre) may remain in Tm9.

Table 3.5: Statistics for Tm9 ON-OFF full-field flashes response properties under gap junction block.

Shown are p-values from two-sample two-sided permutation test of mean differences between control and the condition mentioned. Mean difference is control minus Tm9-shakB-RNAi.

\begin{tabular}{lcr}
\hline & p-value & Mean difference \\
\hline Mean OFF $\left(\frac{\Delta F}{F_{0}}\right)$ & $9.51 \cdot 10^{-1}$ & $3.50 \cdot 10^{-3}$ \\
Mean ON $\left(\frac{\Delta F}{F_{0}}\right)$ & $7.70 \cdot 10^{-3}$ & $4.78 \cdot 10^{-2}$ \\
Polarity index & $4.30 \cdot 10^{-1}$ & $7.05 \cdot 10^{-2}$ \\
Sustenance index & $6.73 \cdot 10^{-1}$ & $-1.75 \cdot 10^{-2}$ \\
Half-rise time $(\mathrm{s})$ & $2.39 \cdot 10^{-1}$ & $1.11 \cdot 10^{-1}$ \\
Time to extreme $(\mathrm{s})$ & $2.52 \cdot 10^{-1}$ & $-9.01 \cdot 10^{-2}$ \\
Response quality & $5.26 \cdot 10^{-1}$ & $8.90 \cdot 10^{-3}$ \\
\hline
\end{tabular}

First, we observed the effects of gap junction block on ON-OFF full-field flashes responses. The gap junction block decreased responses to ON full-field flashes, but did not affect OFF responses (fig. 3.18, 3.19). 

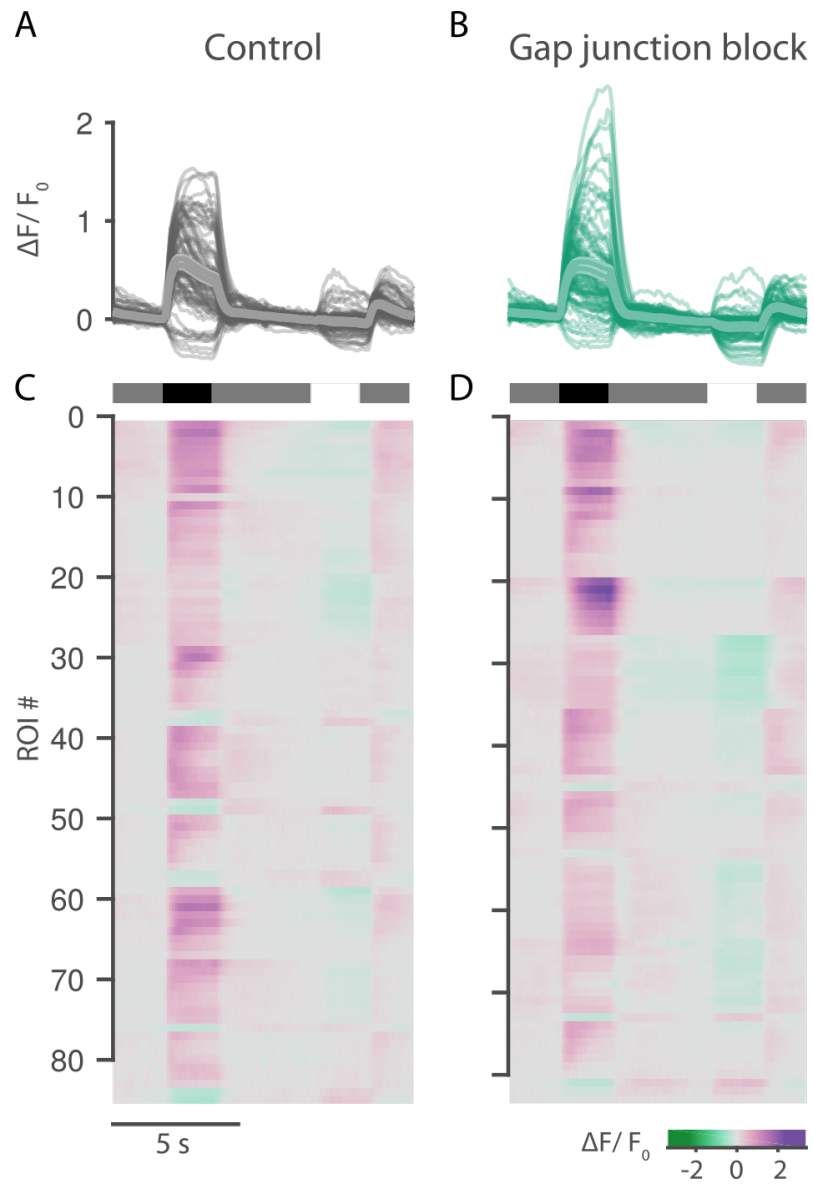

Figure 3.18: Tm9 responses to ON-OFF full-field flashes upon RNAi silencing of shakB in Tm9 neurons.

A-B. Trial averaged responses to a sequence of gray-OFF-gray-ON, of $4 \mathrm{~s}$, $2 \mathrm{~s}, 4 \mathrm{~s}$ and $2 \mathrm{~s}$ duration, respectively. Thin lines indicate single neurons, on top is the mean and standard error. (A) Controls. (B) Tm9-shakB-RNAi. C-D. Traces in A and B stacked for better visualization of different response kinetics.

Next, we examined the contribution of shakB-mediated gap junctions to $\mathrm{Tm} 9$ receptive field properties. The response amplitude to vertical OFF bars decreased upon gap-junction block (fig. $3.21 \mathrm{~B}$ ). On the other hand, the response amplitude to $\mathrm{ON}$ bars did not change (fig. $3.21 \mathrm{C}, \mathrm{D}$, tab. 3.6). Gap junction block increased the receptive field size for vertical ON bars and also slightly for OFF bars, but not for horizontal bars (fig. 3.21 E-H). Blocking gap junctions also increased the variance of the receptive field size distribution, thereby increasing the coefficient of variation for all 
A

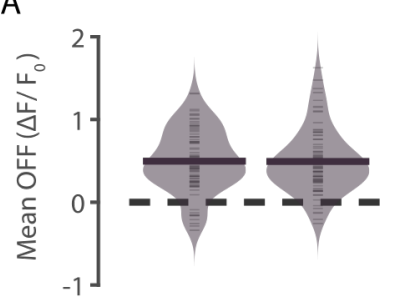

D

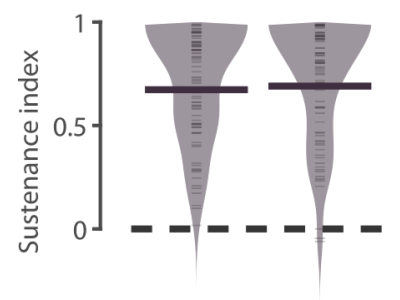

G

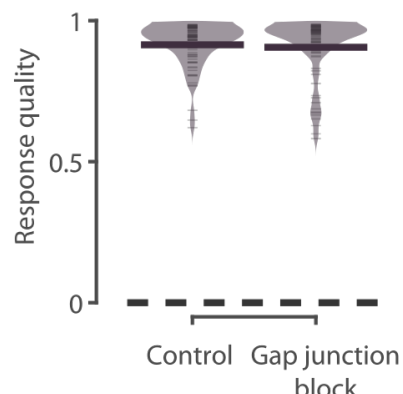

B

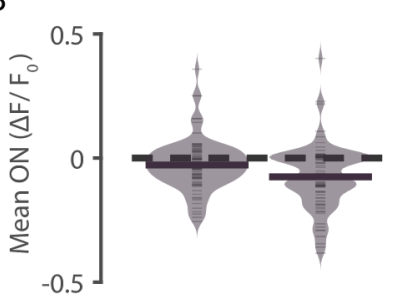

E

E
C

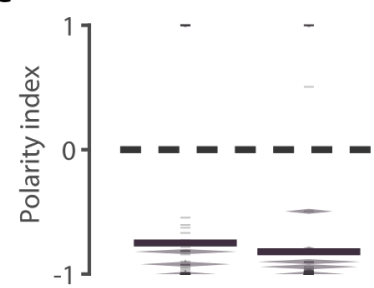

$\mathrm{F}$

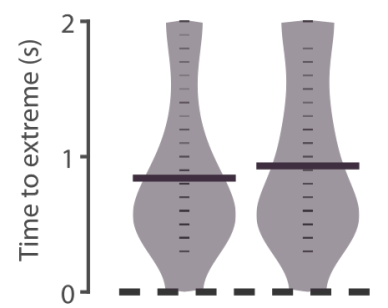

Figure 3.19: Tm9 response properties to ON-OFF full-field flashes upon RNAi silencing of shakB in Tm9 neurons from fig. 3.18.

Only neurons with response quality above 0.5 are used. Order in all plots is control vs Tm9-shakB-RNAi. A. Mean response to OFF. B. Mean response to ON. C. Polarity index. D. Sustenance index. E. Half-rise time. F. Time to extreme. G. Response quality.

stimuli (fig. 3.21 I-P, tab. 3.6). Therefore, electrical synapses sharpen the variance of the $\mathrm{Tm} 9$ receptive field size distribution, and the symmetry of the receptive field by narrowing vertical ON receptive fields. 


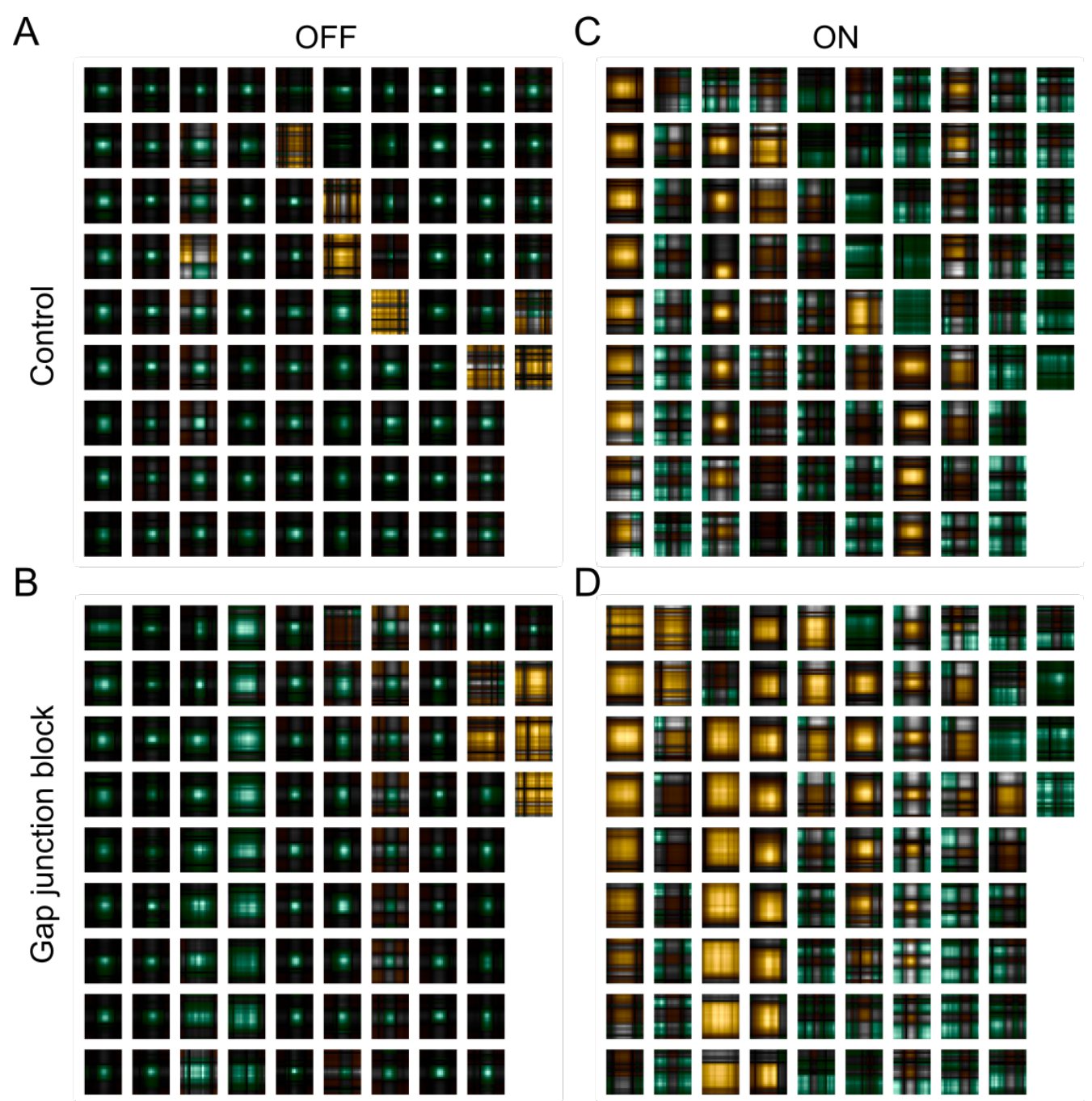

Figure 3.20: Receptive fields of Tm9 upon RNAi silencing of shakB in Tm9 neurons.

A-B. Receptive fields of Tm9 neurons to OFF bars for control (A) and shakB-RNAi (B). C-D. Similar to A-B but for ON bars. 
A

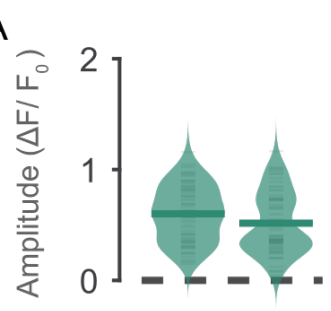

E

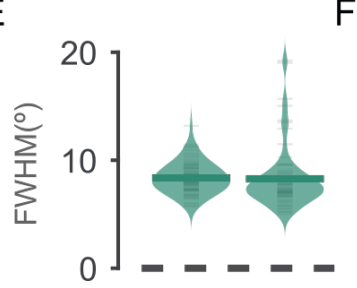

|

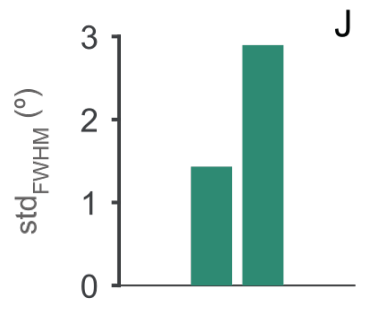

M

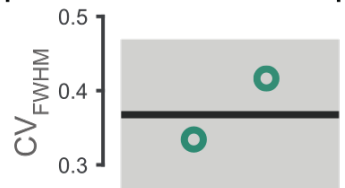

$\mathrm{N}$

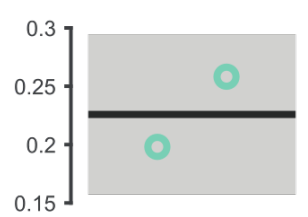

B

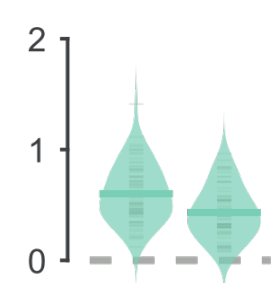

$\mathrm{F}$
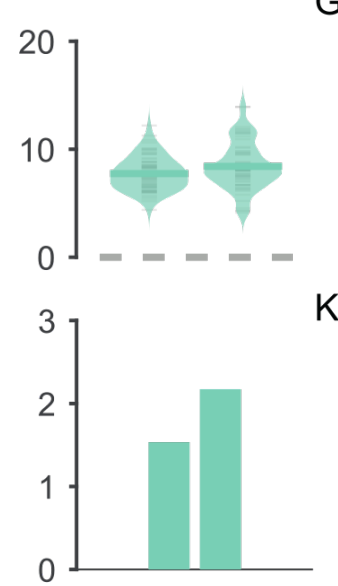

o

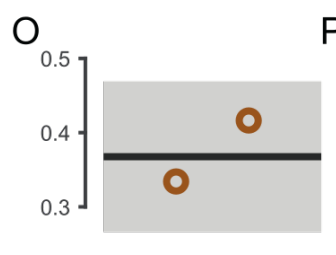

D

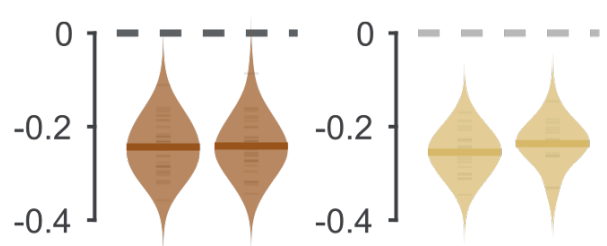

$\mathrm{H}$

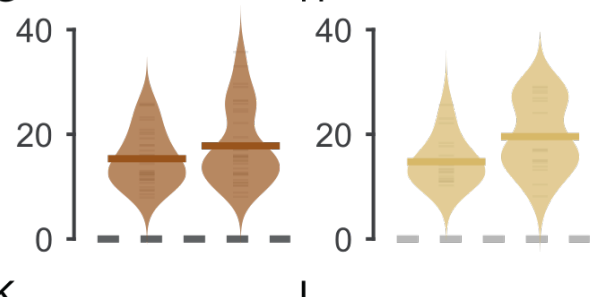

L

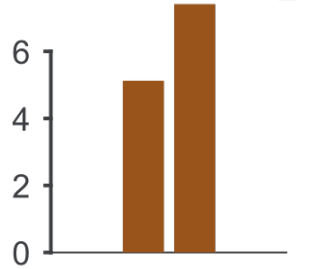

$\mathrm{P}$
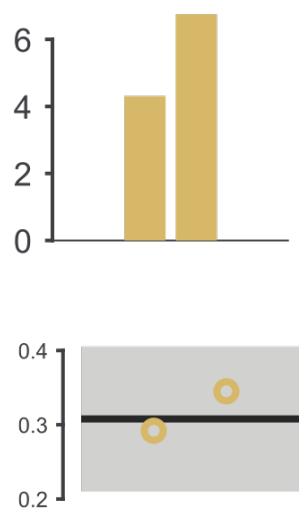

Figure 3.21: Receptive field properties of responsive Tm9 neurons upon RNAi silencing of shakB in Tm9 neurons.

A-D. Receptive field amplitude of control vs Tm9 gap junction block (Tm9shakBRNAi) for: OFF horizontal (A) and vertical bars (B); ON horizontal (C) and vertical bars (D). E-H. Similar as A-D but for the receptive field size. I-L. Standard deviation of the FWHM distributions in (E-H). M-P. Coefficient of variation of the FWHM distributions in (E-H), gray band is the $95 \%$ decision band, no change was significant. 
Table 3.6: Statistics for $\mathrm{Tm} 9$ receptive field size under gap junction block.

Shown are p-values from two-sample two-sided permutation test of mean differences between control and the condition mentioned. Mean difference is control minus Tm9-shakB-RNAi. Variance of the full width at half maximum, variance equality was tested with the Brown-Forsythe test, shown are the p-value and the ratio of variances: control divided by Tm9-shakB-RNAi.

\begin{tabular}{|c|c|c|c|c|}
\hline & \multicolumn{2}{|c|}{ OFF } & \multicolumn{2}{|c|}{$\mathrm{ON}$} \\
\hline & Horizontal & Vertical & Horizontal & Vertical \\
\hline $\begin{array}{l}\text { Amplitude }\left(\frac{\Delta F}{F_{0}}\right) \\
\text { p-value } \\
\text { Mean difference }\end{array}$ & $\begin{array}{l}6 \cdot 10^{-2} \\
8 \cdot 10^{-2}\end{array}$ & $\begin{array}{l}1 \cdot 10^{-4} \\
2 \cdot 10^{-1}\end{array}$ & $\begin{array}{r}9 \cdot 10^{-1} \\
-2 \cdot 10^{-3}\end{array}$ & $\begin{array}{r}2 \cdot 10^{-1} \\
-2 \cdot 10^{-2}\end{array}$ \\
\hline $\begin{array}{l}\text { FWHM }\left(^{\circ}\right) \\
\text { p-value } \\
\text { Mean difference }\end{array}$ & $\begin{array}{l}8 \cdot 10^{-1} \\
8 \cdot 10^{-2}\end{array}$ & $\begin{array}{l}3 \cdot 10^{-2} \\
-6.70 \cdot 10^{-1}\end{array}$ & $\begin{aligned} & 1 \cdot 10^{-1} \\
- & 2.46\end{aligned}$ & $\begin{aligned} & 1 \cdot 10^{-2} \\
- & 4.80\end{aligned}$ \\
\hline $\begin{array}{l}\text { Var(FWHM) } \\
\text { p-value } \\
\text { Var ratio }\end{array}$ & $\begin{array}{l}3.96 \cdot 10^{-2} \\
2.44 \cdot 10^{-1}\end{array}$ & $\begin{array}{l}5.26 \cdot 10^{-2} \\
4.99 \cdot 10^{-1}\end{array}$ & $\begin{array}{l}1.49 \cdot 10^{-1} \\
4.78 \cdot 10^{-1}\end{array}$ & $\begin{array}{l}3.98 \cdot 10^{-2} \\
4.08 \cdot 10^{-1}\end{array}$ \\
\hline
\end{tabular}




\section{$-4-$ \\ Contributions of Tm9 and related wide-field neurons to motion detection}

In the previous chapter, we demonstrated that wide-field neurons play an important role modulating the receptive field properties of $\mathrm{Tm} 9$ neurons. First, we identified novel wide-field inputs to Tm9. Then, optogenetic experiments showed wide-field neurons have inhibitory effects in Tm9. Finally, we recorded $\mathrm{Tm} 9$ responses while blocking wide-field inputs and showed that they modulated the size of the $\operatorname{Tm} 9$ ON receptive fields. Because Tm9 is a crucial neuron in the OFF motion pathway, it is likely that the effects of wide-field inputs on Tm9 can propagate downstream in the circuit. Therefore, in this chapter we studied the role of wide-field neurons in the response properties of direction-selective neurons, namely T4 and T5.

\subsection{Receptive fields of distal medulla neurons}

We started by doing a basic characterization of Dm4 and Dm12 neurons ${ }^{1}$. We recorded Dm4 and Dm12 neurons expressing GCaMP6f using in vivo two-photon calcium imaging. First, we visualized their responses to ONOFF full-field flashes. Both neurons responded with larger amplitude to OFF flashes (fig. 4.1 A, B). Dm12 OFF responses raised slowly and reached maximum at the offset of the stimulus, ON responses raised faster and were sustained (fig. 4.1 A). Both ON and OFF Dm4 responses reached

\footnotetext{
${ }^{1}$ These experiments were done based on positive GRASP results of Dm4 and Dm12. In the meantime, I supervised a master student (Cagatay Aydin) while he recorded receptive fields of Dm4, Dm9, Dm12, and Dm20. The stimulus used there had a gray background, here we used full contrast bars consistently with all other receptive field recordings in this thesis. Receptive fields widths for OFF bars were about $7^{\circ}, 10^{\circ}$ and $15^{\circ}$ for Dm4, Dm12, and Dm20, respectively.
} 
maximum faster than Dm12 and sustained the activity throughout the stimulus (fig. $4.1 \mathrm{~B}$ ). Therefore, Dm4 and Dm12 responded preferentially to OFF stimuli with slow and sustained kinetics.

We next recorded receptive fields from responses to vertical ON and OFF bars. Both neurons responded to positively to OFF bars, and negatively to ON bars. Receptive fields of dendritic segments of Dm4 were arranged retinotopically, and were larger for $\mathrm{ON}$ than for OFF bars (fig. $4.2 \mathrm{~B}$, C). Despite the wide-field anatomy of Dm4, receptive fields of dendritic segments were restricted to one eye column, the FWHM from Gaussian fits was about $5^{\circ}$ for OFF bars (data not shown, fig. 4.2 A, B). On the other hand, receptive fields of Dm12 dendritic segments were wider than one column and overlapped with neighboring receptive fields (fig. 4.2 D-F). Full width at half maximum of Gaussian fits to Dm12 receptive fields were about $20^{\circ}$ and $30^{\circ}$ for OFF and ON bars, respectively (data not shown). Thus, wide-field neurons can process information in different spatial scales, Dm4 acts locally within one eye column, and Dm12 signaling spreads over multiple columns.

A

Dm12 ON-OFF responses

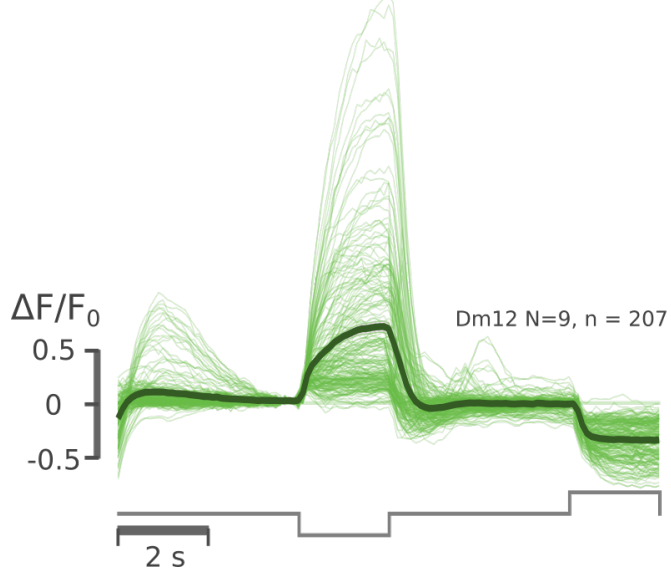

B

Dm4 ON-OFF responses

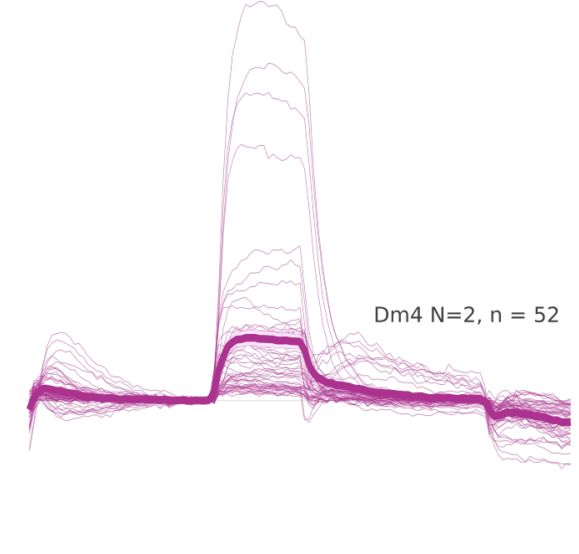

Figure 4.1: Dm4 and Dm12 responses to ON-OFF full-field flashes. Normalized fluorescence from neurons expressing GCaMP6f. Individual thin lines are single neuron responses, thick lines are the neuron average, standard error of the mean is similar to the average line thickness. A. Responses of Dm12. B. Responses of Dm4. 


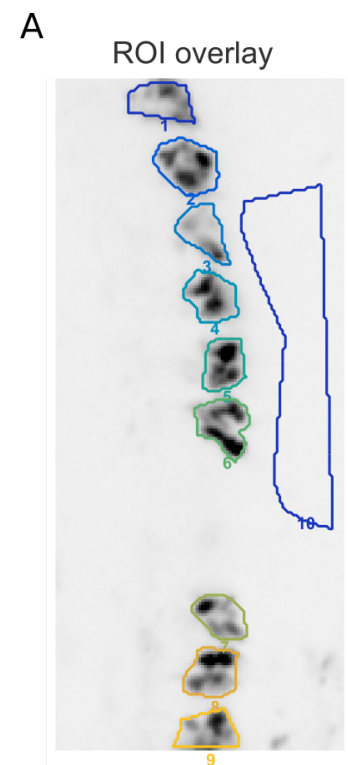

B Dm4 vertical OFF receptive fields

$\mathrm{D}$

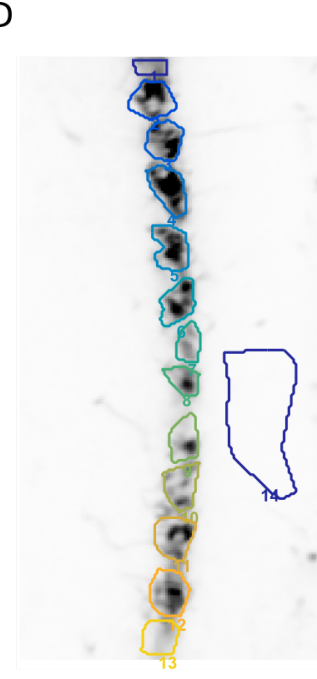

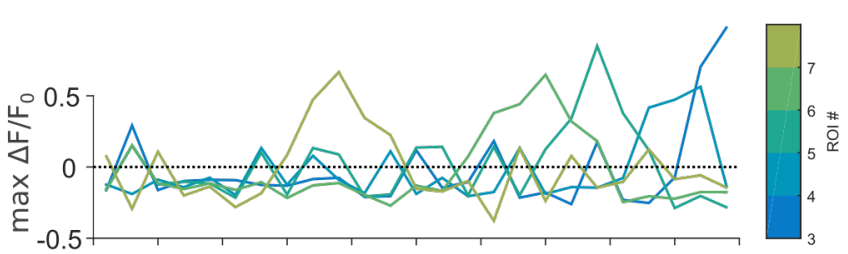

C Dm4 vertical ON receptive fields

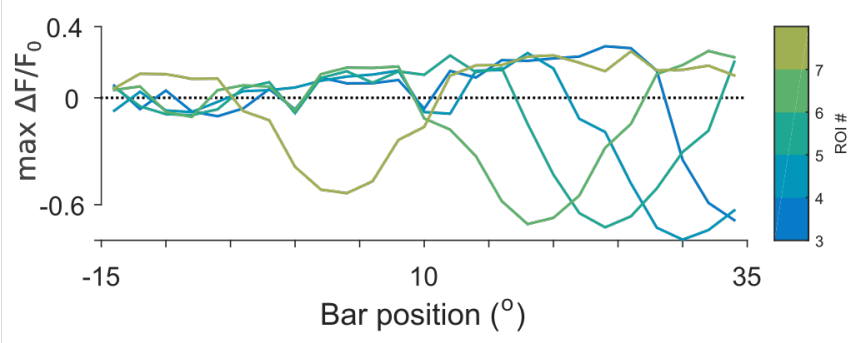

$\mathrm{E}$
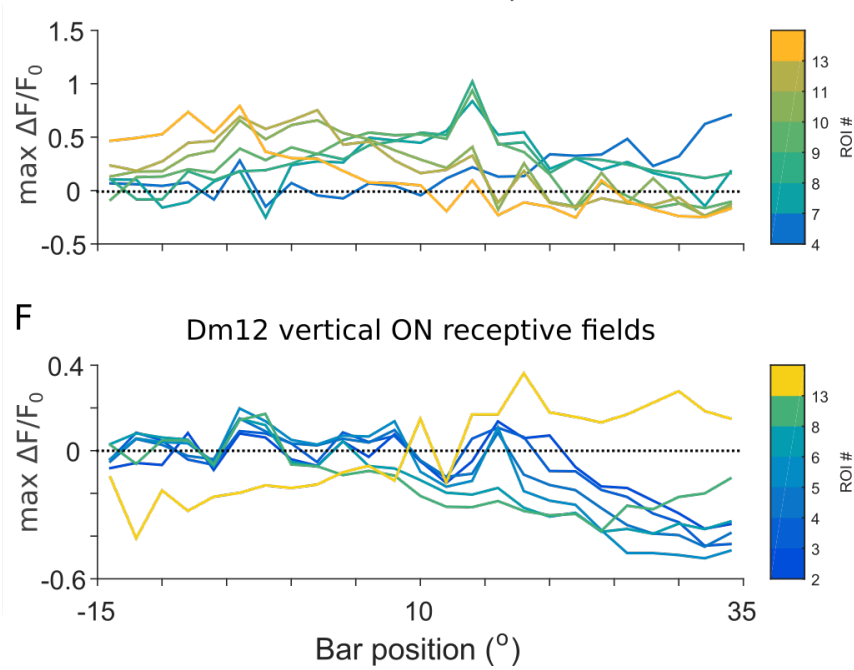

Figure 4.2: Dm4 receptive fields are restricted to one eye column and Dm12 receptive fields span multiple columns.

Normalized fluorescence from neurons expressing GCaMP6f. A, D.Maximum intensity projection of recording for (A) Dm4 and (B) Dm12. B, C. Dm4 receptive fields for vertical OFF bars (B) and ON bars (C) from selected regions-of-interest in (A). Notice the retinotopy that continues even after a gap in the GCaMP6f expression of Dm4. Each dendritic segment acts as a local processing unit, their receptive field is restricted to one eye column. Dm4 ON receptive fields are wider than OFF ones. E, F. Dm12 receptive fields for vertical OFF bars (E) and ON bars (F) from selected regions-of-interest in (D). Dm12 dendritic receptive fields span more than one column (FWHM $i 5^{\circ}$ ) and are wider for ON than for OFF. 


\subsection{Wide-field inputs tune responses of direction-selective neurons}

After sketching the response properties of some wide-field neurons, we tested how Tm9 and its wide-field inputs modulate the properties of direction-selective neurons. To this end, we silenced the activity of the distal medulla neurons Dm4, Dm12, Dm20, or Tm9, and recorded the activity in the direction-selective neurons $\mathrm{T} 4$ and $\mathrm{T} 5$. We recorded the activity of both T4 and T5 neurons, because no specific line was available to target only either of them. This offers the advantage of additionally studying effects on the ON motion-pathway. As before, neuron activity was blocked by overexpression of the inward-rectifying Kir2.1 channel. Responses of T4 and T5 neurons were recorded using in vivo two-photon calcium imaging, with GCaMP6f expression in T4 and T5. Recordings focused on the lobula plate, where T4 and T5 axon terminals lie (fig. 4.3 A).

We measured responses to ON and OFF edges moving in four different directions (up, down, left, right). To select regions of interest, every pixel was correlated with its nearest neighbors. The mean correlation with the nearest neighbors gave a correlation map with local structure, i.e., clumps of pixels behaving similarly. From this correlation map, we selected circular regions-of-interest of $2.75 \mu \mathrm{m}$ diameter to include at most one axon terminal (fig. $4.3 \mathrm{~B}$ ), which are less than $5 \mu \mathrm{m}$ in diameter (Maisak et al., 2013). Because the neurites of T4 and T5 are intertwined in the lobula plate, regions-of-interest were a mixture of pixels from either neuron. T4 neurons respond selectively to moving ON edges, and T5 neurons to moving OFF edges. Thus, we used the contrast preference of the region-of-interest to get an idea of the mixture of T4 an T5 per region-of-interest. 


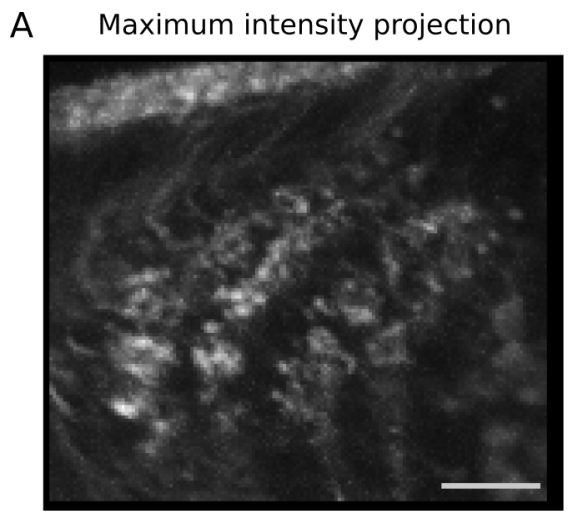

B Nearest-neighbor correlation map

C Direction preference map (DPM)
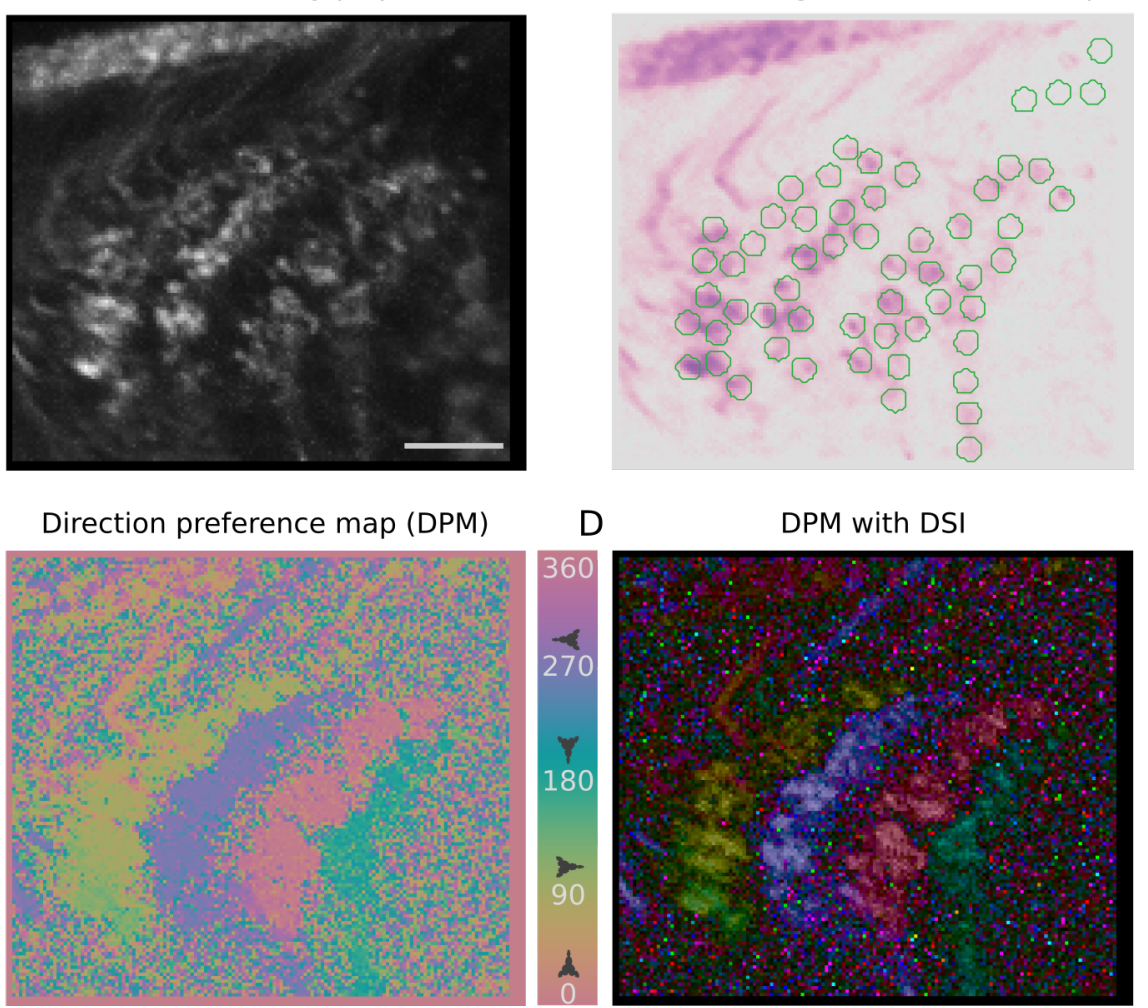

D

DPM with DSI
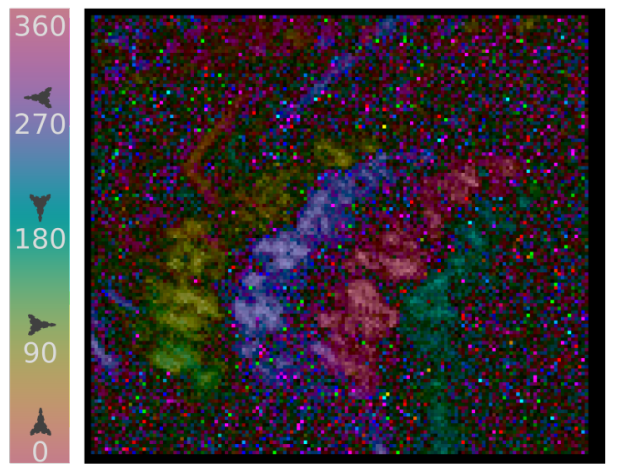

Figure 4.3: T4 and T5 response maps to moving edges.

A. Maximum intensity projection of the time series of an example fly. Scale bar is $10 \mu \mathrm{m}$. B. Nearest-neighbor correlation map, darker colors indicate higher correlations. Selected ROIs are overlaid in green. C. Direction preference map (DMP), each pixel was color coded in the HCL color space (Hughes and Goodhill, 2015), hue is preferred direction of the pixel, chroma and lightness are same for all pixels. D. Same map as (C) but encoding the direction selectivity index (DSI) in the color lightness, brighter colors indicate higher DSI. Notice the nice separation of preferred directions across the four layers of the lobula plate.

\section{Response amplitude and contrast selectivity}

To quantify preference to ON or OFF edges, we used a contrast selectivity index (eq. 6.11). This index is bound between $[-1,1],-1$ is pure OFF preference, 1 is pure ON preference, and 0 is no preference. The contrast selectivity index of control T4 and T5 responses was positive. Thus, there was a bias towards ON edges, i.e., T4 responses (fig. 4.4). This ON bias was further increased by blocking the activity of any of the neuron types 
Dm4, Dm12, Dm20, or Tm9 (fig. 4.4, tab. 4.1).

To find the cause of the contrast selectivity index increments, we studied the responses to ON and OFF edges separately. The response to each polarity was calculated by averaging the maximum response across all directions for a given polarity. Blocking any of the neurons affected T4 and T5 responses (fig. 4.5) . Blocking the activity of Dm12, Dm20, or Tm9 increased responses to moving ON edges, and decreased responses to moving OFF edges. Blocking Dm4 activity decreased responses to both ON and OFF moving edges (fig. 4.6, tab. 4.2).

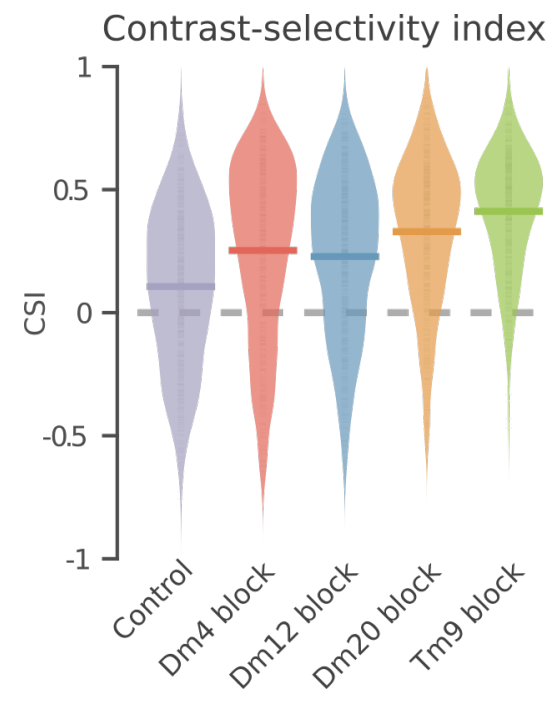

Figure 4.4: T4 and T5 contrast selectivity increased upon blocking activity of Dm4, Dm12, Dm20, or Tm9.

T4 and T5 contrast selectivity to moving edges for control, and block of Dm4, Dm12, Dm20, or Tm9. All conditions lead to an increase of the contrast selectivity, i.e., preference to ON edges (Table 4.1) . 
Table 4.1: Statistics for T4 and T5 contrast selectivity to moving edges under input block: Dm4, Dm12, Dm20, or Tm9.

Shown are difference of means: control minus condition, and corresponding p-values from two-sample two-sided permutation test of mean differences.

\begin{tabular}{lrr}
\hline & \multicolumn{1}{c}{ p-value } & Mean difference \\
\hline Dm4 block & $2.00 \cdot 10^{-5}$ & $-1.47 \cdot 10^{-1}$ \\
Dm12 block & $2.00 \cdot 10^{-5}$ & $-1.22 \cdot 10^{-1}$ \\
Dm20 block & $2.00 \cdot 10^{-5}$ & $-2.23 \cdot 10^{-1}$ \\
Tm9 block & $2.00 \cdot 10^{-5}$ & $-3.05 \cdot 10^{-1}$ \\
\hline
\end{tabular}

Table 4.2: Statistics for T4 and T5 response amplitude to moving edges under input block: Dm4, Dm12, Dm20, or Tm9.

Shown are difference of means: control minus condition, and corresponding p-values from two-sample two-sided permutation test of mean differences.

\begin{tabular}{lccrr}
\hline & \multicolumn{2}{c}{ ON } & \multicolumn{2}{c}{ OFF } \\
\cline { 2 - 5 } & p-value & Mean diff. & p-value & Mean diff. \\
\hline Dm4 block & $3.48 \cdot 10^{-3}$ & $6.22 \cdot 10^{-2}$ & $2.00 \cdot 10^{-5}$ & $1.54 \cdot 10^{-1}$ \\
Dm12 block & $2.00 \cdot 10^{-5}$ & $-8.83 \cdot 10^{-2}$ & $1.37 \cdot 10^{-2}$ & $4.02 \cdot 10^{-2}$ \\
Dm20 block & $2.00 \cdot 10^{-5}$ & $-1.58 \cdot 10^{-1}$ & $2.00 \cdot 10^{-5}$ & $1.24 \cdot 10^{-1}$ \\
Tm9 block & $2.00 \cdot 10^{-5}$ & $-1.72 \cdot 10^{-1}$ & $2.00 \cdot 10^{-5}$ & $1.62 \cdot 10^{-1}$ \\
\hline
\end{tabular}




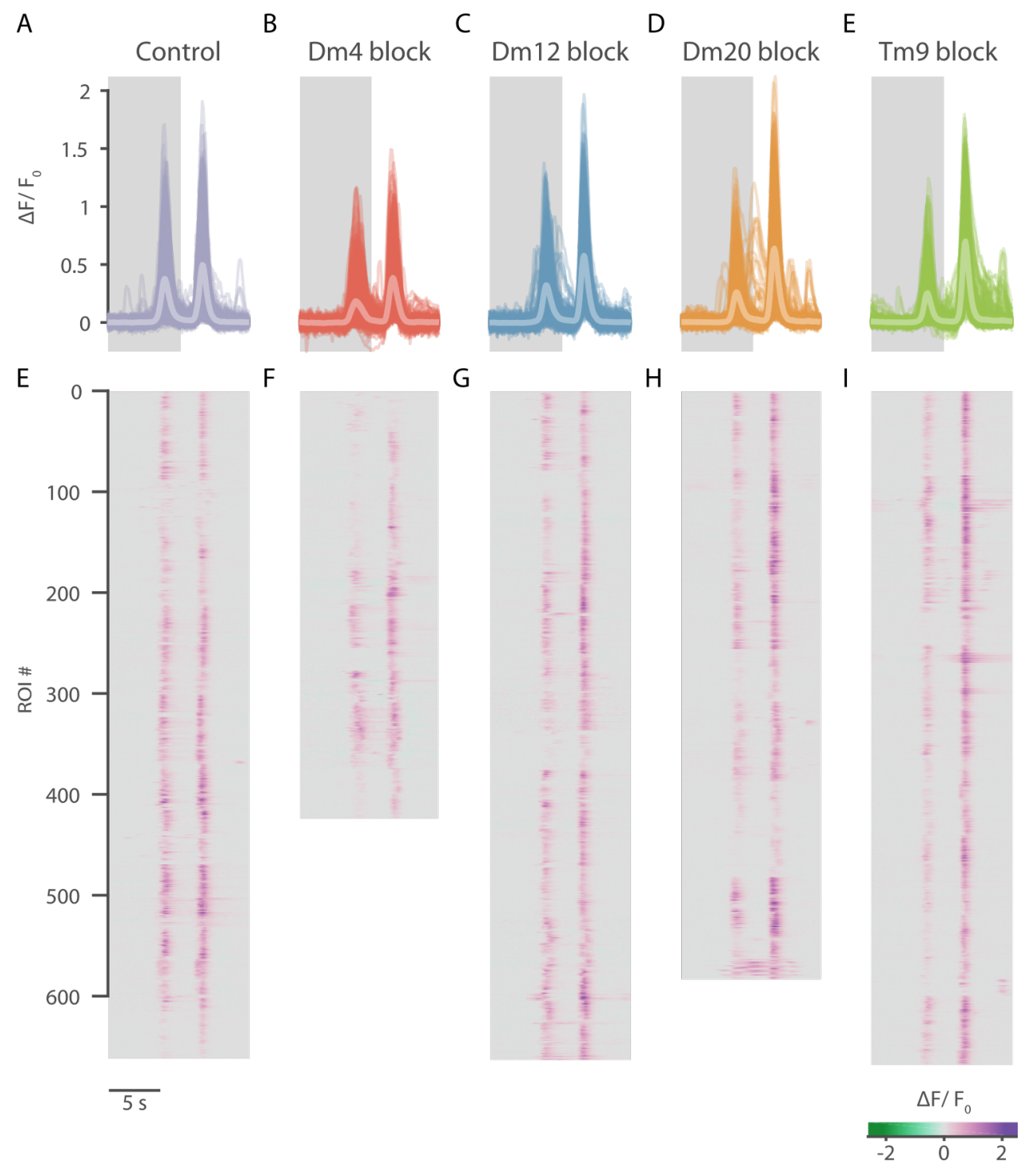

Figure 4.5: T4 and T5 responses to edges upon blocking activity of Dm4, Dm12, Dm20, or Tm9.

A-E. T4 and T5 responses to moving edges for control (A), and block of Dm4 (B), Dm12 (C), Dm20 (D), or Tm9 (E). Every trace is the average response over four motion directions, traces were aligned by maximizing cross correlation before averaging. Mean and standard error are overlaid. F-J. Single response traces stacked for visualization, corresponding to A-E. 
A

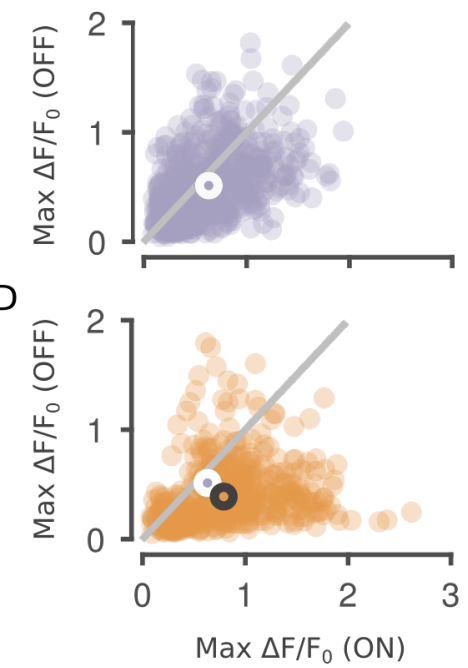

$\mathrm{F}$ Max response to ON edges

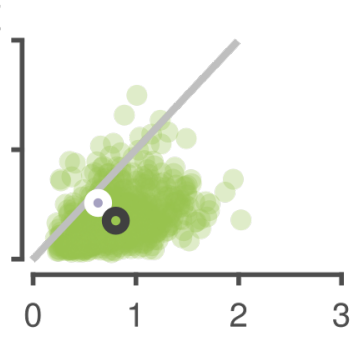

C

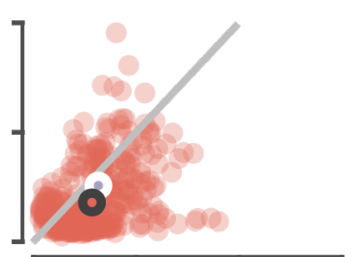

$\mathrm{E}$

G

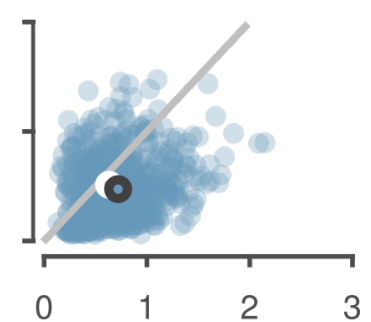

T4/T5 ROI mean

- Control

- Dm4 block

Dm12 block

Dm20 block

Tm9 block
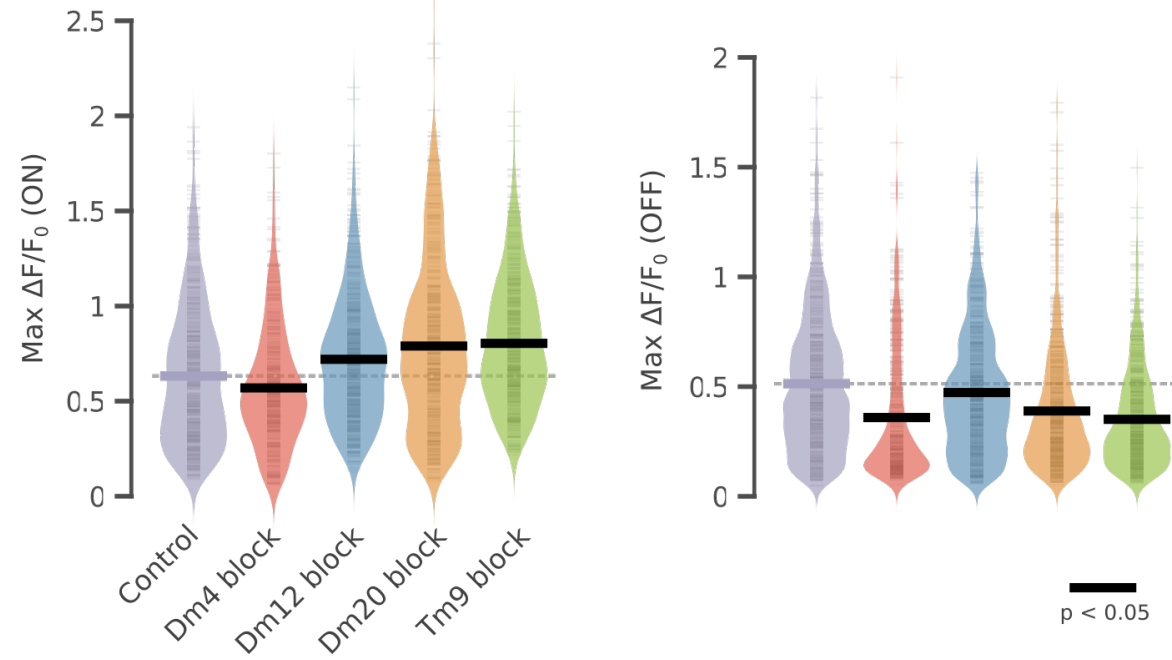

Figure 4.6: T4 and T5 response amplitude to edges upon blocking activity of Dm4, Dm12, Dm20, or Tm9.

T4 and T5 response amplitude to moving edges for control, and block of Dm4, Dm12, Dm20, or Tm9. A-E. T4/T5 maximum responses to OFF vs ON edges across directions for (A) controls, (B) Dm4 block, (C), Dm12 block, (D) Dm20 block, and (E) Tm9 block. F. Distribution of response amplitude to ON edges. G. Distribution of response amplitudes to OFF edges. Blocking Dm12, Dm20, or Tm9 increased responses to ON edges, blocking Dm4 decreased responses to ON edges. Blocking any of the four neuron types increased response amplitudes to moving OFF edges. Statistical tests are summarized in tab. 4.2. 


\section{Preferred directions and direction selectivity}

The main function of T4 and T5 cells is to compute the direction of motion. To quantify how much these neurons respond preferentially to one direction, we used the direction selectivity index (DSI) (eq. 6.12). Neurons responding exclusively to one direction have a direction selectivity index of 1 , while neurons responding equally to all directions have a direction selectivity index of 0 . The preferred direction of a neuron was also calculated (eq. 6.13). We analyzed the responses to ON and OFF separately (fig. 4.7). Interestingly, we observed the preferred direction vectors segregated into four groups, even when the selection of regionsof-interest discarded the information of the origin layer. To visualize better the distribution of preferred directions over flies, we computed the kernel density estimate of preferred directions. Indeed, the distribution of all preferred directions had four lobes corresponding to main groups of preferred directions close to the cardinal directions (fig. 4.8).

The distribution of preferred directions was not the same for ON than for OFF responses, we examined this difference in more detail. Because T4 and T5 neurons were recorded together and they are retinotopically arranged, each region-of-interest should have captured the T4 and T5 axon terminals that look at the same point of visual space. Thus, we asked whether the same direction is encoded by both T4 and T5 neurons at the same location in visual space. Because preferred directions are periodic and have no meaningful start and end point, we calculated the circular correlation of preferred directions for $\mathrm{ON}$ and OFF responses. Because of $\mathrm{T} 4$ and $\mathrm{T} 5$ contrast selectivity, ON and OFF responses are a proxy for T4 and $\mathrm{T} 5$ responses, respectively. Indeed, preferred directions were positively correlated for T4 and T5 neurons in the controls (fig. 4.9 A). However, blocking Dm4 and Tm9 resulted in negative circular correlations of T4 and T5 preferred directions (fig. $4.9 \mathrm{~B}, \mathrm{E}$ ). Thus, Dm4 and Tm9 are needed for normal joint encoding of moving direction of ON and OFF edges at each point in space.

The observed decoupling of preferred directions could be due to a lower direction selectivity that leads to a imprecise estimate of preferred direction. Therefore we next compared the direction selectivity index for T4 and T5 neurons looking at the same point in space. Similarly, the direction selectivity index was correlated for T4 and T5 neurons in controls (fig. 4.9 A), and was negatively correlated for Dm4 block (fig. 4.9 B), and uncorrelated for Tm9 block (fig. 4.9 E). This further supported the idea that Dm4 and Tm9 are required to balance the properties of T4 and T5 neurons. 


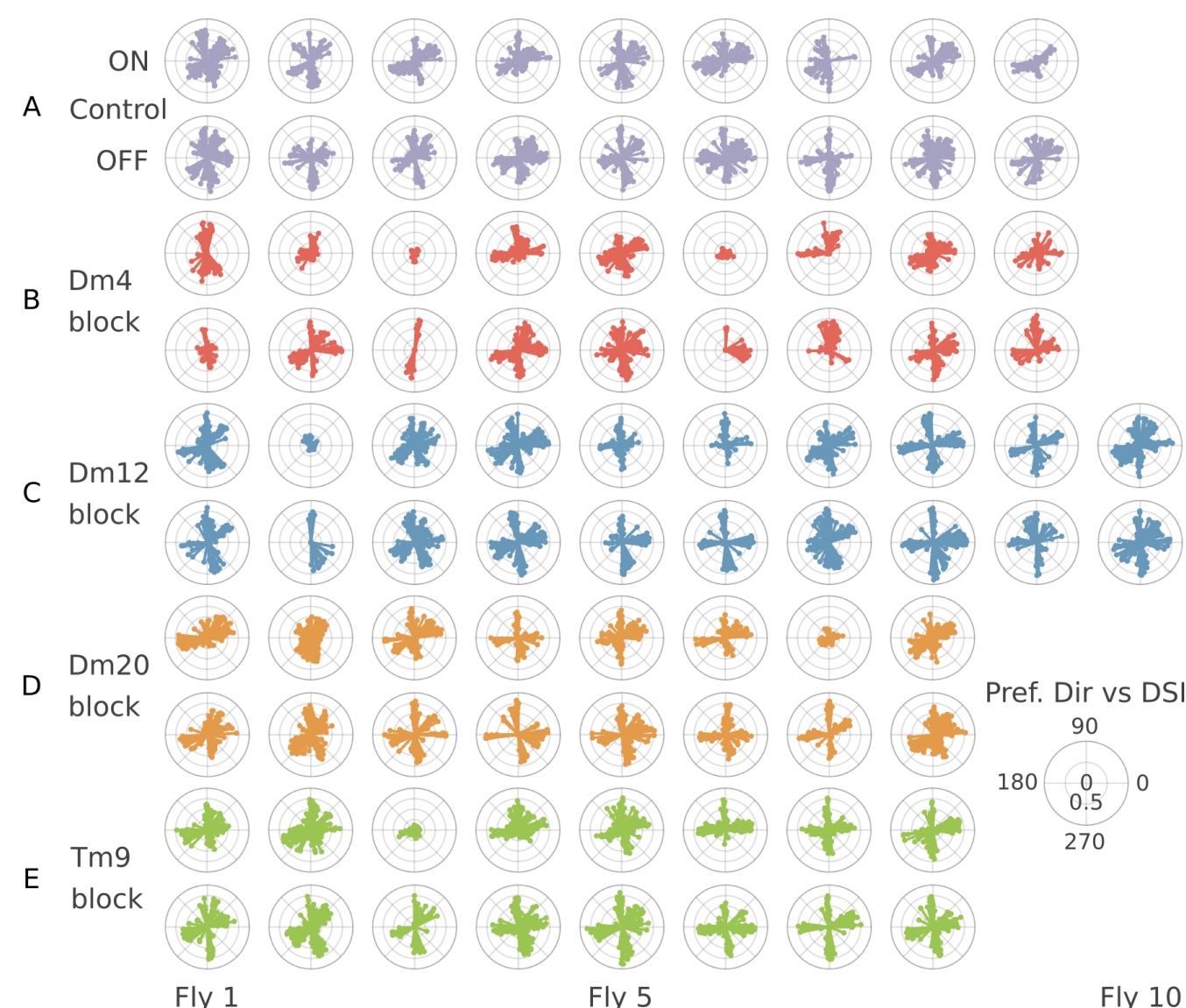

Figure 4.7: T4 and T5 direction selectivity and preferred directions to moving edges per fly, upon blocking activity of Dm4, Dm12, Dm20, or Tm9.

A-E. T4 and T5 direction selectivity vs preferred direction for moving edges, for control (A), and block of Dm4 (B), Dm12 (C), Dm20 (D), or Tm9 (E). Top rows: responses to moving ON edges. Bottom rows: responses to moving OFF edges. Notice that ON responses are impaired while OFF responses remain, in some flies, e.g., fly 7 in Dm20 block, and fly 3 in Tm9 block.

To study the particular effects of input blocks, we quantified the direction selectivity index for ON and OFF edges, equivalent to T4 and T5 responses. Dm4 block decreased T5 direction selectivity index (fig. 4.11 B). Dm12 block increased the direction selectivity index of both T4 and T5 (fig. $4.11 \mathrm{~A}, \mathrm{~B}$ ). Dm20 and Tm9 blocks had similar effects, they increased $\mathrm{T} 4$ direction selectivity index and decreased $\mathrm{T} 5$ direction selectivity index (fig. $4.11 \mathrm{~A}, \mathrm{~B}$ ). Therefore, all wide-field neurons and Tm9 are required 
Distribution of preferred directions for T4 and T5 neurons

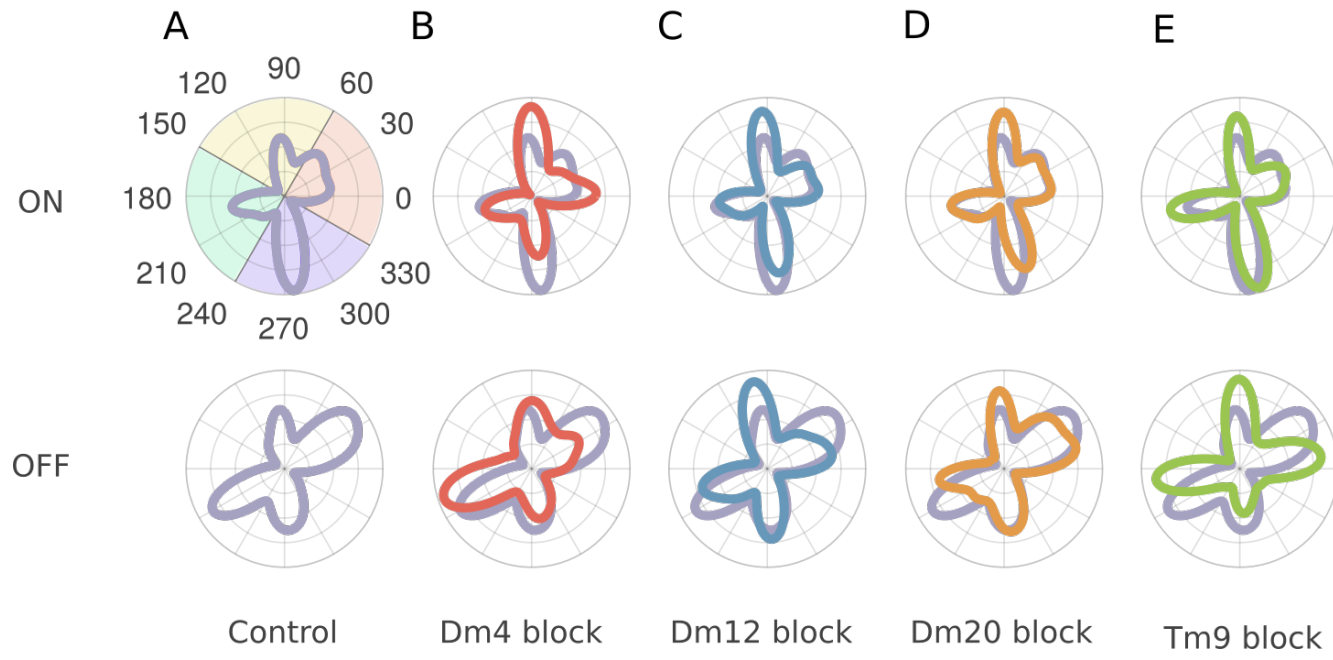

Figure 4.8: Distribution of preferred directions for T4 and T5 neurons upon blocking activity of Dm4, Dm12, Dm20, or Tm9.

A-E. T4 and T5 preferred direction distribution for moving edges, for control (A), and block of Dm4 (B), Dm12 (C), Dm20 (D), or Tm9 (E). Top rows: responses to moving ON edges. Bottom rows: responses to moving OFF edges. The curves are the kernel density estimates of the distributions, note the four lobes. For comparison control distribution is on every block condition. Colored regions in (A) highlight the directions that split the distribution into four sections at the points of lowest density.

for proper direction tuning of $\mathrm{T} 4$ and $\mathrm{T} 5$ neurons.

Because T4 and T5 neurons exist in four subtypes encoding mainly four directions of motion, we asked whether the tested inputs affected equally all four subtypes of T4 and T5 neurons. Thus, we visualized the effects of input blocks over the four main direction groups (as defined in fig. 4.8 A). Indeed, Dm12 block increased tuning in all directions for both T4 and T5 (fig. $4.12 \mathrm{~A}-\mathrm{H}$ ). Also Dm4 block decreased tuning of T5 neurons in all directions (fig. 4.12 E-H ). The effects of Dm20 and Tm9 were similar in three out of the four directions for both T4 and T5. Interestingly, Dm4 block led to opposite effects for opposing directions of motion: increased tuning for upwards and rightwards motion, and decreased tuning for downwards and leftwards motion. This effects canceled each other when grouping over all neurons and directions (fig. 4.11 A). We concluded Dm12, Dm20, and Tm9 have similar effects in all four subtypes of T4 and T5 neurons. Furthermore, Dm4 acts uniformly for all T5 subtypes, but has opposite 
A

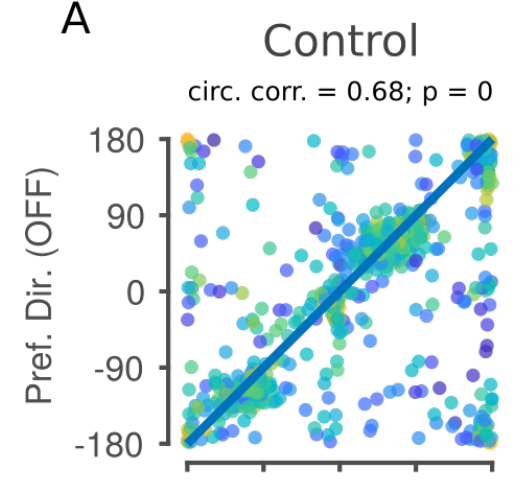

D

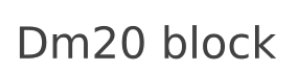
circ. corr. $=0.52 ; p=0$

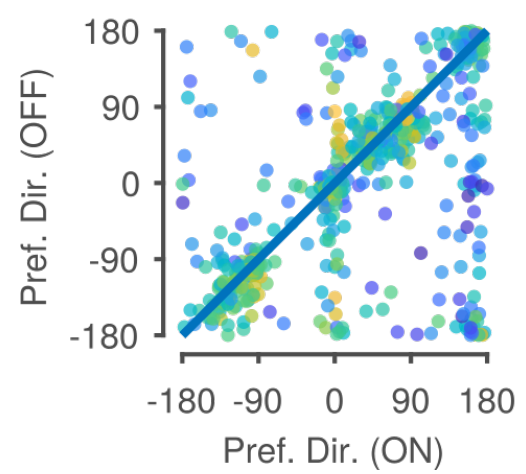

B Dm4 block
circ. corr. $=-0.32 ; p=6 e-11$

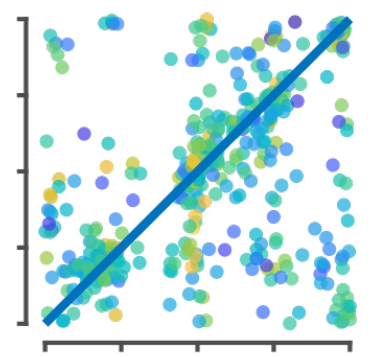

$\mathrm{E}$ Tm9 block
circ. corr. $=-0.11 ; \mathrm{p}=3 \mathrm{e}-3$

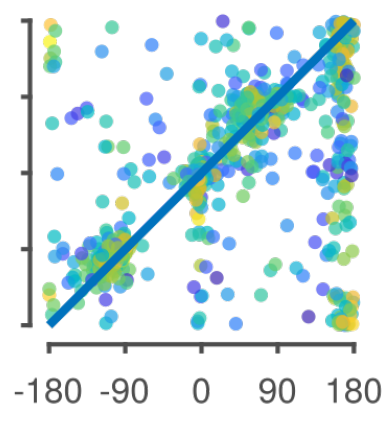

C Dm12 block

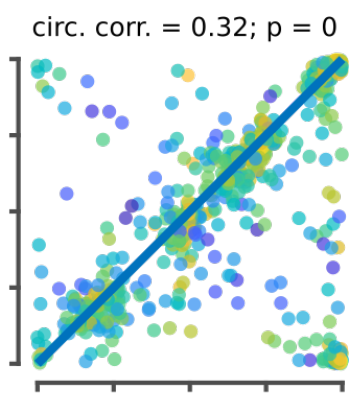

Figure 4.9: Correlation of T4 and T5 preferred directions at the same point in space upon blocking activity of Dm4, Dm12, Dm20, or Tm9. A-E. Circular correlation of T4 and T5 preferred directions for moving edges, for control (A), and block of Dm4 (B), Dm12 (C), Dm20 (D), or Tm9 (E). Color coded is the direction selectivity index. Top: Circular correlation and corresponding p-value. T4 and T5 preferred directions are positively correlated for controls, and Dm12, and Dm20 block, but negatively correlated for Dm4 and Tm9 block.

effects in T4 subtypes encoding opposite directions of motion.

In this section, we provided evidence that multiple wide-field cell types are required for proper scaling of $\mathrm{T} 4$ and $\mathrm{T} 5$ amplitude responses, their directional tuning, and joint encoding of directions for ON and OFF moving stimuli at each retinotopic position. 


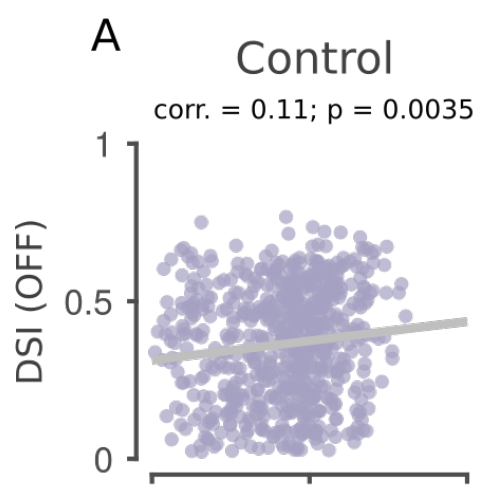

B

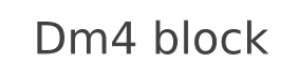

corr. $=-0.22 ; p=5 e-06$

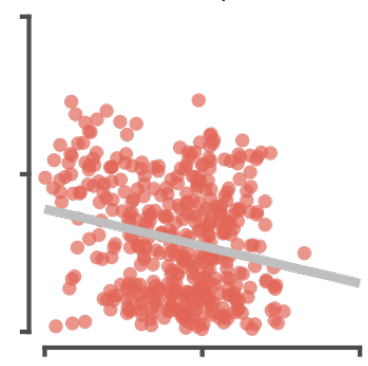

$\mathrm{D}$

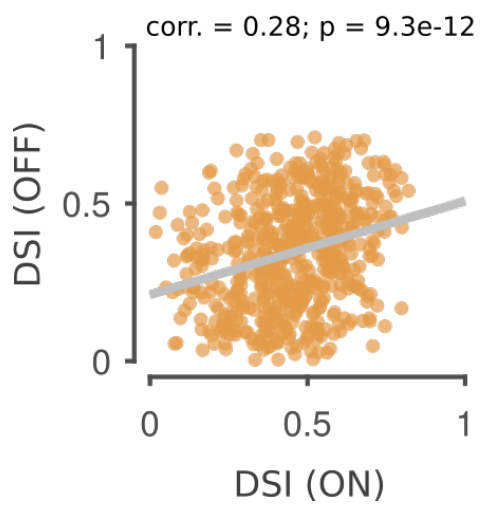

$\mathrm{E}$

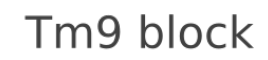

corr. $=0.065 ; p=0.091$

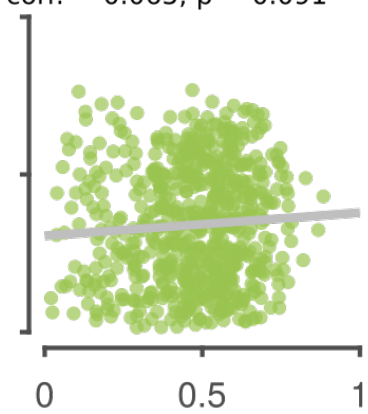

0.5
C

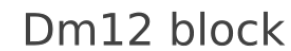

corr. $=0.17 ; p=6.8 \mathrm{e}-06$

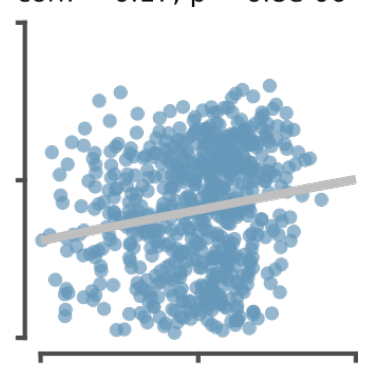

Figure 4.10: Correlation of T4 and T5 direction selectivity index at the same point in space upon blocking activity of Dm4, Dm12, Dm20, or Tm9.

A-E. Pearson correlation of T4 and T5 direction-selectivity indices for moving edges, for control (A), and block of Dm4 (B), Dm12 (C), Dm20 (D), or Tm9 (E). Overlaid is the linear least-squares fit. Top: Pearson correlation and corresponding p-value. T4 and T5 direction-selectivity indices are positively correlated for controls, and Dm12, and Dm20 block, but negatively correlated for Dm4 block, and uncorrelated for Tm9 block. 
A Direction-selectivity index ON

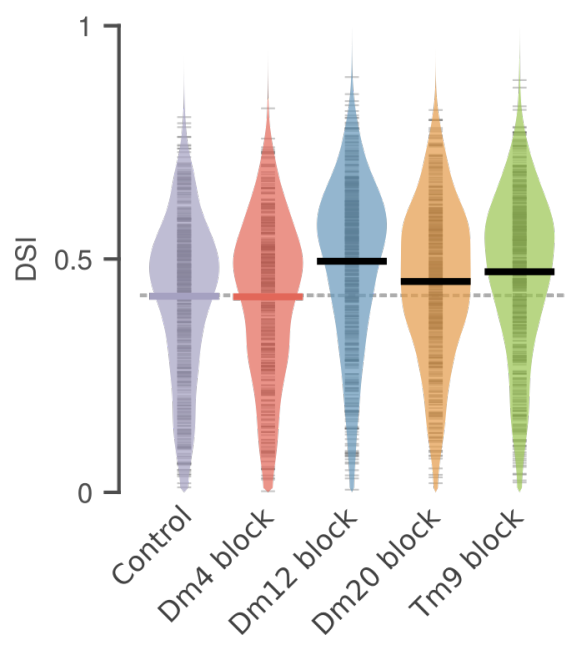

B Direction-selectivity index OFF

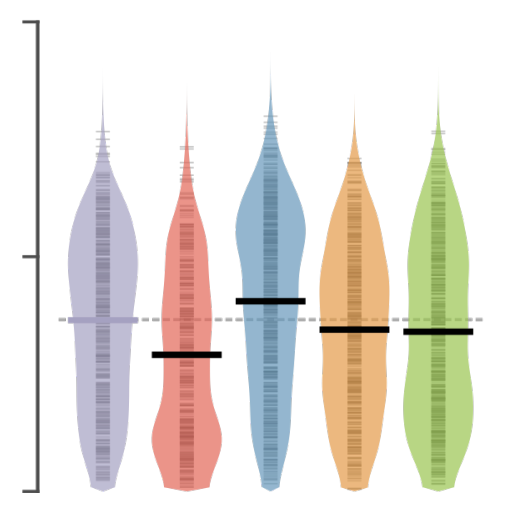

Figure 4.11: T4 and T5 direction selectivity upon blocking activity of Dm4, Dm12, Dm20, or Tm9.

T4 and T5 direction selectivity to moving edges for control, and block of Dm4, Dm12, Dm20, or Tm9. A. DSI for moving ON edges. B. DSI for moving OFF edges. Lack of Dm12, Dm20, and Tm9 increased T4 DSI, lack of Dm12 increased T5 DSI, and lack of Dm4, Dm20, and Tm9 decreased T5 DSI. (Table 4.3)

Table 4.3: Statistics for T4 and T5 direction selectivity to moving edges under input block: Dm4, Dm12, Dm20, or Tm9.

Shown are difference of means: control minus condition, and corresponding p-values from two-sample two-sided permutation test of mean differences.

\begin{tabular}{lrrrrr}
\hline & \multicolumn{2}{c}{ DSI ON } & & \multicolumn{2}{c}{ DSI OFF } \\
\cline { 2 - 3 } \cline { 5 - 6 } & \multicolumn{1}{c}{ p-value } & Mean diff & & p-value & \multicolumn{1}{c}{ Mean diff } \\
\hline Dm4 block & $9.04 \cdot 10^{-1}$ & $1.25 \cdot 10^{-3}$ & & $2.00 \cdot 10^{-5}$ & $7.37 \cdot 10^{-2}$ \\
Dm12 block & $2.00 \cdot 10^{-5}$ & $-7.49 \cdot 10^{-2}$ & & $4.00 \cdot 10^{-5}$ & $-4.04 \cdot 10^{-2}$ \\
Dm20 block & $8.60 \cdot 10^{-4}$ & $-3.16 \cdot 10^{-2}$ & & $4.45 \cdot 10^{-2}$ & $2.01 \cdot 10^{-2}$ \\
Tm9 block & $2.00 \cdot 10^{-5}$ & $-5.24 \cdot 10^{-2}$ & & $1.42 \cdot 10^{-2}$ & $2.45 \cdot 10^{-2}$ \\
\hline
\end{tabular}



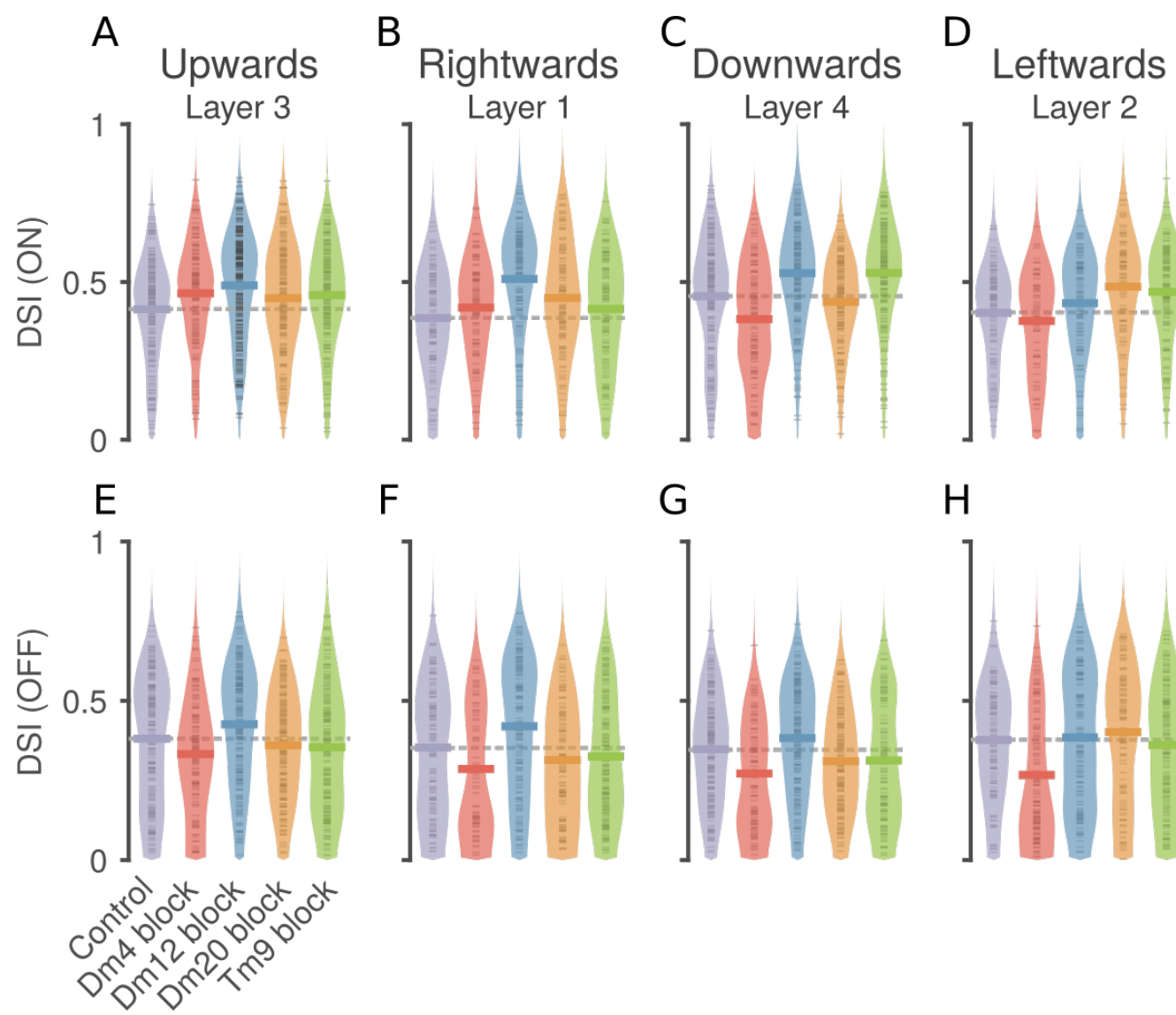

$\mathrm{F}$

G
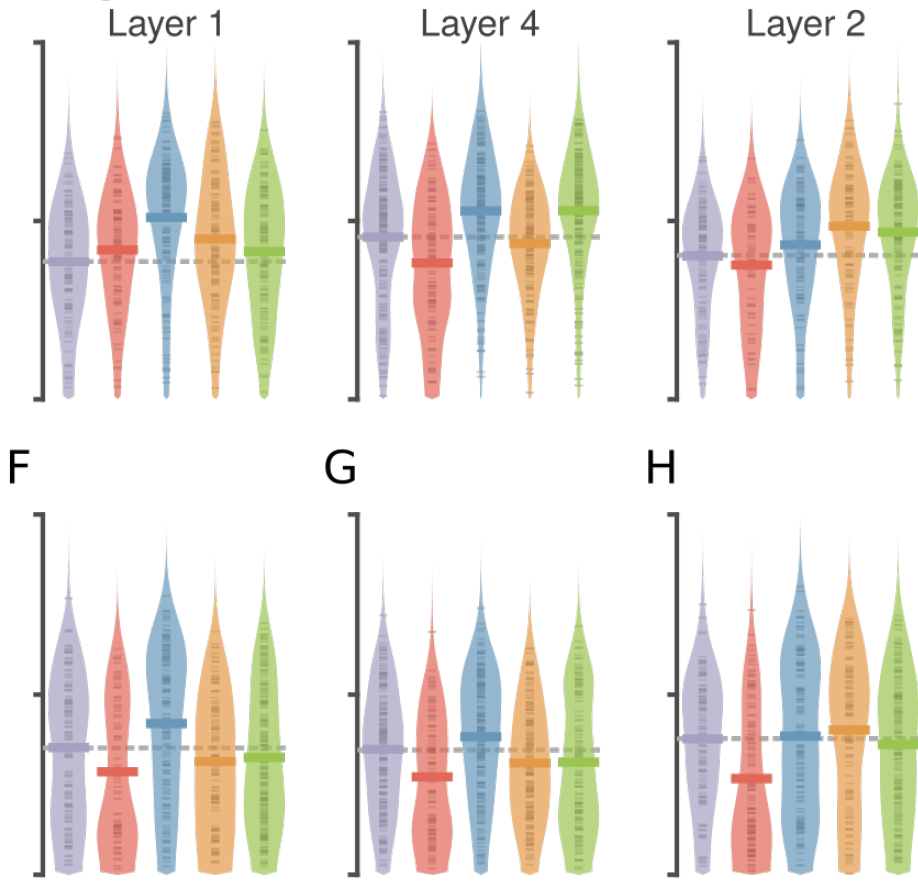

$\mathrm{H}$

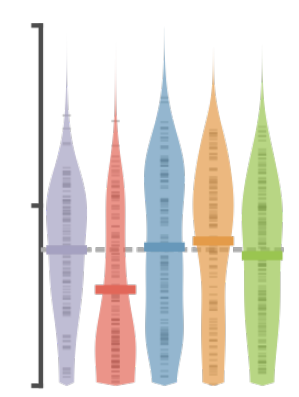

Figure 4.12: T4 and T5 direction selectivity grouped over the cardinal directions upon blocking activity of Dm4, Dm12, Dm20, or Tm9.

T4 and T5 direction selectivity to moving edges for control, and block of Dm4, Dm12, Dm20, or Tm9. A-D. DSI for moving ON edges for upwards (A), rightwards (B), downwards (C), leftwards (D), each direction is mainly represented by one anatomical layer. E-H. Similar to (A-D) but for moving OFF edges. Only Dm12 block had a consistent increase in DSI over all polarities and directions. 


\subsection{Response properties at overlapping T5 dendrites and Tm9 axons}

So far, we demonstrated Tm9 neurons have variable receptive field properties, and they are required for proper responses of T4 and T5 neurons. Thus, Tm9 variability could reflect on some features of T5 responses. To look for relations between T5 neurons and the corresponding Tm9 input neurons, we performed dual in vivo two-photon calcium imaging of T5 neurons expressing the green-emitting GCaMP6f sensor, and Tm9 neurons expressing the red-emitting jRGECO1a sensor (similar to sec. 2.2). We recorded responses to $\mathrm{ON}$ and $\mathrm{OFF}$ bars, and analyzed responses to manually selected regions-of-interest encircling single Tm9 axons terminals. As in previous sections, we fitted a single Gaussian to the tuning curves of T5 and Tm9 neurites within the same region-of-interest.

Spatial receptive fields Both $\mathrm{T} 5$ and $\mathrm{Tm} 9$ neurons responded positively to OFF bars. Tm9 neurons responded with the usual negative response in the center, accompanied occasionally by a positive surround response. Contrarily, T5 did not respond to ON bars, but only to the offset from ON bar to dark background (data not shown). Thus, we started analyzing the relationship of response parameters to OFF stimuli, namely the amplitude, position, and width of the fitted Gaussians. All parameters correlated for both horizontal and vertical bars (fig. 4.13). The tight relative position of T5 and Tm9 receptive fields (fig. $4.13 \mathrm{~B}, \mathrm{E}$ ) is consistent with the evidence from connectomics that $\mathrm{T} 5$ receives inputs from the Tm9 neuron in the same column (Shinomiya, Huang, et al., 2019). Furthermore, T5 dendrites had larger receptive fields than Tm9 axons (fig. $4.13 \mathrm{C}, \mathrm{F}$ ), this is consistent with T5 receiving OFF inputs from Tm1, Tm2 and Tm4 from a neighboring column (Shinomiya, Huang, et al., 2019). About amplitude, we observed two coupling modes, one where T5 and Tm9 amplitudes laid close to the identity line, and other where T5 had larger amplitude than Tm9, irrespective of fit quality (fig. 4.13 A, D).

Spatiotemporal tuning maps We next asked whether the correlation of Tm9 and T5 properties would extend to the spatiotemporal domain. Thus, we probed T5 and Tm9 responses to drifting sine gratings of of different temporal and spatial frequencies, from $0.25 \mathrm{~Hz}$ to $16 \mathrm{~Hz}$ and $0.03 \frac{1}{\circ}$ to $0.50 \frac{1}{\circ}$, respectively. Because we are recording T5 dendrites, we have

\footnotetext{
$2 \frac{1}{\circ}$ is cycles per degree, a dimensionless unit.
} 
OFF receptive field properties of T5 dendrites and Tm9 axon terminals
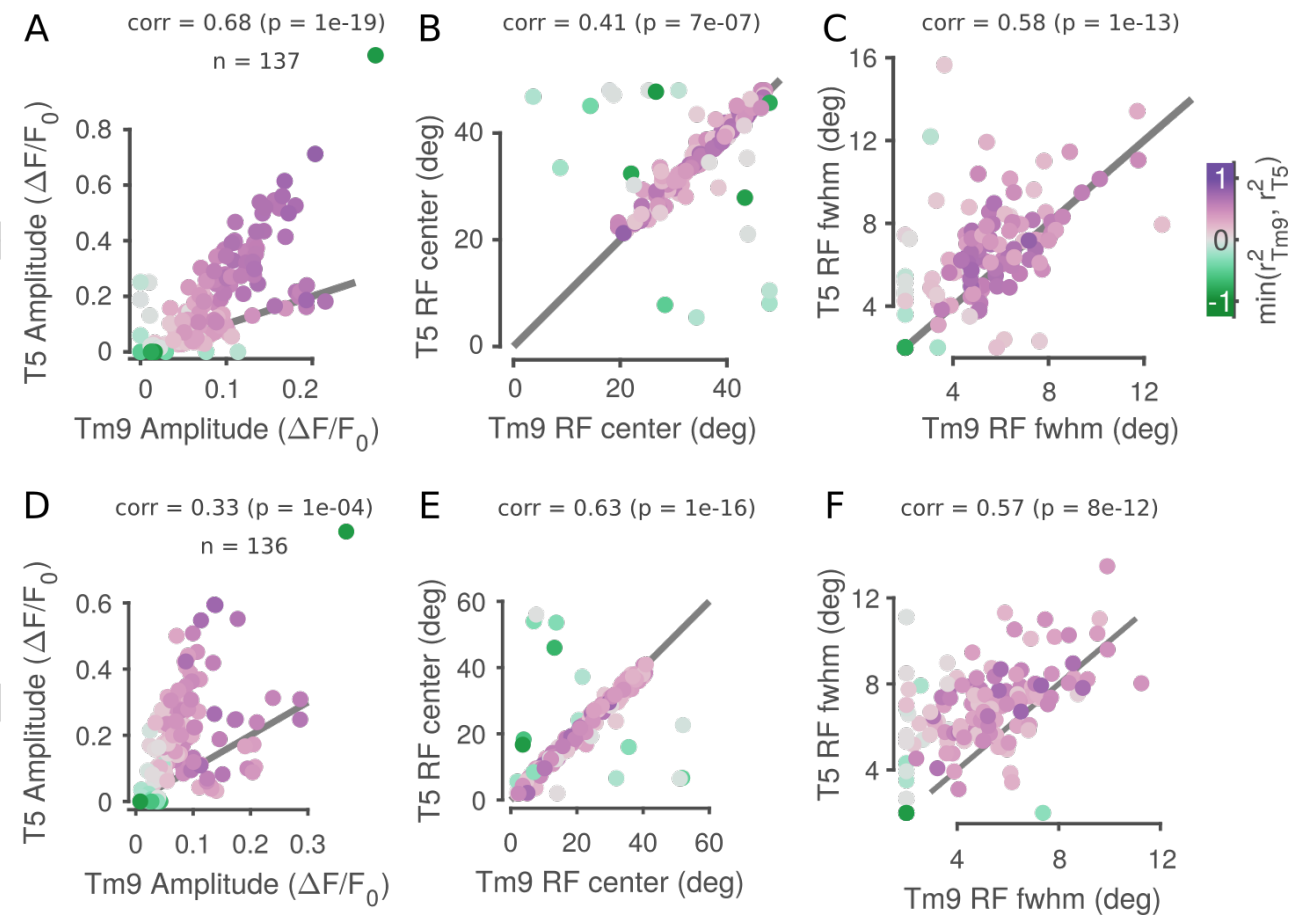

Figure 4.13: T5 and Tm9 spatial OFF response properties are correlated.

Response properties extracted from Gaussian fits to spatial tuning curves to OFF bars. A-C. Response properties for horizontal bars: (A) response amplitudes, (B) receptive field center position, and (C) receptive field width. Pearson correlation between T5 and Tm9 properties and corresponding p-values are on top. D-F. Similarly to (A-C) but for vertical bars. All parameters are correlated for both orientations. Notice amplitudes split along two lines, one close to the identity and other in a line with higher T5 than Tm9 amplitudes. The minimum coefficient of determination $r^{2}$ between T5 and Tm9 fits per neuron was used as a color map. Interestingly, fit quality affected only center positions but not amplitudes or widths.

the dendrites of the four subtypes of T5 neurons overlapping in each eye column. Therefore, we only used sine gratings moving in one direction (leftwards), assuming only the T5 subtype selective to that direction of motion would respond strongly, and the others would respond negligibly. We calculated the spatiotemporal tuning maps using the amplitude of the first harmonic of the response to gratings, obtained via the fast Fourier transform (FFT). The first harmonic was the amplitude spectrum at the 
temporal frequency of the drifting gratings. We calculated the tuning maps for each T5 and Tm9 pair at each region-of-interest. We smoothed the maps using cubic splines interpolation and then averaged them across regions-of-interest. Tm9 axons and $\mathrm{T} 5$ dendrites showed similar mean spatiotemporal tuning maps (fig. 4.14 A, B). To visualize the spatial and temporal frequency tuning curves separately, we split the data at the map peak, along the corresponding axis. About their spatial tuning, both T5 and $\mathrm{Tm} 9$ preferred the largest grating used that had a period of $32^{\circ}$ (fig. $4.14 \mathrm{C}$ ). It is possible both neurons prefer eve wider gratings, but displaying wider gratings was not possible due to the screen size of $60^{\circ}$. Moreover, both neurons had a sharp decline in responses for gratings with period about $8^{\circ}$, consistent with the resolution of one eye column. About their temporal tuning, both Tm9 and T5 preferred $1 \mathrm{~Hz}$ (fig. $4.14 \mathrm{D}$ ), resembling T5 tuning in (Maisak et al., 2013). This is interesting because Tm9 expressed a slower calcium sensor, jRGECO1a, than GCaMP6f expressed by T5, which could influence the temporal tuning comparisons. To exclude parametric comparisons, we simply calculated the correlation coefficient between pairs of T5 dendrites and Tm9 axons at each region-of-interest. Most neuron pairs had large positive correlations, about 0.7 on average (fig. $4.14 \mathrm{E}$ )). Therefore, our data suggested $\mathrm{T} 5$ and $\mathrm{Tm} 9$ properties are correlated in both the spatial and temporal domains. 

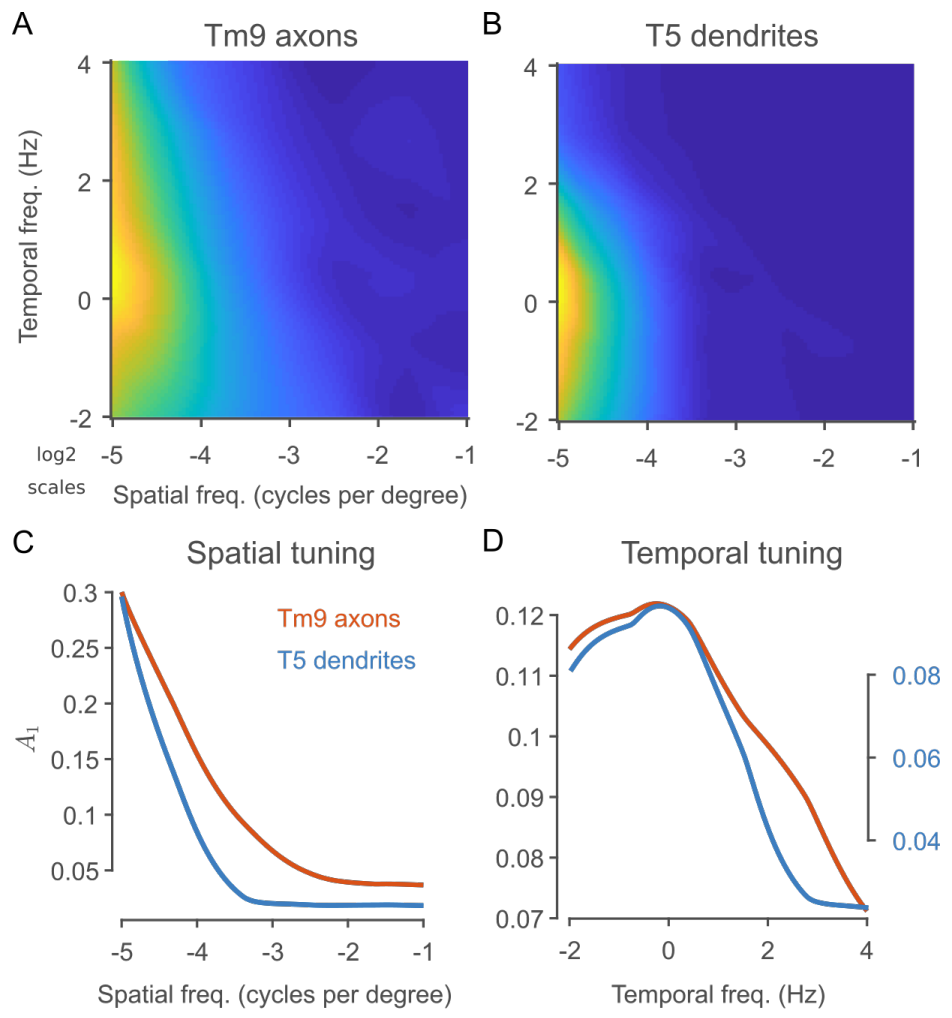

$\mathrm{E}$

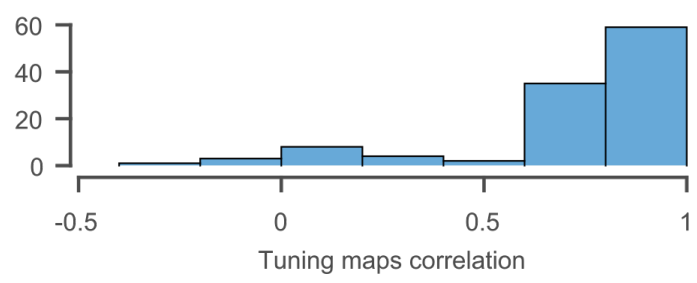

Figure 4.14: Spatiotemporal tuning maps of T5 dendrites and overlapping Tm9 axons are correlated.

A, B. Mean spatiotemporal tuning maps (from drifting sine gratings) of (A) Tm9 axons and (B) overlapping T5 dendrites. Maps were interpolated by cubic splines and averaged for 112 ROIs from 11 flies. All axes for spatial and temporal frequencies are in a logarithmic scale of base 2, e.g., 0 is $2^{0}=1$. Color map shows the first harmonic amplitude from fast Fourier transform (FFT) of the responses to sine gratings, blue (darkest) is lowest amplitude, yellow (brightest) is highest amplitude. C. Slice along the spatial frequency axis for the peak in (A, B), blue is T5 dendrites, red is Tm9 axons. Both neurons preferred the widest gratings (lowest spatial frequency) of $32^{\circ}$ period. D. Similar as (C) but for the temporal frequency. Both T5 and Tm9 peaked at about $1 \mathrm{~Hz}$. E. Histogram of Pearson's correlations between tuning maps of $\mathrm{T} 5$ and Tm9 ROIs, average correlation 0.7 . 


\section{Discussion}

\subsection{Cell types with variable functional properties}

In this thesis, we characterized the receptive field properties and their variability of neurons involved in motion computation. We showed that $\mathrm{Tm} 9$, an anatomically and genetically defined cell type, can display narrow and wide receptive fields.

Our data about $\mathrm{Tm} 9$ receptive field variability reconciles contradictory literature (Fisher, Leong, et al., 2015; Serbe et al., 2016; Arenz et al., 2017). Recording from a population of Tm9 neurons revealed the whole range of FWHMs observed in those references. The variability is observed in the spatial and temporal domains using different stimuli, extending the ones used in those studies: full-field flashes, flashing bars, and noise stimuli. Here, we first want to discuss some assumptions used in fly vision research that led to such contradictions. Given the wonderful regularity of the fly eye, it is often assumed that neuron properties are the same throughout the whole eye. Moreover, studies depend on the genetic background of the flies, thus, the fly and not the neuron is the sampling unit from the underlying genetic distribution. Consequently, in practice fewer neurons are recorded per fly, to allow collecting data from more flies. This assumption would not reveal inhomogeneities unless experimental variations occur unintentionally and lead to observing otherwise ignored brain regions. This approach however has provided most of the findings referenced by this thesis. In fact, there were solid reasons to assume $\operatorname{Tm} 9$, and other neuron types, are functionally homogeneous. Tm9 is an anatomical neuron type, it has a stereotypic shape within the eye and across eyes of different flies that distinguish it from others (Fischbach and Dittrich, 1989), and it is labeled by a genetic line (used here and 
also in (Fisher, Leong, et al., 2015)) that produces the same expression pattern over and over in different flies. Therefore, it is remarkable that Tm9 exhibits variable functional properties defying the homogeneity assumption supported both anatomically and genetically (Davis et al., 2019).

Heterogeneity has long been a part of neuroscience research. Variations exist that are intrinsic to biology and also extrinsic related to measurement errors. Initial measurements will always provide a rough picture, that will be resolved better with ever finer measurement techniques. For example, going from a reticular theory to the neuron doctrine required measurements first by light microscopy and ultimately by electron microscopy to demonstrate the existence of synapses. Sometimes, the biological differences are remarkable and allow robust classifications such as anatomically defined cell types (Ramon y Cajal and Sánchez, 1915; Llinás, 2003). Neuron types defined this way can remain as such until finer measurements reveal previously unattainable details. For example in the vertebrate retina, the number of cell types increased with more advanced studies (Wässle, 2004; Sanes and Masland, 2015; Zeng and Sanes, 2017). Similarly, current studies in the hippocampus, a brain region involved in navigation and memory, highlight the heterogeneity within classical cell types (Cembrowski and Spruston, 2019), interestingly both developments were facilitated by advances in transcriptomics. For the hippocampus, neuronal subtypes were defined for pyramidal neurons on the basis of joint anatomical, genetic, and physiological properties, in agreement with a continuous variation rather than discrete subtypes (Cembrowski and Menon, 2018; Cembrowski, Phillips, et al., 2018). This results highlight even more our findings, because pyramidal neurons vary along all axes, but Tm9 varies only along the functional axis. There are no available reports on heterogeneity in Tm9 regarding gene expression patterns (Davie et al., 2018; Davis et al., 2019; Konstantinides et al., 2018), nor anatomical neuron reconstructions (Takemura, Xu, et al., 2015). However, future studies may reveal the fine gene expression or connectivity variations that could determine the various Tm9 functional properties.

\section{Wide-field inhibitory neurons provide flexible control of receptive field properties}

We went one step down to reveal connectivity variations of Tm9 neurons. We showed wide-field neurons functionally connect to Tm9. These neurons were distal medulla neurons Dm4, Dm12, and Dm20 (fig. 3.7 C). All of them were inhibitory to Tm9 when optogenetically activated. Strikingly, 
Dm4 was excitatory to some Tm9 neurons. Moreover, we showed Dm4 neurons have a heterogeneous presynaptic site distribution (fig. 3.3, 3.4). Also, staining for GABAergic neurons revealed again a heterogeneous distribution in Dm4, resembling the presynaptic labeling results (unpublished, Cagatay Aydin). The inhomogeneous distributions seem to stem from whole Dm4 neurons having or lacking expression of the genes of interest rather than inhomogeneities within single Dm4 neurons. The inhomogeneous distribution of GABAergic Dm4 neurons is consistent with functional connectivity data from optogenetics experiments. A subset of inhibitory Dm4 neurons could decrease Tm9 responses, and the other subset would have to increase Tm9 responses via a excitatory transmission. We further showed Dm4 connects directly to Tm9 using GRASP. A careful analysis of new GRASP experiments could reveal whether connections occur homogeneously in the brain or only subsets of Dm4 neurons connect to Tm9. Future experiments will image both Dm4 and Tm9 neuron responses simultaneously to reveal the coupling of their response properties. To this end we will tag Dm4 neurons with a presynaptic marker to be able to group $\mathrm{Tm} 9$ responses accordingly and potentially link Dm4 to Tm9 heterogeneity.

To quantify variability across different conditions, we used the coefficient of variation (standard deviation divided by mean). We observed that Dm4 has the potential to reduce the coefficient of variation of Tm9 ON receptive field size distribution (fig. $3.4 \mathrm{C}, \mathrm{D}, \mathrm{G}, \mathrm{H}, \mathrm{K}, \mathrm{L}$ ), by reducing the variance or increasing the mean. Dm12 could similarly modulate the variability of Tm9, however Dm4 and Dm12 effects were not statistically significant. Contrarily, Dm20 block increased significantly the variance of the sizes of the ON receptive fields. Thus Dm20 is necessary to reduce the variability in Tm9 properties. Higher sample sizes will give definitive proof of the influence of wide-field neurons into Tm9 responses.

Our anatomical and optogenetic studies of Dm4 support our hypothesis that wide-field neurons can flexibly regulate connectivity and shape response properties of involved cell types. Following this logic, we expect to find variability in the other neurons types receiving inputs from Dm4, and maybe to a lesser extent from Dm12 and Dm20. The amount of this variability would depend on the synaptic weights relative to other input neurons, which we discuss further down.

\section{Heterogeneity within a functional map}

All previous facts posit Dm4 as an intriguing neuron type, with the possibility of having completely different effects downstream. If Dm4 neurons are functionally homogeneous, the same feature of visual space will be 
encoded throughout the brain, but this feature will suppress responses in regions downstream GABAergic Dm4 neurons and it will excite responses elsewhere. To this point we are not aware of any neuron type with such processing mechanism. Because of the heterogeneous functional implications of Dm4 responses, it is important to characterize the features encoded by Dm4 neurons. Here, we showed Dm4 neurons process information locally, each dendritic bulb within one column has a receptive field size matching one column (fig. 4.2). Roughly, Dm4 neurons encode OFF stimuli in their positive responses, and ON stimuli less so in their negative responses. Dm4 neurons are sustained and have slow rise and decay kinetics (fig. 4.1). This provided to our knowledge the first known functional characterization of distal medulla neurons. We hypothesized Dm4 dendritic bulbs have very localized responses by the nature of their long convoluted neurites. Our hypothesis was confirmed by a recent study of another neuron with similar dendritic structure to Dm4, namely the CT1 neuron, which was shown to have very localized receptive fields electrically isolated from each other (Meier and Borst, 2019). There is one CT1 neuron per optic lobe, and the projections cover the whole medulla layer 10 , and the whole lobula layer 1 , the layers where T4 and T5 dendrites are located. CT1 synapses into both T4 and T5 neurons and is thought to be provide them with inhibition (GABAergic) (Shinomiya, Huang, et al., 2019). Thus CT1 functions as many parallel units throughout the brain, while Dm4 functions as parallel units with two possible functional consequences. We believe, studying Dm4 will bring more insights into structure-function relation of neurons and or dendrites in particular. The role of Dm4 and CT1 could be analog, Dm4 acting at earlier stages, and CT1 at later ones. Other anatomically analog cell types in medulla and lamina are also hypothesized to underlie similar circuit motifs with analogous functions in different brain regions (Davis et al., 2019). The role of CT1 in the responses of T4 and T5 neurons is unknown. Here, we showed Dm4 neurons are required for directionselectivity in T5 neurons (fig. 4.11). Moreover, the effects of silencing Dm4 appeared more variable across flies than the other conditions (fig. 4.7). Further experiments are needed to check the role of Dm4 across different stimuli and also at the level of behavior.

To investigate further the heterogeneous map encoded by Dm4, we propose immunostaining of Dm4 neurons with selected markers, to label the whole eye and analyze the statistical spatial structure of the labeling, for example the nearest neighbor distribution of labeled vs unlabeled Dm4 dendrites over eye columns. We must mention, Dm4 neurons tile the fly eye without obvious overlaps (Nern, Pfeiffer, and Rubin, 2015). In our eyes, we observed no obvious pattern, but the suggested experiments 
and analysis would provide a more rigorous test. The markers of Dm4 heterogeneity should then be used in physiological imaging studies to finally obtain a topographical Dm4 functional map.

Similarly, we tried looking for heterogeneity in the functional map of Tm9 and T5 neurons. Our results showed, T5 and Tm9 spatiotemporal properties are correlated (fig. 4.14). However, we did not provide the analysis (in progress) of the T5 properties relative to the size of the Tm9 ON receptive field its most variable property, the functional consequences of this correlations for motion detection will be discussed at a later point. Topographical mapping of T5 and Tm9 responses could further shed light on the encoding of natural scene statistics. We often observed layers of mixed selectivity in out T4 and T5 neuron recordings, however, as an example we used a fly with four selective layers, except for lower portion of first layer, which was mostly leftwards selective but had some more downwards selective neurons (fig. 4.14 C, D). Even without the visual maps, the mixed selectivity was reflected in the wider distribution of preferred directions in some directions over others (fig. 4.7, 4.8). Interestingly, this mixed selectivity tended to be more pronounced for the first layer, hinting at a potential topographical organization. Because all axons targeting layers two to four have to travel through layer one, mixed selectivity can arise by imaging combinations of axons from all layers in different proportions. Moreover, mixed selectivity seemed to localize to lower portions of layer one, thus, the optical sectioning of the lobula plate could further influence the observed selectivity. Topographic origins of mixed selectivity could then be confirmed using sparse genetic labeling to image single neurons in the first layer of the lobula plate to discard mixing signals across neuron subtypes, and imaging of different optical sections at different angles, to discard effects of lobula plate topology. The topographical organization could reflect underlying regularities in natural images. Although there is evidence for an ON-OFF processing bias in visual systems across multiple species that is related to the ON-OFF bias in the content of natural images (Cooper and Norcia, 2015; Chichilnisky and Kalmar, 2002; Leonhardt, Ammer, et al., 2016), it is not clear up to what extent other known spatial biases (topographic organizations) in visual processing propagate downstream in the circuits. Of course, we humans have a region of high acuity vision, the fovea, however that is a continuous map rather than a discrete, random one. On the other hand, photoreceptor opsin expression in primates is random and its study could shed light into the relevance of topographic functional maps.

Similarly, in the fly, there is another substrate for heterogeneity, namely the pale and yellow cartridges defined by the rhodopsin expression in R7 
and R8 photoreceptors. They form the basis of color opponency (UV-green, and UV-blue) in the fly eye (Schnaitmann et al., 2018). Interestingly, flies collected in the wild have a varying ratio of pale to yellow photoreceptors and this variation is reflected in their innate color preferences (Anderson et al., 2017). Thus the ratio (if not the topography) of pale and yellow cartridges can propagate up to behavior. Moreover, the color pathway can provide a link between wide-field neurons and topographical maps. Wide-field Dm8 neurons are required for proper UV preferences in flies, and they integrate signals from about 16 R7 photoreceptors (Karuppudurai et al., 2014). The topographic organization of color vision thus seems to stem from pooling of randomly distributed feature channels. However, the exact distribution of R7 inputs from pale and yellow photoreceptors pooled by a single Dm8 neuron is not known. Dm8 neurons could create a uniform representation by averaging out the spatial variability in wavelength preferences of its many inputs. Alternatively, Dm8 could create another heterogeneous map if each Dm8 neuron were to pool different proportions of R7 pale and yellow photoreceptors acquiring slightly different spectral preference to its neighbors. This hypotheses rely on the assumption that Dm8 actually pool signals over their dendritic fields, which has yet to be tested, because we have evidence that even neurons with wider dendritic fields like Dm4 and CT1 can act only locally. We showed that Tm9 receives inhibition from R8 photoreceptors (fig. 3.7 A). Therefore the topographic variability of $\mathrm{R} 8$ spectral preferences is inherited by $\mathrm{Tm} 9$ responses. It would be interesting to test whether both pale and yellow photoreceptors synapse to Tm9, and if so if they do it with similar weights. Assuming Tm9 receives one to one inputs from both types of $\mathrm{R} 8$ photoreceptors, we can expect some spatial heterogeneity in $\operatorname{Tm} 9$ spectral preferences. However, Tm9 not only integrates signals from R8, but from various other cell types, which could reduce the net effects of R8 variability on Tm9 response properties. Thus, studying dendritic integration is important to understand to what extent features are conserved or transformed as information flows along neural circuits.

\section{Multiple cell type convergence into Tm9: robustness or multi-feature integration?}

In this thesis we demonstrated Tm9 receives diverse functional inputs from multiple cell types connected directly or indirectly to Tm9 (fig. 3.7). From connectomics, we inferred that Tm9 inputs are distributed more uniformly over cell types than those of other neurons in the OFF motion pathway 
(fig. 3.1). This degree of cell-type input convergence is higher than for the connectivity between cell types of the mammalian retina (Masland, 2012). For example, connectivity maps approximated from contact areas of electron microscopy reconstructions of a portion of the mouse retina revealed synaptic circuits involving up to nine cell types, however contact area does not imply synaptic connectivity, and therefore this would likely be an overestimate of connectivity. Indeed, Peter's rule, which predicts larger connectivity for larger contact area, is usually violated in both the mammalian retina and the fly optic lobes (Helmstaedter et al., 2013; Takemura, Xu, et al., 2015). Further evidence about large convergence of cell types onto Tm9 was provided by our silencing experiments. No single cell-type block completely abolished responses of Tm9 neurons, and block of wide-field inputs even enhanced responses because of their inhibitory nature to Tm9 (sec. 3.2). Even blocking two inputs at once did not completely silenced Tm9 responses to full-field flashes (Fisher, Leong, et al., 2015). Assuming each input cell type to Tm9 encodes a different feature of the environment, Tm9 integration across multiple cell types would result in Tm9 selectivity to a more complex feature. Therefore, discovering the particular feature selectivity of Tm9 will require experiments using different stimuli across wide range of conditions. Only then, we could probe the effects of input silencing into Tm9 selectivity. Alternatively, Tm9 could multiplex the inputs to provide information to parallel pathways besides motion detection. For example, neurons types in the lobula are known to be feature selective and project to the central brain, for example the lobula columnar neuron LC11 is selective to moving objects (Keleş and Frye, 2017), and LC10 encodes visual features required for courtship (Ribeiro et al., 2018). Many other features are likely going to be elucidated in the near future (Wu et al., 2016). Because Tm9 projects to the lobula, it is thus likely that Tm9 could participate in multiple pathways leading from the lobula to the central brain by transmitting multiplexed features. We find the study of feature selectivity an exciting area for understanding higher-order computations.

Alternatively, Tm9 function could be restricted to one pathway, namely the motion pathway. At least, direction-selective neurons T5 received the most synapses from Tm9. Thus, the convergence of multiple cell types into Tm9 could provide robustness to ensure proper motion encoding even under missing or defective processing in some cell types. Furthermore, errors in connectivity from additional cell types would be reduced when averaged over all inputs. Such additional connectivity errors do exist in the fly optic lobe (Takemura, $\mathrm{Xu}$, et al., 2015). Supporting the robustness hypothesis is the redundancy in the response properties of Tm9 inputs. 
All L1 to L3, and Dm4, Dm12, and Dm20 respond positively to OFF. Tm9 gets excited by L1 to L3 (and some Dm4 neurons), and inhibited by Dm4, Dm12, and Dm20. Therefore, Tm9 has access to redundant OFF excitation and inhibition from at least three cell types each.

Tm9 receives main inputs from L3 neurons, which are luminance sensitive (Ketkar et al. unpublished). Thus Tm9 should carry some of this information, and potentially integrate more features as explained in the above paragraph. Here, we focused for sake of time into figuring out the wide-field properties of Tm9, rather than trying to modulate its properties with luminance levels. Experiments to test Tm9 contribution to luminance or contrast adaptation can be performed, for example by measuring receptive field properties in several orders of magnitude dimer or brighter mean luminance levels, in wild-type and in combination with L3 silencing. Because some Tm9 neurons were robust to blocking L3 inputs, maybe only probing Tm9 in different luminance regimes will reveal an even stronger phenotype.

Further mechanisms for integration are gap junctions, we showed by RNAi silencing of shakB, that $\mathrm{Tm} 9$ receptive fields have electrical coupling contribution. However this contribution was rather small or restricted to one orientation (fig. 3.21). Moreover, it is not clear what cell types are electrically coupled to Tm9. Transcriptomic data could provide some guidelines as to what other anatomically proximal neurons express the complementary gap junction proteins. Connectomics data used here was restricted to chemical synapses, thus, this does not exclude the possibility that some chemical inputs may be as well electrically couple to Tm9.

In general, how general is this wide convergence of cell types that leads to response robustness or multi-feature integration remains to be tested. A first step would be to characterize the other main inputs to T5: Tm1, Tm2, and $\mathrm{Tm} 4$.

Understanding what information from the world is encoded by a neuron is challenging. Even when considering single cell-type inputs, we could face complex spatiotemporal integration by the receiving neuron. Recent studies aimed at inferring the receptive field substructure of neurons in the retina (Maheswaranathan et al., 2018; Liu et al., 2017; Shi et al., 2019; Freeman et al., 2015), and hierarchical models are also applied to higher visual neurons such as those in cortical areas V2 (Hosoya and Hyvärinen, 2015) and IT (Yamins and DiCarlo, 2016). In particular, deep learning is becoming trending as a neuroscience research tool (Turner et al., 2019). We envision that using sophisticated analysis methods combined with the fine genetic toolbox of Drosophila melanogaster will lead to rapid advances in receptive field dissection. 
Additionally, neural integration can be tested experimentally. For example, we could extend out optogenetic experiments to two independent optical channels, expressing opsins of different spectral preferences in two distinct cell types, while recording another cell type in yet another optical channel. For example we could express Channelrhodopsin-2 (Boyden et al., 2005) in L1 and CsChrismon in Dm4 to study how they are integrated by Tm9. We could also study spatial integration using spatially restricted optogenetic excitation of multiple neurons (of the same type) simultaneously while imaging another neuron (Packer et al., 2015). This way, we could test whether neighboring dendrites from wide-field neurons are integrated by $\operatorname{Tm} 9$, and if so if the integration is linear or nonlinear. Moreover, applying this methods to genetically accessible novel wide-field neurons in the fly could shed light into general mechanisms of dendritic integration.

\section{Role of wide-field inhibitory neurons in early vision in Drosophila}

Functional characterization of wide-field neurons is scarce in the fly visual system, with exception of wide-field neurons in the lobula plate selective for global motion that have received attention for decades (Krapp, B. Hengstenberg, and R. Hengstenberg, 1998). Here, we showed that distal medulla neurons Dm12 and Dm20 sharpen the ON receptive fields of Tm9, and provide inhibition to ON and OFF stimuli. This points at roles in lateral inhibition, consistent with this are the receptive fields measured for Dm12 which are wider for ON than for OFF and both span multiple columns. This organization resembles the classical center-surround structure encountered in the vertebrate retina. Furthermore Dm4, Dm12, and Dm20 neurons are required for the tuning of direction-selective neurons T4 and T5. Likely, the effects will be reflected at the behavioral output (preliminary data, not shown). All of these processing roles are fulfilled by some horizontal or amacrine cell types in the retina (Diamond, 2017). To understand the roles of wide-field neurons in multiple parallel pathways, we need to map their postsynaptic partners. Genetic trans-synaptic labeling methods are available (Talay et al., 2017), thus, this experiments are possible in the immediate future. There are at least twenty types of wide-field neurons in the medulla, only from the distal medulla family. Given the large diversity of this family, it is logical to assume multiple unknown computations in the fly can be revealed by studying the functional roles of distal medulla neurons. Especially because in the vertebrate retina, most computations 
require inhibition originating from amacrine cells (Gollisch and Meister, 2010; Diamond, 2017). Given the genetic access to amacrine-like cells in Drosophila melanogaster, we think they can provide useful insights to guide further research on role of inhibitory amacrine cells in other species.

\subsection{Why variability?}

\section{Is connectivity shaped actively or a result of random variability?}

Connectomic reconstructions from seven columns of the fly eye reveal connection errors below $1 \%$. But within the analyzed neurons, Tm9 seemed to haver larger variations (Takemura, $\mathrm{Xu}$, et al., 2015). Moreover the scaling of mean synapse counts and the respective variance could be approximated by a Poisson distribution, indicating that normal connectivity is itself variable. Wiring variations can influence functional properties of neurons (Tobin, R.I. Wilson, and Lee, 2017). This suggested that even when connection errors are small, $\operatorname{Tm} 9$ could have more flexibility in the distribution of synaptic inputs. Moreover, during development visual neurons including Tm9 exhibit activity patterns that are not depending on visual stimulation and the correlations between activity patterns of different cell types reflect adult connectivity (Akin et al., 2019). In particular, Tm9, L3, and Dm4 are correlated more than other cell types, consistent with our functional and silencing studies of Tm9 response properties. Importantly, flies used for optogenetic experiments were blind, so flies developed without visual stimulation. Nevertheless, functional connectivity remained between Tm9 and Dm4. Moreover the functional connectivity exhibited variations consistent with the heterogeneous presynaptic release site distribution and GABAergic labeling, experiments that were carried in flies with normal vision. Changing environmental conditions during development may provide insights into the robustness of connectivity and variations thereof, in particular using Tm9, L3 and Dm4 as a testbed. The connectivity between these cell types is thought to be regulated by pairs of specific ligand and receptors (Tan et al., 2015), providing a genetic handle to the connectivity in the system.

We must note that the connectomic analysis of Tm9 entirely ignored wide-field neurons. Therefore, the synaptic input distribution of Tm9 could be broader and more variable. Interestingly, the more synapses a neuron makes, the more errors it has in connecting to target cell types in wrong columns, whereas it makes less errors in connecting to wrong cell types 
overall (Takemura, Xu, et al., 2015). With more synapses Tm9 could then reach out to inputs in columns other than its own.

Information about branching variations in (Takemura, Xu, et al., 2015) supports Tm9 anatomical homogeneity:

Our reconstructions reveal minor variations in branching patterns of the same cell type in different columns that we interpret as relational, which are required to match reciprocal variations in target neurons so as to establish constancy in pathway strength

\section{Role of efficiency constraints on fly brain wiring for flexible circuit connectivity}

Evolution constrained fly wiring to be very efficient in terms of energy and materials (Niven and Laughlin, 2008), a proof of this is the location of cell bodies outside of the neuropiles to optimize wiring distances and space usage (Rivera-Alba et al., 2011). If this constraint is the largest for building the Drosophila melanogaster brain, then adding new neuron types to fulfill new functions may be incredibly expensive. Like building a new room for a home studio, instead of re-purposing existing space within the home, using the same space and fewer resources. In the same way, new features can be extracted by combinatorial integration of already existing cell types. In this regard, one neuron can become a flexible switch to regulate new configurations. For example, Dm4 shows heterogeneous functional inputs to Tm9, release sites, and neurotransmitter phenotype. It is possible that only Dm4 is so variable, because varying multiple parameters at once otherwise greatly increases the number of potential outcomes and may complicate convergence towards a useful configuration. On the other hand, silencing Dm12 seems to increase the receptive field size of Tm9 neurons, making the distribution more uniform. This variability control may indicate subtle changes on connectivity, maybe only seen as overall synaptic strength between the connections rather that all or none as in Dm4 case. This can be a form of plasticity existing in multiple neurons in the fly brain since the effects can be finely tuned, rather than switched between two states. Thus, multiple mechanisms may be adaptively regulating brain wiring and function in Drosophila melanogaster, with the stronger mechanism restricted to one or few cell types. While variability offers evolutionary advantages (Hiesinger and Hassan, 2018), it could as well be a deficiency of the fly vision, which may hinder fly behavior or could be tolerated by it. This can be tested by single fly behavior quantification, 
followed by brain dissection and labeling of Dm4 synaptic properties (neurotransmitter, presynaptic release sites, etc.). The variability could be a random phenomenon that will drive natural selection in a way that some flies may be better suited to the potentially changing environment, but this question will require longer time span to be tested than the duration of a $\mathrm{PhD}$.

\section{When to stop sub-dividing cell types}

Morphology is useful as a classification. Here, we see that molecularly, a morphological cell type can show contrasting molecular differences (heterogeneous presynaptic protein distribution and GABAergic marker expression for Dm4). A molecular difference as big as neurotransmitter expression can lead to completely opposite effects downstream, e.g. exciting versus inhibiting the postsynaptic cell. Ultimately this effect can greatly affect the functional heterogeneity of the next anatomical cell types. Because neurons downstream do not need to know about the morphology of the input neuron, but only about the signal (electrical or chemical) they receive from it, we argue that functional classifications should be prioritized. Nevertheless, the division should stop at a level in which we can make sense of the found classes, before we dive into deeper details.

\subsection{What we learned from motion detection}

\section{Implications of the receptive fields of interneurons for models of direction selectivity}

Most receptive fields were measured only with OFF bars or noise bars for calcium imaging. Calcium imaging makes it hard to detect signals below baseline, when the baseline itself is small. Here, I show Tm9 and Tm2 neurons have both ON RFs. Tm 9 and Tm2 neurons projecting to T5 come from neighboring columns (Shinomiya, Huang, et al., 2019). Thus, T5 has access to ON signals from main medulla inputs in both receptive field locations (ON RFs are larger than OFF RFs therefore The ON RF of Tm2 from one column will overlap with the ON RF of Tm9 from neighboring column). This supports linear integration as the mechanism for directionselectivity. Here, we still need to consider response kinetics. We propose our approach of dual imaging of input and output neurons will shed more light into motion computation in the fly. 


\section{Limitations}

Regarding studies of wide-field pathways in OFF motion detection, cell types other than Tm 9 can convey signals in parallel from Dm neurons to T5. As mentioned before, we can map the pathways emerging from Dm neurons with a focus on direction selectivity. Their potential role may just be tuning the response strength and receptive field size. Studying the postsynaptic partners of Dm neurons and their receptive fields, especially under Dm neuron silencing, will prove if this is their general function. Alternatively, the development of synapse specific perturbation methods will enable studies of pathway-specific contributions from Dm neurons through Tm9 to T5 cells. While imaging T5 dendrites we approximated responses to one cell type by using stimuli that would preferentially activate a single T5 subtype. To overcome these assumptions and limitations, sparse genetic expression of a calcium sensor in T5 can lead to single neuron labeling, on the other hand, the data collection will be more labor intensive.

An important limitation of this study is the stimulation screen size. Tm9 receptive fields may be affected by boundary effects, since the screen is smaller relative to the whole fly visual field. However, even if this is the case, the effect observed in $\mathrm{Tm} 9$ is not observed in other neurons, or at the least is exclusive to ON stimulation. Further analysis is needed to study asymmetries in $\mathrm{Tm} 9$ receptive fields, this asymmetry can be influenced the the perspective correction applied to the stimulus projection. However, the fact that some neurons were symmetric and some were not speaks in favor of potential asymmetry in the receptive fields.

\subsection{Thoughts on research methodology}

Many of the next points may seem obvious to the reader, however, they are important lessons learned throughout this thesis work. To reveal and reconcile contradicting reports regarding $\mathrm{Tm} 9$ properties, our work highlights the importance of recording more neurons per fly, without reducing the usual number of flies. This requires more data collection. Although there is always a trade-off of resources dedicated to one experiment and the number of possible experiments and hypotheses to test, we suggest that thorough experiments with solid results be performed once, rather than testing multiple hypothesis with a lower statistical power. We find this important given the perceived reproducibility crisis in contemporary research (Baker, 2016). Another layer that obscures data variability is the data visualization process itself. Typically, during data analysis, different 
trials recorded per neuron are averaged, losing the trial variability. Then the trial averages of neurons are first averaged per fly, and quantification is done using only fly means. The variability within a fly is then lost. Then data is summarized and visualized by the fly mean and the corresponding standard error, without individual data points. This way even the variability across individuals is obscured. We advocate for a efficient visualization of variability in the data, although we are aware that effectively visualizing uncertainty and probability distributions is an active research topic (Kale et al., 2019), and we might have failed to do so in this thesis. Nevertheless, the increasingly complex datasets acquired by current experiments highlight the importance of keeping up to date with the latest effective data visualization tools. Furthermore, more sophisticated hierarchical statistical analyses could provide information of effects across trials, neurons, and individuals, without the need to discard information from lower levels (Good, 1980; Allenby, Rossi, and McCulloch, 2005). Moreover, effective data sharing will allow different groups to perform independent analyses of the data potentially revealing more insights. This is in line with current efforts to foster open science and data sharing (McKiernan et al., 2016; Munafò, Hollands, and Marteau, 2018; Farnham et al., 2017).

\subsection{Summary}

- We showed Tm9 is an anatomically and genetically defined cell type that exhibits functional variations. This reconciled a discrepancy in the literature.

- We characterized new ON receptive field properties of transmedullary inputs to T5, favoring linear summation models, or models than integrate both $\mathrm{ON}$ and OFF responses.

- We identified novel wide-field inputs to Tm9: Dm4, Dm12 and Dm20.

- Tm9 ON receptive field properties are sharpened by Dm12 and Dm20 neurons.

- We showed Dm4 is an anatomical substrate for functional heterogeneity, as reflected by positive and negative optogenetic effects on $\mathrm{Tm} 9$ responses.

- We suggested Dm neurons offer flexible control of functional properties in the Drosophila brain. 
- We demonstrated Tm9 receptive field integrates inputs from multiple cell types and is robust to single input type silencing.

- We characterized receptive fields of Dm neurons, and showed they are required for normal function of direction-selective T4 and T5 neurons.

- We proposed flexible connectivity as a mechanism regulating functional heterogeneity in a morphologically and genetically defined cell type. 



\section{Materials and methods}

\subsection{Fly husbandry}

Flies for imaging and behavior experiments were raised at $25^{\circ} \mathrm{C}$. They were kept in a dark light cycle of $12 \mathrm{~h}$ starting at $8 \mathrm{~h}$ CET. They were grown in plastic vials containing food based on molasses. Flies up to ten days from eclosion were used for optogenetics, and up to five days from eclosion for normal functional imaging.

\subsection{Fly genotypes}

The following genotypes have been used in this thesis.

Table 6.1: Genotypes of experimental lines used in chapter 2.

\begin{tabular}{|c|c|}
\hline Genotype & Used in figs. \\
\hline$\frac{w^{+}}{w^{+}} ; \frac{T m 9-l e x A p 65^{a t t P 40}, \text { lexAop-GCaMP } 6 f^{a t t P 5}}{+} ; \stackrel{+}{+}$ & $2.1-12,16,17$ \\
\hline$\frac{w^{+}}{w^{-}} ; \frac{T m 9-l e x A p 65^{a t t P 40}, \text { lex Aop-GCaMP } 6 f^{a t t P 5}}{\text { Tm4-splitGAL4 }} ; \frac{U A S-j R G E C O 1 a}{\text { Tm4-splitGAL4 }}$ & $2.13-2.15$ \\
\hline$\frac{w^{+}}{w^{-}} ; \frac{T m 1-s p l i t G A L 4}{U A S-G C a M P 6 f} ; \frac{T m 1-s p l i t G A L 4}{+}$ & $2.16,2.17$ \\
\hline$\frac{w^{+}}{w^{-}} ; \frac{T m 2 \text {-splitGAL4 }}{U A S-G C a M P 6 f} ; \frac{\text { Tm2-splitGAL4 }}{+}$ & $2.16,2.17$ \\
\hline$\frac{w^{+}}{w^{-}} ; \frac{T m 4-s p l i t G A L 4}{U A S-G C a M P 6 f} ; \frac{T m 4-s p l i t G A L 4}{+}$ & $2.16,2.17$ \\
\hline
\end{tabular}


Table 6.2: Genotypes of experimental lines used in section 3.1.

\begin{tabular}{|c|c|}
\hline Genotype & Used in figs \\
\hline$\frac{w^{+}}{w^{-}} ; \frac{T m 9-l e x A p 65^{a t t P 40}}{S S 02361(D m 4 \text {-split } G A L 4)} ; \frac{\text { lexAop-GFP } P^{11}, U A S-s y b:: G F P^{1-10}}{S S 02361(D m 4-s p l i t G A L 4)}$ & 3.2 \\
\hline$\frac{w^{+}}{w^{-}} ; \frac{T m 9 \text {-lex Ap } 65^{\text {att } P 40}}{\text { SSO0359(Dm12-splitGAL4) }} ; \frac{\text { lexAop-GFP } P^{11}, U A S-s y b: G F P^{1-10}}{S S 00359(D m 12 \text {-splitGAL4) }}$ & 3.2 \\
\hline$\frac{w^{+}}{w^{-}} ; \frac{T m 9-l e x A p 65^{a t t P 40}}{+} ; \frac{\text { lexAop-GFP } P^{11}, U A S-s y b:: G F P^{1-10}}{D m 20(V T 049111)-G A L 4}$ & 3.2 (n.s.) \\
\hline$\frac{w^{+}}{w^{-}} ; \frac{S S 02361(D m 4-s p l i t G A L 4)}{U A S-m C D 8:: G F P} ; \frac{S S 02361(D m 4-s p l i t G A L 4)}{+}$ & 3.2 \\
\hline$\frac{w^{+}}{w^{-}} ; \frac{S S 00359(D m 12-s p l i t G A L 4)}{U A S-m C D 8:: G F P} ; \frac{S S 00359(D m 12-s p l i t G A L 4)}{+}$ & 3.2 \\
\hline$\frac{w^{+}}{w^{-}} ; \frac{U A S-m C D 8:: G F P}{+} ; \frac{D m 20(V T 049111)-G A L 4}{+}$ & 3.2 \\
\hline$\frac{w^{+}}{w^{-}} ; \frac{S S 02361(D m 4-s p l i t G A L 4)}{C y O} ; \frac{S S 02361(D m 4 \text {-splitGAL4) }}{U A S \text {-DenMark,UAS-syt::eGFP }}$ & $3.3,3.4$ \\
\hline$\frac{w^{+}}{w^{-}} ; \frac{S S 00359(D m 12-\text { splitGAL4) }}{C y O} ; \frac{S S 00359(D m 12 \text {-split } G A L 4)}{U A S-D e n M a r k, U A S-\text { syt::eGFP }}$ & 3.3 \\
\hline$\frac{w^{+}}{w^{-}} ; \frac{S S 02361(D m 4-s p l i t G A L 4)}{+} ; \frac{S S 02361(D m 4-s p l i t G A L 4)}{U A S-s y t:: R F P}$ & 3.4 \\
\hline$\frac{w^{+}}{w^{-}} ; \frac{\text { SS02361(Dm4-splitGAL4) }}{+} ; \frac{\text { SS02361(Dm4-splitGAL4) }}{U A S-b r p^{\text {short }}:: m C h e r r y}$ & 3.4 \\
\hline 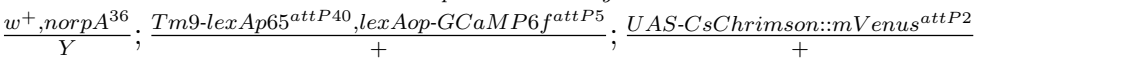 & 3.6 \\
\hline 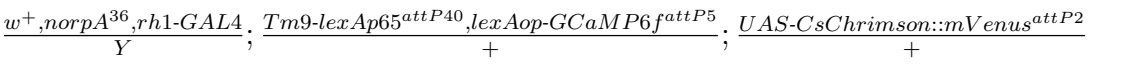 & $3.6,3.7$ \\
\hline 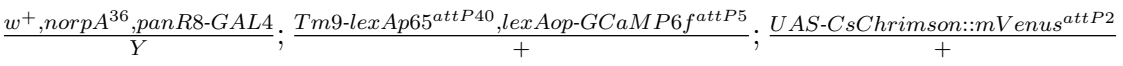 & 3.7 \\
\hline 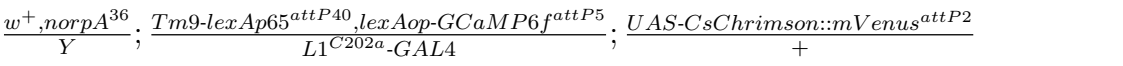 & $3.6,3.7$ \\
\hline 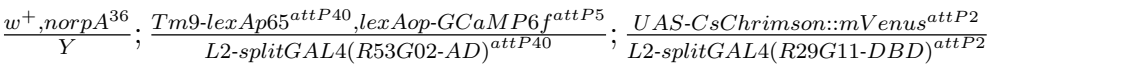 & 3.7 \\
\hline 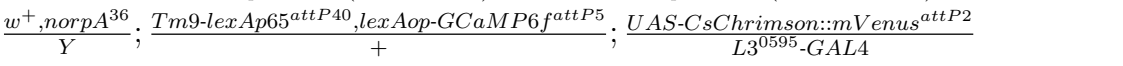 & $3.6,3.7$ \\
\hline 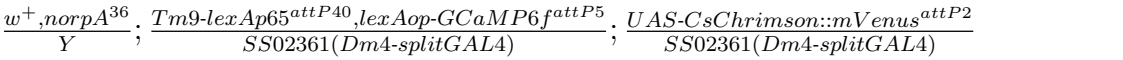 & 3.6 \\
\hline 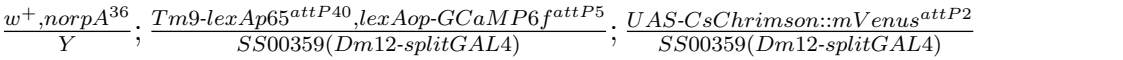 & $3.6,3.7$ \\
\hline 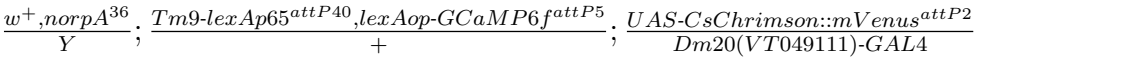 & $3.6,3.7$ \\
\hline 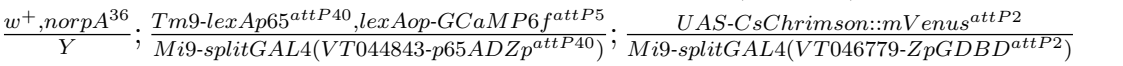 & 3.7 \\
\hline$\frac{w^{+}, \text {norp } A^{36}}{Y} ; \frac{\text { Tm9-lexAp } 65^{\text {att } P 40}, \text { lexAop-GCaMP } 6 f^{\text {att } P 5}}{+} ; \frac{U A S-C s C h r i m s o n:: m V e n u s^{a t t P 2}}{T m 1^{27 b}-G A L 4}$ & 3.7 \\
\hline 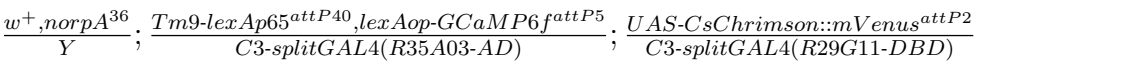 & 3.7 \\
\hline
\end{tabular}

n.s.: data not shown in figure 
Table 6.3: Genotypes of experimental lines used in sections 3.2 and 3.3.

\begin{tabular}{|c|c|}
\hline Genotype & Used in figs. \\
\hline$\frac{w^{+}}{w^{+}} ; \frac{T m 9-l e x A p 65^{a t t P 40}, l e x A o p-G C a M P 6 f^{a t t P 5}}{+} ; \frac{U A S-K i r 2.1:: e G F P}{+}$ & 3.8-17 \\
\hline$\frac{w^{+}}{w^{+}} ; \frac{T m 9-l e x A p 65^{a t t P 40}, l e x A o p-G C a M P 6 f^{a t t P 5}}{L 1^{C 202 a}-G A L 4} ; \frac{U A S-K i r 2.1:: e G F P}{+}$ & $3.8-12$ \\
\hline 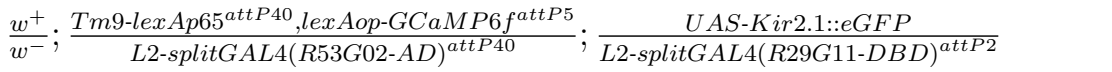 & $3.8-12$ \\
\hline 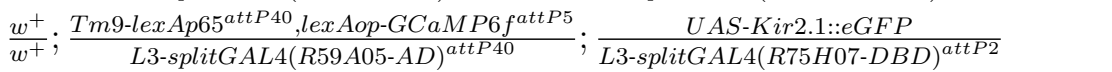 & $3.8-12$ \\
\hline 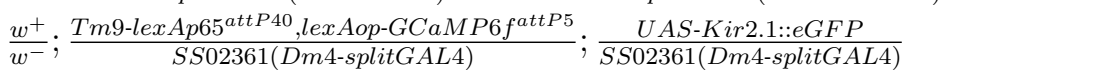 & 3.13-17 \\
\hline$\frac{w^{+}}{w^{-}} ; \frac{T m 9-l e x A p 65^{a t t P 40}, l e x A o p-G C a M P 6 f^{a t t P 5}}{+} ; \frac{U A S-K i r 2.1:: e G F P}{D m 9^{42 H 01}-G A L 4^{a t t P 2}}$ & $3.13-17$ \\
\hline 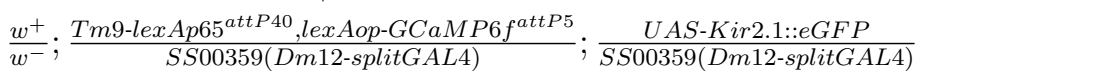 & $3.13-17$ \\
\hline$\frac{w^{+}}{w^{-}} ; \frac{T m 9-l e x A p 65^{a t t P 40}, \text { lexAop-GCaMP6f attP5 }}{+} ; \frac{U A S-K i r 2.1:: e G F P}{D m 20(V T 049111)-G A L 4}$ & $3.13-17$ \\
\hline$\frac{w^{+}}{w^{+}} ; \frac{T m 9-l e x A p 65^{a t t P 40}, \text { lexAop-GCaMP } 6 f^{a t t P 5}}{+} ; \frac{U A S \text {-shakB.RNAi(TRiP.HMC04895) })^{a t t P 2}}{+}$ & $3.18-21$ \\
\hline$\frac{w^{+}}{w^{+}} ; \frac{T m 9-l e x A p 65^{a t t P 40}, \text { lexAop-GCaMP } 6 f^{a t t P 5}}{+} ; \frac{U A S \text {-shakB.RNAi(TRiP.HMC04895) })^{a t t P 2}}{T m 9^{42 C 08}-G A L 4}$ & $3.18-21$ \\
\hline
\end{tabular}

Table 6.4: Genotypes of experimental lines used in chapter 4.

Genotype

$\frac{w^{+}}{w^{-}} ; \frac{S S 02361(D m 4-s p l i t G A L 4)}{U A S-G C a M P 6 f} ; \frac{S S 02361(D m 4-s p l i t G A L 4)}{+}$

$\frac{w^{+}}{w^{-}} ; \frac{S S 00359(D m 12-s p l i t G A L 4)}{U A S-G C a M P 6 f} ; \frac{S S 00359(D m 12-s p l i t G A L 4)}{+}$

$\frac{w^{+}}{w^{+}} ; \frac{T 4 / T 5^{R 59 E 08} \text {-lexA,lexAop-GCaMP6f } \text { attP5 }^{\text {at }}}{+} ; \frac{U A S-K i r 2.1:: e G F P}{+}$

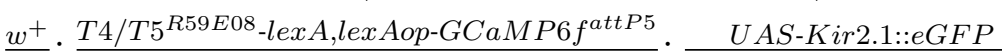

$\frac{w^{+}}{w^{-}} ; \frac{T 4 / T 5}{S S 02361(\text { Dm4-splitGAL4) }} ; \frac{U A S-K i r 2.1:: e G F P}{S S 02361(\text { Dm4-splitGAL4) }}$

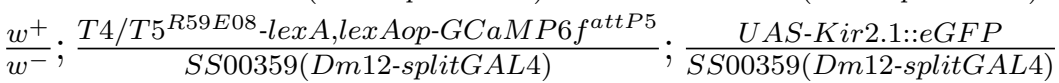

$\frac{w^{+}}{w^{-}}: \frac{T 4 / T 5^{R 59 E 08} \text {-lexA,lexAop-GCaMP6f attP5 }}{\text { a }} ; \frac{U A S-K i r 2.1:: e G F P}{D}$

$\frac{w^{-}}{+} ; \frac{T 4 / T 5 R 9 E}{D m 20(V T 049111)-G A L 4}$

$\frac{w^{+}}{w^{+}} ; \frac{T 4 / T 5^{R 59 E 08} \text {-lexA,lexAop-GCaMP6f } \text { attP5 }^{a t}}{+} ; \frac{U A S-K i r 2.1:: e G F P}{T m 9^{42 C 08-G A L 4}}$

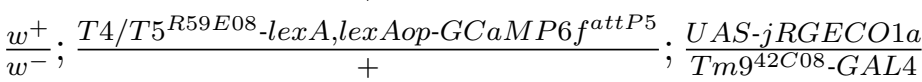

Used in figs.

$4.1,4.2$

$4.1,4.2$

4.3-12

4.4-12

4.4-12

4.4-12

4.4-12

4.13, 4.14 


\subsection{Fly immunostainings}

Flies were mounted, up to 6 flies were dissected at a time. They were lined up head up. With forceps rip off proboscis sequentially for each fly. Then in the mid-line of the anterior part of head rip from opening from proboscis removal up to the top of head (in between the eyes), also sequentially for each fly. Now we focus on one fly, we rip off the cuticle, especially from the eyes. This is done by inserting forceps (number 5) just below the eyes but on top of the brain, so the retina is removed but the rest of the brain starting by the lamina remains. The remains of retina (red spongy stuff) need to be cleaned of so it does not absorb the antibodies later on, and to reduce autofluorescence and occlusion of the rest of the optic lobe. This is critical step where lamina could be detached or damaged, if this did not already happen while removing the cuticle. Next the trachea can be removed, this is a white translucent looking membrane. After this, we can detach the brain from the body by carefully cutting below the head. Then, proceed to clean the trachea in the newly exposed brain portion. Brains were dissected in PFA $2 \%$ solution, deposited in the same ice cold PFA solution while the rest of dissections were going on.

Brains were left for about one hour in the PFA at room temperature, then washed three times in $0.3 \%$ PBT and blocked with normal goat serum (NGS) overnight at $4{ }^{\circ} \mathrm{C}$. NGS was replaced with the primary antibody solution and left for $48 \mathrm{~h}$ at $4^{\circ} \mathrm{C}$. Then again washed trice in PBT, each $5 \mathrm{~min}$. Secondary antibody was added and incubated overnight at $4{ }^{\circ} \mathrm{C}$ or $2 \mathrm{~h}$ at room temperature. Brains were washed trice again in PBT to lastly add embedding medium (Vectashield).

Antibody solutions were $10 \%$ in NGS. For GFP we used chicken antiGFP (Abcam, ab13970) at 1:2000, for mCherry and RPF we used rabbit anti-dsRed (Clontech, 632496) at 1:400. Secondary antibodies were Alexa Fluor 488 anti-chicken IgG (Dianova, 103545155) and Alexa Fluor 594 anti-rabbit IgG (Dianova, 111585003) both at 1:200.

We imaged the brains using a Zeiss LSM confocal microscope with 40x or $60 x$ oil objectives.

\subsection{Fly in vivo two-photon calcium imaging}

\section{Setup}

For in vivo two-photon calcium imaging we used a Bruker Investigator 2-photon microscope. GCaMP6f was excited using a Insight laser (Spec- 
traphysics) tuned at $920 \mathrm{~nm}$ for dual channel with jRGECO1a laser was tuned to $935 \mathrm{~nm}$ for Tm4 and Tm9 experiments, or $980 \mathrm{~nm}$ for T5 and Tm9 experiments. We used a Nikon 25X/1.1 objective. Zoom level was 8 to 10 for Tm and Dm neuron recordings but 6 for T4 and T5 recordings. Scanning rate was about $10 \mathrm{~Hz}$. The microscope was controlled by PrairieView software. Communication of stimulus computer and the microscope was performed via a NI USB-611 data acquisition card.

\section{Dissection}

We opened a optical window to the fly brain by removing cuticle in the back of the head and underlying fat and trachea. Dissections were performed in ice cold saline solution without calcium. Imaging solution was at room temperature and contained calcium. Saline solutions are same as used in (Silies et al., 2013).

\section{Visual stimulation}

Visual stimulation consisted of a DLP LightCrafter 4500 (Texas Instruments) controlled by custom-written software in C++ using OpenGL. Resolution was 912 x 1140 pixels and frame rate was $100 \mathrm{~Hz}$. Stimulus were projected on a squared screen of $8 \mathrm{~cm}$ sides which was placed so it covered $60^{\circ}$ of visual angle from the fly position under the objective.

\subsection{Optogenetic input mapping}

\section{Fly food with all-trans retinal (ATR)}

All procedures in this subsection were carried out in a dark room. A stock solution of $100 \mathrm{~mm}$ ATR in EtOH was prepared by diluting $25 \mathrm{mg}$ all-trans-retinal in $878.92 \mu \mathrm{l}$.

The food was supplemented with ATR solution to reach $1 \mathrm{mM}$ similarly as in (Hornstein, Pulver, and Griffith, 2009). Briefly, food was microwaved until it liquefied (usually for $30 \mathrm{~s}$ ). Food was let to cool down for about $30 \mathrm{~s}$. Vials contained $6 \mathrm{ml}$ to $10 \mathrm{ml}$. Once the food was cooler and still liquid, $60 \mu \mathrm{l}$ to $70 \mu \mathrm{l}$ of the stock ATR solution were pipetted into the food. Food was stirred promptly and vigorously to mix the ATR. After mixing, vial was placed into ice to solidify the supplemented food. After solidification, vials were cleaned from condensation and food in the walls. Flies were then placed into the food, and vials were wrapped into aluminum foil to block 
light. Vials with flies were put into the incubators at $25{ }^{\circ} \mathrm{C}$ for at least two days before experiments.

\section{Stimulation device}

LED from ThorLabs at $625 \mathrm{~nm}$. Light stimulation was delivered through the microscope objective.

\section{Stimulation protocols}

The stimuli consisted on a $625 \mathrm{~ms}$ long train of light pulses at $40 \mathrm{~Hz}$. Every pulse lasted $5 \mathrm{~ms}$ and had equal power $9.40 \mu \mathrm{W} \mathrm{mm}^{-2}$ to $29.40 \mu \mathrm{W} \mathrm{mm}^{-2}$. The first pulse started $10 \mathrm{~s}$ after recording start. Five trains were presented per recording, the trains were separated by $30 \mathrm{~s}$ from each other. Alternatively, a single pulse of $25 \mathrm{~ms}$, the shortest possible in our setup, was used with a power of $29.40 \mu \mathrm{W} \mathrm{mm}^{-2}$. The pulse started $10 \mathrm{~s}$ after recording start. Five pulses separated by $30 \mathrm{~s}$ from each other were presented per recording.

\subsection{Data processing}

\section{Image registration}

Image registration was performed for correcting motion artifacts and aligning multiple time series corresponding to different stimuli.

Most of the data was aligned by rigid registration, by translating images to maximize the cross-correlation of a given frame with a template. For two-channel images, the calculation of the position of the cross-correlation maximum was performed on the sum of the cross-correlations of the two channels. To obtain a template image the signal-to-noise ratio (SNR) was calculated for the first 300 frames of the time series (or the entire time series if it was shorter). The template was constructed by averaging the frames up to the start of the SNR plateau.

\section{Roi selection}

Regions of interest were selected manually for most of the data by drawing a polygon around the identified portions of neurites within an eye column from a maximum intensity projection of the aligned time series. The exception are T4T5 recordings in the lobula plate. There we used the 
correlation projection obtained by substituting each pixel by the average correlation of the time series with that of the neighboring pixels. Then a circle of the size matching local structure of the correlation image was used to manually select the ROI centers (about $2.75 \mu \mathrm{m}$ in diameter). Using this method, only responsive signals were selected, since low signals lead to lower correlations due to increased contribution of noise.

\section{Signal normalization}

Fluorescence signal was normalized relative to $F_{0}$ (eq. 6.1), the baseline fluorescence (mean signal of inter-stimulus background), per region-ofinterest, resulting in eq. 6.2. However, when the baseline signal is close to zero, the division results in large values. To account for low baselines we added the mean of the full time series to the denominator (eq. 6.3).

$$
\begin{aligned}
F_{0} & =\frac{1}{n} \sum_{i=1}^{n} F\left(t_{i}\right) ; \quad t_{i}=\{t \mid t \text { is inter-stimulus interval }\} \\
\frac{\Delta F(t)}{F_{0}} & =\frac{F(t)-F_{0}}{F_{0}} \\
\frac{\Delta F(t)}{F_{0}+} & =\frac{F(t)-F_{0}}{F_{0}+\frac{1}{T} \sum_{t=1}^{T} F(t)}
\end{aligned}
$$

\section{Signal quality metrics and data selection}

For receptive field measurements we defined two quality parameters: the (non-parametric) response quality index (eq. 6.4) and the (parametric) r-square of the Gaussian fits. Response quality index (RQI) was given by (Baden et al., 2016):

$$
\mathrm{RQI}=\frac{\operatorname{Var}\left[\langle\mathbf{F}\rangle_{r}\right]_{t}}{\left\langle\operatorname{Var}[\mathbf{F}]_{t}\right\rangle_{r}}
$$

where $\mathbf{F}$ is a $n_{t} \times n_{r}$ response matrix from $n_{t}$ time points and $n_{r}$ repetitions of the same stimulus, and $\operatorname{Var}[\cdot]_{x}$ and $\langle\cdot\rangle_{x}$ are the variance and mean across dimension $x$, respectively. If responses are identical across trials $\mathrm{RQI}=1$, and if responses are random over trials with fixed variance $\mathrm{RQI} \propto 1 / n_{r}$.

We visualized the relationship between the two and found linear relations for OFF RFs, because OFF RFs are largely captured by a single Gaussian. For ON RFs there is lower signal and larger variability, it is not 
trivial to decide what function will fit better, some appear like a difference of Gaussians centered around zero, others have their lowest response at zero and have two positive lobes around it. We decided to focus on the center, and fit a single Gaussian to it. The extent of this ON RF component is less or equal than the full ON RF. In the view of this simplification, the threshold for separating ROIs was higher for the nonparametric quality index and lower for the parametric goodness of fit. We set for 0.5 for response quality index and 0.2 for the r-square. Most quantifications required to pass all thresholds for all stimuli used to map RF of a given ROI. In cases where the ON RFs mapped were of lower SNR, the constraints were relaxed to require only good OFF RFs, and ON RF for either orientation. In cases when the effect of silencing could lead to loss of some RFproperties, the criteria were applied per stimulus. For measuring non-parametric properties we set the threshold only for the response quality index, since fits were bad for most ROIs in the given condition.

\subsection{ON-OFF full-field flashes analysis}

We quantified the magnitude and dynamics of responses to ON-OFFfullfield flashes. We calculated the mean of the response during the ON and the OFF flashes:

$$
\begin{aligned}
\left\langle\frac{\Delta F}{F_{0}}\right\rangle_{\mathrm{ON}} & =\frac{1}{T_{\mathrm{ON}}} \sum_{t \in \mathrm{ON}} \frac{\Delta F}{F_{0}} \\
\left\langle\frac{\Delta F}{F_{0}}\right\rangle_{\mathrm{OFF}} & =\frac{1}{T_{\mathrm{OFF}}} \sum_{t \in \mathrm{OFF}} \frac{\Delta F}{F_{0}}
\end{aligned}
$$

where $T_{\mathrm{ON}, \mathrm{OFF}}$ is the number of time points the given stimulus was presented.

Using the mean responses (eq. 6.5, 6.6) we defined the polarity index (Baden et al., 2016) as follows:

$$
\text { Polarity index }=\frac{\left\langle\frac{\Delta F}{F_{0}}\right\rangle_{\mathrm{ON}}-\left\langle\frac{\Delta F}{F_{0}}\right\rangle_{\mathrm{OFF}}}{\left|\left\langle\frac{\Delta F}{F_{0}}\right\rangle_{\mathrm{ON}}\right|+\left|\left\langle\frac{\Delta F}{F_{0}}\right\rangle_{\mathrm{OFF}}\right|}
$$

We quantified the response dynamics with the sustenance index defined as the ratio of mean response within the last $500 \mathrm{~ms}$ of the stimulus (ON or OFF flash) to the extreme response, which is the quantity that is larger in absolute magnitude between the minimum and maximum of the response. For transient responses that decay to zero towards the end of the stimulus 
the sustenance index equals zero, while the sustenance index equals one for sustained responses that remain constant during the stimulus or rise slowly to reach their maximum only at the end of the stimulus. To disambiguate between the last two situations we considered the time to extreme as an independent parameter that indicates whether the response peaks early or late. And to measure how fast the response evolves over time we used the half-rise time, which is the time point at which the response is equal to half of the extreme response. Both time to extreme and half-rise time are bounded between zero and the stimulus duration.

\subsection{Receptive field analysis}

\section{Flashing bars}

Response to the flashing bar at a given location was measured by the extreme of the time course.

This resulted in a tuning curve. The tuning curve was the fitted by a single Gaussian of the form

$$
f(x)=a \cdot e^{-\left(\frac{x-b}{c}\right)^{2}}
$$

The $a$ is the signal amplitude, $b$ is the receptive field center position and $c$ is related to the receptive field width. The width was given by the full width at half maximum (FWHM) as

$$
\mathrm{FHWM}=2 c \sqrt{\log (2)}
$$

To quantify the variability in receptive field sizes (FWHM) we used the standard deviation $\operatorname{std}_{\mathrm{FWHM}}$ and coefficient of variation $\mathrm{CV}_{\mathrm{FWHM}}$

$$
\mathrm{CV}_{\mathrm{FWHM}}=\frac{\operatorname{std}(\mathrm{FWHM})}{\operatorname{mean}(\mathrm{FWHM})},
$$

which is a normalized measure of variability relative to the mean.

\section{Noise bars}

The receptive field from noise bars was obtained through reverse correlation analysis. The response was modeled by a linear filter.

$$
F_{t}=k S_{t}
$$


$F_{t}$ is the response at time $t$ and $S_{t}$ is the stimulus history of a given specified length, preceding the response. We used ordinary least squares to obtain the filter from the linear equation. After the filter $k$ is obtained, we can do a rough analysis of the spatial and temporal properties separately. The maximum of the filter is identified, at that point a slice is taken in both the temporal and spatial dimensions to get the approximated spatial and temporal filters. The spatial filter extent is then quantified by the FWHM as for the static bars by fitting a single Gaussian (eq. 6.8).

\subsection{Moving edges}

The preference to $\mathrm{ON}$ or OFF edges was measured as the contrast selectivity index (CSI):

$$
\mathrm{CSI}=\frac{\left\langle\max R_{\mathrm{ON}, \theta}-\max R_{\mathrm{OFF}, \theta}\right\rangle_{\theta}}{\left\langle\max R_{\mathrm{ON}, \theta}+\max R_{\mathrm{OFF}, \theta}\right\rangle_{\theta}}
$$

where $\max R_{\mathrm{ON}, \theta}$ is the maximum response of the neuron to an $\mathrm{ON}$ edge, moving in the direction given by $\theta$, and $\langle\cdot\rangle_{\theta}$ indicates average over directions.

The preference to one direction of motion over the others was measured by the direction selectivity index (DSI):

$$
\begin{gathered}
\text { DSI }=\frac{\left|\sum_{\theta} R_{\theta} e^{i \theta}\right|}{\sum_{\theta}\left|R_{\theta}\right|} \\
\theta_{\text {pref }}=\arctan \frac{\Im R_{\text {mean }}}{\Re R_{\text {mean }}} \\
R_{\text {mean }}=\frac{\sum_{\theta} R_{\theta} e^{i \theta}}{\sum_{\theta} R_{\theta}}
\end{gathered}
$$




\section{Bibliography}

Adelson, E.H. and Bergen, J.R. (Feb. 1, 1985). "Spatiotemporal Energy Models for the Perception of Motion". Journal of the Optical Society of America A 2.2, 284.

Ahrens, M.B. et al. (May 2013). "Whole-Brain Functional Imaging at Cellular Resolution Using Light-Sheet Microscopy". Nature Methods 10.5, 413-420. pmid: 23524393.

Akin, O. et al. (2019). "Cell-Type-Specific Patterned Stimulus-Independent Neuronal Activity in the Drosophila Visual System during Synapse Formation". Neuron 101.5, 894-904.e5.

Allenby, G.M., Rossi, P.E., and McCulloch, R.E. (2005). "Hierarchical Bayes Models: A Practitioners Guide". SSRN Electronic Journal.

Ammer, G. et al. (Aug. 31, 2015). "Functional Specialization of Neural Input Elements to the Drosophila ON Motion Detector". Current Biology 25.17, 2247-2253.

Anderson, C. et al. (Dec. 18, 2017). "Natural Variation in Stochastic Photoreceptor Specification and Color Preference in Drosophila". eLife 6. pmid: 29251595.

Anstis, S.M. (Dec. 1, 1970). "Phi Movement as a Subtraction Process". Vision Research 10.12, 1411-IN5.

Arendt, D. et al. (Dec. 2016). "The Origin and Evolution of Cell Types". Nature Reviews Genetics 17.12, 744-757.

Arenz, A. et al. (Apr. 3, 2017). "The Temporal Tuning of the Drosophila Motion Detectors Is Determined by the Dynamics of Their Input Elements". Current Biology 27.7, 929-944.

Baden, T. et al. (Jan. 21, 2016). "The Functional Diversity of Retinal Ganglion Cells in the Mouse". Nature 529.7586, 345-350. pmid: 26735013.

Badwan, B.A. et al. (Aug. 2019). "Dynamic Nonlinearities Enable Direction Opponency in Drosophila Elementary Motion Detectors". Nature Neuroscience 22.8, 1318-1326. 
Bahl, A. et al. (June 2013). "Object Tracking in Motion-Blind Flies". Nature Neuroscience 16.6, 730-738.

Baines, R.A. et al. (Jan. 3, 2001). "Altered Electrical Properties in DrosophilaNeurons Developing without Synaptic Transmission". The Journal of Neuroscience 21.5, 1523-1531. pmid: 11222642.

Baker, M. (May 26, 2016). "1,500 Scientists Lift the Lid on Reproducibility". Nature News 533.7604, 452.

Barlow, H.B. and Levick, W.R. (June 1965). "The Mechanism of Directionally Selective Units in Rabbit's Retina.” The Journal of Physiology 178.3, 477-504. pmid: 5827909.

Behnia, R. et al. (Aug. 28, 2014). "Processing Properties of ON and OFF Pathways for Drosophila Motion Detection". Nature 512.7515, 427430.

Bellen, H.J. et al. (Sept. 1989). "P-Element-Mediated Enhancer Detection: A Versatile Method to Study Development in Drosophila". Genes \& Development 3.9, 1288-1300. pmid: 2558050.

Boiroju, D.N.K. and Reddy, D.M.K. (Dec. 13, 2012). "Bootstrap Method for Testing of Equality of Several Coefficients of Variation". International Journal of Advanced Mathematics and Statistics 1.1, pp. 1-6-6.

Boyden, E.S. et al. (Sept. 2005). "Millisecond-Timescale, Genetically Targeted Optical Control of Neural Activity". Nature Neuroscience 8.9, 1263-1268. pmid: 16116447.

"BRAIN 2025: A Scientific Vision" (n.d.) (), 146.

Braitenberg, V. (Mar. 1, 1967). "Patterns of Projection in the Visual System of the Fly. I. Retina-Lamina Projections". Experimental Brain Research 3.3, 271-298.

Brand, A.H. and Perrimon, N. (June 1993). "Targeted Gene Expression as a Means of Altering Cell Fates and Generating Dominant Phenotypes". Development (Cambridge, England) 118.2, 401-415. pmid: 8223268.

Briggman, K.L., Helmstaedter, M., and Denk, W. (Mar. 2011). "Wiring Specificity in the Direction-Selectivity Circuit of the Retina". Nature 471.7337, 183-188.

Briggman, K.L. and Bock, D.D. (Feb. 1, 2012). "Volume Electron Microscopy for Neuronal Circuit Reconstruction". Current Opinion in Neurobiology. Neurotechnology 22.1, 154-161.

Buchner, E., Buchner, S., and Bülthoff, I. (July 1, 1984). "Deoxyglucose Mapping of Nervous Activity Induced inDrosophila Brain by Visual Movement". Journal of Comparative Physiology A 155.4, 471-483.

Cadwell, C.R. et al. (Feb. 2016). "Electrophysiological, Transcriptomic and Morphologic Profiling of Single Neurons Using Patch-Seq". Nature Biotechnology 34.2, 199-203. 
Cai, D. et al. (May 5, 2013). "Improved Tools for the Brainbow Toolbox". Nature Methods 10.6, 540-547. pmid: 23817127.

Cajal, S. (1909). "Nota Sobre La Retina de La Mosca (M. Vomitoria L.)" Trab. Lab. Invest. Biol. Univ. Madrid 7, 217-257.

Cembrowski, M.S. and Menon, V. (June 1, 2018). "Continuous Variation within Cell Types of the Nervous System". Trends in Neurosciences 41.6, 337-348.

Cembrowski, M.S., Phillips, M.G., et al. (May 17, 2018). "Dissociable Structural and Functional Hippocampal Outputs via Distinct Subiculum Cell Classes". Cell 173.5, 1280-1292.e18.

Cembrowski, M.S. and Spruston, N. (Apr. 2019). "Heterogeneity within Classical Cell Types Is the Rule: Lessons from Hippocampal Pyramidal Neurons". Nature Reviews Neuroscience 20.4, 193.

Chen, T.-W. et al. (July 18, 2013). "Ultrasensitive Fluorescent Proteins for Imaging Neuronal Activity". Nature 499.7458, 295-300.

Chichilnisky, E.J. and Kalmar, R.S. (Jan. 4, 2002). "Functional Asymmetries in ON and OFF Ganglion Cells of Primate Retina". The Journal of Neuroscience 22.7, 2737-2747. pmid: 11923439.

Chou, W.H. et al. (Feb. 1999). "Patterning of the R7 and R8 Photoreceptor Cells of Drosophila: Evidence for Induced and Default Cell-Fate Specification". Development (Cambridge, England) 126.4, 607-616. pmid: 9895309.

Clark, D.A. et al. (June 23, 2011). "Defining the Computational Structure of the Motion Detector in Drosophila". Neuron 70.6, 1165-1177.

Coblis - Color Blindness Simulator - Colblindor (2019). URL: https : / / www . color-blindness . com/coblis-color-blindness-simulator/ (visited on 09/30/2019).

Cooper, E.A. and Norcia, A.M. (May 28, 2015). "Predicting Cortical Dark/Bright Asymmetries from Natural Image Statistics and Early Visual Transforms". PLoS Comput Biol 11.5, e1004268.

Dana, H. et al. (Mar. 24, 2016). "Sensitive Red Protein Calcium Indicators for Imaging Neural Activity". eLife 5. M. Häusser, ed., e12727.

Davie, K. et al. (Aug. 9, 2018). "A Single-Cell Transcriptome Atlas of the Aging Drosophila Brain”. Cell 174.4, 982-998.e20. pmid: 29909982.

Davis, F.P. et al. (Aug. 13, 2019). "A Genetic, Genomic, and Computational Resource for Exploring Neural Circuit Function". bioRxiv, 385476.

Denk, W. and Horstmann, H. (Oct. 19, 2004). "Serial Block-Face Scanning Electron Microscopy to Reconstruct Three-Dimensional Tissue Nanostructure". PLOS Biology 2.11, e329. 
Diamond, J.S. (Sept. 15, 2017). "Inhibitory Interneurons in the Retina: Types, Circuitry, and Function". Annual Review of Vision Science 3.1, $1-24$.

Dionne, H. et al. (May 1, 2018). "Genetic Reagents for Making Split-GAL4 Lines in Drosophila". Genetics 209.1, 31-35. pmid: 29535151.

Ecker, J.R. et al. (Nov. 1, 2017). "The BRAIN Initiative Cell Census Consortium: Lessons Learned toward Generating a Comprehensive Brain Cell Atlas". Neuron 96.3, 542-557. pmid: 29096072.

Farnham, A. et al. (Nov. 15, 2017). "Early Career Researchers Want Open Science". Genome Biology 18.1, 221. pmid: 29141654.

Feinberg, E.H. et al. (Feb. 7, 2008). "GFP Reconstitution Across Synaptic Partners (GRASP) Defines Cell Contacts and Synapses in Living Nervous Systems". Neuron 57.3, 353-363.

Feng, G. et al. (Oct. 1, 2000). "Imaging Neuronal Subsets in Transgenic Mice Expressing Multiple Spectral Variants of GFP". Neuron 28.1, 4151. pmid: 11086982.

Fischbach, K.-F. and Dittrich, A. (Dec. 1989). "The Optic Lobe of Drosophila Melanogaster. I. A Golgi Analysis of Wild-Type Structure". Cell and Tissue Research 258.3.

Fischer, J.A. et al. (Apr. 28, 1988). "GAL4 Activates Transcription in Drosophila". Nature 332.6167, 853-856. pmid: 3128741.

Fisher, Y.E., Leong, J.C.S., et al. (2015). "A Class of Visual Neurons with Wide-Field Properties Is Required for Local Motion Detection". Current Biology.

Fisher, Y.E., Silies, M., and Clandinin, T.R. (Oct. 2015). "Orientation Selectivity Sharpens Motion Detection in Drosophila". Neuron 88.2, 390402.

Flyem Tables (2017). URL: http : / / emanalysis . janelia . org/flyem _ tables.php (visited on 08/10/2017).

Franke, K. et al. (Feb. 2017). "Inhibition Decorrelates Visual Feature Representations in the Inner Retina". Nature 542.7642, 439-444.

Freeman, J. et al. (Oct. 30, 2015). "Mapping Nonlinear Receptive Field Structure in Primate Retina at Single Cone Resolution". eLife 4. pmid: 26517879.

Freifeld, L. et al. (June 19, 2013). "GABAergic Lateral Interactions Tune the Early Stages of Visual Processing in Drosophila”. Neuron 78.6, 10751089. pmid: 23791198.

Gohl, D.M. et al. (Mar. 2011). "A Versatile in Vivo System for Directed Dissection of Gene Expression Patterns". Nature Methods 8.3, 231-237. pmid: 21473015. 
Gollisch, T. and Meister, M. (Jan. 28, 2010). "Eye Smarter than Scientists Believed: Neural Computations in Circuits of the Retina". Neuron 65.2, 150-164. pmid: 20152123.

Good, I.J. (Feb. 1, 1980). "Some History of the Hierarchical Bayesian Methodology". Trabajos de Estadistica Y de Investigacion Operativa $31.1,489$.

Gouwens, N.W. et al. (July 2019). "Classification of Electrophysiological and Morphological Neuron Types in the Mouse Visual Cortex". Nature Neuroscience 22.7, 1182-1195.

Gruntman, E., Romani, S., and Reiser, M.B. (Feb. 2018). "Simple Integration of Fast Excitation and Offset, Delayed Inhibition Computes Directional Selectivity in Drosophila". Nature Neuroscience 21.2, 250 257. pmid: 29311742.

Haag, J., Arenz, A., et al. (Aug. 9, 2016). "Complementary Mechanisms Create Direction Selectivity in the Fly". eLife 5, e17421. pmid: 27502554.

Haag, J., Mishra, A., and Borst, A. (Aug. 22, 2017). "A Common Directional Tuning Mechanism of Drosophila Motion-Sensing Neurons in the ON and in the OFF Pathway". eLife 6, e29044.

Hadjieconomou, D. et al. (Mar. 2011). "Flybow: Genetic Multicolor Cell Labeling for Neural Circuit Analysis in Drosophila Melanogaster". Nature Methods 8.3, 260-266. pmid: 21297619.

Hampel, S. et al. (Mar. 2011). "Drosophila Brainbow: A RecombinaseBased Fluorescence Labeling Technique to Subdivide Neural Expression Patterns". Nature Methods 8.3, 253-259. pmid: 21297621.

Hassenstein, B. and Reichardt, W. (Sept. 1956). "Systemtheoretische Analyse Der Zeit-, Reihenfolgen- Und Vorzeichenauswertung Bei Der Bewegungsperzeption Des Rüsselkäfers Chlorophanus". Zeitschrift für Naturforschung B 11.9, 513-524.

Heisenberg, M. and Buchner, E. (Jan. 1, 1977). "The Rôle of Retinula Cell Types in Visual Behavior ofDrosophila Melanogaster". Journal of comparative physiology 117.2, 127-162.

Helmstaedter, M. et al. (Aug. 2013). "Connectomic Reconstruction of the Inner Plexiform Layer in the Mouse Retina". Nature 500.7461, 168174.

Hiesinger, P.R. and Hassan, B.A. (Sept. 2018). "The Evolution of Variability and Robustness in Neural Development". Trends in Neurosciences 41.9, 577-586.

Hobert, O., Glenwinkel, L., and White, J. (Nov. 21, 2016). "Revisiting Neuronal Cell Type Classification in Caenorhabditis Elegans". Current Biology 26.22, R1197-R1203. 
Hornstein, N.J., Pulver, S.R., and Griffith, L.C. (Mar. 16, 2009). "Channelrhodopsin2 Mediated Stimulation of Synaptic Potentials at Drosophila Neuromuscular Junctions". JoVE (Journal of Visualized Experiments) 25, e1133.

Horridge, G.A. and Meinertzhagen, I.A. (Dec. 1, 1970). "The Exact Neural Projection of the Visual Fields upon the First and Second Ganglia of the Insect Eye". Zeitschrift für vergleichende Physiologie 66.4, 369-378.

Hosoya, H. and Hyvärinen, A. (July 22, 2015). "A Hierarchical Statistical Model of Natural Images Explains Tuning Properties in V2". The Journal of Neuroscience: The Official Journal of the Society for Neuroscience 35.29, 10412-10428. pmid: 26203137.

Hughes, N.J. and Goodhill, G.J. (Jan. 2015). "Optimizing the Representation of Orientation Preference Maps in Visual Cortex". Neural Computation 27.1, 32-41.

Al-Hussain Bani Hani, S., Al-Haidari, M.H., and Saboba, M.M. (Sept. 20, 2007). "Neuronal Types in the Human Anterior Ventral Thalamic Nucleus: A Golgi Study". Cellular and Molecular Neurobiology 27.6, 745755.

Jenett, A. et al. (Oct. 25, 2012). "A GAL4-Driver Line Resource for Drosophila Neurobiology". Cell Reports 2.4, 991-1001. pmid: 23063364.

Joesch, M. et al. (Nov. 11, 2010). "ON and OFF Pathways in Drosophila Motion Vision". Nature 468.7321, 300-304.

Kale, A. et al. (Jan. 2019). "Hypothetical Outcome Plots Help Untrained Observers Judge Trends in Ambiguous Data". IEEE Transactions on Visualization and Computer Graphics 25.1, 892-902.

Karuppudurai, T. et al. (Feb. 5, 2014). "A Hard-Wired Glutamatergic Circuit Pools and Relays UV Signals to Mediate Spectral Preference in Drosophila". Neuron 81.3, 603-615. pmid: 24507194.

Keleş, M.F. and Frye, M.A. (Mar. 6, 2017). "Object-Detecting Neurons in Drosophila”. Current biology: CB 27.5, 680-687. pmid: 28190726.

Klapoetke, N.C. et al. (Mar. 2014). "Independent Optical Excitation of Distinct Neural Populations". Nature Methods 11.3, 338-346.

Konstantinides, N. et al. (July 26, 2018). "Phenotypic Convergence: Distinct Transcription Factors Regulate Common Terminal Features". Cell 174.3, 622-635.e13. pmid: 29909983.

Krakauer, J.W. et al. (Feb. 8, 2017). "Neuroscience Needs Behavior: Correcting a Reductionist Bias". Neuron 93.3, 480-490.

Krapp, H.G., Hengstenberg, B., and Hengstenberg, R. (Apr. 1998). "Dendritic Structure and Receptive-Field Organization of Optic Flow Processing Interneurons in the Fly". Journal of Neurophysiology 79.4, 19021917. pmid: 9535957. 
Leong, J.C.S. et al. (Mar. 8, 2016). "Direction Selectivity in Drosophila Emerges from Preferred-Direction Enhancement and Null-Direction Suppression". The Journal of Neuroscience 36.31, 8078-8092. pmid: 27488629.

Leonhardt, A., Ammer, G., et al. (May 2016). "Asymmetry of Drosophila ON and OFF Motion Detectors Enhances Real-World Velocity Estimation". Nature Neuroscience 19.5, 706-715.

Leonhardt, A., Meier, M., et al. (2017). "Neural Mechanisms Underlying Sensitivity to Reverse-Phi Motion in the Fly". PloS One 12.12, e0189019. pmid: 29261684.

Liu, J.K. et al. (July 26, 2017). "Inference of Neuronal Functional Circuitry with Spike-Triggered Non-Negative Matrix Factorization". Nature Communications 8.1, 149. pmid: 28747662.

Livet, J. et al. (Nov. 2007). "Transgenic Strategies for Combinatorial Expression of Fluorescent Proteins in the Nervous System". Nature 450.7166, $56-62$.

Llinás, R.R. (Jan. 2003). "The Contribution of Santiago Ramon y Cajal to Functional Neuroscience". Nature Reviews Neuroscience 4.1, 77-80.

Luo, L., Callaway, E.M., and Svoboda, K. (Mar. 13, 2008). "Genetic Dissection of Neural Circuits". Neuron 57.5, 634-660. pmid: 18341986.

- (Apr. 18, 2018). "Genetic Dissection of Neural Circuits: A Decade of Progress". Neuron 98.2, 256-281. pmid: 29673479.

Macpherson, L.J. et al. (Dec. 4, 2015). "Dynamic Labelling of Neural Connections in Multiple Colours by Trans-Synaptic Fluorescence Complementation". Nature Communications 6.1, 1-9.

Maheswaranathan, N. et al. (Aug. 2018). "Inferring Hidden Structure in Multilayered Neural Circuits". PLoS computational biology 14.8, e1006291. pmid: 30138312.

Maisak, M.S. et al. (Aug. 2013). "A Directional Tuning Map of Drosophila Elementary Motion Detectors". Nature 500.7461, 212-216.

Markram, H. et al. (Oct. 8, 2015). "Reconstruction and Simulation of Neocortical Microcircuitry". Cell 163.2, 456-492. pmid: 26451489.

Masland, R.H. (July 13, 2004). "Neuronal Cell Types". Current Biology 14.13, R497-R500.

Masland, R.H. (Oct. 18, 2012). "The Neuronal Organization of the Retina". Neuron 76.2, 266-280.

McKay, R.R. et al. (Feb. 6, 1995). "Phospholipase C Rescues Visual Defect in norpA Mutant of Drosophila Melanogaster". Journal of Biological Chemistry 270.22, 13271-13276. pmid: 7768926.

McKiernan, E.C. et al. (July 7, 2016). "How Open Science Helps Researchers Succeed". eLife 5. pmid: 27387362. 
Meier, M. and Borst, A. (May 2019). "Extreme Compartmentalization in a Drosophila Amacrine Cell". Current Biology 29.9, 1545-1550.e2.

Meinertzhagen, I.A. and O’Neil, S.D. (Mar. 8, 1991). "Synaptic Organization of Columnar Elements in the Lamina of the Wild Type in Drosophila Melanogaster". The Journal of Comparative Neurology 305.2, 232-263. pmid: 1902848.

Montell, C. et al. (May 1, 1987). "A Second Opsin Gene Expressed in the Ultraviolet-Sensitive R7 Photoreceptor Cells of Drosophila Melanogaster". Journal of Neuroscience 7.5, 1558-1566. pmid: 2952772.

Moriyoshi, K. et al. (Feb. 1996). "Labeling Neural Cells Using Adenoviral Gene Transfer of Membrane-Targeted GFP". Neuron 16.2, 255-260.

Munafò, M.R., Hollands, G.J., and Marteau, T.M. (Oct. 15, 2018). "Open Science Prevents Mindless Science". BMJ 363, k4309. pmid: 30322848.

Namboodiri, V.M.K., Rodriguez-Romaguera, J., and Stuber, G.D. (Oct. 10, 2016). "The Habenula". Current Biology 26.19, R873-R877.

Nern, A., Pfeiffer, B.D., and Rubin, G.M. (June 2, 2015). "Optimized Tools for Multicolor Stochastic Labeling Reveal Diverse Stereotyped Cell Arrangements in the Fly Visual System". Proceedings of the National Academy of Sciences 112.22, E2967-E2976.

Nguyen, J.P. et al. (Feb. 23, 2016). "Whole-Brain Calcium Imaging with Cellular Resolution in Freely Behaving Caenorhabditis Elegans". Proceedings of the National Academy of Sciences 113.8, E1074-E1081.

Nicolaï, L.J.J. et al. (Nov. 23, 2010). "Genetically Encoded Dendritic Marker Sheds Light on Neuronal Connectivity in Drosophila". Proceedings of the National Academy of Sciences 107.47, 20553-20558. pmid: 21059961.

Niven, J.E. and Laughlin, S.B. (June 2008). "Energy Limitation as a Selective Pressure on the Evolution of Sensory Systems". The Journal of Experimental Biology 211 (Pt 11), 1792-1804. pmid: 18490395.

O'Tousa, J.E. et al. (Apr. 1, 1985). "The Drosophila ninaE Gene Encodes an Opsin". Cell 40.4, 839-850. pmid: 2985266.

Ostroy, S.E., Wilson, M., and Pak, W.L. (Aug. 5, 1974). "Drosophila Rhodopsin: Photochemistry, Extraction and Differences in the Norp AP12 Phototransduction Mutant". Biochemical and Biophysical Research Communications 59.3, 960-966.

Packer, A.M. et al. (Feb. 2015). "Simultaneous All-Optical Manipulation and Recording of Neural Circuit Activity with Cellular Resolution in Vivo". Nature Methods 12.2, 140-146. pmid: 25532138.

Pandey, S. et al. (Apr. 2, 2018). "Comprehensive Identification and Spatial Mapping of Habenular Neuronal Types Using Single-Cell RNA-Seq". Current Biology 28.7, 1052-1065.e7. pmid: 29576475. 
Pfeiffer, B.D. et al. (Oct. 1, 2010). "Refinement of Tools for Targeted Gene Expression in Drosophila". Genetics 186.2, 735-755. pmid: 20697123. Phelan, P. and Starich, T.A. (May 2001). "Innexins Get into the Gap". BioEssays: News and Reviews in Molecular, Cellular and Developmental Biology 23.5, 388-396. pmid: 11340620.

Phelan, P., Stebbings, L.A., et al. (Jan. 8, 1998). "Drosophila ShakingB Protein Forms Gap Junctions in Paired Xenopus Oocytes". Nature 391.6663, 181-184. pmid: 9428764.

Poulin, J.-F. et al. (Sept. 2016). "Disentangling Neural Cell Diversity Using Single-Cell Transcriptomics". Nature Neuroscience 19.9, 1131-1141.

Ramon y Cajal, S. and Sánchez, S. (1915). "Contribución al Conocimiento de Los Centros Nerviosos de Los Insectos, Pt 1, Retina y Centros Opticos".

Ramos-Traslosheros, G., Henning, M., and Silies, M. (May 25, 2018). "Motion Detection: Cells, Circuits and Algorithms". Neuroforum 24, A61A72.

Reiff, D.F. et al. (Aug. 2010). "Visualizing Retinotopic Half-Wave Rectified Input to the Motion Detection Circuitry of Drosophila". Nature Neuroscience 13.8, 973-978.

Ribeiro, I.M.A. et al. (July 26, 2018). "Visual Projection Neurons Mediating Directed Courtship in Drosophila". Cell 174.3, 607-621.e18. pmid: 30033367.

Rister, J. et al. (Oct. 4, 2007). "Dissection of the Peripheral Motion Channel in the Visual System of Drosophila Melanogaster". Neuron 56.1, 155170.

Rivera-Alba, M. et al. (Dec. 6, 2011). "Wiring Economy and Volume Exclusion Determine Neuronal Placement in the Drosophila Brain". Current biology: CB 21.23, 2000-2005. pmid: 22119527.

Salazar-Gatzimas, E., Agrochao, M., et al. (Dec. 3, 2018). "The Neuronal Basis of an Illusory Motion Percept Is Explained by Decorrelation of Parallel Motion Pathways". Current Biology 28.23, 3748-3762.e8. pmid: 30471993.

Salazar-Gatzimas, E., Chen, J., et al. (Oct. 5, 2016). "Direct Measurement of Correlation Responses in Drosophila Elementary Motion Detectors Reveals Fast Timescale Tuning". Neuron 92.1, 227-239.

Salcedo, E. et al. (Dec. 15, 1999). "Blue- and Green-Absorbing Visual Pigments of Drosophila: Ectopic Expression and Physiological Characterization of the R8 Photoreceptor Cell-Specific Rh5 and Rh6 Rhodopsins". The Journal of Neuroscience: The Official Journal of the Society for Neuroscience 19.24, 10716-10726. pmid: 10594055. 
Sanes, J.R. and Masland, R.H. (2015). "The Types of Retinal Ganglion Cells: Current Status and Implications for Neuronal Classification". Annual Review of Neuroscience 38.1, null. pmid: 25897874.

Schnaitmann, C. et al. (Jan. 11, 2018). "Color Processing in the Early Visual System of Drosophila". Cell 172.1, 318-330.e18. pmid: 29328919.

Serbe, E. et al. (Feb. 2016). "Comprehensive Characterization of the Major Presynaptic Elements to the Drosophila OFF Motion Detector". Neuron. Seung, H.S. and Sümbül, U. (Sept. 17, 2014). "Neuronal Cell Types and Connectivity: Lessons from the Retina". Neuron 83.6, 1262-1272.

El-Shamayleh, Y., Ni, A.M., and Horwitz, G.D. (Jan. 7, 2016). "Strategies for Targeting Primate Neural Circuits with Viral Vectors". Journal of Neurophysiology 116.1, 122-134. pmid: 27052579.

Shekhar, K. et al. (Aug. 25, 2016). "Comprehensive Classification of Retinal Bipolar Neurons by Single-Cell Transcriptomics". Cell 166.5, 13081323.e30. pmid: 27565351.

Shi, Q. et al. (June 18, 2019). "Functional Characterization of Retinal Ganglion Cells Using Tailored Nonlinear Modeling". Scientific Reports 9.1, 8713. pmid: 31213620.

Shinomiya, K., Huang, G., et al. (Jan. 9, 2019). "Comparisons between the ON- and OFF-Edge Motion Pathways in the Drosophila Brain". eLife 8. A. Borst and K. VijayRaghavan, ed., e40025.

Shinomiya, K., Karuppudurai, T., et al. (May 19, 2014). "Candidate Neural Substrates for Off-Edge Motion Detection in Drosophila". Current Biology 24.10, 1062-1070.

Silies, M. et al. (July 10, 2013). "Modular Use of Peripheral Input Channels Tunes Motion-Detecting Circuitry". Neuron 79.1, 111-127.

Srivastava, U., Maurya, R., and Chand, P. (Apr. 1, 2009). "Cyto-Architecture and Neuronal Types of the Dorsomedial Cerebral Cortex of the Common Indian Wall Lizard, Hemidactylus Flaviviridis". Archives italiennes de biologie 147, 21-35.

Strother, J.A. et al. (Apr. 5, 2017). "The Emergence of Directional Selectivity in the Visual Motion Pathway of Drosophila". Neuron 94.1, 168182.e10.

Struhl, G. and Basler, K. (Feb. 26, 1993). "Organizing Activity of Wingless Protein in Drosophila". Cell 72.4, 527-540. pmid: 8440019.

Takemura, S.-y., Bharioke, A., et al. (Aug. 8, 2013). "A Visual Motion Detection Circuit Suggested by Drosophila Connectomics". Nature 500.7461, $175-181$.

Takemura, S.-y., Nern, A., et al. (Apr. 22, 2017). "The Comprehensive Connectome of a Neural Substrate for 'ON' Motion Detection in Drosophila'. eLife 6. A. Borst, ed., e24394. 
Takemura, S.-y., Xu, C.S., et al. (Nov. 3, 2015). "Synaptic Circuits and Their Variations within Different Columns in the Visual System of Drosophila". Proceedings of the National Academy of Sciences 112.44, 13711-13716. pmid: 26483464.

Talay, M. et al. (Oct. 26, 2017). "Transsynaptic Mapping of Second-Order Taste Neurons in Flies by Trans-Tango". Neuron.

Tan, L. et al. (Dec. 2015). "Ig Superfamily Ligand and Receptor Pairs Expressed in Synaptic Partners in Drosophila". Cell 163.7, 1756-1769.

Tasic, B., Menon, V., et al. (Feb. 2016). "Adult Mouse Cortical Cell Taxonomy Revealed by Single Cell Transcriptomics". Nature Neuroscience 19.2, 335-346.

Tasic, B., Yao, Z., et al. (Nov. 2018). "Shared and Distinct Transcriptomic Cell Types across Neocortical Areas". Nature 563.7729, 72-78.

The Internet Classics Archive — On the Sacred Disease by Hippocrates (2019). URL: http://classics.mit.edu/Hippocrates/sacred.html (visited on 09/21/2019).

The Petilla Interneuron Nomenclature Group (PING) (July 2008). "Petilla Terminology: Nomenclature of Features of GABAergic Interneurons of the Cerebral Cortex". Nature Reviews Neuroscience 9.7, 557-568.

Tirian, L. and Dickson, B.J. (Oct. 5, 2017). "The VT GAL4, LexA, and SplitGAL4 Driver Line Collections for Targeted Expression in the Drosophila Nervous System". bioRxiv, 198648.

Tobin, W.F., Wilson, R.I., and Lee, W.-C.A. (May 22, 2017). "Wiring Variations That Enable and Constrain Neural Computation in a Sensory Microcircuit". eLife 6. L. Luo, ed., e24838.

Turner, M.H. et al. (Jan. 2019). "Stimulus- and Goal-Oriented Frameworks for Understanding Natural Vision". Nature Neuroscience 22.1, 15.

Tuthill, J.C. et al. (July 10, 2013). "Contributions of the 12 Neuron Classes in the Fly Lamina to Motion Vision". Neuron 79.1, 128-140. pmid: 23849200.

Van Santen, J.P.H. and Sperling, G. (Feb. 1, 1985). "Elaborated Reichardt Detectors". Journal of the Optical Society of America A 2.2, 300.

Wardill, T.J. et al. (May 18, 2012). "Multiple Spectral Inputs Improve Motion Discrimination in the Drosophila Visual System". Science (New York, N.Y.) 336.6083, 925-931. pmid: 22605779.

Wässle, H. (Oct. 2004). "Parallel Processing in the Mammalian Retina". Nature Reviews Neuroscience 5.10, 747-757.

White, J.G. et al. (Nov. 12, 1986). "The Structure of the Nervous System of the Nematode Caenorhabditis Elegans". Philosophical Transactions of the Royal Society of London. B, Biological Sciences 314.1165, 1-340. 
Wienecke, C.F.R., Leong, J.C.S., and Clandinin, T.R. (Aug. 22, 2018). "Linear Summation Underlies Direction Selectivity in Drosophila". Neuron 99.4, 680-688.e4. pmid: 30057202.

Wong, A.M., Wang, J.W., and Axel, R. (Apr. 19, 2002). "Spatial Representation of the Glomerular Map in the Drosophila Protocerebrum". Cell 109.2, 229-241. pmid: 12007409.

Wu, M. et al. (Dec. 28, 2016). "Visual Projection Neurons in the Drosophila Lobula Link Feature Detection to Distinct Behavioral Programs". eLife 5. K. Scott, ed., e21022.

Yamaguchi, S. et al. (Mar. 25, 2008). "Motion Vision Is Independent of Color in Drosophila". Proceedings of the National Academy of Sciences of the United States of America 105.12, 4910-4915. pmid: 18353989.

Yamins, D.L.K. and DiCarlo, J.J. (Mar. 2016). "Using Goal-Driven Deep Learning Models to Understand Sensory Cortex". Nature Neuroscience 19.3, 356-365. pmid: 26906502.

Yang, H.H. and Clandinin, T.R. (Sept. 15, 2018). "Elementary Motion Detection in Drosophila : Algorithms and Mechanisms". Annual Review of Vision Science 4.1, 143-163.

Yang, H.H., St-Pierre, F., et al. (June 30, 2016). "Subcellular Imaging of Voltage and Calcium Signals Reveals Neural Processing In Vivo". Cell 166.1, 245-257. pmid: 27264607.

Yang, W. and Yuste, R. (Apr. 2017). "In Vivo Imaging of Neural Activity". Nature Methods 14.4, 349-359.

Zaqout, S.I. et al. (2012). "A Golgi Study of the Camel Cuneate Nucleus". The Anatomical Record 295.12, 2191-2204.

Zeisel, A. et al. (Mar. 6, 2015). "Cell Types in the Mouse Cortex and Hippocampus Revealed by Single-Cell RNA-Seq". Science 347.6226, 1138-1142. pmid: 25700174.

Zeng, H. and Sanes, J.R. (Sept. 2017). "Neuronal Cell-Type Classification: Challenges, Opportunities and the Path Forward". Nature Reviews Neuroscience 18.9, 530-546.

Zhang, Y.Q., Rodesch, C.K., and Broadie, K. (2002). "Living Synaptic Vesicle Marker: Synaptotagmin-GFP". genesis 34.1-2, 142-145.

Zheng, Z. et al. (July 26, 2018). "A Complete Electron Microscopy Volume of the Brain of Adult Drosophila Melanogaster". Cell 174.3, 730743.e22. pmid: 30033368.

Zuker, C.S., Montell, C., et al. (May 1, 1987). "A Rhodopsin Gene Expressed in Photoreceptor Cell R7 of the Drosophila Eye: Homologies with Other Signal-Transducing Molecules". Journal of Neuroscience 7.5, 15501557. pmid: 2437266. 
Zuker, C.S., Cowman, A.F., and Rubin, G.M. (Apr. 1, 1985). "Isolation and Structure of a Rhodopsin Gene from D. Melanogaster". Cell 40.4, 851-858. pmid: 2580638. 


\section{Acknowledgments}

I would like to thank my supervisor Prof. Marion Silies for the opportunity to research in her lab. I am grateful for her patience, encouragement and advice in my transition to fly experiments. I would also thank my committee members, Prof. Tim Gollisch and Prof. Fred Wolf for their feedback. My gratitude goes also to the neuroscience coordination office for their help since the arrival to Goettingen (Sandra, Michael, Jonas, and Franziska). I am happy to have great lab mates (Madhura, Katja, Burak, Sebastian, Miriam, Juan) and technician (Christine) and to have shared fun during lunch and the occasional game nights. I also enjoyed the coffee breaks with the regulars (Diego, Sindhu) with whom I had many discussions, including my office mate (Madhura). Even more I received the support from my friends in Goettingen, including past classmates and flatmates, and especially my partner (Özge) that accompanied me throughout the PhD. Finally, I am indebted to my family for their supportive freedom to pursue my scientific goals since my childhood. 


\section{Declaration}

Herewith I declare, that I prepared the doctoral dissertation Receptive field organization of motion computation in the fly: a study of cell types and their variability on my own and with no other sources and aids than quoted.

Luis Giordano Ramos Traslosheros Lopez

Göttingen, October 2019 